\title{
AVALIAÇÃO E APLICAÇÃO DE MODELOS DE ESTIMATIVA DE PRODUÇÃO DE CANA-DE-AÇÚCAR (Saccharum spp) BASEADOS EM PARÂMETROS DO SOLO E DO CLIMA
}

EDSON ROBERTO TERAMOTO

Tese apresentada àEscola Superior de Agricultura "Luiz de Queiroz", Universidade de São Paulo, para obtenção do título de Doutor em Agronomia, Área de Concentração: Fitotecnia.

PIRACICABA

Estado de São Paulo - Brasil

Janeiro - 2003 


\section{AVALIAÇÃO E APLICAÇÃO DE MODELOS DE ESTIMATIVA DE PRODUÇÃO DE CANA-DE-AÇÚCAR (Saccharum spp) BASEADOS EM PARÂMETROS DO SOLO E DO CLIMA}

EDSON ROBERTO TERAMOTO

Engenheiro Agrônomo

Orientador: Prof. Dr. Marcos Silveira Bernardes

Tese apresentada àEscola Superior de Agricultura "Luiz de Queiroz", Universidade de São Paulo, para obtenção do título de Doutor em Agronomia, Área de Concentração: Fitotecnia.

\section{PIRACICABA}

Estado de São Paulo - Brasil

Janeiro - 2003 


\section{Dados Internacionais de Catalogação na Publicação (CIP) DIVISÃo DE BIBLIOTECA E DOCUMENTAÇÃO - ESALQ/USP}

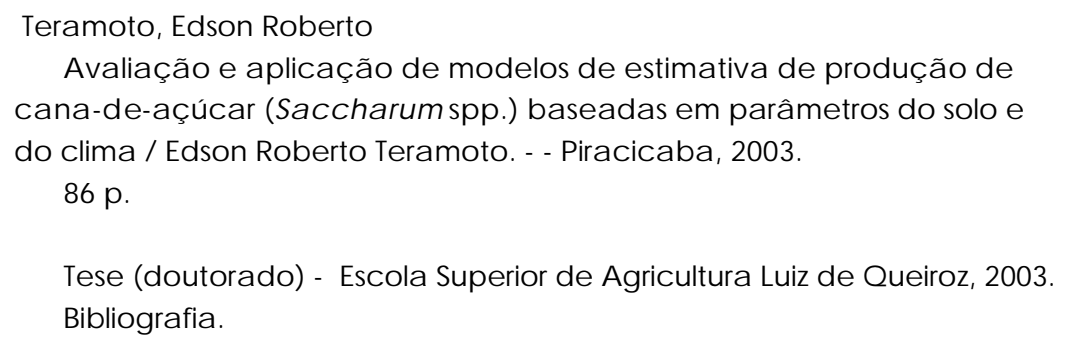

Tese (doutorado) - Escola Superior de Agricultura Luiz de Queiroz, 2003. Bibliografia.

1. Aptidão agrícola 2. Balanço hídrico 3. Cana-de-açúcar 4. $\begin{array}{lll}\text { Eva potranspiração } & \text { 5. Simulação 6. Terras I. Titulo }\end{array}$

CDD 633.61

\section{"Permitida a cópia total ou parcial deste documento, desde que citada a fonte - $O$ autor"}




\section{DEDICO}

a meus pais,

Nanami e Yuji

\section{OfEREÇO}

a minha Família,

Lia, Bruno e Gabriela 


\section{AGRADECIMENTOS}

À Escola Superior de Agricultura "Luiz de Queiroz" - Universidade de São Paulo e Departamento de Produção Vegetal pela oportunidade de realizar o curso.

Ao amigo Prof. Dr. Marcos Silveira Bernardes, pela orientação, apoio, ensinamentos de vida e compartilhamento de sua experiência que æ̀s vezes não enxergamos.

Ao amigo Prof. Dr. Gerd Sparovek, pela orientação, paciência, apoio incondicional e valiosos conselhos.

Ao Grupo COSAN - Usina Costa Pinto pelos dados que possibilitaram a realização deste trabalho.

Ao Engenheiro Agrônomo Helder Basaglia Zotelli, pela colaboração no fornecimento dos dados e no esclarecimento das dúvidas.

Ao Engenheiro Agrônomo Fabio R. Marins pela força nos cálculos de balanço hídrico.

Ao Engenheiro Agrônomo Marcelo C. Alves pelas análises estatísticas.

Ao Engenheiro Agrônomo Claudimir Penatti - Copersucar pelos trabalhos técnicos fornecidos.

Aos Professores Dr. Paulo Sentelhas, Dr. Nilson Villa Nova, Dr. Godofredo C. Vitti, Dr. Luiz Roberto Angelocci pelas sessões de tira-dúvidas.

Às Sras Edna, Inês, Lú e a grande Élide pela guarda de nossos anjos (Bruno e Gábi) e pelo porto seguro nos momentos complicados, muito obrigado.

Ao Prof. Dr. Gerd e ao acadêmico Beto (Alberto G. de O. P. Barreto) pela paciência e complicados comandos que geraram os mapas no SIG.

Aos amigos Vedete (Cirão) pelas revisões, Leila, Pakinha (Luis Fernando), Aureny, Edson Moraes, Ananias, Ilze que fizeram este trabalho um pouco mais leve.

À minha família, pela paciência e compreensão, obrigado pelo amor e carinho...

Enfim..., a todos que colaboraram e enviaram boas energias para este trabalho! 


\section{SUMÁRIO}

Página

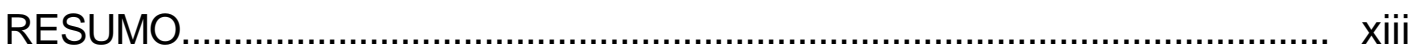

SUMMARY

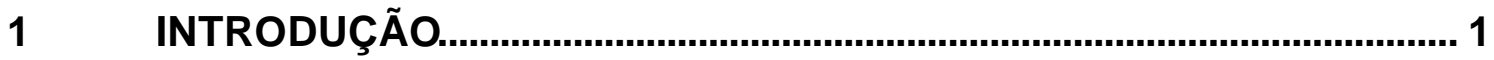

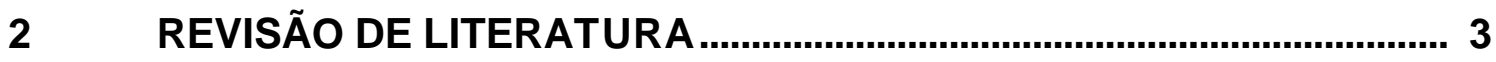

2.1 Importância e panorama atual da cana-de-açúcar no Brasil:................. 3

2.2 Aspectos climáticos para a cultura da cana -de-açúcar ........................... 4

2.2.1 Consumo de água pela cana-de-açúcar ................................................... 6

2.2.2 Balanço hídrico e evapotranspiração...................................................... 7

2.3 Relações entre solos e produção da cana-de-açúcar............................ 10

2.4 Modelos e simulações ............................................................................. 11

2.4.1 Modelagem em sistemas agrícolas ..................................................... 13

2.4.2 Modelos em cana-de-açúcar ............................................................ 16

2.5 Avaliação de terras, suporte àdecisão e planejamento regional ......... 17

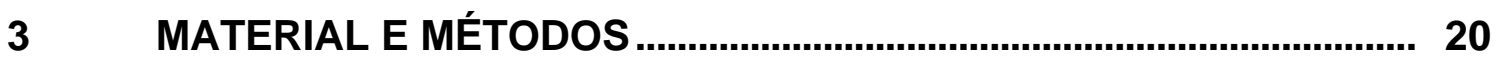

3.1 Localização das áreas com cana-de-açúcar ........................................ 20

3.2 Dados de produtividade e de análise de terra....................................... 20

3.3 Dados Climáticos .................................................................................. 21

3.4 Modelo para estimativa da produtividade relativa baseado em parâmetros do solo ......................................................... 21

3.4.1 Base teórica ..................................................................................... 22

3.4.2 Parâmetros utilizados e entrada de dados............................................. 23

3.4.3 Testes do modelo para estimativa da produtividade

3.4.4 Aplicação do modelo em escala regional ................................................ 26

3.5 Modelo FAO .................................................................................. 27

3.5.1 Produtividade Potencial - Método da Zona Agroecológica ................... 27

3.5.2 Produtividade realizável................................................................... 29

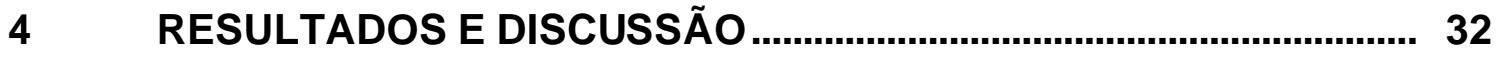

4.1 Testes do modelo em diferentes escalas ............................................. 32

4.1.1 Teste com dados experimentais ........................................................... 32 
4.1.2 Teste com dados de campo da Usina Santa Rita ................................ 34

4.1.3 Teste com dados de campo da Usina Costa Pinto ............................... 35

4.1.4 Causas da variação dos dados de produtividade obtidos ..................... 36

4.2 Potencial produtivo teórico das classes de solos avaliada através da produtividade medida da área de estudo.

4.3 Potencial produtivo teórico das classes de solos avaliada através do modelo para estimativa da produtividade relativa baseado em parâmetros do solo

4.4 Fatores que influenciaram a produtividade da cana-de-açúcar obtidos através de análise de regressão linear múltipla com seleção de variáveis pelo método stepwise

4.5 Aplicação prática do modelo para estimativa da produtividade relativa com base em parâmetros do solo

4.5.1 Distância das usinas em relação æ̀̀ áreas com cana-de-açúcar e às áreas do município

4.5.2 Ocorrência das áreas com cana-de-açúcar e das áreas do município em relação aos solos e seu potencial produtivo

4.5.3 Declividade das áreas ocupadas com cana-de-açúcar em relação às usinas e ao município

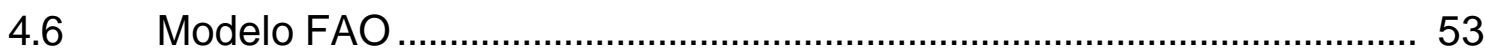

4.6.1 Produtividade realizável................................................................... 55

5 CONCLUSÕES 59 


\title{
AVALIAÇÃO E APLICAÇÃO DE MODELOS DE ESTIMATIVA DE PRODUÇÃO DE CANA-DE-AÇÚCAR (Saccharum spp) BASEADOS EM PARÂMETROS DO SOLO E DO CLIMA
}

\author{
Autor: EDSON ROBERTO TERAMOTO \\ Orientador: Prof. Dr. MARCOS SILVEIRA BERNARDES
}

\section{RESUMO}

Foram avaliados dois modelos para estimativa do potencial produtivo relativo de cana-de-açúcar. Utilizou-se dados de produtividade $\left(\right.$ t.ha $^{-1}$ ) de experimentos, dados de campo da Usina Santa Rita, localizado no município de Santa Rita do Passa Quatro e das áreas de cana-de-açúcar da Usina Costa Pinto em Piracicaba, todas localizadas no Estado de São Paulo, Brasil, para realizar uma comparação entre a produtividade obtida em campo e a produtividade relativa (\% da máxima produtividade) estimada pelo modelo matemático. As variáveis de entrada são o fósforo $(P, \mathrm{mmolc} / \mathrm{kg})$, potássio $(\mathrm{K}, \mathrm{mmolc} / \mathrm{kg})$, capacidade de troca de cátions $(\mathrm{T}, \mathrm{mmolc} / \mathrm{kg})$, saturação por bases $(\mathrm{V} \%)$ e o teor de argila 
(Arg\%). As avaliações mostraram que o modelo estimou de forma eficiente a produtividade relativa de cana-de-açúcar, com relações altamente significativas $(\mathrm{P}<0,001)$ entre produtividade medida no campo e produtividade relativa estimada. Verificou-se que o modelo ganha precisão quando se trabalha em escalas maiores (dados experimentais) em comparação com escalas menores (grandes fazendas). Uma análise de regressão linear múltipla, pelo método stepwise, foi utilizada para verificar quais foram os fatores que influenciaram a produtividade da cana-de-açúcar nos diferentes anos. Verificou-se forte influencia do número de cortes e das variedades, entretanto notou-se que as variáveis do solo utilizados como variáveis de entrada ( $P, K, T, V, A r g)$ nem sempre influenciaram a produtividade nos diferentes anos. Constatou-se então que as ferramentas estatísticas podem ser limitadas para elaboração de modelos. Consequentemente o modelo mostrou-se uma ferramenta útil para identificação da capacidade produtiva de áreas para a cana-de-açúcar de maneira holística e integrada em comparação aos métodos estatísticos. O modelo foi aplicado, numa escala regional, no o município de Piracicaba. Através de um sistema de informações geográficas (SIG) foram gerados um mapa de declividade, mapa de distância em relação a 4 usinas da região e uma mapa de produtividade relativa. Os mapas foram úteis na identificação da distribuição espacial da cana-de-açúcar no município. Utilizou-se o modelo climático da FAO para simular a produtividade da cana-de-açúcar mês a mês durante um ano. O mesmo não apresentou correlação com a produtividade média mensal obtida. Este modelo foi concebido para estudos em dimensões continentais com regiões climaticamente contrastantes. Em escala local se mostrou muito sensível ao déficit hídrico para estimar a produtividade da canade-açúcar adequado para realizar estimativas, principalmente porque esta cultura é tolerante a períodos de stress hídricos. 


\title{
EVALUATION AND APLICATION OF MODELS TO ESTIMATE SUGARCANE (Saccharum spp) PRODUCTION BASED ON SOIL AND WEATHER PARAMETERS
}

\author{
Author: EDSON ROBERTO TERAMOTO \\ Advisor: Prof. Dr. MARCOS SILVEIRA BERNARDES
}

\section{SUMMARY}

Two models of sugarcane were evaluated. The yield (t/ha) out of experimental data, and from fields of the Santa Rita Sugar Mill, located at Santa Rita do Passa Quatro, and from large farms of the Costa Pinto Sugar Mill, located at Piracicaba, all in the State of São Paulo - Brazil were compared to the estimated relative yield ( $\%$ of the maximum yield) by a mathematical model having as input the soil fertility parameters: potassium content $(\mathrm{K}$, in $\mathrm{mol} / \mathrm{kg})$, phosphorus content $(P$, in $\mathrm{mol} / \mathrm{kg})$, cation exchange capacity $(T$, in $\mathrm{mol} / \mathrm{kg})$, base saturation $(\mathrm{V}$, in \%) and clay content (Arg, in \%), by linear regression. The high significance of the regressions $(P<0,001)$ showed a closed relation between estimated and measured yield. The model gain precision with bigger scales (experimental data) compared to the smaller scales (larger farms). A linear 
multiple regression statistical analysis, by the stepwise method, were applied to select the soil parameter which most affected the yield. The ratton number and the sugarcane variety were the most important factors determining yield, overcoming the soil fertility parameters which not always affected yield according to this analysis. Consequently, the model was a better tool to identify soil capability for sugarcane yield, in a holistic and integrated manner, than the statistical analysis. The soil model to estimated relative yield were applied tin the regional scale on the Piracicaba county to generate maps of the distance of sugarcane field to the sugar mills, relative sugarcane yield of the fields, and land slopes of the sugarcane fields. The maps were useful to elucidate the sugarcane distribution within the county. An estimation of yield by the FAO model, using weather data as input parameters, showed a large month to month yield variation in discrepancy to the field observations. No significance regressions demonstrated that the yield estimated by FAO model is not related to the field data. This model was created for studies at continental level with contrasting weather differences, but is too sensitive for the water deficit to estimate sugarcane yield at local and regional scale, mainly because this crop is particularly tolerant do periodic water stress. 


\section{INTRODUÇÃO}

Assim como toda cultura agrícola, a produção da cana-de-açúcar (Saccharum spp) é influenciada por um grande número de fatores ambientais. Alguns desses não são passíveis de manejo, como o clima, enquanto outros, como o solo e a disponibilidade de água, podem ser manejados para permitir o melhor desempenho da cultura. A busca por altos rendimentos a baixos custos implica em conhecer mais detalhadamente o ambiente no qual a cultura está inserida, com o objetivo de racionalizar as relações entre os diferentes fatores de produção visando o máximo desempenho. Apesar da cultura da cana-deaçúcar ser uma das mais antigas atividades agrícolas do Brasil, vários trabalhos ainda têm sido desenvolvidos no intuito de estimar sua produtividade (Beauclair, 1991; Beauclair, 1994; Bittencourt et al., 1989; Humbert, 1968; Matioli, 1998; Penati 1991; Scarpari, 2002).

Das atividades econômicas, a agricultura é aquela com maior dependência das condições climáticas. As condições atmosféricas afetam todas as etapas das atividades agrícolas, desde o preparo do solo até a colheita, transporte e armazenamento dos produtos (Pereira et al., 2002). O clima, na escala regional, é o primeiro a ser considerado devido a sua condição de fator praticamente imutável (Alfonsi et al., 1987). Além disso, está entre os principais fatores determinantes da produtividade agrícola, e até hoje diversos estudos são conduzidos no intuito de relacionar parâmetros climáticos com a produtividade das culturas (Doorembos e Kassan, 1979).

Associado ao clima, as características físicas e químicas dos solos também são de grande importância. A análise de terra é, ainda hoje, reconhecida como a principal prática de avaliação do potencial de produtividade do solo sendo, portanto, uma das mais tradicionais formas de avaliação de sua 
capacidade produtiva (Bittencourt et al., 1989; Bittencourt et al., 1990; Lopes,1998; Malavolta, 1982; Orlando Filho et al., 1994; Raij, 1981; Raij et al.,1985).

Muitas pesquisas são realizadas com o objetivo de compreender as relações entre os diversos fatores de produção. Entretanto, grande parte destas só obtém resultados significativos a longo prazo e com grande demanda de recursos. Neste contexto, o uso de modelos de simulação assume grande importância em estudos com esse objetivo, caracterizando-se como importante ferramenta de assistência à tomada de decisões para viabilizar sistemas racionais de produção (Bernardes, 1999; Hanks \& Ritchie, 1991; Jones \& Kiniri, 1986; Pereira \& Machado, 1986; Thornley, 1976). A modelagem matemática é uma técnica que integra conhecimentos de diferentes disciplinas em uma estrutura de trabalho comum, podendo definir metas claras e comparar objetivos, muitas vezes conflitantes, permitindo a previsão do comportamento do sistema, o que é especialmente importante para situações ainda não experimentadas (Bernardes et al., 1998; Bernardes, 1999).

O objetivo geral deste trabalho foi testar modelos que simulam a produtividade da cana-de-açúcar com base em parâmetros climáticos e de análise de solo de rotina, comparando-se a produtividade medida no campo, com os resultados simulados através destes métodos.

As hipóteses deste trabalho são: (i) os resultados das análises de terra, agregados por um modelo matemático de produtividade relativa é um indicador da capacidade produtiva dos solos; (ii) os parâmetros climáticos rotineiros expressos através do modelo da FAO são um indicativo da capacidade produtiva da cana-de-açúcar em um dado período.

Os objetivos específicos deste trabalho são: (i) relacionar a produtividade da cana-de-açúcar com uma base de dados agronômicos simples, (ii) avaliar a aplicabilidade dos modelos propostos como ferramentas de planejamento e de suporte àdecisão em planejamentos agrícolas. 


\section{REVISÃO DE LITERATURA}

\subsection{Importância e panorama atual da cana-de-açúcar no Brasil:}

A cana-de-açúcar (Saccharum spp) pertence à família Poaceae, e é considerada originária do Sudeste Asiático, na grande região da Nova Guiné e Indonésia (Daniels \& Roach, 1987). Inicialmente cultivava-se principalmente a espécie Saccharum officinarum (L.), entretanto, os cultivares desta espécie passaram a sofrer dificuldades de adaptação ecológica e severos danos provocados por doenças. Híbridos inter específicos, oriundos dos programas de melhoramento genético, resistentes e melhor adaptados para diversas condições ambientais permitiram a expansão da cultura pelo mundo (Figueiredo et al., 1995; Matsuoka et al., 1999). A cana-de-açúcar é cultivada numa ampla faixa, desde $35^{\circ} \mathrm{N}$ a $30^{\circ} \mathrm{S}$, e em altitudes que variam do nível do mar a $1.000 \mathrm{~m}$ de altitude (Magalhães, 1987).

Ao final do século XX, o Brasil tornou-se o maior produtor mundial de cana-de-açúcar, de açúcar e de álcool (Balsadi et al., 1996). Essa liderança foi atingida principalmente em função da criação do Proálcool, em 1975, um programa governamental de incentivo à produção de álcool combustível (Leite, 1987).

Atualmente a cana-de-açúcar é uma das principais culturas agrícolas do país servindo como matéria prima para a produção de açúcar, álcool e alimentação animal. A área plantada com cana-de-açúcar no Brasil em 2001 foi de 5.022.187 de hectares, onde foram colhidos 344.281.802 toneladas, gerando uma receita bruta de $R \$ 8,68$ bilhões (IBGE, 2002). No Estado de São Paulo, em 2001 a área colhida foi de 2.568 .920 de hectares e a produção foi de 
201.682.870 de toneladas de cana-de-açúcar, gerando $\mathrm{R} \$ 5.34$ bilhões (IEA, 2002).

A região de Piracicaba é tradicionalmente produtora de cana-deaçúcar. Quase metade da área cultivada é ocupada com esta cultura que emprega a maior parte da mão-de-obra rural (Orplana, 1996). A cana-de-açúcar ocupava, em 1991, 49,3 \% da área total do município de Piracicaba, num total de 86.819 ha (Sparovek \& Lepsch, 1995; Sparovek et al., 1997a).

Devido a aspectos políticos, legais e tecnológicos, a prática da queimada prévia à colheita está sendo gradativamente eliminada, implicando em significativas transformações no sistema de produção canavieiro. A principal mudança tem sido a mecanização da colheita, que implica necessariamente em adaptações e modificações de outras etapas do sistema produtivo. A viabilidade da implantação da colheita mecanizada depende principalmente da declividade do terreno, sendo possíveis com até 12\% (Sparovek et al., 1997b), e da disponibilidade de capital para investimento do produtor, sendo economicamente viável em áreas contínuas com mais de 500 ha (Gonçalves \& Souza, 1998).

Tais mudanças possivelmente provocarão alterações no sistema produtivo da cana-de-açúcar bem como na dinâmica da ocupação e na avaliação das terras para essa cultura. Assim, em função da importância desta cultura, as transformações em curso terão impactos diretos sobre a qualidade de vida e os recursos naturais do Estado de São Paulo.

\subsection{Aspectos climáticos para a cultura da cana-de-açúcar}

A cana-de-açúcar, em função do seu ciclo perene, sofre a influência das variações climáticas durante todo o ano. Para atingir alta produção de sacarose, a planta precisa encontrar épocas com condições de temperatura e umidade adequadas para permitir o máximo crescimento na fase vegetativa, 
seguida de um período com restrição hídrica ou térmica para favorecer o acúmulo de sacarose no colmo na época do corte. A cana-de-açúcar encontra suas melhores condições quando ocorre um período quente e úmido, com intensa radiação solar durante a fase de crescimento, seguido de um período seco durante as fases de maturação e colheita. No Brasil, em função da sua extensão territorial, existem as mais variadas condições climáticas e possivelmente é o único país com duas épocas de colheita anuais: de setembro a abril no Norte e Nordeste e de maio a dezembro no centro-sul correspondendo às épocas secas nessas regiões (Alfonsi et al., 1987).

No que se refere aos fatores climáticos para a produção da cana-deaçúcar, a temperatura é, provavelmente, o de maior importância. A temperatura basal para a cana-de-açúcar está em torno de $20^{\circ} \mathrm{C}$. A temperatura ótima situase entre 22 a $30^{\circ} \mathrm{C}$, sendo que nestas condições a cultura apresenta seu máximo crescimento. Acima de $38^{\circ} \mathrm{C}$ não há crescimento (Barbieri \& Villa Nova,1977; Doorembos \& Kassan, 1979; Magalhães, 1987).

A cana-de-açúcar é considerada uma planta C4, com altas taxas fotssintéticas, apresentando alta eficiência de conversão de energia radiante em energia química. A radiação global que atinge a superfície da Terra é composta basicamente por duas partes: radiação solar direta e radiação difusa na atmosfera. Percentualmente, da radiação que atinge o topo da atmosfera, 25\% é radiação direta e $26 \%$ atinge a superfície como radiação difusa. Assim grande parte da radiação disponível para as plantas está na forma de radiação difusa. Os efeitos adicionais da radiação à fotossíntese sobre o desenvolvimento das plantas tornam mais complexas a análise da fotossíntese em relação à produtividade, e nem sempre a manipulação da radiação ou do sistema de captação de radiação visando aumentar a eficiência fotossintética leva a um incremento de produtividade agrícola (Bernardes, 1987).

Para a cana-de-açúcar, a relação entre taxa de fotossíntese, por unidade de área foliar por unidade de superfície de terreno, com a produtividade ainda depende de melhor entendimento dos aspectos fisiológicos relacionados 
a transporte e acumulação dos metabólitos, principalmente da sacarose. Para isso, estudos sobre as relações entre a eficiência dos sítios de produção (folhas) e dos sítios de acumulação (colmos) durante o crescimento da cultura devem ser realizados para serem relacionados com aspectos climáticos, principalmente no que se refere à captura da radiação pela planta (Magalhães, 1987).

\subsubsection{Consumo de água pela cana-de-açúcar}

Vários trabalhos têm sido conduzidos para estudar a influencia do clima na produtividade da cana-de-açúcar, principalmente envolvendo temperatura e disponibilidade de água (Dias et al., 1999; Matioli, 1998; Ometto, 1974; Ometto, 1980; Rojas ,1999; Scarpari, 2002; Thompson, 1976).

A disponibilidade de água para a planta de cana-de-açúcar é o principal fator climático causador de variabilidade, ano a ano, da sua produtividade. Entretanto, em função das variações locais de clima e de variedades, é difícil estabelecer uma relação entre produção e consumo de água pela cana-de-açúcar. Segundo Scardua \& Rosenfeld (1987) esse consumo também varia em função do estádio fenológico, do ciclo da cultura (cana planta ou cana soca), das condições climáticas, da água disponível no solo entre outros fatores. Esses autores apresentaram um resumo das determinações de consumo de água obtidas em campo (Tabela 1). 
Tabela 1. Consumo de água pela cana-de-açúcar, obtido em campo por diversos autores citados por Scardua \& Rosenfeld (1987).

\begin{tabular}{lcccccc}
\hline \multicolumn{1}{c}{ Autor } & Ano & Local & Ciclo da & \multicolumn{3}{c}{ Consumo de água (mm/dia) } \\
\cline { 5 - 7 } & & & Cultura & máximo & Mínimo & médio \\
\hline Cox & 1960 & EUA (Havaí) & -- & 8,0 & 3,8 & -- \\
Thompson & 1963 & África do Sul & -- & 6,0 & 2,2 & -- \\
Tosello & 1966 & Brasil & -- & 3,4 & 1,3 & -- \\
Cruciani & 1972 & Brasil & -- & 2,8 & 1,2 & -- \\
Souza & 1974 & Brasil & Planta & 4,5 & 2,3 & 3,6 \\
Souza & 1975 & Brasil & Soca & 5,0 & 2,2 & 3,6 \\
Leme & 1978 & Brasil & Planta & 5,6 & 1,9 & 3,8 \\
Leme & 1978 & Brasil & Planta & 4,8 & 1,3 & 3,2 \\
Scardua & 1979 & Brasil & Planta & 4,5 & 2,3 & 3,3 \\
Scardua & 1979 & Brasil & Soca & 4,4 & 2,2 & 3,2 \\
MÉDIA & & & & 4,9 & 2,1 & 3,5 \\
\hline
\end{tabular}

\subsubsection{Balanço hídrico e evapotranspiração}

$\mathrm{Na}$ interação do sistema solo-planta-atomosfera há uma certa quantidade de água que entra e sai de cada um desses componentes, fazendo com que a água armazenada no solo esteja em constante variação. Esta variação do armazenamento de água no volume de solo considerado por intervalo de tempo, representa o balanço do que entrou e do que saiu de água no sistema. $O$ estudo dessa contabilização da água no solo é chamado balanço hídrico $(\mathrm{BH})$, sendo o seu conhecimento muito importante, tanto na determinação imediata da necessidade hídrica de uma cultura quanto na tomada de decisões em projetos agrícolas. É caracterizado também como indicador do potencial climático de uma região para uma cultura qualquer (Ometto, 1989, Pereira et al., 2002).

Genericamente, as principais fontes de entrada de água no sistema são: (i) precipitação; (ii) orvalho; (iii) escorrimento superficial; (iv) drenagem 
lateral; (v) ascensão capilar e (vi) irrigação. As saídas principais são: (i) evapo(tranpi)ração; (ii) escorrimento superficial; (iii) drenagem lateral e (iv) drenagem profunda (Pereira et al., 2002).

Um dos métodos mais utilizados para calcular o $\mathrm{BH}$, é o de Thornthwait \& Mather (1955). Este método requer basicamente dados de evapotranspiração de referencia (ETP), ceficiente da cultura $(\mathrm{Kc})$ ao longo do período de desenvolvimento, precipitação $(\mathrm{P})$ e a capacidade de água disponível (CAD) para o período considerado. O termo evapotranspiração foi introduzido por Thornthwait na década de 40 para expressar o processo de transferência simultâneo da evaporação da água do solo e da transpiração das plantas numa superfície vegetada (Pereira et al., 2002).

A evapotranpiração potencial (ETP) é a quantidade de água que seria utilizada numa superfície vegetada com grama com plena disponibilidade de água no solo. A evapotranspiração nessas condições é tomada como referencia quando se quer conhecer a evapotranspiração de uma cultura, em condição não padrão. Assim a evapotranspiração potencial é um valor indicativo da demanda evapotranspirativa da atmosfera de um local em um dado período. A evapotranpiração real (ETR) é a quantidade de água realmente utilizada por uma superfície vegetada com grama porém com ou sem restrição hídrica. Quando não há restrição $E T P=E T R$, portanto ETR $\leq E T P$. Os conceitos de ETP e ETR são aplicados somente a uma superficie gramada, portanto não faz sentido referir-se a evapotranspiração potencial de uma cultura (Pereira et al., 1997).

A evapotranspiração da cultura (ETc) é a quantidade de água utilizada por uma cultura, em qualquer fase de seu desenvolvimento, desde 0 plantio até a colheita, quando não houver restrição hídrica. A ETc é função principalmente do IAF. A ETc pode ser obtida pelo produto entre ETP e um coeficiente de cultura $(\mathrm{Kc})$. O valor de $\mathrm{Kc}$ varia com as fases fenológicas, e também entre espécies e variedades (Pereira et al., 2002). 
O método de estimativa de Thornthwait, apesar de utilizar somente a temperatura do ar como variável independente é considerado um método adequado na determinação da evapotranspiração potencial, tanto na freqüência mensal como diária, para um clima como o do Estado de São Paulo (Pereira et al., 2002).

Para estimar a evapotranspiração da cultura é preciso ajustar o ETP através do coeficiente de cultura Kc. Os coeficientes de cultura para os diferentes estádios de desenvolvimento da cana-de-açúcar, foram definidos por Doorenbos \& Pruitt (1977) e Doorenbos \& Kassan (1979), que são os coeficientes preconizados pela FAO (Tabela 2).

Tabela 2. Coeficientes de cultura para cana-de-açúcar, de acordo com Doorenbos \& Pruitt (1977) e Doorenbos \& Kassan (1979).

\begin{tabular}{|c|c|c|c|c|c|}
\hline \multicolumn{2}{|c|}{ Idade da cultura (meses) } & \multirow{2}{*}{$\begin{array}{c}\text { Período de } \\
\text { Desenvolvimento }\end{array}$} & \multicolumn{3}{|c|}{ Coeficiente (Kc) } \\
\hline cana planta & cana soca & & mínimo & máximo & Médio \\
\hline $0-2$ & $0-1$ & Do plantio até $25 \%$ de cobertura do solo ${ }^{(1)}$ & 0,40 & 0,60 & 0,50 \\
\hline $2-3$ & $1-2$ & De $25 \%$ a $50 \%$ de cobertura do solo ${ }^{(1)}$ & 0,75 & 0,85 & 0,80 \\
\hline $3-4$ & $2-3$ & De $50 \%$ a $75 \%$ de cobertura do solo ${ }^{(1)}$ & 0,90 & 1,00 & 0,95 \\
\hline $4-7$ & $3-4$ & De $75 \%$ a cobertura completa do solo ${ }^{(1)}$ & 1,00 & 1,20 & 1,10 \\
\hline $7-14$ & $4-9$ & Utilização máxima ${ }^{(2)}$ & 1,05 & 1,30 & 1,20 \\
\hline $14-16$ & $9-10$ & Início da maturação ${ }^{(3)}$ & 0,80 & 1,05 & 0,95 \\
\hline $16-18$ & $10-12$ & Maturação ${ }^{(3)}$ & 0,60 & 0,75 & 0,70 \\
\hline $\begin{array}{l}\text { (1) Primeir } \\
\text { (2) Seguno } \\
\text { (3) Terceir }\end{array}$ & $\begin{array}{l}\text { dio: forr } \\
\text { dio: mat }\end{array}$ & $\begin{array}{l}\text { da cultura } \\
\text { dução }\end{array}$ & & & \\
\hline
\end{tabular}

Doorembos \& Kassan (1979), definiram três estádios para a cana-deaçúcar: (i) estabelecimento da cultura, seguido de um período vegetativo, (ii) formação da produção e (iii) maturação. Para cana-de-açúcar, o primeiro estádio é o mais sensível à deficiência hídrica (Doorembos \& Pruitt, 1977; Rosenfeld, 1989; Thompson, 1976). 


\subsection{Relações entre solos e produção da cana-de-açúcar}

O conhecimento da capacidade produtiva dos solos é um dos principais fatores de sucesso do empreendimento agrícola. Portanto o conhecimento das características inerentes a cada solo, os chamados fatores edáficos, é importante para julgar o seu potencial de produção agrícola (Lepsch, 1987).

Cada solo apresenta um potencial de produção agrícola para a canade-açúcar, mas estes, somados às carac terísticas da paisagem na qual estão inseridos, podem compor ambientes com potenciais diferenciados para a produção dessa cultura. O agrupamento de solos ou de ambientes edáficos é possível, e em muitos casos conveniente, como demonstrou Joaquim et al. (1994) ao estabelecer uma classificação preliminar do potencial produtivo dos solos, agrupando-os de acordo com o nível de produção da cana-de-açúcar.

A bibliografia sobre relações entre propriedades e características do solo e produtividade da cana-de-açúcar é vasta. Inúmeros trabalhos contemplam este tema (Beauclair, 1994; Bittencourt et al., 1989; Bittencourt et al., 1990; Dias et al., 1999; Humbert, 1968; Maule, 2001; Nunes Júnior, 1987; Orlando Filho, 1983; Orlando Filho et el., 1990). Alguns porém, têm resultados com validade local, restringindo sua aplicabilidade ao ambiente onde foi conduzido cada experimento.

A cana-de-açúcar apresenta uma grande adaptabilidade, podendo ser cultivada tanto em solos arenosos, que apresentam como limitações a baixa capacidade de armazenamento de água e alta taxa de perda de nutrientes por lixiviação, até em solos muito argilosos que podem apresentar uma drenagem mais lenta, propiciando menor aeração da zona radicular, e também alta suscetibilidade à compactação, que é li mitante à penetração das raízes (Kofler \& Donzeli, 1987). Entretanto cada solo apresentará vantagens e limitações, inerentes a sua classificação, que devem ser considerados quando utilizados para a cana-de-açúcar. 
Penatti (1991), também observou que o potencial produtivo é diferente entre solos de textura arenosa, média e argilosa, sendo que os solos argilosos possuem maior potencial.

Apesar da quantidade de trabalhos sobre relações entre solos e produtividade, ainda é difícil gerar, de forma segura, para uma dada região, uma estimativa preliminar de produtividade de cana-de-açúcar tendo como base os parâmetros do solo. Relações mais abrangentes devem ainda ser implementadas para possibilitar extrapolações regionais, e dessa forma subsidiar trabalhos de planejamento voltados para a cultura da cana-de-açúcar .

\section{$2.4 \quad$ Modelos e simulações}

Os conceitos de modelos e simulações surgiram da necessidade inerente do homem em compreender como a natureza e seus componentes funcionam. Para isso, foi necessário isolar o objeto de estudo, bem como parte do ambiente que interfere no mesmo, uma vez que na natureza os objetos são interdependentes e se influenciam mutuamente.

Esse conjunto funcional, formado pelo objeto alvo contido nessa parte do ambiente, constitui um sistema que, geralmente está inserido num sistema maior e mais complexo. Assim, um sistema é uma parte da realidade com elementos interrelacionados e com limites definidos, de forma que 0 ambiente exerça uma certa influência sobre o sistema, mas este deve ter pouca ou nenhuma influência no ambiente (Penning de Vries, 1982; Thornley, 1976; de Wit \& Goudriaan, 1974; de Wit, 1978).

Um sistema, de qualquer natureza, também pode ser estudado pela distinção e caracterização de seus componentes principais, ou através de equações matemáticas que representem as mudanças internas, externas e suas inter-relações com o ambiente de tal forma que se caracterizem num modelo do sistema original. Assim pode-se definir uma modelo como sendo 
uma representação simplificada de uma sistema (Hanks \& Ritchie, 1991; Laffelaar, 1993).

Existem vários tipos de modelos, que podem ser expressos de várias formas. Os modelos empíricos consistem em analisar dados experimentais, realizando algumas análises dos mesmos e tentando idealizar equações matemáticas que possam ser usadas como modelos matemáticos que se ajustem aos dados observados. Nesse tipo de modelo utilizam-se dados empíricos, variáveis ou constantes consagradas que fazem parte do conhecimento de determinada ciência. Quando o interesse é descrever primariamente a resposta de um sistema de forma geral, o método empírico pode ser utilizado satisfatoriamente. Neste caso não há muito interesse em explicar detalhadamente porque o sistema responde de tal forma. Os modelos mecanísticos tenta entender um sistema em termos dos mecanismos presentes. Assim um modelo pode ser construído com base na estrutura do sistema, dividindo o sistema em componentes e tentando entender o comportamento do sistema como um todo em termos do comportamento dos componentes individuais e suas interrelações. Um modelo é chamado determinístico se o estado do sistema num momento for determinado, exclusivamente, pelo estado do sistema no momento anterior (Patten,1971). O modelo é estocástico se o estado do sistema for determinado pelo momento anterior afetado por uma probabilidade devida ao acaso São modelos que contém alguns elememtos que variam ao acaso e que fazem parte do modelo. São modelos em que o fator probabilidade está presente (Thornley, 1976).

$\mathrm{Na}$ modelagem, sem dúvida, a face mais importante é que essa técnica possibilita o entendimento de um sistema de forma integral e holística, e a simulação, por outro lado, estimula e aumenta a compreensão da realidade através de analogias e extrapolações quando o sistema estudado é muito complexo (Bos \& Rabbinge, 1976; Thornley, 1976).

O enfoque holístico, essencial ao estudo de sistemas agrícolas e ecológicos de forma geral, permite a identificação de sistemas, subsistemas e 
dos processos mais importantes, com atenção especial na interação entre estes. Ainda que olhando para todo o sistema, pode-se otimizar o enfoque tradicional da pesquisa monodisciplinar, e auxiliar $o$ incremento na produtividade e na sustentabilidade de sistemas agrícolas (Penning de Vries, 1982).

Os sistemas biológicos são complexos e podem ser estudados em vários níveis de organização. Sistemas naturais que envolvem vários outros sistemas recebem um nome geral de ecossistemas, que se caracterizam essencialmente pela sua grande complexidade. A análise dos sistemas ecológicos parte da premissa de que o estado de qualquer sistema, em qualquer momento pode ser caracterizado quantitativamente e suas mudanças podem ser descritas através de equações matemáticas. Essa hipótese leva a formulação de modelos determinísticos, no qual as variáveis de estado, taxas e variáveis indutoras podem ser discriminadas (de Wit, 1974).

Para entender o comportamento de determinado nível do sistema, muitas vezes é necessário compreender como este é influenciado pelo comportamento de um nível abaixo. Assim um sistema aparentemente complexo pode ser desdobrado em níveis relativamente mais simples (Jones, 1986; de Wit, 1974).

Quando o conhecimento de determinado nível explicativo é suficientemente extensivo, tendo sido o modelo deste sistema criado com base nesse sólido conhecimento, pode não ser mais necessário testar esses modelos comparando-os com a realidade (de Wit, 1974).

\subsubsection{Modelagem em sistemas agrícolas}

Os diferentes modelos de culturas agrícolas, com maior ou menor nível de empirismo em função do conhecimento sobre os fenômenos estudados, normalmente consistem no balanço do carbono, que depende da interceptação e da utilização da luz, e de um balanço hídrico, que depende da demanda atmosférica e da utilização da água pelas planta. Estes processos 
fisiológicos podem ser associados à absorção dos nutrientes e às perdas causadas por pragas, doenças e plantas daninhas. A agregação destes conhecimentos através de equações matemáticas, resulta em modelos que podem simular o crescimento e a produção das culturas (Penning de Vries, 1982).

Muitos modelos de crescimento de plantas baseiam-se na simulação da produção fotossintética e da partição dos fotossintetizados para seu crescimento, armazenamento e respiração (Barbieri, 1993). A pesquisa da fotossíntese é refletida em sofisticados modelos, os quais existem para predizer o crescimento das plantas, dados da elevação solar, geometria das folhas, penetração da luz, taxa individual da fotossíntese, etc.

O crescimento e desenvolvimento das plantas dependem fundamentalmente dos processos de fotossíntese e respiração. A maioria dos recursos e esforços, no entanto, tem sido despendidos no estudo da fotossíntese, sendo a respiração considerada apenas um processo de perda de carbono (Pereira \& Machado, 1987). Coube a McCree (1970) e Thornley (1970), mostrar que esses dois processos são interligados e interdependentes, e que a respiração faz parte efetiva do processo de utilização e distribuição dos carboidratos fotossintetizados.

De modo geral algumas das contribuições desses modelos à pesquisa agrícola, educação e planejamento, apontados por diversos autores (France \& Thornley, 1984; Hanks \& Ritchie, 1991; Jones, 1986) são: (i) fornecer um panorama coerente sobre o desempenho de todo o sistema; (ii) formular uma descrição precisa sobre novas hipóteses geradas, as quais devem ser testadas para ajudar a definir áreas onde o conhecimento é limitante; (iii) estimular uma integração interdisciplinar e descrever de forma concisa um grande número de observações isoladas, e (iv) predizer o comportamento do sistema em combinações e condições não experimentadas anteriormente.

A elaboração dos modelos matemático-fisiológicos também oferecem, segundo Thornley (1976) uma série de vantagens: (i) informações a 
respeito de diferentes processos fisiológicos podem ser reunidas em um único modelo, para se ter idéia da cultura como um todo; (ii) um modelo resume convenientemente grande quantidade de informações; (iii) a base matemática para as hipóteses adotadas permite compreender quantitativamente a natureza das interações ambiente-planta; (iv) a modelagem pode estimular novas idéias; (v) a elaboração de um modelo ajuda a detectar áreas onde o conhecimento é limitado; e (vi) modelos permitem interpolações e previsões.

No atual estado de desenvolvimento dos modelos para a agricultura, os módulos de simulação dos processos componentes parecem ser aqueles que mais rapidamente podem ser incorporados e utilizados, como os que, por exemplo, simulam o balanço hídrico para orientação do manejo da irrigação.

Assim, os modelos matemáticos sintetizam, apresentam e analisam diversos aspectos da produção agrícola, tais como arranjo espacial entre plantas, diversos tipos de interações entre as culturas e o ambiente, características físicas e químicas de solos, otimização do uso de equipamentos e máquinas, sistemas de transportes e ainda modelos sócio-econômicos. Os modelos são ferramentas essenciais para realização de estimativas e extrapolações de situações, que são importantes etapas no processo de planejamento que por sua vez não podem se fundamentar exclusivamente em ensaios de campo para sua execução (Bernardes, 1999).

O progresso obtido através do uso de modelos é mais rápido e de menor custo que a pesquisa experimental isolada, ainda que os principais resultados tenham que continuar a serem avaliados com experimentos. Também já demonstraram ser eficientes para a determinação de prioridades na alocação de fundos para pesquisas e na escolha da melhor cultura para programas de desenvolvimento agrícola (Hanks \& Ritchie, 1991). Os modelos já existentes podem ser associados a outros ou modificados para aproximá-los da realidade e da necessidade de informação (Pereira \& Machado, 1986).

A construção de modelos simplificados, ou sumarizantes, é interessante para torná-los mais acessíveis ao público não especializado. Tais 
modelos podem ser feitos extraindo-se todos os detalhes excessivos do modelo complexo, usando-se análises de sensibilidade e pela seleção da variável principal do sistema. Diferentes modelos simplificados podem ser construídos a partir de um mesmo modelo complexo, as diferenças entre eles irá depender dos diferentes objetivos de sua aplicação. (Bernardes, 1999)

\subsubsection{Modelos em cana-de-açúcar}

Pereira (1987), e Pereira \& Machado (1986) descreveram um modelo matemático de balanço de carbono, onde integrou três processos que normalmente são estudados em separado: crescimento, fotossíntese e respiração, em uma única equação, em torno da qual foi idealizado o modelo matemático do crescimento e desenvolvimento da cana-de-açúcar.

Inman-Bamber \& Thompson (1989) desenvolveram um modelo de acúmulo de matéria seca. Neste modelo foram utilizados dois modelos para entender os efeitos da idade e do clima na produção de matéria seca da canade-açúcar, utilizando-se água e fertilizantes em quantidades adequadas. O segundo modelo, chamado "Glover model", é a combinação do modelo de respiração de Glover (1972), com o modelo de fotossíntese CERES-MAIZE (Jones and Kiniry, 1986).

Barbieri (1993) desenvolveu um modelo matemático-fisiológico de estimativa de produtividade da cana-de-açúcar. É composto por uma série de equações que descrevem o comportamento fisiológico da planta em resposta as condições ambientais, sendo que as constantes das equações foram obtidas adaptando-se os resultados de pesquisas disponíveis na literatura.

O modelo CANEGRO, descrito por Inman-Bamber (1991) consiste em balanços detalhados do carbono e da água em uma seleção de equações do balanço de energia para determinar a exigência de água da cultura. Esse modelo explica o balanço de água, energia e carbono em três diferentes compartimentos. 
O modelo CANESIM (Singels et al., 1998) desenvolvido na África do Sul, evoluiu de um modelo proposto para controle de irrigação da cana-deaçúcar, o IRRICANE (Singels et al., 1989), com a inclusão de rotinas para simular a produtividade e também de um modelo para simular a área foliar da cultura. Este modelo foi desenvolvido com os mesmos conceitos básicos do modelo CANEGRO, entretanto mais simples e com menor quantidade de parâmetros de entrada. Apesar de não ser tão robusto quanto o CANEGRO, o CANESIM pode ser utilizado por usuários tanto na indústria como no campo gerando resultados confiáveis. O desenvolvimento do CANESIM ocorreu principalmente em função da demanda por informações no setor produtivo em suas atividades de planejamento. O modelo CANEGRO não atendia essa demanda porque exige uma quantidade excessiva de parâmetros de entrada, tornando-o inviável para usos aplicados e práticos que necessitam de respostas a curto prazo.

\subsection{Avaliação de terras, suporte à decisão e planejamento regional}

A avaliação de terras pode ser definida como o processo de análise do desempenho da terra quando usada para um propósito especifico, ou como um método empregado para predizer o potencial de uso das terras (Rossiter, 1996).

A evolução tecnológica na agricultura e no estudo das ciências ambientais criou uma tendência crescente na busca de métodos que respondam a questões cada vez mais especificas para os diversos problemas relacionados a estas duas áreas da ciências. Tal fato constitui uma tendência que faz com que a maioria dos recentes trabalhos direcionem-se para as avaliações quantitativas, buscando valores numéricos que expressem e caracterizem fenômenos que ocorrem em campo, principalmente pelo uso de modelos matemáticos. Por outro lado, a aplicação de modelos requer normalmente um grande número de informações a serem levantadas, frutos de 
diversos experimento de campo, envolvendo um elevado custo e tempo para sua obtenção. Com a escassez de dados, os modelos tornam-se pouco confiáveis (Câmara, 1993).

O procedimento adotado tem sido o de quantificar os parâmetros de análise de maneira que estes possam ser utilizados nos cálculos dos modelos matemáticos (Bouma, 1997), incluir indicadores de sustentabilidade como parte dos procedimentos de avaliação (Hurni, 2000, Sparovek, 2002) e aumentar a adoção de suportes informatizados como, por exemplo, sistemas de informação geográficos, bancos de dados eletrônicos e sistemas especialistas e de suporte a decisão.

Um dos mais importantes sistemas de avaliação do potencial de terras foi desenvolvido pela FAO e é denominado "Framework for land Evaluation" (FAO, 1976). Novas aproximações tem sido desenvolvidas, com caráter mais quantitativo, contemplando inclusive variáveis sociais e econômicas em modelos de predição (Diepen et al., 1991).

A existência de modelos complexos, quantitativamente avaliados em condições experimentais controladas, não garante que esses serão aplicáveis em condições práticas em escala regional. A falta de dados específicos, conhecimentos insuficientes para entender como os modelos trabalham ou como interpretar resultados podem restringir a sua adoção. Em outros casos, a falta de tempo para obter e fornecer os dados, testar o modelo e interpretar os resultados, até mesmo com dados existentes, mantém a tecnologia de avaliação de terra moderna muito mais no ambiente acadêmico e experimental do que numa esfera prática de apoio á decisão. Estas dificuldades certamente são mais limitantes em países em desenvolvimento e regiões com economias em transição quando comparado com regiões industrializadas (Chinene, 1991).

Assim, identifica-se uma carência de modelos menos complexos, com necessidade de poucos parâmetros de dados de entradas ou que utilize parâmetros rotineiros e que permitam responder a questões básicas de planejamento agrícola a curto prazo. 
No presente estudo faz-se um exercício de utilização de parâmetros de análises de solo e de dados climáticos rotineiros com o intuito de alimentar modelos matemáticos simples de previsão de produtividade agrícola. 


\section{MATERIAL E MÉTODOS}

\subsection{Localização das áreas com cana-de-açúcar}

Neste trabalho foram utilizados dados de produtividade de cana-deaçúcar (t.ha ${ }^{-1}$ ) provenientes de várias localidades do Estado de São Paulo.

$\mathrm{Na}$ fase de testes do modelo de estimativa de produtividade relativa de cana-de-açúcar primeiramente foram utilizados dados de produtividade dos experimentos realizados por Dias et al. (1999) conduzidos em Valparaiso $\left(50^{\circ} 52^{\prime} \mathrm{W}, 21^{\circ} 17^{\prime} \mathrm{S}\right)$, Estrela D'Oeste $\left(50^{\circ} 50^{\prime} \mathrm{W}, 20^{\circ} 187^{\prime} \mathrm{S}\right)$, Clementina $\left(50^{\circ} 12^{\prime} \mathrm{W}, 21^{\circ} 30^{\prime} \mathrm{S}\right)$ e Araçatuba $\left(50^{\circ} 20^{\prime} \mathrm{W}, 21^{\circ} 15^{\prime} \mathrm{S}\right)$, e por Maule et al. (2001) conduzido em Castilho (51 $\left.35^{\prime} \mathrm{W}, 20^{\circ} 40^{\prime} \mathrm{S}\right)$.

Em seguida foram utilizados dados obtidos em alguns talhões das áreas de produção de cana-de-açúcar da Usina Santa Rita S.A. localizada em Santa Rita do Passa Quatro ( $\left.47^{\circ} 30^{\prime} \mathrm{W}, 21^{\circ} 50^{\prime} \mathrm{S}\right)$.

E finalmente foram utilizados dados de produtividade obtidos das áreas de produção de cana-de-açúcar da Usina Costa Pì nto, Grupo Cosan S.A., que se localizam entre as latitudes $22^{\circ} 30^{\prime}$ e $22^{\circ} 50^{\prime}$ sul e entre as longitudes de $47^{\circ} 40^{\prime}$ e $47^{\circ} 50^{\prime}$ oeste, no município de Piracicaba. Este trabalho foi desenvolvido com maior ênfase nos dados desta usina.

\subsection{Dados de produtividade e de análise de terra}

Os dados de produtividade de Dias et al. (1999) referem-se àcolheita de 1995, de Maule et al. (2001) do ano de 1998, da Usina Santa Rita do ano de 
2000. Na Usina Costa Pinto utilizou-se os dados de produtividade da colheita de 1999, 2000 e 2001.

Foram utilizados os resultados das análises de terra das camadas de 0-25 e 25-50cm, analisadas segundo Raij et al. (1987) de todos os locais considerados. Na Usina Costa Pinto foram utilizados os dados das análises de solo dos anos de 1999 e 2000.

$\mathrm{Na}$ Usina Santa Rita utilizaram-se dados de 23 talhões com cana de primeiro corte, na Usina Costa Pinto utilizaram-se apenas dados de talhões com cana a partir do segundo corte. No ano de 1999 foram utilizados dados de 134 talhões totalizando uma área de 1.294,65ha. No ano de 2000 foram utilizados 176 talhões somando $1.843,97$ ha.

\subsection{Dados Climáticos}

Foram utilizados os dados climáticos obtidos na estação meteorológica automática do Departamento de Ciências Exatas da ESALQ para elaboração do balanço hídrico bem como para os cálculos necessários para a utilização do modelo da FAO que foi avaliado somente no município de Piracicaba.

\subsection{Modelo para estimativa da produtividade relativa baseado em parâmetros do solo}

O modelo matemático para estimativa de produtividade relativa de cana-de-açúcar baseado em parâmetros do solo foi inicialmente proposto e utilizado por Levy (1995), entretanto de forma teórica, sem comparação com valores de produtividade real. 


\subsubsection{Base teórica}

Segundo Malavolta (1976), a ciência da fertilidade do solo e da nutrição de plantas tiveram nos trabalhos de Liebig um divisor de águas. Durante mais de meio século os fatores de produção foram encarados através da lei do mínimo, ou lei de Liebig, segundo a qual o crescimento da planta é limitada por aquele nutriente que ocorre em menores proporções e ele seria o único a limitar a produção. Segundo Brady (1983) e Epstein (1975) a principal contribuição de Liebig foi a de ter introduzido o conceito de que o solo contribui com constituintes inorgânicos solúveis, derrubando a teoria humista que considerava a matéria orgânica como a única fornecedora de carbono æ̀ plantas. Essa teoria revolucionou e influenciou sobremaneira o pensamento relacionado com a adubação e nutrição de culturas. Embora a chamada lei tenha aplicação limitada, a sua idéia básica não deve ser perdida de vista. (Raij, 1981).

A lei dos incrementos decrescentes, ou lei de Mitscherlich, veio complementar a de Liebig (Malavolta, 1976). De acordo com essa lei a adição de quantidades sucessivas de um nutriente resulta em acréscimos menores na produção. Posteriormente trabalhos envolvendo curvas de respostas a nutrientes em diversas culturas foram realizados em grande quantidade. Neste contexto vários trabalhos exemplificam a importância dessa teoria, dentre os mais abrangentes pode-se citar, Haag et al., 1987; Malavolta et al., 1974, Malavolta, 1976; Malavolta, 1982; 1990 e Raij, 1985.

Recentemente conceitos de produção relativa (ou porcentagem de suficiência), segundo o qual a produção final é o resultado do produto dos diferentes fatores envolvidos e não a resultante de um fator único, foram introduzidos em estudos sobre fatores de produção e rendimento de culturas. (Raij, 1981).

Outro conceito que relaciona fatores de produção e produtividade relativa é o índice de rendimento (ou de produtividade) do solo. Foi proposto e 
utilizado por Sparovek et al. (1993) para avaliar a produtividade de uma cultura em função da profundidade de remoção de terra por erosão. Esse conceito se aplica a outras situações em que se deseja estimar a produtividade de uma cultura em função de parâmetros do solo.

O modelo proposto baseia-se nos conceitos teóricos acima expostos, e com isso pressupõe-se que o mesmo está adequadamente embasado.

\subsubsection{Parâmetros utilizados e entrada de dados}

Como entrada de dados do modelo de estimativa de produtividade relativa com base em parâmetros do solo necessita que cada talhão possua análise de solo em duas profundidades. Os parâmetros da análise de solo são o potássio $(\mathrm{K}, \mathrm{mmolc} / \mathrm{kg})$, fósforo $(\mathrm{P}, \mathrm{mmolc} / \mathrm{kg})$, capacidade de troca de cátions ( $\mathrm{T}, \mathrm{mmolc} / \mathrm{kg}$ ), saturação por bases (V\%) e o teor de argila (\%).

Estes parâmetros foram escolhidos em função de trabalhos que relacionam níveis de produtividade à parâmetros de fertilidade do solo (Bittencourt et al., 1990; Haag et al., 1987; Lopes, 1998 Malavolta et al., 1974, Malavolta, 1976; Malavolta, 1982; Orlando Filho et al., 1990; Raij, 1985).

Com relação ao teor de argila, considera-se que solos muito argilosos podem apresentar algumas limitações de natureza física como drenagem mais lenta, menor aeração da zona radicular, e alta suscetibilidade à compactação. Entretanto, grande parte dos solos ocupados com cana-deaçúcar são latossolos argilosos. Neste caso, o alto teor de argila não se manifesta claramente como depreciador pois esta classe possui uma série de vantagens decorrentes de suas características físicas.

Cada parâmetro foi dividido em 3 classes de teores, que são intervalos médios utilizados para fins de interpretação e classificação dos teores no solo (Malavolta, 1982; Raij, 1985). Para cada intervalo de classe foi estabelecida uma pontuação, que por sua vez se relaciona ao teor do parâmetro no solo. Assim cada parâmetro recebe uma pontuação em função de seu teor no solo. Acima destes valores espera-se uma produtividade ideal, 
desde que satisfeitas todas as outras necessidades da cultura. A Tabela 3 apresenta os parâmetros, os intervalos de classes e as respectivas pontuações.

Tabela 3. Intervalo de classes dos parâmetros do solo e o respectivo intervalo de pontuação.

\begin{tabular}{ccccccc}
\hline & \multicolumn{5}{c}{ Classes de teores dos parâmetros do solo } \\
Intervalo de & $\mathrm{P}$ & $\mathrm{K}$ & $\mathrm{T}$ & $\mathrm{V}$ & $\mathrm{Arg}$ \\
Pontuação & $\mathrm{mg} \cdot \mathrm{kg}^{-1}$ & \multicolumn{2}{c}{ mmolc.kg $^{-1}$} & & $\%$ & \\
\hline 1 & $\geq 35$ & $\geq 6,0$ & $\geq 125$ & $\geq 75$ & $\geq 50$ \\
$0,51-1,0$ & $15,1-35$ & $3,0-6,0$ & $75,1-125$ & $50,1-75$ & $25,1-50$ \\
$0,26-0,50$ & $6,1-15,0$ & $1,6-3,0$ & $50,1-75$ & $35,1-50$ & $12,1-25$ \\
$0-0,25$ & $0-6,0$ & $0-1,5$ & $0-50$ & $0-35$ & $0-12$ \\
\hline
\end{tabular}

Como a pontuação é função do teor no solo de cada parâmetro, foram determinadas equações em cada intervalo de classe, onde $\mathrm{Y}$ é a pontuação e Xp, Xk, Xt, Xv, Xa são os teores de K, P, T, V\%, e argila no solo, respectivamente. As equações são apresentadas na Tabela 4.

Tabela 4. Equações para determinação da pontuação em cada classe de intervalo de pontuação em função dos teores no solo de cada parâmetro.

\begin{tabular}{clll}
\hline & \multicolumn{3}{c}{ Intervalo de pontuação } \\
Parâmetro & $0-0,25$ & $0,26-0,50$ & $0,51-1,0$ \\
\hline$P$ & $\mathrm{Y}=0,042 \mathrm{Xp}$ & $\mathrm{Y}=0,028 \mathrm{Xp}+0,083$ & $\mathrm{Y}=0,025 \mathrm{Xp}+0,125$ \\
$\mathrm{~K}$ & $\mathrm{Y}=0,167 \mathrm{Xk}$ & $\mathrm{Y}=0,167 \mathrm{Xk}$ & $\mathrm{Y}=0,167 \mathrm{Xk}$ \\
$\mathrm{T}$ & $\mathrm{Y}=0,005 \mathrm{Xt}$ & $\mathrm{Y}=0,010 \mathrm{Xt}-0,250$ & $\mathrm{Y}=0,010 \mathrm{Xt}-0,250$ \\
$\mathrm{~V}$ & $\mathrm{Y}=0,007 \mathrm{Xv}$ & $\mathrm{Y}=0,017 \mathrm{Xv}-0,333$ & $\mathrm{Y}=0,020 \mathrm{Xv}-0,500$ \\
Arg & $\mathrm{Y}=0,021 \mathrm{Xa}$ & $\mathrm{Y}=0,019 \mathrm{Xa}-0,019$ & $\mathrm{Y}=0,020 \mathrm{Xa}$ \\
\hline
\end{tabular}


A pontuação do solo é composta pelas seguintes relações:

$$
\begin{aligned}
& P_{1}=P p_{1}+P k_{1}+P t_{1}+P v_{1}+P a_{1} \\
& P_{2}=P p_{2}+P k_{2}+P t_{2}+P v_{2}+P a_{2} \\
& P s=P P_{1}+P_{2}
\end{aligned}
$$

Onde:

Pp; Pk; Pt; Pv; Pa = pontuações parciais dos teores de P, K, T, V, argila das camadas 1 e 2

$\mathrm{P}_{1}=$ pontuação da camada $1(0-25 \mathrm{~cm})$

$\mathrm{P}_{2}=$ pontuação da camada $2(25-50 \mathrm{~cm})$

$\mathrm{Ps}=$ pontuação do solo

A estimativa da produtividade relativa é dada pela seguinte equação:

$$
\text { P rel }=\frac{\left(P s-P_{\min }\right) \times F}{\left(P_{\max }-P_{\min }\right)}+(100-F)
$$

Onde:

$\mathrm{P}$ rel $=$ Produtividade relativa estimada

$\mathrm{P}_{\text {min }}=$ pontuação mínima do conjunto de dados

$\mathrm{P}_{\max }=$ pontuação máxima do conjunto de dados

$\mathrm{F}=$ fator ponderador do solo

A produtividade estimada em t.ha ${ }^{-1}$, é obtida multiplicando-se a produtividade relativa pela máxima produtividade observada na população de talhões ou unidade de área do canavial do estudo em questão, submetida æ̀ mesmas condições climáticas e com a mesma idade. Assim tem-se a equação abaixo:

$$
\mathrm{TCH}_{\mathrm{e}}=\mathrm{P} \text { rel } x \text { P máxima observada }\left(\text { t.ha }^{-1}\right)
$$




\subsubsection{Testes do modelo para estimativa da produtividade relativa baseado em parâmetros do solo}

Foram realizados testes para avaliar o modelo em três situações e escalas de detalhe diferentes. Testou-se inicialmente o modelo, numa escala de maior detalhe, utilizando dados experimentais obtidos com controle rigoroso, de Dias et al. (1999) e Maule et al. (2001). Em seguida utilizou-se o modelo numa escala intermediária, em condições semi controladas na usina Santa Rita, em trabalho previamente descrito por Bernardes et al. (2002), onde obteve-se dados de produtividade, análise de solos e variedades de alguns talhões. E finalmente testou-se o modelo com dados da usina Costa Pinto, numa escala de menor detalhe, porém operacionalmente maior.

\subsubsection{Aplicação do modelo em escala regional}

Após os testes em diferentes escalas, o modelo foi aplicado para estimativa de produção em escala regional, no município de Piracicaba. Para isso utilizou-se os dados analíticos dos solos da carta pedológica semidetalhada da quadrícula de Piracicaba, escala 1:100.000 (Oliveira \& Prado, 1989). Entretanto os levantamento pedológicos não realizavam a determinação analítica de $\mathrm{P}$, pois esse elemento não era utilizado para fins de classificação. $\mathrm{O}$ teor de fósforo foi calculado então com base no teor de $\mathrm{C} \%$. Segundo Raij (1981), no caso de matéria orgânica estabilizada de solos bem drenados podese utilizar as relações 100:10:1:2 para C:N:S:P. Com essa base de dados gerou-se o mapa de potencial produtivo dos solos calculado através do modelo de estimativa de produtividade relativa.

Para analisar a distribuição das áreas de cana-de-açúcar no município de Piracicaba foram confeccionados outros mapas temáticos. Utilizou-se um modelo digital de elevação do terreno para calcular as declividades do relevo do município, um mapa de distâncias de usinas ás áreas com cana-de-açúcar, um mapa de uso do solo onde foram mapeadas as áreas 
ocupadas com cana-de-açúcar. Para confecção deste último foi utilizado imagem TM landsat bandas 3,4 e 5 de 1998. Todas essas informações constituem a base de dados de Giannotti (2001), que foi disponibilizada e usada neste trabalho. Para gerar os mapas foi utilizado o software TNT MIPS Micro Images, que é um programa de sistema de informações geográficas (SIG).

\subsection{Modelo FAO}

A FAO (Food and Agriculture Organization of the United Nations), considera que, em função da complexidade do sistema solo-planta-atmosfera, os conhecimentos acerca deste tema devem ser reduzidos para um número manejável de componentes possibilitando análises significativas do efeito da água sobre a cultura.

\subsubsection{Produtividade Potencial - Método da Zona Agroecológica}

A metodologia para calcular a produtividade potencial de uma cultura foi desenvolvida para servir como estimativa em base continental. Contudo o método pode ser utilizado em condições regionais ou localidades específicas.

Para determinado clima a produtividade potencial possível de uma cultura padrão é calculada aplicando-se o conceito preconizado por de Wit (1965) a partir de dados de radiação solar, fazendo-se posteriormente correções para a cultura. Neste modelo pressupõe-se que as necessidades hídricas, de fertilidade e fitossanitárias da cultura estejam sendo plenamente satisfeitas. A metodologia usada no presente trabalho, emprega adaptações propostas por Barbieri \& Tuon (1992), que considera as variações do índice de área foliar durante o ciclo, possibilitando os cálculos para cada período fenológico.

A produtividade potencial (PP, $\mathrm{kg} \mathrm{ha}^{-1}$ de matéria seca) da cultura é dada pelo somatório da produtividade potencial de cada subperíodo (p). esta por sua vez, é dada pela taxa de fotossíntese bruta $\left(\mathrm{Fb}, \mathrm{kg}\right.$ ha ${ }^{-1}$.dia de matéria 
seca) corrigida por fatores adimensionais relativos ao índice de área foliar (IAF) específico para a cultura $\left(C_{I A F}\right)$, processo de respiração $\left(C_{R}\right)$, á parte colhida $\left(\mathrm{C}_{\mathrm{C}}\right)$, multiplicando pelo número de dias correspondente a fase considerada (ND):

$$
P P=\sum_{p=1}^{n}\left(F b \cdot C_{I A F} \cdot C_{R} \cdot C_{C} \cdot N D\right)
$$

A fotossíntese bruta é calculada pela soma da que ocorre em períodos nublados (Fbn) e da que ocorre em períodos com céu sem nuvens (Fbc).

$$
\mathrm{Fb}=\mathrm{Fbn}+\mathrm{Fbc}
$$

Por sua vez, a Fbn é função da radiação solar no topo da atmosfera (Qo) corrigido por um fator de resposta fotossíntética à temperatura (cTn), relativo a cada grupo de cultura e razão de insolação $(\mathrm{n} / \mathrm{N})$ :

$$
\mathrm{Fbn}=(31,7+0,219 \times \text { Qo }) \times \mathrm{cTn} \times(1-\mathrm{n} / \mathrm{N})
$$

A Fbc pode ser interpretada de forma análoga àFbn, de scrito abaixo:

$$
\mathrm{Fbc}=(107,3+0,36 \times \mathrm{Qo}) \times \mathrm{Ctc} \times \mathrm{n} / \mathrm{N}
$$

Os fatores cTn e cTc são fatores de resposta fotossíntética da cultura em relação à temperatura em dia nublado e sem nuvens respectivamente. $O$ tipo de cultura define estes fatores. Para a cultura da cana-de-açúcar (Grupo IV, Planta C4) temos os seguintes fatores:

Para períodos com temperaturas $\geq 16,5^{\circ} \mathrm{C}$ :

$$
\begin{aligned}
& \text { CTn }=-1,064+0,173 \times \mathrm{T}-0,0029 \times \mathrm{T}^{2} \\
& \text { CTc }=-4,16+0,4325 \times \mathrm{T}-0,00725 \times \mathrm{T}^{2}
\end{aligned}
$$


Para períodos com temperaturas $\leq 16,5^{\circ} \mathrm{C}$ :

$$
\begin{aligned}
& \text { CTn }=-4,16+0,4325 \times \mathrm{T}-0,00725 \times \mathrm{T}^{2} \\
& \text { CTc }=-9,32+0,865 \times \mathrm{T}-0,0145 \times \mathrm{T}^{2}
\end{aligned}
$$

De Wit (1965) considerou uma cultura padrão com IAF 5. Para IAFs menores, em uma cultura específica, deve-se aplicar a correção proposta por Barbieri \& Tuon (1992).

$$
\begin{aligned}
& C_{\text {IAF }}=0,0186+0,37 \times I A F-0,035 \times I A F^{2} \\
& \text { se IAF }>5, C_{\text {IAF }}=1
\end{aligned}
$$

Durante a fotossíntese e crescimento da cultura, parte dos carboidratos são consumidos no processo de fotorespiração e outra parte na manutenção da matéria viva. Este consumo é fortemente dependente da temperatura, havendo uma relação direta entre temperatura e respiração. Assim a correção para respiração $\left(C_{R}\right)$ é dada por:

$$
\begin{array}{ll}
C_{R}=0,6 & \text { se } \leq T 20^{\circ} \mathrm{C} \\
C_{R}=0,5 & \text { se }>\text { T } 20^{\circ} \mathrm{C}
\end{array}
$$

Geralmente, apenas uma parte da metéria seca total é colhida, seja na forma de grãos, fibras, ou açúcar. A relação entre a produtividade obtida e a matéria seca total é dada pelo índice de colheita $\left(\mathrm{C}_{\mathrm{C}}\right)$, que no caso do presente estudo será considerado como igual a 0,25 (Barbieri \& Tuon, 1992).

O valor resultante da produtividae potencial (PP) da equação é expresso em Kg de matéria seca de colmo por hectare.

\subsubsection{Produtividade realizável}

Esse método foi descrito por Doorembos \& Kassan (1979) e é associado àFAO, sendo muitas vezes denominado método $F A O$ de estimativa de produtividade de culturas. Originalmente, a produtividade calculada pelo modelo é denominada como produtividade real. Essa denominação causa certa 
confusão uma vez que, na realidade, essa "produtividade real" é a estimativa da produtividade gerada pelo modelo. Assim, neste trabalho a produtividade real será designada como produtividade realizável.

Quando as necessidades hídricas da cultura são atendidas plenamente, a evapotranspiração real (Etr) é igual á evapotranspiração da cultura (Etc), não ocorrendo perdas de produtividade. Todavia, quando o suprimento de água é insuficiente, ocorre que Etr < Etc, ocasionando então uma perda de produtividade, proporcional a este déficit no suprimento hídrico.

A resposta de produtividade da cultura em relação ao suprimento de água é quantificada pelo fator de resposta da cultura (ky), que varia de acordo com as fases fenológicas (Tabela 2) e que relaciona a queda de rendimento relativo (1 - PR / PP) como déficit de evapotranspiração relativa representado por (1 - ETr / ETc) de acordo com a equação abaixo:

$1-\mathrm{PR} / \mathrm{PP}=\mathrm{ky} \cdot(1-$ Etr $/$ Etc $)$

onde:

$\mathrm{PR}=$ produtividade realizável

$\mathrm{PP}=$ produtividade potencial

Etr $=$ evapotranspiração real

Etc $=$ evapotranspiração da cultura

Utilizou-se o programa BHCULT de Rolim et al. (1998) para realização tanto do balanço hídrico como para o cálculo das produtividades potenciais e realizáveis pelo método de Doorembos \& Kassam (1979). Este programa realiza os cálculos com CAD e Ky variáveis em função das fases da cultura. Os parâmetros utilizados estão na Tabela 5. 
Tabela 5. Parâmetros utilizados como entrada para o modelo de Doorembos \& Kassam (1979) de estimativa de produtividade de culturas.

\begin{tabular}{lccccc}
\hline \multicolumn{1}{c}{ Fase } & Duração (dias) & $\mathrm{Kc}$ & $\mathrm{Ky}$ & $\mathrm{IAF}$ & $\mathrm{CAD}$ \\
\hline Estabelecimento da cultura & 60 & 0,4 & 0,05 & 1,5 & 30 \\
Desenvolvimento vegetativo & 210 & 0,85 & 0,75 & 4,5 & 80 \\
Florescimento & 10 & 1,2 & 1 & 4,5 & 100 \\
Frutificação & 40 & 1,3 & 0,6 & 4 & 100 \\
Maturação & 40 & 0,5 & 0,1 & 3 & 100 \\
\hline
\end{tabular}

Os valores de Kc e Ky de Doorembos \& Kassan (1979), os de IAF foram obtido de Machado (1987). Utilizou-se umidade (U\%) de 70\% e coeficiente de colheita de 0,7 conforme Pereira et al. (2002). A temperatura basal foi considerada como $20^{\circ} \mathrm{C}$ (Magalhães, 1987). As fases de florescimento e frutificação estão aqui descritas embora não sejam realizadas pela cana-deaçúcar. O programa precisa realizar estas fases para completar o ciclo da cultura em questão. Foi considerado que o florescimento e frutificação fazem parte do desenvolvimento vegetativo, e estas três fases compôe a fase de formação da produção. 


\section{RESULTADOS E DISCUSSÃO}

\subsection{Testes do modelo em diferentes escalas}

\subsubsection{Teste com dados experimentais}

Para avaliar a aplicabilidade do modelo proposto realizou-se uma avaliação preliminar com resultados experimentais de Dias et al. (1999) e Maule et al. (2001). Os autores estudaram, entre outros fatores, as relações entre solos e produtividade de cana-de-açúcar em diferentes solos e regiões no Estado de São Paulo. Esta avaliação inicial foi importante uma vez que possibilitou testar a metodologia com resultados experimentais realizados em condições de maior controle e em uma escala maior.

Apesar de os experimentos terem sido conduzidos em localidades diferentes considerou-se que o clima não limitou a produtividade pois possibilitou a condução do experimento de forma adequada produzindo resultados consistentes. Os resultados dos experimentos e das produtividades estimadas estão na Tabela 6. 
Tabela 6. Relação entre a produtividade medida, obtida em experimentos conduzidos no Estado de São Paulo, e a produtividade estimada pelo modelo de estimativa de produtividade relativa.

\begin{tabular}{|c|c|c|c|c|c|c|}
\hline \multirow[b]{2}{*}{ Autor } & \multirow[b]{2}{*}{ Local } & \multirow[b]{2}{*}{ Variedade } & \multirow[b]{2}{*}{ Solo } & \multicolumn{3}{|c|}{ Produtividade $\left(\right.$ t.ha $^{-1}$ ) } \\
\hline & & & & Estimada ** & Medida relativa & Medida \\
\hline \multirow[t]{6}{*}{ Dias et al. (1999) } & Valparaiso & RB835486 & PVe $a b a / m$ & 84,42 & 72,51 & 144,31 \\
\hline & Valparaiso & RB72454 & $\mathrm{PVI}$ a a/m & 72,75 & 47,87 & 95,27 \\
\hline & Estrela D'Oeste & RB835089 & LV d m & 78,68 & 54,34 & 108,15 \\
\hline & Clementina & SP79-1011 & $\mathrm{PVe} a / \mathrm{m}$ & 74,35 & 55,71 & 110,87 \\
\hline & Araçatuba & SP81-2590 & PVe $a / m$ & 92,40 & 86,04 & 171,22 \\
\hline & Araçatuba & RB845210 & $\mathrm{AQ}$ a $a / m$ & 70,00 & 42,63 & 84,84 \\
\hline \multirow[t]{4}{*}{ Maule et al. (2001) } & Castilho & RB845257 & $\mathrm{PV} a / m$ & 100,00 & 100,00 & 199,00 \\
\hline & Castilho & RB835019 & $\mathrm{PV} a / \mathrm{m}$ & 78,67 & 61,80 & 123,00 \\
\hline & Castilho & RB845257 & $\mathrm{Pl} \mathrm{a} / \mathrm{m}$ & 99,58 & 83,41 & 166,00 \\
\hline & Castilho & RB845257 & $\mathrm{PI} \mathrm{a} / \mathrm{m}$ & 96,54 & 79,89 & 159,00 \\
\hline
\end{tabular}

Realizou-se uma análise de regressão que mostrou uma relação altamente significativa $(P<0,001)$ entre a produtividade estimada pelo modelo e a obtida nos experimentos considerados, com um coeficiente de correlação $r^{2}=0,91^{* *}$, mostrando que o modelo simula de forma adequada a produtividade da cana-de-açúcar (Figura 1).

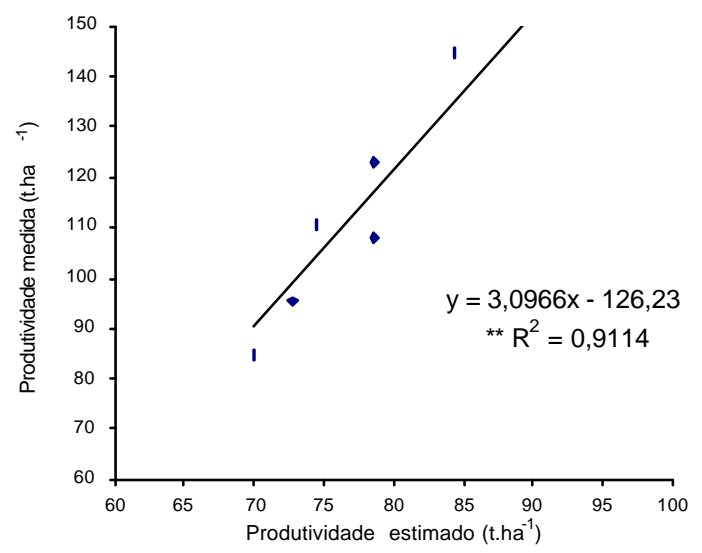

Figura 1 - Relação entre a produtividade relativa estimada pelo modelo de estimativa de produtividade relativa e a produtividade medida, obtida nos experimentos de Dias et al. (1999) e Maule et al. (2001). 


\subsubsection{Teste com dados de campo da Usina Santa Rita}

Foi utilizado o modelo de estimativa de produtividade relativa de cana-de-açúcar com base em parâmetros do solo em 5 variedades, a RB85113, SP80-1816, RB855536, RB835486 e a SP80-1842 que são representativas da Usina, com dados de campo da Usina Santa Rita, localizado no município de Santa Rita do Passa Quatro no Estado de São Paulo.

Os resultados mostraram uma relação estreita entre a produtividade relativa estimada e medida em cada variedade, exceto para a variedade SP801842, que apresentou pequena variação em produtividade entre os solos contrastantes. A análise de regressão apresentou $\mathrm{r}^{2}=0,59^{*}$, significativo a $5 \%$ (Figura 2).

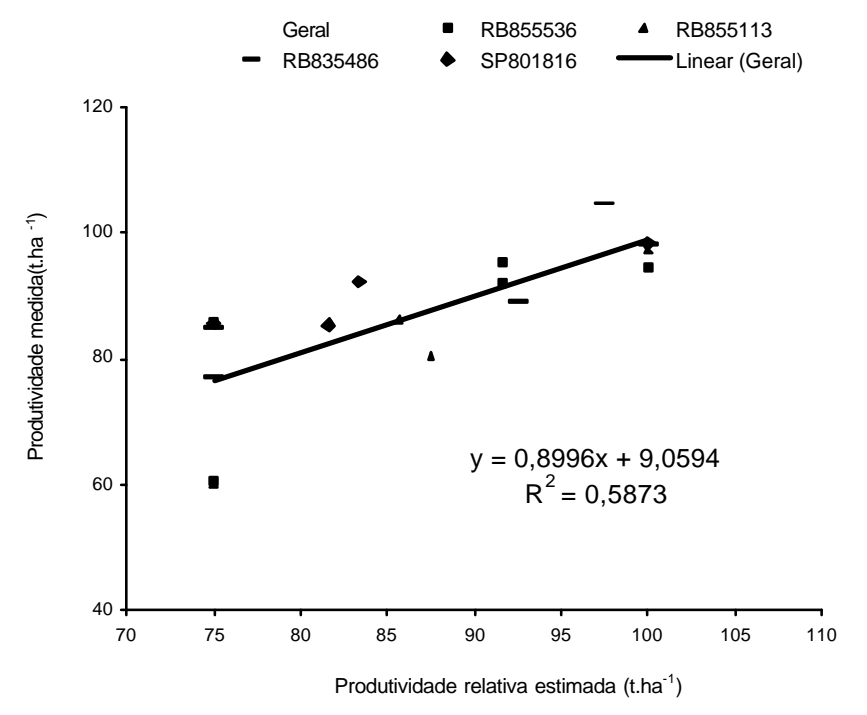

Figura 2 - Relação entre a produtividade relativa estimada pelo modelo de estimativa de produtividade relativa e a produtividade medida de 5 variedades na Usina Santa Rita 
Nota-se que apesar da existência de um critério para obtenção dos dados é possível que a natureza não experimental tenha agregado a estes causas de variação dos quais não se teve controle, causando grande variabilidade refletindo na perda de precisão do modelo em comparação com o seu desempenho nos dados experimentais.

\subsubsection{Teste com dados de campo da Usina Costa Pinto}

O modelo foi utilizado num ambiente operacional maior, do porte de uma grande fazenda ou empresa, entretanto numa escala de menor detalhe. Utilizou-se este modelo com dados obtidos também da rotina operacional da Usina Costa Pinto. A organização administrativa das áreas com cana-deaçúcar, nesta usina, é composta pelas zonas, que é o conjunto de fazendas que por as vez é formada pelos talhões.

As relações entre os resultados simulados $\mathrm{e}$ as produtividades obtidas em campo estão apresentados na Figura 3.
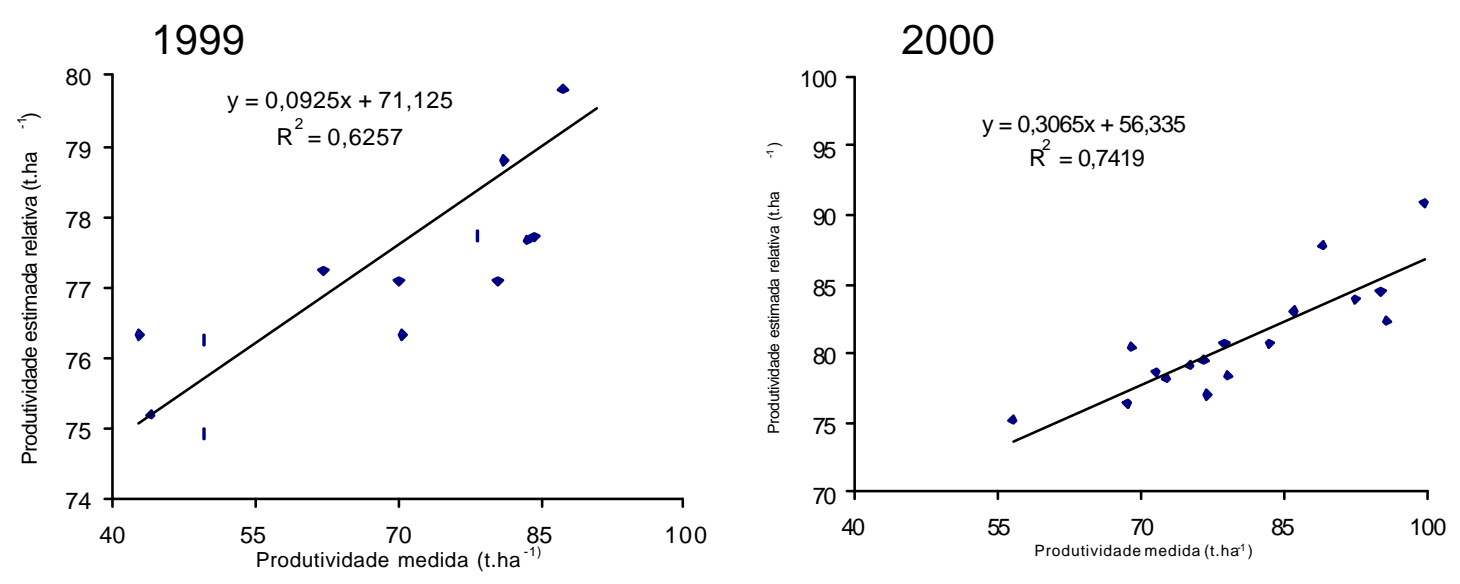

Figura 3 - Comparação entre produtividade relativa estimada pelo modelo e a produtividade medida em campo. As análises de variância de 1999 e 2000 foram significativas a $1 \%$. 
Nesta escala, em função da variabilidade inerente à dados de campo, trabalhou-se com dados médios a nível de fazenda por considerar-se que esta é uma unidade administrativa que tem características relativamente homogêneas de meio físico (solos e relevo), de clima e de manejo da cultura, tornando essa escala de trabalho adequada para o propósito deste estudo.

Neste caso o modelo simulou de forma satisfatória as produtividades relativas em comparação com os valores de produtividade obtida em campo. Mesmo trabalhando em nível de fazenda houve perda de precisão do modelo, mas continua trabalhando nos níveis de correlação obtidos no trabalho realizado na usina Santa Rita.

\subsubsection{Causas da variação dos dados de produtividade obtidos}

Observou-se que o modelo perde precisão com a redução da escala de trabalho, ou seja num escala de menor detalhe. Numa escala de maior detalhe, num ambiente de maior controle das causas de variação, o modelo apresentou boa precisão, tais condições são encontradas em experimentos conduzidos com esse propósito. Ao se trabalhar em uma escala de menor detalhe, onde o controle das variáveis é difícil, existe uma tendência de perda de precisão do modelo. Essa situação ocorre, por exemplo, no ambiente de uma empresa, onde o ritmo operacional impede ou não proporciona um controle comparável ao de um experimento.

Vários fatores contribuem para este fato, como por exemplo erro nas áreas de talhões, identificação incorreta do talhão, identificação incorreta dos caminhões que fazem o carregamento, carregamento de cana de mais de um talhão no mesmo caminhão, junção de talhões na ocasião do corte, incêndio, perda excessiva de cana no campo, dentre outros fatores. Para efeito de controle administrativo esses desvios não acarretam grande problema para usina, entretanto são suficientes para serem identificados numa análise criteriosa de avaliação de um modelo. A equipe técnica da usina tem 
consciência de que esse processo de coleta de informações é importante para diagnósticos mais precisos e para atividades de planejamento.

Em trabalhos futuros, dessa natureza, a coleta de dados de campo deve ser acompanhada para garantir maior precisão dos resultados dos modelos viabilizando melhor aplicabilidade destes.

\subsection{Potencial produtivo teórico das classes de solos avaliada através da produtividade medida da área de estudo}

O potencial de produção dos solos é basicamente função de suas propriedades químicas, físico-hídricas e da paisagem local. Uma forma de estimar esse potencial teórico é através de uma contabilização da produção de cana-de-açúcar nos diferentes solos de uma região.

Através dos mapas de localização dos talhões da Usina e da carta pedológica semi-detalhada da quadrícula de Piracicaba, escala 1:100.000 (Oliveira \& Prado, 1989), determinou-se a classe de solo aproximada das fazendas. Em função da escala do mapa de solos considerou-se apenas o nível de classe de solo. As classes predominantes encontradas foram latossolos, argissolos e neosolos litólicos. Os neosolos litólicos, por definição, não apresentam horizonte $B$, entretanto as análises de terra sempre apresentam duas profundidades, $0-25 \mathrm{~cm}$ e $25-50 \mathrm{~cm}$, assim assumiu-se que os neosolo litólicos considerados apresentavam pelo menos $50 \mathrm{~cm}$ de profundidade.

Os dados obtidos foram agrupados em relação a classificação dos solos para se obter a produtividade média de cana para cada classe considerada. Com isso procurou-se estabelecer uma indicativo do potencial produtivo dessas classes de solo através dos rendimentos da cana-de-açúcar em diferentes anos. Para o ano de 1999 o número de talhões utilizados para representar o latossolo, argissolo e neossolo litólico foram 23, 231 e 51 respectivamente. Em 2000 foram utilizados 15, 92 e 15 talhões respectivamente para latossolo, argissolo e neossolo litólico. Finalmente em 2001 foram 
utilizados 17, 40 e 17 talhões respectivamente para latossolo, argissolo e neossolo litólico.

Verificou-se que existe uma tendência na qual a classe dos latossolos apresentam maior potencial relativo de produção, seguido pelos argissolos e pelos neosolos litólicos. Os rendimentos médios dos três anos foram de 89,0 t.ha ${ }^{-1}, 75,4$ t.ha ${ }^{-1}$ e 70,7 t.ha ${ }^{-1}$ para os latossolos, argissolos e neosolos litólicos respectivamente. Foram considerados todos os cortes, com exceção da cana planta, e todas as variedades, em cada ano (Figura 4).

Os latossolos constituem-se numa importante classe de solo em razão de sua distribuição nas áreas canavieiras do Estado de São Paulo e também pelo seu alto potencial produtivo (Kofler \& Donzeli, 1987; Penati, 1991). Dias et al. (1999) e Maule et al. (2001) observaram maior potencial relativo dos solos argissolos, atribuindo essa importância æ̀ suas propriedades físicas, em especial à relação textural, que tem efeito direto no armazenamento de água e no tempo de residência desta no perfil do solo.

Os neosolo litólicos apresentaram o menor potencial relativo observado. Estes apesar de apresentarem boas condições de fertilidade, apresentam limitações inerentes à suas características morfológicas. São solos rasos, que geralmente apresentam impedimentos ao desenvolvimento radicular. Além disso, o regime hídrico destes solos é ústico, ou seja o perfil do solo está seco na maior parte do ano, caracterizando-se também numa importante restrição ao desenvolvimento vegetal. 


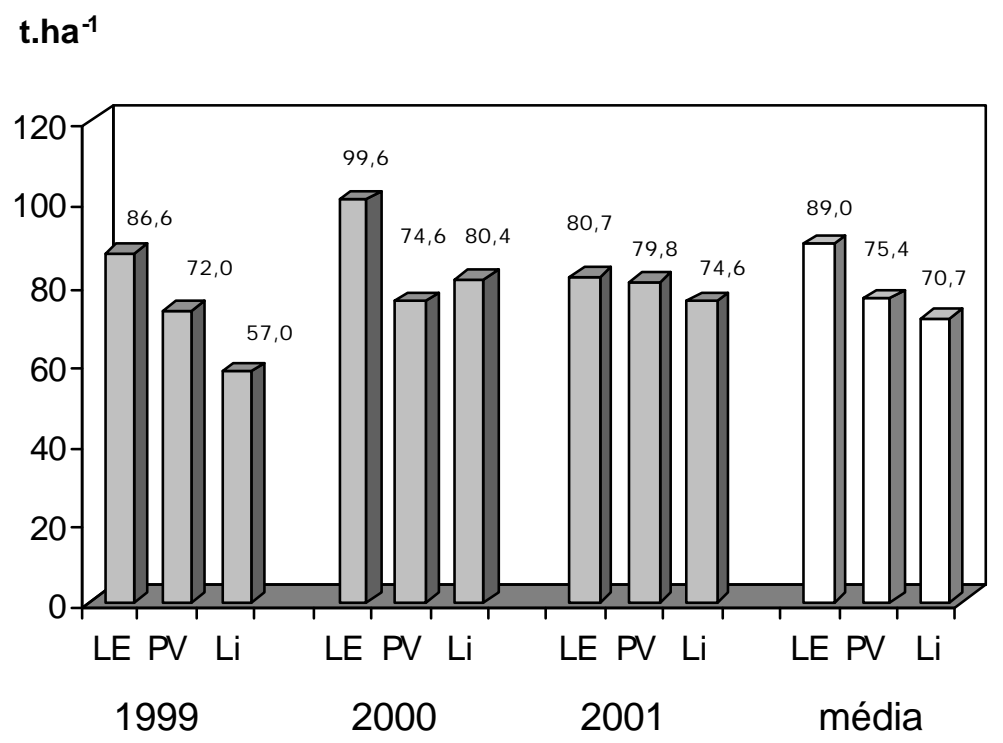

Figura 4 - Produtividade média de cana-de-açúcar (t.ha-1 ${ }^{-1}$ obtida no campo em diferentes classes de solos nos anos de 1999, 2000 e 2001 na usina Costa Pinto e a média geral por classe de solo.

\subsection{Potencial produtivo teórico das classes de solos avaliada através do modelo para estimativa da produtividade relativa baseado em parâmetros do solo}

A metodologia utilizada neste trabalho para avaliação do potencial produtivo dos solos baseia-se nos teores de P, K, T, V\% e teor de argila. Com isso obtém-se um resultado ponderado que representa o status relativo da fertilidade do solo, de forma que o conjunto desses parâmetros tem importância maior que se fossem considerados isoladamente, conforme o conceito de produtividade (Raij, 1981).

A Figura 5 apresenta as pontuações das diferentes classes de solos. A média das pontuações dos três anos foram de 19,3, 12,3 e 17,4 para os latossolos, argissolos e neosolos litólicos respectivamente. Nota-se que a pontuação dos neosolos litólicos é maior nos anos de 1999 e 2001, indicando que este solo possui alto potencial quanto a suas características químicas. 
Entretanto os argissolos apresentaram rendimento superior aos neosolos litólicos nos anos de 1999 e 2001 (Figura 5). Todavia o comportamento se inverte no ano de 2000. O argissolo tem produtividade inferior ao neossolo litólico apesar deste último possuir uma pontuação menor que o argissolo.

Isso mostra que outras características do solo, fatores externos ou ainda as interações entre eles podem ser mais determinantes que somente as características químicas e o teor de argila. Por exemplo, as características morfológicas, físicas e da paisagem em que ocorrem os neosolos litólicos foram limitantes a ponto de impedir que sua fertilidade teórica se expressasse em maior rendimento. Já os latossolos obtiveram as maiores pontuações em 1999 e 2000 confirmando seu potencial para a cana-de-açúcar, como verificado na Figura 4.

Deve-se considerar que este status, que representa o potencial teórico neste trabalho, está baseado fortemente nas propriedades químicas dos solos, sem no entanto considerar aspectos morfológicos ou outros inerentes a classificação de cada solo, que porventura podem vir a se tornar mais importantes ou limitantes em algumas situações. Tal fato evidencia que é importante considerar outros aspectos relacionados ao solo, além da fertilidade, na elaboração de modelos que relacionem parâmetros do solo e produtividade.

Assim, em futuros desenvolvimentos do modelo estas variáveis adicionais devem ser consideradas para o aprimoramento do mesmo. 


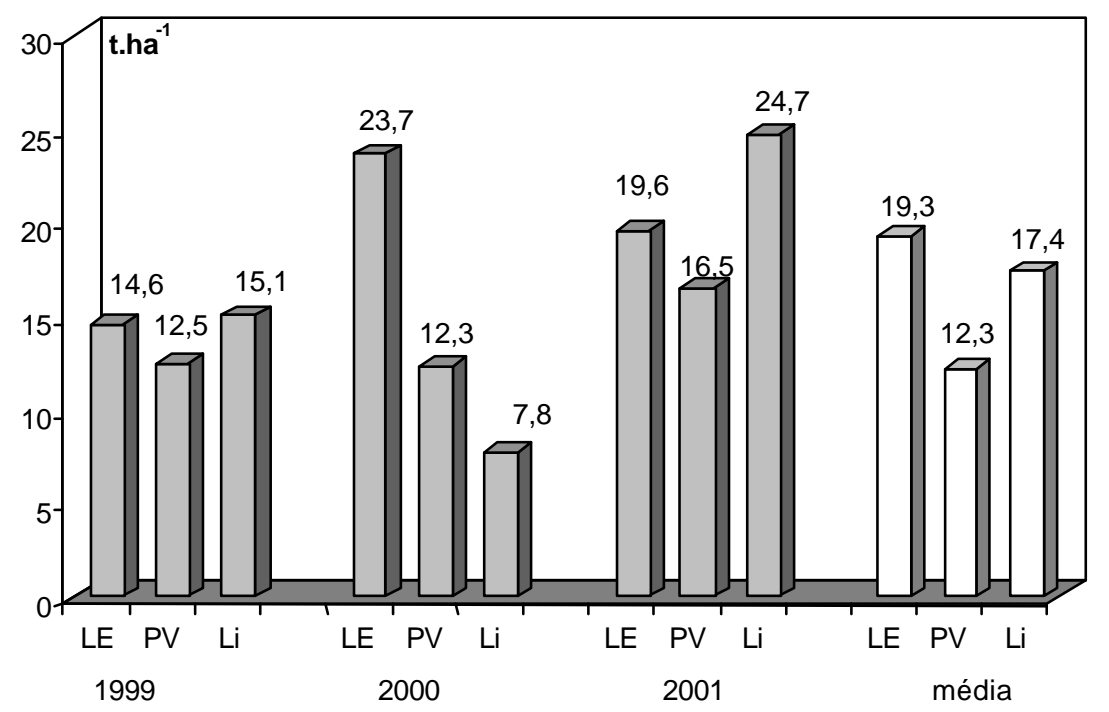

Figura 5 - Pontuação média de cana-de-açúcar (t.ha $\left.{ }^{-1}\right)$ das classes de solos nos anos de 1999, 2000 e 2001 na usina Costa Pinto e a média geral por classe de solo.

Numa análise global, a relação entre pontuação e produtividade mostrou-se relativa, e nem sempre um bom status de fertilidade resulta em alta produtividade, como foi verificado para o neossolo litólico. De qualquer maneira, o modelo atual é suficientemente preciso para estimar a produtividade relativa das áreas de canavial.

\subsection{Fatores que influenciaram a produtividade da cana-de-açúcar obtidos através de análise de regressão linear múltipla com seleção de variáveis pelo método stepwise}

Para se conhecer as variáveis que influenciaram a produtividade da cana-de-açúcar nos diferentes anos foi realizada uma análise de regressão linear múltipla com seleção de variáveis pelo método stepwise. Para isso utilizou-se o software SAS "Statistical Analysis Systen" versão 6.11 (SAS Institute, 1989). A análise de variância foi testada com nível de significância de 15\% de probabilidade (Beauclair, 1994, Scarpari, 2002). 
Foram consideradas as variáveis: numero do corte, variedade e os resultados analíticos da análise de terra, a saber; $\mathrm{pH}$, matéria orgânica (M.O.), fósforo $(\mathrm{P})$, potássio $(\mathrm{K})$, cálcio $\left(\mathrm{Ca}^{+2}\right)$, magnésio $\left(\mathrm{Mg}^{+2}\right)$, alumínio $\left(\mathrm{Al}^{+3}\right)$, hidrogênio + alumínio $(\mathrm{H}+\mathrm{Al})$, capacidade de troca de cátions (CTC), saturação por bases $(\mathrm{V} \%)$, saturação por alumínio $(\mathrm{m} \%), \mathrm{K}_{2} \mathrm{O}$ e $\mathrm{P}_{2} \mathrm{O}_{5}$, estes dois últimos representam as adubações de potássio e de fósforo. A soma de bases não foi incluída por ser colinear com as bases $\mathrm{K}, \mathrm{Ca}^{+2} \mathrm{e} \mathrm{Mg}^{+2}$. As análises fram realizadas para os anos considerados e para as camadas de $0-25 \mathrm{~cm}$ e 25 a $50 \mathrm{~cm}$ separadamente.

Esta análise de regressão linear múltipla foi realizada tendo como variáveis base o segundo corte, em razão de não estar sendo utilizado o primeiro corte, e a variedade RB72-454 por esta ser considerada uma variedade entre as mais produtivas na usina e por ser a mais freqüente nas áreas plantadas da mesma.

È importante salientar que esta análise de regressão representa especificamente os fatores que interferiram $\mathrm{n}$ a produtividade nos anos considerados e com o estado de nutrientes nos solos representados pelas análises destes mesmos anos. As interferências do clima, pragas, doenças e outros fatores foram considerados como erros não mensuráveis. 
Tabela 7 - Resultado da análise de regressão linear múltipla com seleção de variáveis, método stepwise, realizada entre as variáveis de análise de solo das camadas de $0-25 \mathrm{~cm}$ e $25-50 \mathrm{~cm}$, número de cortes e variedades no ano de 1999.

\begin{tabular}{clccc}
\hline $\begin{array}{c}\text { Camada } \\
(\mathbf{c m})\end{array}$ & Variável & $\begin{array}{c}\text { Parâmetro } \\
\text { Estimado }\end{array}$ & $\begin{array}{l}\text { Desvio } \\
\text { Padrão }\end{array}$ & Pr $>\mathbf{F}^{\star}$ \\
\hline & Intercept & 80,4254 & 4,7439 & $<, 0001$ \\
& RB76-5418 & $-17,7448$ & 4,0209 & $<, 0001$ \\
& RB78-5148 & $-25,1307$ & 5,6736 & $<, 0001$ \\
$\mathbf{0 - 2 5}$ & SP70-1143 & $-26,3440$ & 9,2137 & 0,0049 \\
& SP80-1842 & 23,2315 & 7,9451 & 0,0041 \\
& 5o corte & $-10,7296$ & 3,6218 & 0,0036 \\
& Ca & 0,6366 & 0,1942 & 0,0013 \\
& Mg & $-1,0878$ & 0,4440 & 0,0156 \\
& CTC & $-0,0503$ & 0,0335 & 0,1363 \\
& & & & \\
& Intercept & 10,8359 & 20,9025 & 0,6050 \\
& RB76-5418 & $-23,0907$ & 4,2931 & $<, 0001$ \\
& RB78-5148 & $-33,6662$ & 5,0302 & $<, 0001$ \\
& SP70-1143 & $-27,2444$ & 9,3696 & 0,0043 \\
& SP80-1842 & 18,4284 & 8,1280 & 0,0250 \\
& 5o corte & $-11,3716$ & 3,7749 & 0,0031 \\
& PH & 14,8523 & 4,2850 & 0,0007 \\
& H + Al & 0,1912 & 0,0880 & 0,0315 \\
\hline
\end{tabular}


Tabela 8 - Resultado da análise de regressão linear múltipla com seleção de variáveis, método stepwise, realizada entre as variáveis de análise de solo das camadas de $0-25 \mathrm{~cm}$ e $25-50 \mathrm{~cm}$, número de cortes e variedades no ano de 2000 .

\begin{tabular}{clccc}
\hline $\begin{array}{c}\text { Camada } \\
(\mathbf{c m})\end{array}$ & Variável & $\begin{array}{c}\text { Parâmetro } \\
\text { Estimado }\end{array}$ & $\begin{array}{c}\text { Desvio } \\
\text { Padrão }\end{array}$ & Pr $>\mathbf{F}^{\star}$ \\
\hline & Intercept & 90,1797 & 7,1053 & $<, 0001$ \\
& 4o corte & 10,7760 & 3,9859 & 0,0076 \\
& MO & 0,8914 & 0,2994 & 0,0034 \\
$\mathbf{0 - 2 5}$ & Mg & $-1,1544$ & 0,2238 & $<, 0001$ \\
& Al & $-0,4113$ & 0,1833 & 0,0263 \\
& K2O & $-0,2020$ & 0,0342 & $<, 0001$ \\
& & & & \\
& Intercept & 85,1374 & 6,9854 & $<, 0001$ \\
& RB85-5156 & 9,4445 & 6,1773 & 0,1284 \\
& 4o corte & 8,7158 & 3,8480 & 0,0249 \\
& 60 corte & $-11,6810$ & 7,8994 & 0,1413 \\
& MO & 1,5338 & 0,3432 & $<, 0001$ \\
& Mg & $-0,7832$ & 0,2371 & 0,0012 \\
& H + Al & $-0,1447$ & 0,0560 & 0,0107 \\
& K2O & $-0,1864$ & 0,0346 & $<, 0001$ \\
\hline
\end{tabular}


As Tabelas 7 e 8 mostram que existem muitos fatores que interferiram na produtividade da cana-de-açúcar nas colheitas de 1999 e 2000. De modo geral contatou-se, mais claramente, o efeito das diferentes variedades e dos diferente cortes $\mathrm{n}$ a produtividade, apesar de existirem variações entre as variedades e os cortes em relação aos anos. A influência destes dois fatores é esperada uma vez que são fatores que sabidamente têm influencia na produtividade da cana-de-açúcar (Paranhos, 1987). A relação entre as variedades, a produtividade e o número do corte e mostraram-se significativas no mínimo a 5\%.

As variedades que influenciaram a produtividade geralmente 0 fizeram de forma negativa, depreciando a produtividade em comparação com a variedade RB72-454, considerada variável base, demonstrando que esta provavelmente é a variedade de melhor desempenho para a região de Piracicaba.

As variedades que apresentaram relação significativa com a produtividade foram a RB76-5418, RB78-5148, SP70-1143, SP80-1842, RB855156, RB85-5113, RB85-5536. Destas a SP80-1842, RB85-5156 e a RB855113 mostraram acréscimo relativo de rendimento em pelo menos um ano.

$\mathrm{O}$ número de cortes também mostrou influência negativa $\mathrm{n}$ a produtividade, depreciando-a. O que também é uma constatação esperada, conforme já descrito por Nunes Júnior (1987).

Com relação aos atributos químicos do solo verificou-se ausência de uma tendência ou uma variável principal com evidente influência $n$ a produtividade nos diferentes anos. As variáveis que apresentaram relação significativa a pelo menos $15 \%$, com a produtividade foram, $\mathrm{Ca}, \mathrm{Mg}, \mathrm{T}, \mathrm{pH}$, $\mathrm{H}+\mathrm{Al}$, M.O., Al, $\mathrm{K}_{2} \mathrm{O}$, V\% e M\%.

O Mg apresentou correlação significativa a $5 \%$ na camada de $0-25 \mathrm{~cm}$ no ano de 1999, sendo que no ano de 2000 apresentou correlação significativa a $1 \%$ na camada de $0-25 \mathrm{~cm}$ e a $5 \%$ na de $25-50 \mathrm{~cm}$ em 2000 , mas seu efeito foi 
depreciativo. Uma das razões para esse fato pode ser atribuída a ocorrência de relações $\mathrm{Ca} / \mathrm{Mg}$ com valores abaixo do desejável. Assim, altos teores de Mg no solo, se por um lado aumentam sua disponibilidade, pode também estar provocando menor absorção de $\mathrm{Ca}$ e $\mathrm{K}$ pela planta, depreciando a produtividade da cana-de-açúcar. Lopes (1998) relata que em condições de alta relação $\mathrm{Ca} / \mathrm{Mg}$ as plantas podem absorver menos $\mathrm{Mg}$ e a adubação potássica pode diminuir a absorção de Mg por gramíneas.

Outra variável de comportamento contraditório foi V\% que em 2001 apresentou correlação significativa a 1\% depreciando a produtividade. A M.O. do solo se destacou como variável que contribuiu com acréscimo de rendimento.

Bittencourt et al. (1989) relata que em alguns casos em talhões comerciais de cana-de-açúcar, os dados das análises de solo não explicam e não prevêem as diferentes classes de produtividade. $O$ autor comenta que as análises podem não estar quantificando os teores realmente disponíveis no solo.

O fato de diferentes variáveis estarem influenciando a produtividade da cana-de-açúcar reforça a complexidade das interações entre os fatores que compõe a produtividade. Isso explica a variabilidade dos dados obtidos em campo. Além disso evidencia a necessidade de direcionar as linhas de pesquisa para uma abordagem integradora, que estude os fatores de forma não estanque com o objetivo de aprimorar modelos que gerem resultados onde as diferentes ciências tenham sido ponderadas.

È interessante notar que os parâmetros utilizados no modelo baseado em parâmetros de solo, $\mathrm{P}, \mathrm{K}, \mathrm{T}, \mathrm{V} \%$ e argila, não foram necessariamente variáveis que influenciaram a produtividade de forma determinante. Dentre os parâmetros utilizados no modelo, o K, T, e V\% apresentaram relações significativas com a produtividade em pelo menos um ano, sendo que $T$ teve relação significativa apenas a $15 \%$ e os demais parâmetros a 5\%. A variável $P$ não apresentou relação significativa em nenhum 
ano. Infere-se que as adubações suprem a planta mas não alteram a fertilidade, Assim a produtividade da cana-de-açúcar é superior aquela esperada e pela fertilidade do solo.

Esse fato demonstra que as ferramentas estatísticas, se utilizadas de forma isolada, podem não ser a melhor metodologia para elaborar modelos, nem para identificar relações de causa dos fatores complementares e concorrentes com seu efeito na produtividade. No presente trabalho o modelo para estimativa da produtividade relativa com base em parâmetros do solo proposto não traz nenhum argumento estatístico embutido em sua concepção, mas está baseado em alicerces teóricos sólidos, demonstrando ser adequado e eficiente na estimativa de produtividade da cana-de-açúcar.

\subsection{Aplicação prática do modelo para estimativa da produtividade relativa com base em parâmetros do solo}

Uma das possibilidades de utilização deste modelo é como ferramenta de diagnóstico e de planejamento regional. $\mathrm{O}$ usuário pode ser uma empresa de grande porte ou mesmo os governos municipais, estaduais, federais e outros órgãos de atuação no setor.

Neste estudo o modelo foi aplicado no município de Piracicaba. O objetivo foi tentar verificar como a cana-de-açúcar se distribui geograficamente em relação a aspectos do meio físico do município, como é o potencial produtivo da cana-de-açúcar em relação aos solos do município, quais são os fatores que determinam a ocupação das terras com cana-de-açúcar no município e como as usinas interagem com esse cenário. As variáveis utilizadas foram a distância das áreas do município e das áreas de cana-de-açúcar em relação às usinas, a declividade do relevo e o potencial produtivo dos solos estimado através do modelo de solos. As relações entre as variáveis e a interpretação destas foi realizada através de histogramas de distribuição de freqüência dessas variáveis. 


\subsubsection{Distância das usinas em relação às áreas com cana-de-açúcar e às áreas do município}

Para avaliar a distribuição das áreas de cana-de-açúcar no município com relação àdistância de usinas foram consideradas 4 usinas de grande porte da região de Piracicaba, a Usina Costa Pinto, Usina Furlan, Usina Santa Helena e Usina Iracema, esta última localizada no município vizinho de Iracemápolis porém com muitas áreas no município de Piracicaba.

A Figura 6, representa a distância das usinas em relação somente ás áreas com cana-de-açúcar e em relação àárea total do município, ocupada ou não com cana-de-açúcar. Nota-se que existe rítida tendência de preferência das usinas em ocupar as áreas próximas a elas com a cultura da cana-deaçúcar. Verifica-se $50 \%$ das áreas somente com cana-de-açúcar estão a menos de $12 \mathrm{~km}$ de uma usina e $50 \%$ das áreas do município estão a menos de $17 \mathrm{~km}$ de uma usina. As áreas ocupadas somente com cana-de-açúcar apresentam $7,5 \%$ do total das áreas com cana-de-açúcar ocupam a classe de áreas que estão a aproximadamente $10 \mathrm{~km}$ de uma usina. A partir de aproximadamente $15 \mathrm{~km}$ de distância de uma usina as áreas somente com cana-de-açúcar passam a ser menos freqüentes. Alguns picos de freqüência em torno de $25 \mathrm{a}$ $30 \mathrm{~km}$ podem ser áreas de fornecedores de cana-de-açúcar. O mapa de distâncias das áreas com cana-de-açúcar e do município em relação às usinas está no Anexo 1. 


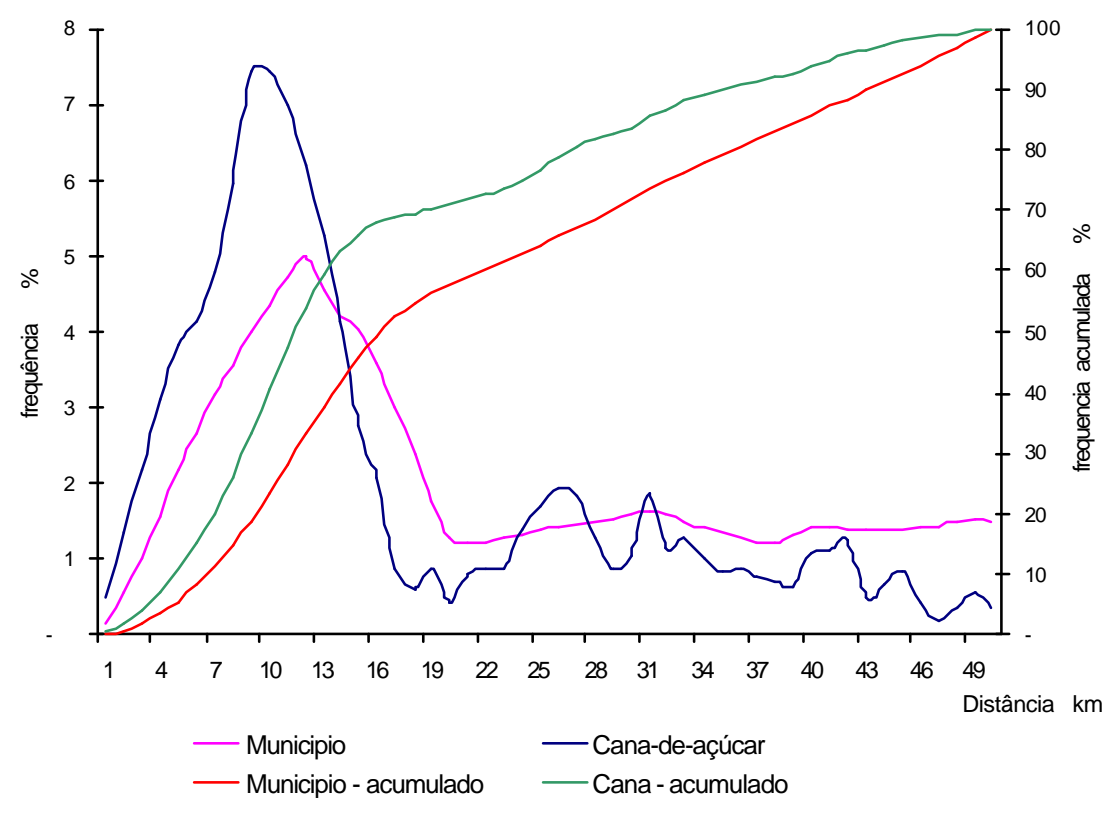

Figura 6 - Histograma de distribuição de freqüência da distância das áreas com cana-de-açúcar e das áreas do município em relação às usinas.

Com esta informação conclui-se que um critérios importante para tomada de decisão quanto à imp lantação de canavial é a distância em relação æ̀ usinas. Elas preferem áreas próximas à solos melhores. Essa decisão está ligada ao custo de transporte da cana-de-açúcar até á usina. Este cenário entretanto estará sujeito a modificações tendo em vista que o crescimento urbano, a restrição da queima prévia da cana-de-açúcar à colheita e a conseqüente tendência de mecanização da colheita são fatos que alterarão o cenário de ocupação as terras do município. Para onde a cana-de-açúcar irá é uma questão que modelos como o utilizado neste trabalho podem ajudar a responder. 


\subsubsection{Ocorrência das áreas com cana-de-açúcar e das áreas do município em relação aos solos e seu potencial produtivo}

A Figura 7 representa a distribuição geográfica dos potenciais produtivos dos solos do município e a freqüência com que as áreas somente com cana-de-açúcar e as áreas do município as ocupam. Em média tanto as áreas de cana-de-açúcar como as áreas do município estão em solos com potencial de produção de 91,8 .ha $^{-1}$.

A maior freqüência de áreas com cana-de-açúcar no município está em solos com potencial entre 90 e 92t.ha ${ }^{-1}$. Nota-se que na classe de solos com potencial produtivo de $91{\mathrm{t} . h a^{-1}}^{\circ}$ município apresenta áreas que podem não estar sendo ocupadas com cana-de-açúcar. Estas representam áreas de expansão potencial. Mas é nítida a predominância com que a usina ocupa solos de alto potencial. $50 \%$ das áreas com cana-de-açúcar estão em solos com potencial acima de 91 t.ha ${ }^{-1}$ e na classe de 96 tha $^{-1}$ verifica-se uma salto de ocorrência de áreas com somente cana-de-açúcar.

È interessante notar que as usinas não excluem as áreas de menor potencial, mas essas são menos freqüentes em comparação com a ocorrência em melhores solos. Ranieri \& Sparovek (1993) e Sparovek \& Lepsch (1995) observaram que a cana-de-açúcar avançou em áreas ocupadas por pastagem no período de 1962 a 1991.

Esse fato tem relação com a preferência das usinas por áreas próximas à elas, fazendo com que as mesmas ocupem áreas com baixo potencial. Tal fato é preocupante uma vez que esse critério de ocupação provavelmente não contempla a sustentabilidade do solo e do ambiente, esse fato foi considerado por Sparovek \& Lepsch (1995). Por outro lado Levy \& Sparovek (1995) sugerem que a redistribuirão das áreas com cana-de-açúcar podem contribuir para uma ocupação de áreas de forma adequada com a capacidade de uso das terras. 


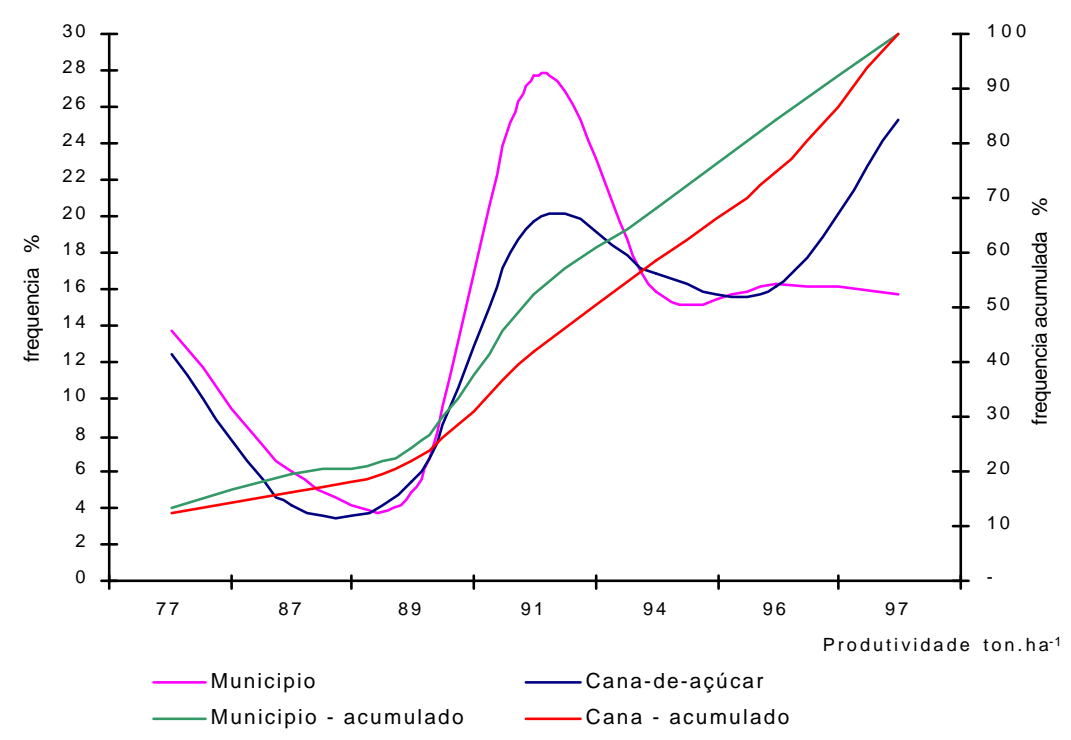

Figura 7 - Histograma de distribuição de freqüência das áreas com cana-deaçúcar em relação ao potencial produtivo dos solos do município de Piracicaba

Provavelmente o critério inicial para escolha de área para instalação das usinas baseou-se nos solos. As usinas procuraram instalar as usinas em solos considerados bons para a cultura. Com o crescimento do números de usinas instaladas e a expansão das áreas cultivadas com cana-de-açúcar no município, as usinas optaram por instalar canaviais próximos a elas independentemente de ocupar ou não áreas apropriadas ao seu cultivo. Isso foi verificado por Muramoto et al. (1993). Assim solos com baixo potencial foram ocupados com cana-de-açúcar em função de sua proximidade com a usina, mas apesar disso no cômputo geral do município as áreas ocupadas com canade-açúcar estão em solos com alto potencial produtivo. O mapa da distribuição das áreas com cana-de-açúcar e do município em relação æ̀s áreas com potenciais de produtividade de cana-de-açúcar está no Anexo 2. 


\subsubsection{Declividade das áreas ocupadas com cana-de-açúcar em relação às usinas e ao município}

Nota-se na Figura 8 que tanto as áreas somente com cana-de-açúcar e as áreas do município tem $50 \%$ de suas áreas com menos de aproximadamente $6 \%$ de declividade. O município possui áreas em classes que variam de 10 a 14\% de declividade ocorrendo com 7 a 10\% de freqüência em relação ao total de sua área.

Existe também um pico de ocorrência de cana-de-açúcar em áreas com declividade em torno de 12 a 13\%. Essas áreas provavelmente são ocupadas pelos fornecedores de cana-de-açúcar, que como visto anteriormente podem estar ocupando também as áreas distantes às usinas. A declividade tem relação direta com a tendência de mecanização da colheita da cana-de-açúcar, sendo portanto um dos fatores que determinarão um novo cenário de ocupação das terras do município por essa cultura.

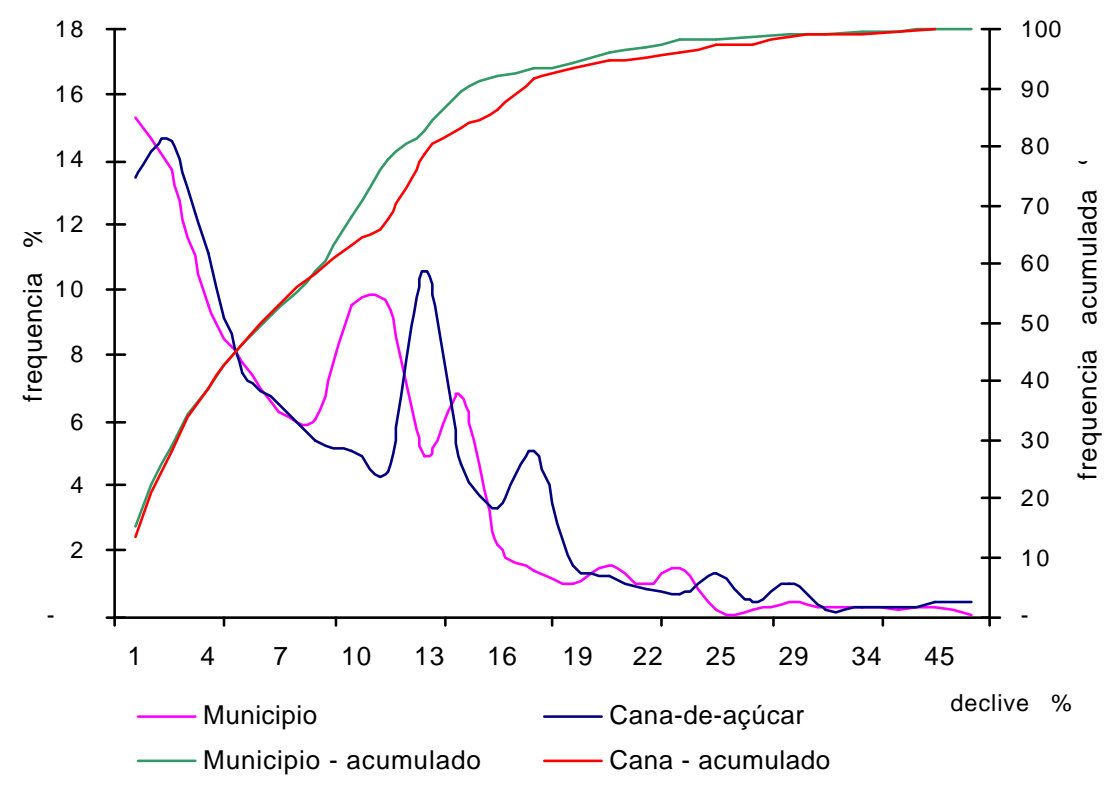

Figura 8 - Histograma de distribuição de freqüência das áreas com cana-deaçúcar em relação à declividade das áreas do município de Piracicaba. 
Segundo Sparovek et al. (1997a) e Sparovek et al. (1997b), a viabilidade da implantação da colheita mecanizada é possível com até $12 \%$ de declividade, os autores também concluíram que $30 \%$ da área total ocupada com cana-de-açúcar é considerada apta para colheita mecânica.

O mapa da distribuição das áreas com cana-de-açúcar e do município em relação à declividades está no Anexo 3.

\subsection{Modelo FAO}

Para caracterizar as condições climáticas da região, foi calculado o balanço hídrico seqüencial decendial segundo Thornthwait \& Mather (1955) para os ciclos de outubro a setembro dos anos de 1998 a 1999 e de 1999 a 2000 em função do ciclo da cana de ano (Figura 9). Calculou-se também o armazenamento de água nestes períodos considerando uma CAD de $100 \mathrm{~mm}$.

As precipitações totais no ciclo de outubro a setembro dos anos de 1998 a 1999, 1999 a 2000 foram de $1591 \mathrm{~mm}, 1128 \mathrm{~mm}$ respectivamente. Pelos gráficos dos balanços hídricos nota-se que o período de 1998-1999 apresentou o maior excedente hídrico no ciclo considerado, indicando um bom potencial de rendimento para a cana-de-açúcar neste ano. 
1999
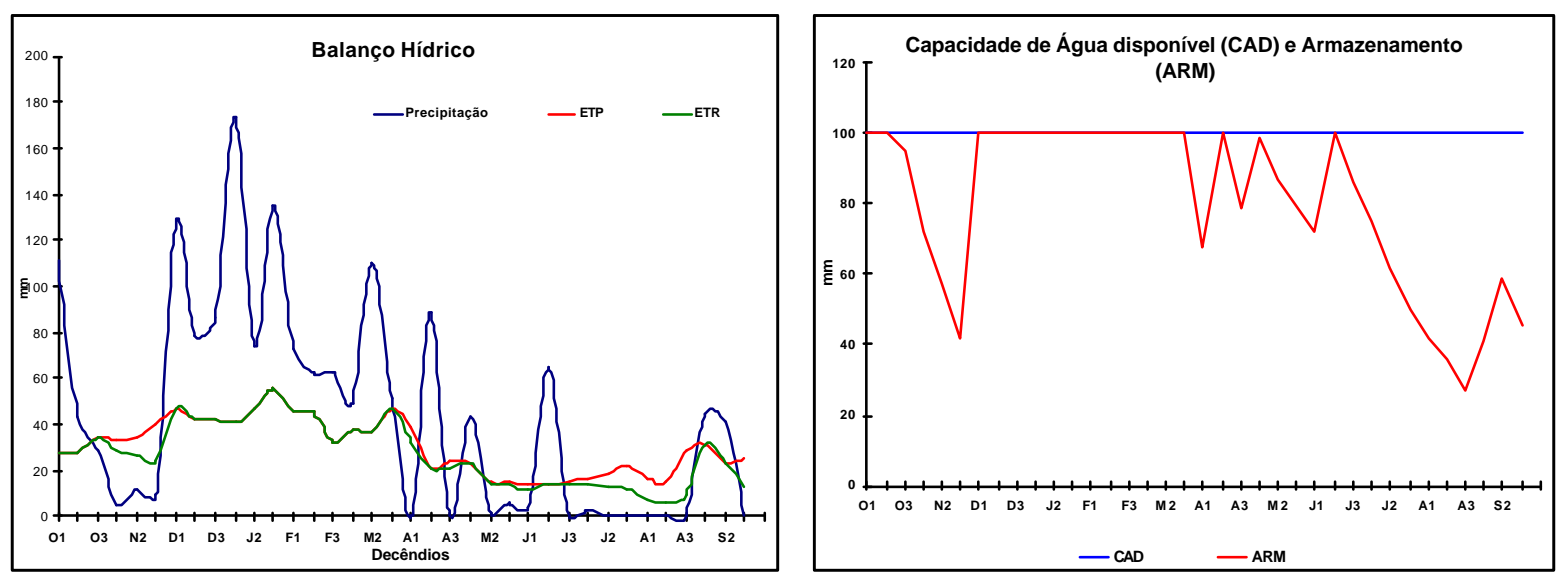

2000
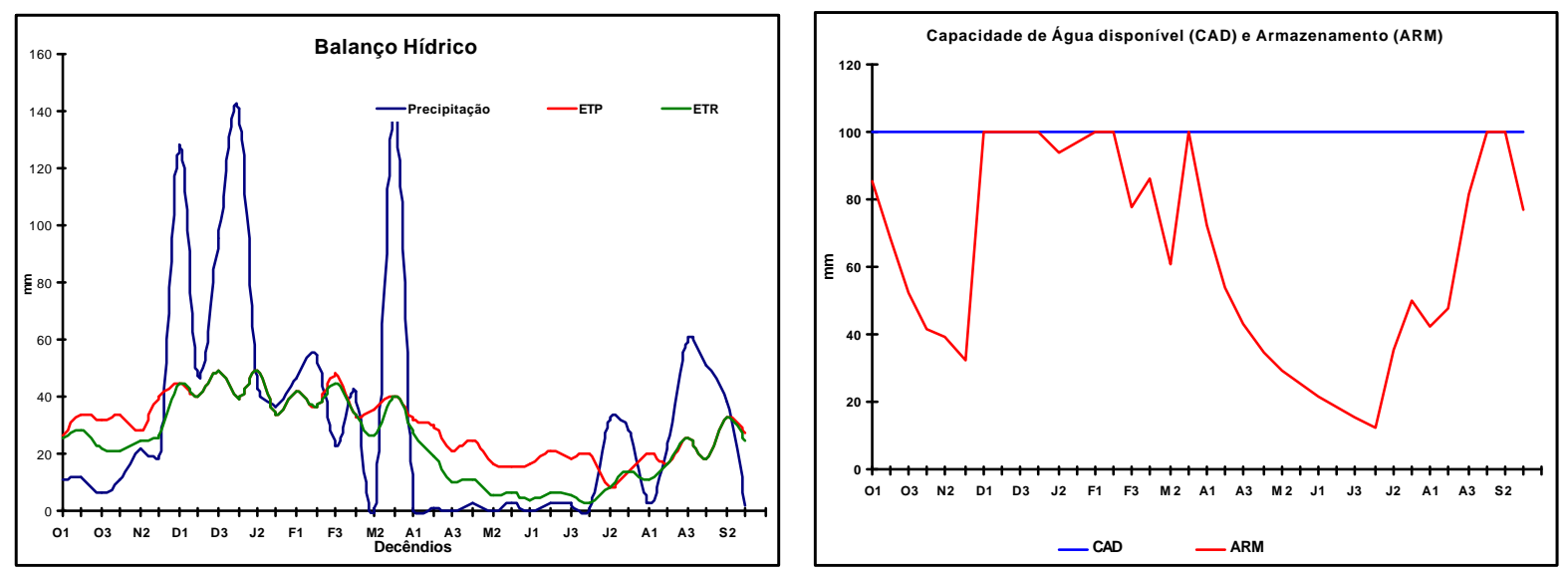

Figura 9 - Balanços hídricos seqüencial decendial e armazenamento de água no solo, segundo Thornthwait \& Mather (1955) para os ciclos de outubro a setembro de 1998 a 1999 e 1999 a 2000, para a região de Piracicaba. 


\subsubsection{Produtividade realizável}

Utilizou-se o modelo FAO para estimativa da produtividade realizável da cana-de-açúcar simulando uma cultura plantada mês a mês iniciando em janeiro até dezembro nos anos de 1999, 2000 e 2001, que leva em conta o balanço hídrico seqüencial decendial para cada período de um ano considerado, apresenta do na Figura 9.

Os resultados obtidos desta simulação estão na Tabela 9. Considerase que estes resultados representam uma cana-de-açúcar de primeiro corte, em um solo ideal, livre de pragas e doenças onde as perdas ocorreram apenas em função da falta de água.

Verifica-se que existe uma grande amplitude de valores de produtividade realizável dentro do mesmo ano. No ano de 2000 a produtividade mínima chegou a 32,18t.ha-1 de cana-de-açúcar com uma quebra de 79,6\%. A amplitude de valores de produtividade realizável em 1999 é de 97,0t.ha ${ }^{-1}$, em 2000 de 75,8t.ha ${ }^{-1}$ e em 2001 é de $71,0 t$.ha ${ }^{-1}$. Esse é um comportamento que geralmente não se verifica no campo em uma cultura conduzida de forma adequada e homogênea. Essa grande variação mostra que o modelo tem alta sensibilidade para restrições hídricas, que é a condição climática preponderane considerada neste modelo. Mas a penalização pode estar sendo superestimada pelo modelo através de seus parâmetros.

O mesmo não acontece com a produtividade potencial, que basicamente é função da radiação e da temperatura. Este cálculo apresenta pouca variação durante o ano. Em 1999 a amplitude foi de 43,4t.ha ${ }^{-1}$, em 2000 foi de 35,7 t.ha ${ }^{-1}$ e em 2001 foi de apenas 23,0t.ha ${ }^{-1}$.

Tabela 9. Resultados obtidos para produtividade real estimada para época de corte/plantio mês a mês iniciando em janeiro até dezembro dos anos de 1999, 2000 e 2001. 


\begin{tabular}{|c|c|c|c|c|c|c|c|c|c|}
\hline & \multicolumn{3}{|c|}{$\begin{array}{c}1999 \\
\text {----- Produtividade ------ }\end{array}$} & \multicolumn{3}{|c|}{$\begin{array}{c}2000 \\
\end{array}$} & \multicolumn{3}{|c|}{$\begin{array}{c}2001 \\
\text {----- Produtividade }\end{array}$} \\
\hline & $\begin{array}{c}\text { Potencial } \\
\text { t.ha }^{-1}\end{array}$ & $\begin{array}{c}\text { Quebra } \\
\%\end{array}$ & $\begin{array}{l}\text { Realizável } \\
\text { t.ha }^{-1}\end{array}$ & $\begin{array}{c}\text { Potencial } \\
\text { t.ha }^{-1}\end{array}$ & $\begin{array}{c}\text { Quebra } \\
\%\end{array}$ & $\begin{array}{l}\text { Realizável } \\
\text { t.ha }^{-1}\end{array}$ & $\begin{array}{c}\text { Potencial } \\
\text { t.ha }^{-1}\end{array}$ & $\begin{array}{c}\text { Quebra } \\
\%\end{array}$ & $\begin{array}{c}\text { Realizável } \\
\text { t.ha }^{-1}\end{array}$ \\
\hline Jan & 151,90 & 15,6 & 128,2 & 120,94 & 49,2 & 61,44 & 151,07 & 30,6 & 104,89 \\
\hline Fev & 155,73 & 22,5 & 120,68 & 121,7 & 53,8 & 56,27 & 151,56 & 19,7 & 121,74 \\
\hline Mar & 156,79 & 8,1 & 144,03 & 119,26 & 20,1 & 95,27 & 154,75 & 19,1 & 125,12 \\
\hline Abr & 160,92 & 7,3 & 149,22 & 123,33 & 20,5 & 98,07 & 158,91 & 23,2 & 122,02 \\
\hline Mai & 165,73 & 6,6 & 154,72 & 122,07 & 23,4 & 93,49 & 162,44 & 15,6 & 137,03 \\
\hline Jun & 165,92 & 10,4 & 148,65 & 120,27 & 27,0 & 87,82 & 162,91 & 17,1 & 135,13 \\
\hline Jul & 145,36 & 23,2 & 111,60 & 120,02 & 51,1 & 58,66 & 160,92 & 27,9 & 116,03 \\
\hline Ago & 122,5 & 12,4 & 107,28 & 155,48 & 73,6 & 41,02 & 139,14 & 45,4 & 76,01 \\
\hline Set & 122,85 & 30,5 & 85,32 & 156,77 & 79,5 & 32,18 & 144,23 & 48,5 & 74,25 \\
\hline Out & 126,85 & 43,0 & 72,34 & 153,44 & 77,6 & 35,43 & 145,79 & 54,7 & 66,01 \\
\hline Nov & 126,86 & 58,0 & 52,94 & 154,39 & 37,1 & 97,17 & 146,79 & 54,2 & 69,84 \\
\hline Dez & 124,24 & 30,6 & 86,22 & 152,16 & 28,7 & 108,54 & 149,97 & 46,4 & 80,36 \\
\hline MPS $^{*}$ & 141,802 & 23,01 & 111,232 & 137,719 & 43,86 & 74,765 & 152,585 & 35,21 & 100,18 \\
\hline
\end{tabular}

* MPS = Média do Período de Safra, aqui considerado como sendo de março a dezembro

A média da safra é somente um indicativo por ser uma estimativa da produtividade da safra, uma vez que para ser mais preciso deveria ser feita a ponderação com as frações das áreas colhidas a cada mês.

Realizou-se uma comparação entre a produtividade média medida no campo obtida em diferentes meses com a produtividade obtida pelo modelo FAO. Adicionalmente, os resultados do modelo FAO foram ponderados pela pontuação do solo (Ps) do modelo de estimativa de produtividade relativa baseado em parâmetros do solo e pelo número de cortes, e que foi designada neste trabalho como FAO corrigida. Assim, a produtividade realizável, calculada pelo modelo da FAO, foi multiplicada pela pontuação do solo e por um fator de correção para o número de cortes da cana-de-açúcar, considerado como uma taxa de decaimento de $5 \%$ a cada corte (Nunes Júnior, 1987). 
A Figura 10 mostra que não há relação entre a produtividade média media nos diferentes meses e as produtividades da FAO e FAO corrigida para corte e parâmetros do solo.
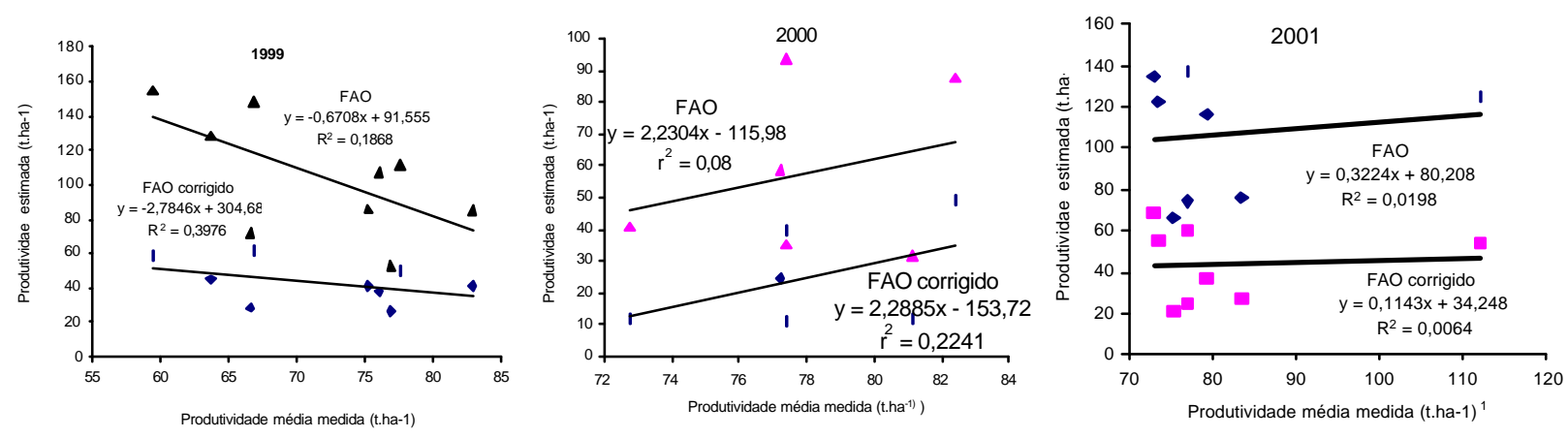

Figura 10-Comparação entre a produtividade média medida obtida em diferentes meses e a produtividade obtida pelo método FAO e também com a produtividade obtida pela FAO corrigida.

Isso demonstra que esse modelo não é adequado para estimativas regionais ou escalas menores para cana-de-açúcar. Isto se explica pelo fato do modelo FAO Ter sido concebido para gerar informações a nível continental com diferenças climáticas regionais contrastantes. A sua adequada utilização num escala local, para cana-de-açúcar, implica em ajustes de parâmetros e definições de intervalos de cálculos com dados experimentais.

O modelo $\mathrm{FAO}$ foi adequado para estimar a produtividade do girassol (Rolim, 2000), mas esta cultura é mais sensível à deficiência hídrica em seu curto ciclo, em comparação à cana -de-açúcar. Por outro lado a cana-de-açúcar é bastante tolerante a essas condições climáticas e apresenta grande capacidade de recuperação de seu crescimento nos períodos favoráveis.

Além disso, o modelo FAO deprecia a produtividade de forma irreversível no ciclo da cultura, o que não condiz com o comportamento da 
planta, que tem capacidade de realizar fotossíntese e armazenar reservas energéticas para ser utilizada no crescimento de colmos nos momentos mais propícios. 


\section{CONCLUSÕES}

- O modelo de estimativa de produtividade relativa com base em parâmetros do solo estima adequadamente a produtividade relativa da cana-de-açúcar.

- O modelo ganha precisão quando se trabalha em escalas maiores.

- O modelo de estimativa de produtividade relativa com base em parâmetros do solo é uma ferramenta de avaliação de terras que considera os diversos fatores envolvidos de forma holística e integrada de maneira mais apropriada que a analise estatística quando utilizada isoladamente.

- O modelo mostrou-se uma ferramenta útil para identificação do potencial produtivo de áreas para a cultura de cana-de-açúcar através da estimativa relativa de produtividade com base em mapa de solos.

- O modelo de estimativa de produtividade relativa com base em parâmetros do solo em atividades de diagnóstico e planejamento em escala regional. 


\section{REFERÊNCIAS BIBLIOGRÁFICAS}

ALFONSI, R.R.; PEDRO JUNIOR, M.J.; BRUNINI, O.; BARBIERI, V. Condições climaticas para cana-de-açúcar In: PARANHOS, S.B. (Coord.). Cana-de-açúcar: Cultivo e Utilização. Campínas: Fundação Cargill, 1987. v. I, p.42-55.

BARBIERI, V. Condicionamento climático da produtividade potencial da canade-açúcar (Saccharum spp): um modelo matemático-fisiológico de estimativa. Piracicaba, 1993. 142p. Tese (Doutorado) -Escola Superior de Agricultura "Luiz de Queiroz", Universidade de São Paulo.

BARBIERI, V.; VILLA NOVA, N.A. Climatologia e a cana-de-açúcar. In: PLANALSUCAR - Coordenadoria Regional Sul - COSUL, Araras, 1977. p.1-22.

BARBIERI, V.; TUON, R.L. Metodologia para estimativa da produtividade potencial de algumas culturas. ESALQ, Departamento de Física e Meteorologia, 1992.17p.

BASALDI, O.V.; FARIA, C.A.C.; NOVAES FILHO, R. Considerações sobre a dinâmica recente do complexo sucroalcooleiro no Estado de São Paulo, Informações Econômicas, v.26, n.4, p.21-29, 1996.

BEAUCLAIR, E. G. F. de Produtividade da cana-de-açúcar em função de alguns macronutrientes presentes no caldo e no solo. Piracicaba, 1994. 97p. Tese (Doutorado) Escola Superior de Agricultura "Luiz de Queiroz", Universidade de São Paulo.

BEAUCLAIR, E. G. F. de Relações entre algumas propriedades químicas do solo e a produtividade da cana-de-açúcar (Saccharum spp), através de regressão linear múltipla. Piracicaba, 1991. 96p. Dissertação (Mestrado) Escola Superior de Agricultura "Luiz de Queiroz", Universidade de São Paulo.

BERNARDES, M. S. Fotossíntese no dossel das plantas cultivadas. In: CASTRO, P.R.C. Ecofisiologia da produção agrícola. Piracicaba: POTAFOS, 1987. p.13-48. 
BERNARDES, M.S., SPAROVEK, G., ROSSETTO, R., FARAHT, M., VICCINO, J.R., STAPE, J.L., FILHO, V.A.C., HAMMERSCHLAG, N. Sistemas de produção para áreas com restrições à colheita mecanizada de cana -deaçúcar. In: SEMANA DE CANA-DE-AÇÚCAR EM PIRACICABA, 3, Piracicaba, 1998. Anais. Piracicaba: STAB, 1998. p.35-37.

BERNARDES, M. S. SPAROVEK, G.; BARBOSA, M.; SADER, S.L. Comparação entre a produtividade real e a simulada através de modelo matemático avaliada em cinco variedades de cana-de-açúcar (Saccharum spp) In: CONGRESSO CONGRESSO NACIONAL DA STAB, 8., Recife, 2002. Anais. Piracicaba: STAB, 2002. p477-482.

BITTENCOURT, V.C. de; JOÃO, J.; CLEMENTE, J.L.; CARMELLO, Q.A. de C.; BEAUCLAIR, E.F. de Produtividade da cana-de-açúcar e suas relações com a fertilidade dos solos e manejo da cultura. STAB: Açúcar, Álcool e Subprodutos, v.9, n.2, p.10-14, mai/ago 1990.

BITTENCOURT, V.C.; CLARET, A.C.; CARMELLO, Q. A. de C.; CARVALHO, A J. L. Produtividade da cana-de-açúcar e fertilidade do solo. STAB: Açúcar, Álcool e Subprodutos, v.8, n.20, p.41-45. nov/dez 1989.

BOS, J. VAN DEN; RABBINGE, $R$ Simulation of the fluctuations of the grey larch bud moth. Wageningen, The Netherlands: Centre for Agricultural Publishing and Documentation, 1976. 83p.

BOUMA, J. The role of quantitative approaches in soil science when interacting with stakeholders. Geoderma, n.78, p.1-12, 1997.

BRADY, N.C Natureza e propriedade dos solos. Trad. Antônio B. N. Figuyeiredo Filho, 6. ed., Rio de Janeiro. Freitas Bastos, 1983. 647p.

CÂMARA, G. Anatomia de Sistemas de Informações Geográficas: Visão atual e perspectivas de evolução. In: ASSAD, E.D. ; SANO, E.E., (Coord.). Sistemas de Informações Geográficas: aplicações na agricultura. Planaltina: Embrapa-CPAC, 1993. 274p.

CHINENE, V.R.N. The Zambian land evaluation system (ZLES). Soil Use and Management v.7, p.21-30, 1991.

DANIELS, J.; ROACH, B.T. Taxonomy and evolution. In: HEINZ, D.J. (Ed.) Sugarcane improvement through breeding. Amsterdam: Elsevier, 1987. p.7-84. 
DIAS, F.L.F.; MAZZA, J.A.; MATSUOKA, S.; PERECIN, D.; MAULE, R.F. Produtividade da cana-de-açúcar em relação ao clima e solos da região noroeste do Estado de São Paulo. Revista Brasileira de Ciência do Solo, v.23, n.3, p.627-634, 1999.

DIEPEN VAN, C.A.; VANKEULEN, H.; WOLF, J. AND BERKHOUT, J. A.A. Land evaluation: from intuition to quantification. In: STEWART, B.A. (Ed.). Advances in Soil Science. New York: Springer, 1991. p.139-204.

DOOREMBOS, J.; KASSAN, A.H. Yield response to water. Rome: FAO, 1979, 212p. (FAO. irrigation and drainage paper, 33).

DOOREMBOS, J.; PRUITT, W.D. Guidelines for predicting crop water requirements. Rome: FAO, 1977, 179p. (irrigation and drainage paper, 24).

EPSTEIN, E. Nutrição mineral das plantas: princípios e perspectivas. Trad. Malavolta E., Rio de Janeiro: Editora da Universidade de São Paulo, 1975, $344 p$.

FAO A framework for land evaluation. Rome, 1976. 153p. (FAO. Soils Bulletin 32).

FIGUEIREDO, P.; LANDELL, M.G.A.; CAMPANA, M.P. Cana-de-açúcar. (compact disc) 6. ed. Campinas: IAC, 1995. (IAC. Boletim 200)

FNP Consultoria \& Comércio., Agrianual 2000. Anuário de Agricultura Brasileira. São Paulo, 1999. p. 80-81.

FRANCE, J.; THORNLEY, J.H.M. Mathematical models in agriculture: A quantitative approach to problems in agricultural and related sciences. London: Butterworth , 1984. 249 p.

GLOVER, J. Practical theoretical assessments of sugarcane yield potential in Natal. Proceeding of the South African Sugar Technologists Association, v.46, p.138-141, 1972.

GONÇALVES, J.S.; SOUZA, S.A.M. Proibição da queima no Estado de São Paulo: simulações dos efeitos por área cultivada e na demanda pela força de trabalho. Informações econômicas, v.28, n.3, p.21-40, 1998.

HAAG, H.P.; DECHEN, A.R.; CARMELLO, Q.A.C. Nutrição mineral da canade-açúcar In: PARANHOS, S.B. (Coord.). Cana-de-açúcar: Cultivo e Utilização. Campínas: Fundação Cargill, v.2, 1987, p.88-162. 
HANKS, R. J. ; RITCHIE, J.T. Modelling plants and soil systems. Madison: American Society of Agronomy, Crop Science society of America, Inc./Soil Science Society of America, 1991. 387 p. (Agronomy, 31)

HUMBERT, H.P. The growing of sugarcane. Amsterdam. Elsevier, 1968. $779 p$.

HURNI, H. Assessing sustainable land management (SLM). Agriculture, Ecosystems and Environment v.81, p.83-92. 2000.

IBGE http://www.ibge.gov.br (30 dez 2002)

IEA, http://www.iea.sp.gov.br/fbcoiea.htm (30 dez 2002)

INMAN-BAMBER, N. G., A growth model for sugarcane based on a simple carbon balance and the ceres-maize water balance. South African Journal Plant and Soil, v.8, p93-99, 1991.

INMAN-BAMBER, N. G.; THOMPSON, G. D. Models of dry matter accumulation by sugarcane. Proceeding of the South African Sugar Technologists Association, v.63, p.212-216, 1989.

JOAQUIM, A.C.; BELLINARO, I.F.; DONZELLI, J.L.; QUADROS, .A.C.; BARATA, M.Q.F. Potencial e manejo de solos cultivados com cana-deaçúcar In: SEMINÁRIO COPERSUCAR DE TECNOLOGIA AGRONÔMICA, 6., Piracicaba, 1994. Anais. Piracicaba: Copersucar. 1994. P.1-9.

JONES, C.A.; KINIRY, J. R. CERES-MAIZE model: a simulation model of maize growth and development. Texas: A\&M University Press, 1986. 194p.

KOFLER, N.F.; DONZELI, P.L. avaliação dos solos brasileiros para a cultura da cana-de-açúcar In: PARANHOS, S.B. (Coord.). Cana-de-açúcar: Cultivo e Utilização. Campínas: Fundação Cargill, 1987, v.2. p.19-41.

LAFFELAAR, P.A. (Ed.) On systems analysis and simulation of ecological processes: with exemples in CSMP and FORTRAN (current issues in ecology; VI). The Netherlands: Kluwer Academic, 1993. 293p.

LEITE, R.C.C. Pró-alcool: a única alternativa para o futuro. Campinas: UNICAMP, 1987. 86p.

LEPSCH, I. Influência dos fatores edáficos na produção. In: CASTRO, P.R.C.; FERREIRA,,S.O.; YAMADA, T.; (Coord.). Ecofisiologia da produção. Piracicaba: Associação Brasileira para Pesquisa da Potassa e do Fosfato (POTAFOS), 1897. p.83-98. 
LEVY, M.C.T.C. Avaliação de cenários da produção agrícola visando a sustentabilidade do uso das terras de Piracicaba (SP). Piracicaba, 1995. 104p. Dissertação (Mestrado) Escola Superior de Agricultura "Luiz de Queiroz", Universidade de São Paulo.

LEVY, M.C.T.C.; SPAROVEK, G. Avaliação do potencial de utilização das terras de Piracicaba para o cultivo de cana-de-açúcar pela estimativa do seu risco de degradação. In: CONGRESSO BRASILEIRO DE CIENCIA DO SOLO, 25., Viçosa, 1995. Ed. Departamento de Solos, Anais, Viçosa: 1995. v.4, p.175-177.

LOPES, A.S. Manual internacional de fertilidade do solo. 2. ed. Piracicaba: POTAFOS, 1998. 177p.

MAGALHÃES, A.C.N. Ecofisiologia da cana-de-açúcar: aspectos do metabolismo do carbono na planta. . In: CASTRO, P.R.C.; FERREIRA,,S.O.; YAMADA, T.; (Coord.). Ecofisiologia da produção. Piracicaba: Associação Brasileira para Pesquisa da Potassa e do Fosfato (POTAFOS), 1897. p.113-118.

MALAVOLTA, E. Manual de química agrícola, nutrição de plantas e fertilidade do solo. São Paulo: Ed. Agronômica Ceres, 528p. 1976.

MALAVOLTA, E. Nutrição mineral e adubação da cana-de-açúcar., Piracicaba: Ed. Ultrafértil Departamento de serviços técnicos agronômicos, 1982. 80p.

MALAVOLTA, E.; HAAG, H.P.; MELLO, F.A.F.; BRASIL SOBRINHO, M.O.C. Nutrição mineral e adubação de plantas cultivadas. São Paulo: Ed. Pioneira, 1974. 752p.

MATIOLI, C.S. Irrigação suplementar de cana-de-açúcar: modelo de análise de decisão para o Estado de São Paulo. Piracicaba, 1998. 122p. Tese (Doutorado) Escola Superior de Agricultura "Luiz de Queiroz", Universidade de São Paulo.

MATSUOKA, S.; LAVORENTI, N.A.; MENEZES, L.L.; SALIBE, A.C.; GHELLER, A.C.A.; ARIZONO, H. Novas variedades de cana-de-açúcar para a região Oeste do Estado de São Paulo. In: CONGRESSO NACIONAL DA STAB, 7., Londrina, 1999. Anais. Piracicaba: STAB, 1999. p.34-39.

MAULE, R.F.; MAZZA, J.A.; MARTHA JUNIOR, G.B. Produtividade agrícola de cultivares de cana-de-açúcar em diferentes solos e épocas de colheita. Scientia Agricola, v.58, n.2, p.295-301, abr/jun. 2001 
McCREE, K. J. An equation for the rate of respiration of white clover plants growth under controlled conditions. In: SETLIK, I., (Ed.). Prediction and measurement of photosynthetic productivity. Wageningen, Pudoc: Elsevier, 1970. p.221-229.

MURAMOTO, J.; SPAROVEK, G.; LEPSCH, I.; VIDAL TORRADO, P. Adequação do uso das terras de Piracicaba (SP) In: CONGRESSO BRASILEIRO DE CIÊNCIA DO SOLO, 24., Resumos Goiânia: SOCIEDADE BRASILEIRA DE CIÊNCIA DO SOLO. v.2, 1993, p405-406

NUNES JUNIOR, D. Variedades da cana-de-açúcar In: PARANHOS, S.B. (Coord.). Cana-de-açúcar. Cultivo e Utilização. Campinas, Fundação Cargill, v.2., 1987, p.187-259.

OLIVEIRA, J.B., \& PRADO, H. Carta pedológica Semi-detalhada do Estado de São Paulo. Folha de Piracicaba, Campinas, Instituto Agronômico e Instituto Geográfico e Cartográfico. 1989 (escala 1:100.000).

OMETTO, J.C. Parâmetros meteorológicos e a cultura da cana-de-açúcar. Piracicaba, 1980. 17p. Escola Superior de Agricultura "Luiz de Queiroz", Universidade de São Paulo.

OMETTO, J.C. Registros e estimativas de parâmetros meteorológicos da região de Piracicaba, SP. Piracicaba: Fealq. 76p. 1989.

OMETTO, J.C. Uma equação para a estimativa de evapotranspiração potencial; sua aplicação no cálculo das necessidades hídricas e do rendimento agro-indstrial da cana-de-açúcar na região de Piracicaba (SP). Piracicaba, 1974. 129p. Tese (Livre Docência) Escola Superior de Agricultura "Luiz de Queiroz", Universidade de São Paulo.

ORGANIZAÇÃO DOS PLANTADORES DE CANA-DE-AÇÚCAR DO ESTADO DE SÃO PAULO. Queima da cana-de-açúcar, informações disponíveis. Câmara paulista do setor sucroalcoleiro, Piracicaba: Orplana, 1996. 18p. (Boletim)

ORLANDO FILHO, J. Nutrição e adubação de cana-de-açúcar no Brasil. Piracicaba: Instituto do Açúcar e do Álcool, 1983. 369p.

ORLANDO FILHO, J.; MACEDO, N.; TOKESHI, H. Seja doutor do seu canavial. Informações Agronômicas, n.67, p.17, set. 1994. (Arquivo do Agrônomo, $6)$. 
ORLANDO FILHO, J.; SILVA, L.C.F.; LAVORENTI, N.A.; Adubação PK em cana-de-açúcar cultivada em dois espaçamentos de plantio. STAB, Açúcar Álcool e Subprodutos, v.9, n.2, p.15-21, 1990.

PARANHOS, S.B. (Coord.). Cana-de-açúcar. Cultivo e Utilização. Campinas, Fundação Cargill, v.2, 1987. 856p.

PENATTI, C.P. Uso de ácido fosfórico ou superfosfato triplo como fonte de fósforo para a cultura da cana-de-açúcar. Piracicaba, 1991. 105p. Dissertação (Mestrado) - Escola Superior de Agricultura "Luiz de Queiroz", Universidade de São Paulo.

PENNING de VRIES, F.W.T. System analysis and models of crop growth. In: PENNING DE VRIES, F.W.T.; VAN LAAR, H.H. (Ed.). Simulation of plant growth and crop production. Pudoc, Wageningen: Elsevier, 1982. p.9-22. (Simulation monographs).

PEREIRA, A.R.; MACHADO, E.C. Um simulador dinâmico do crescimento de uma cultura de cana-de-açúcar. Bragantia, v.45, n.1, p.107-122, 1986.

PEREIRA, A. R. Balanço de Carbono: Uso de Modelos e Simulação. In: CROCOMO, O.J.; SILVEIRA, J.A.G. da (Ed.) SEMINÁRIO DE BIOTECNOLOGIA AGRíCOLA, 5., Piracicaba, 1987. Anais. Piracicaba: CEBTEC/FEALQ, 1987. p.189-207.

PEREIRA, A.R.; MACHADO, E.C. Análise quantitativa do crescimento de comunidades vegetais. Campinas: Instituto Agronômico, 1987. 33p.

PEREIRA, A. R.; VILLA NOVA, N. A.; SEDIYAMA,G.C. Evapo(tranpi)ração. Piracicaba: FEALQ, 1997. 183p.

PEREIRA, A. R.; ANGELOCCI, L.R.; SENTELHAS, P.C. Agrometeorolgia: fundamentos e aplicações práticas, Guaiba: Ed. Agropecuária, 2002. 478p.

RAIJ, B VAN. Avaliação da fertilidade do solo. 2.ed. Piracicaba: Instituto da Potassa e Fosfato, 1981. 142p.

RAIJ, B. VAN.; CANTARELLA, H.; QUAGGIO, J.A.; FURLANI, A.M.C. (Ed.). Recomendações para adubaçãoe calagem para o Estado de São Paulo. 2.ed. Campinas: Instituto Agronômico de Campinas, 1985. 285p. (Boletim Técnico, 100). 
RAIJ, B. VAN; QUAGGIO, J.A. ; CANTARELLA, H.; FERREIRA, M.E.; LOPES, A.S.; BATAGLIA, O.A. Análise química do solos para fins de fertilidade. Campinas: Fundação Cargil, 165p., 1987.

RANIERI, S.B.L.; SPAROVEK, G. Evolução do uso das terras do município de Piracicaba (1962-1991) In: CONGRESSO BRASILEIRO DE CIÊNCIA DO SOLO, 24., Goiânia, 1993, Resumos, Goiânia: SOCIEDADE BRASILEIRA DE CIÊNCIA DO SOLO. v.2, 1993, p.387-388

ROJAS, J.S.D.; BARBIERI, V. modelo agrometeorológico de estimativa da produtividade da cana-de-açúcar. Revista de Agrometeorologia, v.7, n.1, p.67-73, 1999.

ROLIN, G. S.; SENTELHAS, P.C.; BARBIERI, V. Planilhas no ambiente excel para os cálculos de balanços hídricos: normal, seqüencial, de cultura e de produtividade real e potencial. Revista de Agrometeorologia, v.6, n.1, p.133-137, 1998.

ROLIN, G. S.; Ajuste e comparação de modelos de simulação de produtividade para a determinação do risco climático da cultura do girassol (Helianthus annus L) Piracicaba, 2000. 109p. Dissertação (Mestrado) Escola Superior de Agricultura "Luiz de Queiroz", Universidade de São Paulo.

ROSENFELD, U. Determinação do período crítico de deficiência hídrica para a cultura da cana-de-açúcar (Saccharum spp), ciclo da cana planta. Piracicaba, 1989. 89p. Dissertação (Mestrado) Escola Superior de Agricultura "Luiz de Queiroz", Universidade de São Paulo.

ROSSITER, D.G. A theoretical framework for land evaluation. Geoderma, v.72, p.165-190, 1996.

SAS INSTITUTE. SAS Language and procedures: Usage, version 6.11. Cary, NC: Sas Institute, 1989. 638p.

SCARDUA R.; ROSENFELD, U. Irrigação da cana-de-açúcar In: PARANHOS, S.B. (Coord.). Cana-de-açúcar: Cultivo e Utilização. Campínas: Fundação Cargill, 1987. v. I, p.373-431.

SCARPARI, M.S. Modelo para a previsão da produtividade da cana-de-açúcar (Saccharum spp) através de parâmetros climáticos. Piracicaba, 2002. 79p. Dissertação (Mestrado) - Escola Superior de Agricultura "Luiz de Queiroz", Universidade de São Paulo.

SINGELS, A.K.; BEZUIDENHOUT, C.N. IRRICANE: A simple computerised irrigation scheduling method for sugarcane. Proceeding of the South African Sugar Technologists Association, v.72, p.117-122, 1989. 
SINGELS, A.K.; DONALDSON, R.A. A simple model for unstressed canopy development. Proceeding of the South African sugar technology association, v.74: p.151-154, 1998.

SPAROVEK, G.; LEPSCH, I.F. Diagnóstico de uso e aptidão das terras agrícolas de Piracicaba. In: TAUK-TORNISIETO, S.M.; GOBBI, N.; FORESTI,C.; LIMA, S.T. de. Análise ambiental: estratégias e ações. São Paulo: Queiroz, 1995. p.273-279.

SPAROVEK, G.; PEREIRA, J.C.; ALLEONI, L.R.F.; ROSSETTO, R. Aptidão das terras de Piracicaba (SP) para o corte mecanizado de cana-de-açúcar. STAB, v.15, n.5, p.6-9, 1997a.

SPAROVEK, G.; VAN LIER, Q.; ALOISI, R.R.; VIDAL TORRADO, P. Previsão do rendimento de uma cultura em solos de Piracicaba em função da erosão. Revista Brasileira de Ciência do Solo, v.17, p.465-470, 1993.

SPAROVEK, G; ALLEONI, L.R.F.; ROSSETTO, R. Avaliação das terras de Piracicaba (SP) demonstra dificuldade de adaptação da cana-de-açúcar às mudanças da legislação ambiental. Boletim da Sociedade Brasileira de Ciência do Solo, v.22, n.2, p.72-76, 1997b.

SPAROVEK, G.; COOPER, M.; DOURADO NETO, D; MAULE, R. F.; VIDAL TORRADO, PABLO; PIMENTA, L. F. M.; MARTINS, S. P.; TERAMOTO, E. R.; SILVA, A. C.; VAN DE STEEG, J.; SCHUNG, E. The land resource information and suitability system for family agriculture (LARISSA), developed for the brazilian agrarian reform. In: Journal of Agriculture in the Tropics and Subtropics, Witzenhausur, v.103, n.1, p. 47-59, 2002.

THOMPSON,G.D. water use by sugarcane. The South African Sugar Journal, v.60, p.593-600; p.627-635, 1976.

THORNLEY, J. H. M. Respiration, gowth and maintenace in plants. Nature, v.227, p.304-305, 1970.

THORNLEY, J.H.M. Mathematical models in plant physiology: a quantitative approach to problems in plant and crop physiology, London: Academic Press., 1976. 318p.

THORNTHWAIT, C. W.; MATHER, R.J. The water balance. New Jersey: Laboratory of Climatology, v.8, 1955, 104p (Publication in Climatology).

TISDALE S.; NELSON W., soil fertility and fertilizers. 3. ed., New York: Collier MacMillan International editions, 1975, 694p. 
WIT, C.T. de Photosynthesis of leaf canopies. Agric. Res. Rep., v.663, p.1-57, 1965.

WIT, C.T. de; GOUDRIAAN, J. Simulation of ecological processes. Wageningen, The Netherlands: Centre for Agricultural Publishing and Documentation, 1974. 159p.

WIT, C.T. de Simulation of assimilation, respiration and transpiration of crops. Wageningen, The Netherlands: Centre for Agricultural Publishing and Documentation, 1978. 141p. 


\section{APÊNDICES}




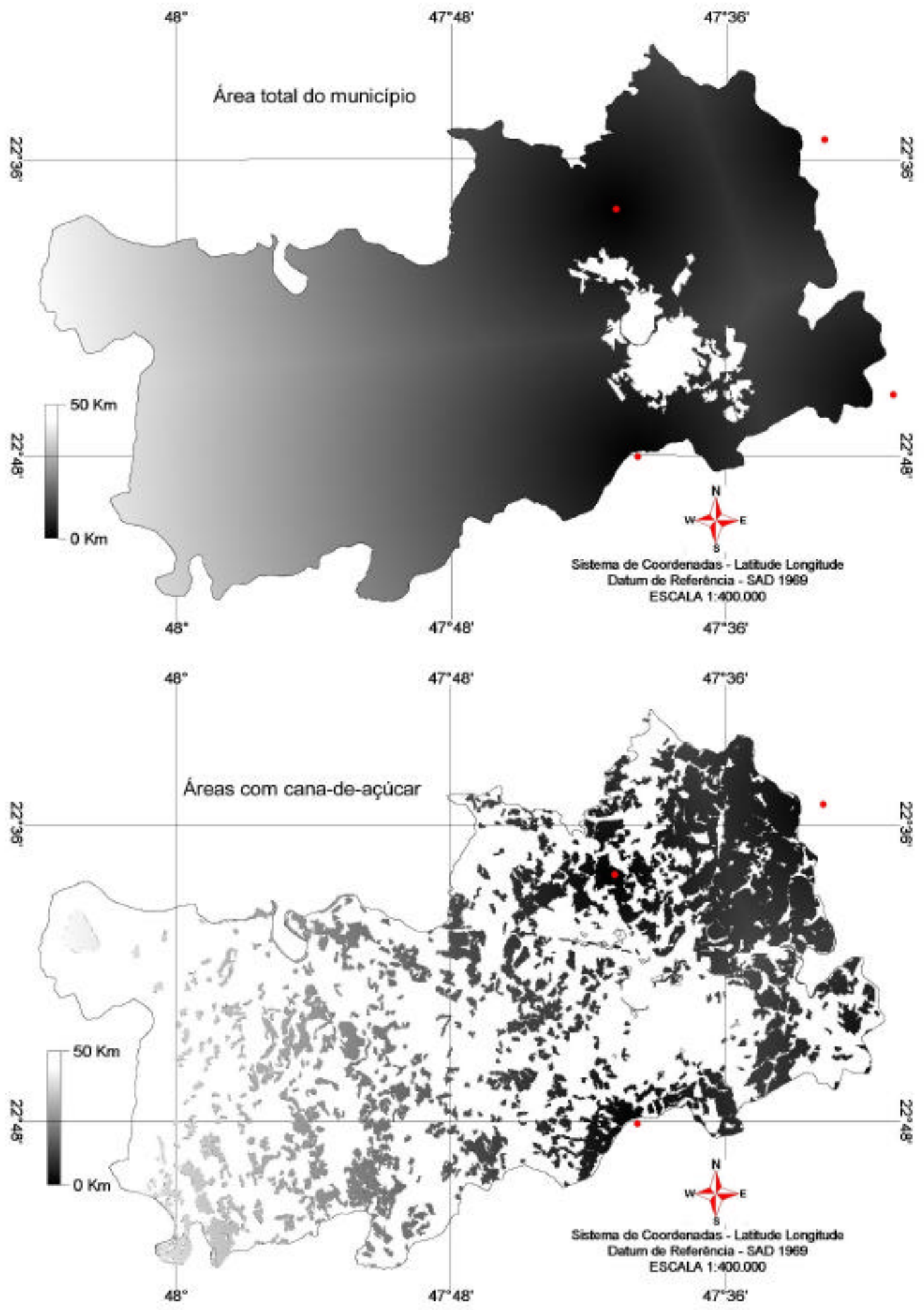



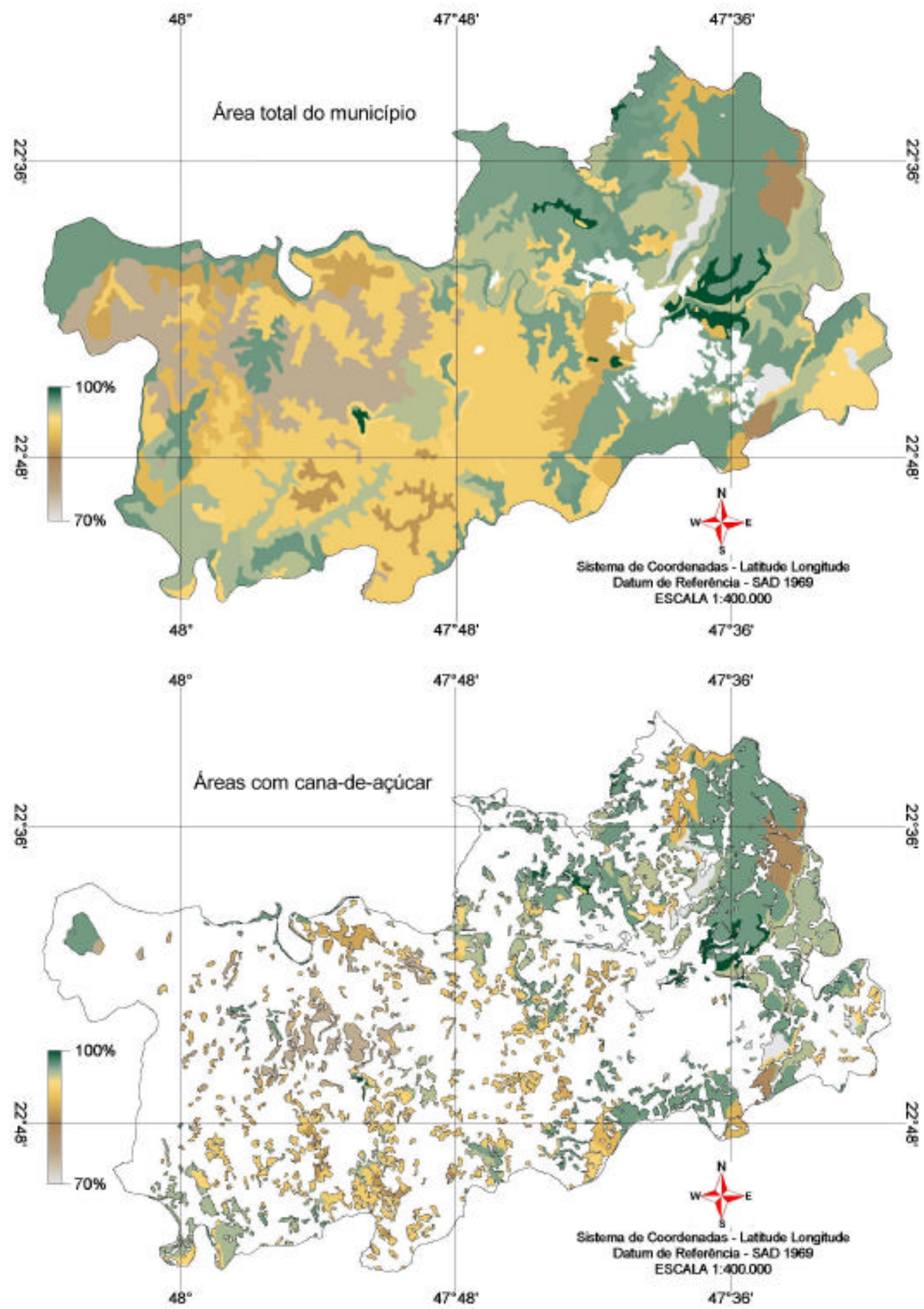


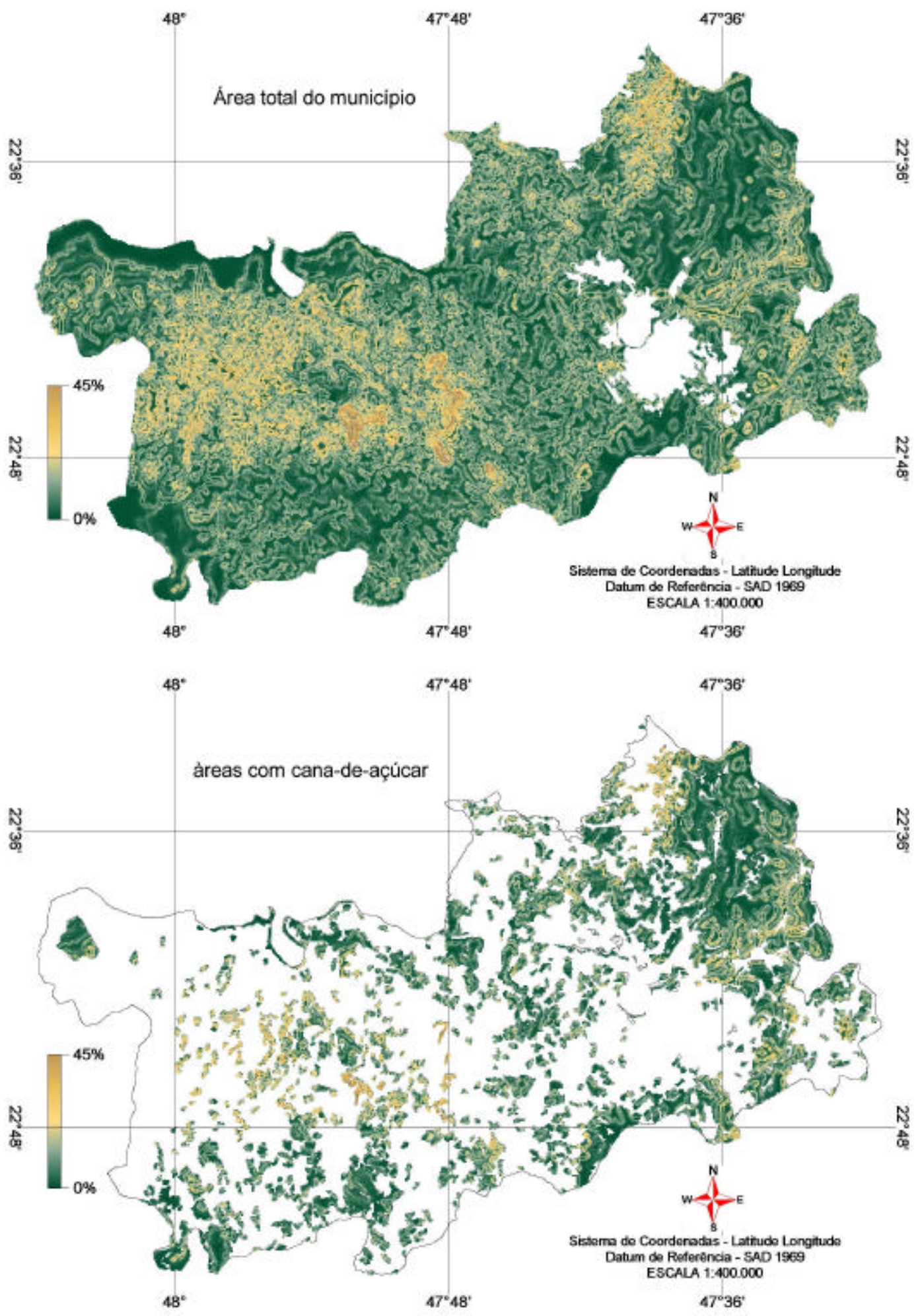




\begin{tabular}{|c|c|c|c|c|c|c|c|c|c|c|c|c|c|c|c|c|c|c|c|c|c|}
\hline nome & ano 1999 & & & & & & & teores & & & & & pontuação & & & & & & & & \\
\hline Fazenda & Solo & zona & talhão & horiz & coleta & Variedade & Estágio & $\mathrm{K}$ & $\mathrm{P}$ & T & $v_{A}$ & Arg & $\mathrm{K}$ & \begin{tabular}{|l|l|}
$P$ \\
\end{tabular} & 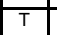 & $\mathrm{v}$ & Arg & NP & Ps & prod estima & prod medid \\
\hline ST.ROSA II & LE2+LE3 & 2 & 17 & A & 01/01/99 & SP79-1011 & $60 \mathrm{C}$ & 5,4 & \begin{tabular}{|l|}
14 \\
\end{tabular} & 32 & \begin{tabular}{l|l}
43 \\
43
\end{tabular} & 51 & 0,90 & \begin{tabular}{|l|}
0,47 \\
\end{tabular} & 0,16 & 1,05 & 1,00 & 10,68 & 21,46 & 86,37 & 108,42 \\
\hline ST.ROSA II & LE2+LE3 & 2 & 17 & B & $01 / 01 / 99$ & SP79-1011 & $60 \mathrm{C}$ & 4,4 & \begin{tabular}{|l|}
22 \\
\end{tabular} & 34 & \begin{tabular}{l|l}
4 \\
1
\end{tabular} & 63 & 0,73 & 0,68 & 0,17 & 1,02 & 1,00 & 10,79 & & & \\
\hline ST.ROSA II & LE2+LE3 & 5 & 31 & A & \begin{tabular}{|l|}
$01 / 01 / 99$ \\
\end{tabular} & RB72-454 & $20 . \mathrm{C}$ & 5,6 & 8 & 30 & \begin{tabular}{l|l}
26 &
\end{tabular} & 51 & 0,93 & 0,31 & 0,15 & 0,18 & 1,00 & 12,82 & 26,46 & 90,57 & 80,65 \\
\hline ST.ROSA II & LE2+LE3 & 5 & 31 & B & \begin{tabular}{|l|}
$01 / 01 / 99$ \\
\end{tabular} & RB72-454 & $20 . C$ & 3,8 & 4 & 37 & 45 & \begin{tabular}{l|l}
63 \\
\end{tabular} & 0,63 & \begin{tabular}{|l|l}
0,17 \\
\end{tabular} & 0,19 & 1,08 & 1,00 & 13,63 & & & \\
\hline ST.ROSA II & LE2+LE3 & 6 & 34 & A & \begin{tabular}{|l|}
$01 / 01 / 99$ \\
\end{tabular} & SP80-1842 & $20 . \mathrm{C}$ & 2,8 & \begin{tabular}{|l|}
16 \\
\end{tabular} & 75 & 30 & 51 & 0,47 & 0,53 & 0,50 & $\overline{0,21}$ & 1,00 & 13,06 & 27,98 & 91,85 & 81,5 \\
\hline ST.ROSA II & LE2+LE3 & 6 & 34 & B & 01/01/99 & SP80-1842 & $20 . \mathrm{C}$ & 1,3 & 6 & 62 & 45 & 63 & 0,22 & 0,25 & \begin{tabular}{|l|}
0,87 \\
\end{tabular} & 1,08 & 1,00 & $\mid 14,91$ & & & \\
\hline ST.ROSA II & LE2+LE3 & 6 & 41 & A & 01/01/99 & SP80-1842 & $20 . \mathrm{C}$ & 4,4 & \begin{tabular}{|l|}
16 \\
\end{tabular} & 29 & 24 & 51 & 0,73 & 0,53 & 0,15 & 0,17 & 1,00 & 12,60 & 22,58 & 87,30 & 68,66 \\
\hline ST.ROSA II & LE2+LE3 & 6 & 41 & B & |01/01/99 & $\begin{array}{l}\text { SP80-1842 } \\
\text { (1) }\end{array}$ & $20 . C$ & 1,2 & \begin{tabular}{|l|}
12 \\
\end{tabular} & 25 & 12 & 63 & 0,20 & 0,42 & 0,13 & 0,09 & 1,00 & \begin{tabular}{|l|}
9,98 \\
\end{tabular} & & & \\
\hline ST.ROSA II & LE2+LE3 & 7 & 60 & A & \begin{tabular}{|l|}
$10 / 09 / 99$ \\
\end{tabular} & SP79-1011 & $60 . C$ & 0,3 & \begin{tabular}{l|l}
6 \\
\end{tabular} & 49 & 63 & 51 & 0,05 & 0,25 & 0,25 & 0,76 & \begin{tabular}{|l|l|}
1,00 \\
\end{tabular} & \begin{tabular}{|l|}
7,07 \\
\end{tabular} & 13,31 & 79,51 & 63,80 \\
\hline ST.ROSA II & LE2+LE3 & 7 & 60 & $\mathrm{~B}$ & \begin{tabular}{|l|}
$10 / 09 / 99$ \\
\end{tabular} & SP79-1011 & $60 . C$ & 0,8 & \begin{tabular}{|l|}
13 \\
\end{tabular} & 35 & 11 & 63 & 0,13 & 0,44 & 0,18 & 0,08 & 1,00 & \begin{tabular}{|l|}
6,24 \\
\end{tabular} & & & \\
\hline ST.ROSA II & LE2+LE3 & 8 & 63 & A & \begin{tabular}{|l|}
$30 / 12 / 99$ \\
\end{tabular} & RB72-454 & $50 . \mathrm{C}$ & 6,2 & \begin{tabular}{|l|}
60 \\
\end{tabular} & 37 & 24 & 51 & 1,00 & 1,00 & 0,19 & 0,17 & 1,00 & 12,62 & 22,51 & 87,24 & 70,04 \\
\hline ST.ROSA II & LE2+LE3 & 8 & 63 & B & \begin{tabular}{|l|}
$30 / 12 / 99$ \\
\end{tabular} & RB72-454 & $50 . \mathrm{C}$ & 2,3 & 44 & 30 & 16 & \begin{tabular}{l|l}
63 \\
\end{tabular} & 0,38 & 1,00 & 0,15 & $\overline{0,11}$ & 1,00 & \begin{tabular}{|l|}
9,89 \\
\end{tabular} & & & \\
\hline ST.ROSA II & LE2+LE3 & 8 & 64 & A & 30/12/99 & RB72-454 & $50 \mathrm{C}$ & 2,5 & \begin{tabular}{|l|}
99 \\
\end{tabular} & 28 & 10 & 51 & 0,42 & 1,00 & 0,14 & 0,07 & 1,00 & \begin{tabular}{|l|}
9,68 \\
\end{tabular} & $\mid 17,46$ & 82,99 & 115,8 \\
\hline ST.ROSA II & LE2+LE3 & 8 & 64 & $B$ & 30/12/99 & RB72-454 & $50 \mathrm{C}$ & 1,0 & \begin{tabular}{|l|}
18 \\
\end{tabular} & 42 & 9 & \begin{tabular}{l|l|}
63 \\
\end{tabular} & 0,17 & 0,58 & 0,21 & 0,06 & 1,00 & \begin{tabular}{|l|}
7,77 \\
\end{tabular} & & & \\
\hline ST.ROSA II & LE2+LE3 & 9 & 76 & A & 01/01/99 & RB72-454 & $20 . \mathrm{C}$ & 3,0 & 4 & 21 & 28 & 51 & 0,50 & \begin{tabular}{|l|}
0,17 \\
\end{tabular} & 0,11 & 0,20 & 1,00 & 10,41 & \begin{tabular}{|l|}
19,75 \\
\end{tabular} & 84,92 & 94,0 \\
\hline ST.ROSA II & LE2+LE3 & 9 & 76 & $\bar{B}$ & \begin{tabular}{|l|}
$01 / 01 / 99$ \\
\end{tabular} & RB72-454 & $20 . \mathrm{C}$ & 2,5 & 2 & 28 & 10 & 63 & $\overline{0,42}$ & 0,08 & 0,14 & 0,07 & 1,00 & 9,33 & & & \\
\hline ST.ROSA II & LE2+LE3 & 9 & 77 & A & 01/01/99 & RB72-454 & $20 . C$ & 3,3 & 3 & 25 & 12 & 51 & 0,55 & 0,13 & 0,13 & $\begin{array}{l}0,09 \\
\end{array}$ & 1,00 & 10,08 & 19,09 & 84,37 & 134,17 \\
\hline ST.ROSA II & LE2+LE3 & 9 & 77 & B & \begin{tabular}{|l|}
$01 / 01 / 99$ \\
\end{tabular} & RB72-454 & $20 . C$ & 2,1 & 2 & 20 & 10 & 63 & 0,35 & 0,08 & 0,10 & 0,07 & 1,00 & \begin{tabular}{|l|}
9,01 \\
\end{tabular} & & & \\
\hline \begin{tabular}{|l|} 
SANTANA \\
\end{tabular} & $\begin{array}{l}\text { LE2+LE3 - } \\
\text { LE3+PE }\end{array}$ & 25 & 23 & A & \begin{tabular}{|l|}
$20 / 10 / 99$ \\
\end{tabular} & $\begin{array}{l}\text { RB72-454 } \\
\end{array}$ & $50 . \mathrm{C}$ & 5,1 & \begin{tabular}{|l|l|}
3 \\
\end{tabular} & 35 & 54 & 50 & 0,85 & 0,13 & 0,18 & 0,58 & 1,00 & \begin{tabular}{|l|}
9,77 \\
\end{tabular} & $\mid 16,94$ & 82,56 & 60,59 \\
\hline SANTANA & $\begin{array}{l}\text { LE2+LE3 - } \\
\text { LE3+PE }\end{array}$ & 25 & 23 & B & $20 / 10 / 99$ & RB72-454 & $50 . \mathrm{C}$ & 3,2 & \begin{tabular}{|l|}
3 \\
\end{tabular} & 23 & 34 & 63 & 0,53 & $0,0,13$ & 0,12 & 0,24 & \begin{tabular}{|l|}
1,00 \\
\end{tabular} & 7,17 & & & \\
\hline SANTANA & $\begin{array}{l}\text { LE2+LE3- } \\
\text { LE3+PE }\end{array}$ & 25 & 24 & A & \begin{tabular}{|l|}
$20 / 10 / 99$ \\
\end{tabular} & $\begin{array}{l}\text { RB83-5089 } \\
\end{array}$ & $50 . \mathrm{C}$ & 4,5 & \begin{tabular}{|l|l|}
6 & \\
\end{tabular} & 134 & 46 & 50 & 0,75 & 0,25 & 1,00 & 1,10 & $1,00 \mid$ & 14,40 & 21,84 & 86,68 & 40,76 \\
\hline SANTANA & $\begin{array}{l}\text { LE2+LE3- } \\
\text { LE3+PE }\end{array}$ & 25 & 24 & B & \begin{tabular}{|l|}
$20 / 10 / 99$ \\
\end{tabular} & RB83-5089 & $50 . C$ & 2,7 & 4 & 44 & 13 & 63 & 0,45 & \begin{tabular}{|l|}
0,17 \\
\end{tabular} & 0,22 & 0,09 & 1,00 & \begin{tabular}{|l|}
7,44 \\
\end{tabular} & & & \\
\hline $\begin{array}{l}\text { SANTANA } \\
\end{array}$ & $\begin{array}{l}\text { LE2+LE3- } \\
\text { LE3+PE }\end{array}$ & 25 & 25 & A & \begin{tabular}{|l|}
$20 / 10 / 99$ \\
\end{tabular} & RB83-5089 & $50 . \mathrm{C}$ & 4,5 & \begin{tabular}{|l|l}
6 & \\
\end{tabular} & 45 & 24 & 50 & 0,75 & 0,25 & 0,23 & 0,17 & 1,00 & \begin{tabular}{|l|}
9,29 \\
\end{tabular} & 16,82 & 82,46 & 40,6 \\
\hline SANTANA & $\begin{array}{l}\text { LE2+LE3 - } \\
\text { LE3+PE }\end{array}$ & 25 & 25 & $\bar{B}$ & \begin{tabular}{|l|}
$20 / 10 / 99$ \\
\end{tabular} & RB83-5089 & $50 . C$ & 2,7 & \begin{tabular}{|c|}
4 \\
\end{tabular} & 49 & 14 & 63 & 0,45 & \begin{tabular}{|l|}
0,17 \\
\end{tabular} & 0,25 & 0,10 & 1,00 & 7,53 & & & \\
\hline SANTANA & $\begin{array}{l}\text { LE2+LE3 - } \\
\text { LE3+PE }\end{array}$ & 25 & 26 & A & \begin{tabular}{|l|}
$20 / 10 / 99$ \\
\end{tabular} & RB72-454 & $50 . \mathrm{C}$ & 5,1 & 3 & 129 & 15 & 50 & 0,85 & 0,13 & 1,00 & 0,11 & 1,00 & 10,82 & 18,20 & 83,62 & 50,4 \\
\hline SANTANA & $\begin{array}{l}\text { LE2+LE3- } \\
\text { LE3+PE }\end{array}$ & 25 & 26 & B & \begin{tabular}{|l|}
$20 / 10 / 99$ \\
\end{tabular} & RB72-454 & $50 . \mathrm{C}$ & 3,2 & \begin{tabular}{|l|l|}
3 & \\
\end{tabular} & 47 & 27 & 63 & 0,53 & \begin{tabular}{|l|}
0,13 \\
\end{tabular} & 0,24 & 0,19 & 1,00 & 7,38 & & & \\
\hline SANTANA & $\begin{array}{l}\text { LE2+LE3- } \\
\text { LE3+PE }\end{array}$ & 28 & 95 & A & 01/01/99 & 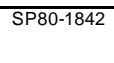 & $20 . \mathrm{C}$ & \begin{tabular}{|l|}
10,9 \\
\end{tabular} & \begin{tabular}{|l|}
36 \\
\end{tabular} & 103 & 63 & 50 & 1,00 & 1,00 & 0,78 & 0,76 & \begin{tabular}{|l|}
1,00 \\
\end{tabular} & 18,12 & 35,50 & 98,17 & $\overline{143,0}$ \\
\hline SANTANA & $\begin{array}{l}\text { LE2+LE3- } \\
\text { LE3+PE }\end{array}$ & 28 & 95 & B & |01/01/99 & SP80-1842 & $20 . C$ & \begin{tabular}{|l|}
11,5 \\
\end{tabular} & 12 & 119 & 73 & 63 & 1,00 & 0,42 & 0,94 & 0,96 & 1,00 & 17,38 & & & \\
\hline SANTANA & $\begin{array}{l}\text { LE2+LE3 - } \\
\text { LE3+PE }\end{array}$ & 28 & 97 & A & \begin{tabular}{|l|}
$01 / 01 / 99$ \\
\end{tabular} & $\begin{array}{l}\text { SP80-1842 } \\
\end{array}$ & $20 . C$ & 9,4 & 39 & 109 & 56 & 50 & 1,00 & 1,00 & 0,84 & 0,62 & \begin{tabular}{|l|}
1,00 \\
\end{tabular} & 17,73 & 33,86 & 96,79 & 127,8 \\
\hline SANTANA & $\begin{array}{l}\text { LE2+LE3- } \\
\text { LE3+PE }\end{array}$ & 28 & 97 & B & |01/01/99 & SP80-1842 & $20 . C$ & 8,9 & \begin{tabular}{l|l}
6 & \\
\end{tabular} & 113 & \begin{tabular}{l|}
66 \\
\end{tabular} & $\begin{array}{ll}63 \\
\end{array}$ & 1,00 & 0,25 & 0,88 & 0,82 & 1,00 & 16,13 & & & \\
\hline SANTANA & $\begin{array}{l}\text { LE2+LE3 - } \\
\text { LE3+PE }\end{array}$ & 28 & 98 & A & $01 / 01 / 99$ & SP80-1842 & $20 . \mathrm{C}$ & 9,6 & 32 & 102 & 53 & 50 & 1,00 & \begin{tabular}{|l|}
0,93 \\
\end{tabular} & 0,77 & 0,56 & 1,00 & 17,27 & 33,16 & 96,21 & 73,4 \\
\hline SANTANA & $\begin{array}{l}\text { LE2+LE3- } \\
\text { LE3+PE }\end{array}$ & 28 & 98 & B & \begin{tabular}{|l|}
$01 / 01 / 99$ \\
\end{tabular} & SP80-1842 & $20 . C$ & 9,5 & \begin{tabular}{|l|}
11 \\
\end{tabular} & 119 & 56 & 63 & 1,00 & 0,39 & 0,94 & 0,62 & 1,00 & 15,90 & & & \\
\hline SANTANA & $\begin{array}{l}\text { LE2+LE3- } \\
\text { LE3+PE }\end{array}$ & 28 & 104 & A & \begin{tabular}{|l|}
$01 / 01 / 99$ \\
\end{tabular} & SP80-1842 & $20 . \mathrm{C}$ & 11,0 & 92 & 115 & 63 & 50 & 1,00 & 1,00 & 0,90 & 0,76 & \begin{tabular}{|l|}
1,00 \\
\end{tabular} & 18,93 & 37,67 & 100,00 & 118,20 \\
\hline SANTANA & $\begin{array}{l}\text { LE2+LE3- } \\
\text { LE3+PE }\end{array}$ & 28 & 104 & B & \begin{tabular}{|l|}
$01 / 01 / 99$ \\
\end{tabular} & $\begin{array}{l}\text { SP80-1842 } \\
\text { (1) }\end{array}$ & $20 . C$ & \begin{tabular}{|l|}
10,8 \\
\end{tabular} & 32 & 97 & 46 & 63 & 1,00 & \begin{tabular}{|l|l}
0,93 \\
\end{tabular} & 0,72 & \begin{tabular}{|l|}
1,10 \\
\end{tabular} & 1,00 & $\mid 18,74$ & & & \\
\hline \begin{tabular}{|l} 
STA. LIDIA \\
\end{tabular} & $\mathrm{LI} / 3$ & 36 & 17 & A & 01/01/99 & $\begin{array}{l}\text { RB82-5336 } \\
\end{array}$ & $20 . C$ & 8,9 & \begin{tabular}{|l|}
113 \\
\end{tabular} & 48 & 47 & 33 & 1,00 & 1,00 & 0,24 & 1,12 & \begin{tabular}{|l|}
0,66 \\
\end{tabular} & 16,55 & 30,63 & 94,08 & 54,92 \\
\hline \begin{tabular}{|l} 
STA. LIDIA \\
\end{tabular} & $\mathrm{L} / 3$ & 36 & 17 & B & $01 / 01 / 99$ & RB82-5336 & $20 . \mathrm{C}$ & 3,1 & 42 & 40 & 37 & 35 & 0,52 & 1,00 & 0,20 & 0,95 & 0,70 & 14,08 & & & \\
\hline PITANGA & $\mathrm{L} 13+\mathrm{L} / 2$ & 20 & 48 & A & \begin{tabular}{|l|}
$20 / 10 / 99$ \\
\end{tabular} & RB82-5336 & $20 . \mathrm{C}$ & 0,8 & 24 & 129 & 50 & 29 & 0,13 & $0,0,73$ & 1,00 & 0,50 & 0,58 & 12,94 & 24,18 & 88,65 & 51,36 \\
\hline PITANGA & $\mathrm{L} 13+\mathrm{LI} 2$ & 20 & 48 & B & 20/10/99 & $\begin{array}{l}\text { RB82-5336 } \\
\end{array}$ & $20 . \mathrm{C}$ & 0,8 & \begin{tabular}{|l|}
18 \\
\end{tabular} & 205 & 9 & 35 & 0,13 & \begin{tabular}{|l|}
0,58 \\
\end{tabular} & 1,00 & 0,06 & 0,70 & 11,24 & & & \\
\hline PITANGA & $\mathrm{LI} 3+\mathrm{LI} 2$ & 16 & $11 \mathrm{a}$ & A & 01/01/99 & RB78-5148 & $60 . C$ & 1,0 & \begin{tabular}{l|l}
10 \\
\end{tabular} & 91 & \begin{tabular}{l|l}
42 \\
\end{tabular} & 29 & 0,17 & 0,36 & 0,66 & 1,03 & 0,58 & 7,96 & 13,41 & 79,59 & 42,29 \\
\hline PITANGA & $\mathrm{L} 13+\mathrm{LI} 2$ & 16 & $11 \mathrm{a}$ & B & |01/01/99 & $\begin{array}{l}\text { RB78-5148 } \\
\end{array}$ & $60 . \mathrm{C}$ & 1,2 & \begin{tabular}{|l|l|}
3 & \\
\end{tabular} & 110 & 27 & 35 & 0,20 & 0,13 & 0,85 & 0,19 & 0 & \begin{tabular}{|l|}
5,45 \\
\end{tabular} & & & \\
\hline \begin{tabular}{|l} 
STA. MARIA \\
\end{tabular} & $\mathrm{LI} 3+\mathrm{LI} 2$ & 32 & 1 & $\bar{A}$ & \begin{tabular}{|l|}
$10 / 09 / 99$ \\
\end{tabular} & RB76-5418 & $50 . \mathrm{C}$ & 27,0 & 87 & 25 & 28 & 29 & 1,00 & 1,00 & 0,13 & 0,20 & 0,58 & \begin{tabular}{|l|}
9,99 \\
\end{tabular} & $\mid 19,89$ & 85,04 & 60,69 \\
\hline STA. MARIA & $\mathrm{LI} 3+\mathrm{LI} 2$ & 32 & 1 & B & \begin{tabular}{|l|}
$10 / 09 / 99$ \\
\end{tabular} & RB76-5418 & $50 . \mathrm{C}$ & $\begin{array}{ll}11,6 \\
\end{array}$ & 39 & 22 & 27 & 35 & 1,00 & 1,00 & 0,11 & 0,19 & 0,70 & 9,91 & & & \\
\hline STA. MARIA & $L 13+\mathrm{LI} 2$ & 32 & 5 & A & \begin{tabular}{|l|}
$10 / 09 / 99$ \\
\end{tabular} & RB76-5418 & $50 . C$ & \begin{tabular}{|l|}
7,3 \\
\end{tabular} & 60 & 26 & 23 & 29 & 1,00 & 1,00 & 0,13 & 0,16 & 0,58 & 10,87 & 21,56 & 86,45 & 56,30 \\
\hline \begin{tabular}{|l|} 
STA. MARIA \\
\end{tabular} & $L 13+L 12$ & 32 & 5 & $\bar{B}$ & \begin{tabular}{|l|}
$10 / 09 / 99$ \\
\end{tabular} & $\begin{array}{l}\text { RB76-5418 } \\
\end{array}$ & $50 . \mathrm{C}$ & 7,6 & 60 & 30 & 16 & 35 & 1,00 & 1,00 & 0,15 & 0,11 & 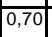 & 10,69 & & & \\
\hline STA. MARIA & $L 13+\mathrm{LI} 2$ & 32 & 21 & A & $01 / 01 / 99$ & RB76-5418 & $60 . C$ & 8,8 & 18 & 34 & 41 & 29 & 1,00 & 0,58 & 0,17 & 1,02 & \begin{tabular}{|c|}
0,58 \\
\end{tabular} & 10,18 & 18,61 & 83,96 & 80,47 \\
\hline STA. MARIA & $\mathrm{LI} 3+\mathrm{LI} 2$ & 32 & 21 & B & |01/01/99 & RB76-5418 & $60 . \mathrm{C}$ & 8,8 & \begin{tabular}{|l|}
11 \\
\end{tabular} & 29 & 55 & 35 & 1,00 & 0,39 & 0,15 & 0,60 & 0,70 & 8,43 & & & \\
\hline \begin{tabular}{|l|} 
S.BENEDITO \\
\end{tabular} & $\mathrm{LI} 3+\mathrm{PV} 9$ & 3 & 6 & A & \begin{tabular}{|l|}
$04 / 08 / 99$ \\
\end{tabular} & RB76-5418 & $40 . C$ & 1,6 & \begin{tabular}{|l|l|}
6 & \\
\end{tabular} & 88 & 52 & 30 & 0,27 & 0,25 & 0,63 & 0,54 & | 0,60 & \begin{tabular}{|l|}
8,81 \\
\end{tabular} & $\mid 18,61$ & 83,97 & 59,44 \\
\hline \begin{tabular}{|l|} 
S.BENEDITO \\
\end{tabular} & LI3+PV9 & 3 & 6 & B & \begin{tabular}{|l|}
$04 / 08 / 99$ \\
\end{tabular} & RB76-5418 & $40 . C$ & 1,0 & \begin{tabular}{|c|}
13 \\
\end{tabular} & 85 & 50 & 49 & 0,17 & 0,44 & 0,60 & 0,50 & \begin{tabular}{|l|}
0,98 \\
\end{tabular} & \begin{tabular}{|l|}
9,80 \\
\end{tabular} & & & \\
\hline S.BENEDITO & LI3+PV9 & 3 & 8 & A & 04/08/99 & RB76-5418 & $40 . C$ & 2,3 & \begin{tabular}{|l|l|}
5 & \\
\end{tabular} & 94 & 23 & 30 & 0,38 & 0,21 & 0,69 & 0,16 & \begin{tabular}{|l|l|}
0,60 \\
\end{tabular} & \begin{tabular}{|l|}
8,24 \\
\end{tabular} & $\mid 16,24$ & 81,97 & 59,90 \\
\hline
\end{tabular}




\begin{tabular}{|c|c|c|c|c|c|c|c|c|c|c|c|c|c|c|c|c|c|c|c|c|c|}
\hline nome & & & & & & & & teores & & & & & pontuação & & & & & & & & \\
\hline Fazenda & Solo & \begin{tabular}{|l|} 
zona \\
\end{tabular} & talhão & horiz & coleta & Variedade & Estágio & $\mathrm{K}$ & $\mathrm{P}$ & & $\mathrm{v}$ & Arg & $\mathrm{K}$ & $P$ & $T$ & $\mathrm{v}$ & \begin{tabular}{|l|} 
Arg \\
\end{tabular} & $\mathrm{NP}$ & Ps & od estima & od medida \\
\hline S.BENEDITO & $\mathrm{L13+PV9}$ & 3 & 8 & B & \begin{tabular}{|l|}
$04 / 08 / 99$ \\
\end{tabular} & RB76-5418 & $40 . C$ & 1,0 & 3 & 77 & 32 & 49 & 0,17 & 0,13 & 0,52 & 0,23 & 0,98 & 8,01 & & & \\
\hline S.BENEDITO & $13+\mathrm{PV9}$ & 3 & 9 & A & $4 / 7 / 989$ & $\begin{array}{l}\text { RB76-5418 } \\
\end{array}$ & $40 . \mathrm{C}$ & 1,6 & 6 & 52 & 61 & 30 & 0,27 & 0,25 & 0,77 & 0,72 & 0,60 & \begin{tabular}{|l|}
9,77 \\
\end{tabular} & \begin{tabular}{|l|}
20,54 \\
\end{tabular} & 85,59 & 77,1 \\
\hline S.BENEDITO & $\mathrm{L} 13+\mathrm{PV} 9$ & 3 & 9 & B & $4 / 08 / 99$ & RB76-5418 & $40 . \mathrm{C}$ & 0,8 & 8 & 52 & 65 & 49 & $\overline{0,13}$ & 0,31 & $\mid 0,77$ & 0,80 & $\mid 0,98$ & \begin{tabular}{|l|}
10,77 \\
\end{tabular} & & & \\
\hline S.BENEDITO & $\mathrm{LI} 3+\mathrm{PV} 9$ & 3 & 11 & $\bar{A}$ & \begin{tabular}{|l|}
$04 / 08 / 99$ \\
\end{tabular} & RB76-5418 & $40 . C$ & 1,6 & 6 & 120 & 79 & 30 & 0,27 & 0,25 & 0,95 & 1,00 & 0,60 & $\mid 11,30$ & $\mid 22,58$ & 87,30 & 79,5 \\
\hline S.BENEDITO & LI3+PV9 & 3 & 11 & $\bar{B}$ & \begin{tabular}{|l|}
$04 / 08 / 99$ \\
\end{tabular} & RB76-5418 & $40 . C$ & 0,8 & 3 & 124 & \begin{tabular}{|l|}
69 \\
\end{tabular} & 49 & 0,13 & 0,13 & 0,99 & 0,88 & 0,98 & \begin{tabular}{|l|}
11,28 \\
\end{tabular} & & & \\
\hline S.BENEDITO & $\mathrm{LI} 3+\mathrm{PV} 9$ & 3 & 13 & $\bar{A}$ & \begin{tabular}{|l|}
$04 / 08 / 99$ \\
\end{tabular} & RB76-5418 & $40 . C$ & 1,6 & 10 & 108 & 51 & 30 & 0,27 & 0,36 & 0,83 & 0,52 & 0,60 & 9,46 & \begin{tabular}{|l|}
21,17 \\
\end{tabular} & 86,12 & 40,77 \\
\hline S.BENEDITO & $\mathrm{L} 13+\mathrm{PV} 9$ & 3 & 13 & B & $4 / 08 / 99$ & RB76-5418 & $40 . \mathrm{C}$ & 1,3 & 14 & 142 & 63 & 49 & $\overline{0,22}$ & 0,47 & 1,00 & $\mid 0,76$ & 0,98 & $\mid 11,71$ & & & \\
\hline S.BENEDITO & LI3+PV9 & 3 & 14 & $\bar{A}$ & \begin{tabular}{|l|}
$04 / 08 / 99$ \\
\end{tabular} & $\begin{array}{l}\text { RB76-5418 } \\
\end{array}$ & $40 . \mathrm{C}$ & 1,6 & 10 & 118 & 83 & 30 & 0,27 & 0,36 & 0,93 & 1,00 & 0,60 & 11,20 & 23,21 & 87,84 & 56,1 \\
\hline S.BENEDITO & LI3+PV9 & 3 & 14 & $B$ & \begin{tabular}{|l|}
$04 / 08 / 99$ \\
\end{tabular} & RB76-5418 & $40 . \mathrm{C}$ & 1,3 & 14 & 132 & 68 & 49 & 0,22 & 0,47 & 1,00 & 0,86 & 0,98 & \begin{tabular}{|l|}
12,01 \\
\end{tabular} & & & \\
\hline S.BENEDITO & $\mathrm{LI} 3+\mathrm{PV} 9$ & 5 & 28 & $\bar{A}$ & \begin{tabular}{|l|}
$01 / 01 / 99$ \\
\end{tabular} & RB72-454 & $50 . \mathrm{C}$ & 8,7 & 20 & 132 & 68 & 30 & 1,00 & 0,63 & 1,00 & 0,86 & 0,60 & $\mid 13,16$ & 25,33 & 89,62 & 56,96 \\
\hline S.BENEDITO & LI3+PV9 & 5 & 28 & B & \begin{tabular}{|l|}
$01 / 01 / 99$ \\
\end{tabular} & RB72-454 & $50 . \mathrm{C}$ & 4,4 & 13 & 105 & 70 & 49 & 0,73 & 0,44 & 0,80 & 0,90 & 0,98 & $|12,17|$ & & & \\
\hline S.BENEDITO & $\mathrm{L} 13+\mathrm{PV} 9$ & 5 & 30 & $\bar{A}$ & \begin{tabular}{|l|}
$04 / 08 / 99$ \\
\end{tabular} & RB76-5418 & $50 . \mathrm{C}$ & 0,6 & 8 & 118 & 76 & 30 & 0,10 & 0,31 & 0,93 & 1,00 & 0,60 & \begin{tabular}{|l|}
9,78 \\
\end{tabular} & \begin{tabular}{|l|}
20,17 \\
\end{tabular} & 85,28 & 50,7 \\
\hline S.BENEDITO & LI3+PV9 & 5 & 30 & $\bar{B}$ & \begin{tabular}{|l|}
$04 / 08 / 99$ \\
\end{tabular} & $\begin{array}{l}\text { RB76-5418 } \\
\end{array}$ & $50 . \mathrm{C}$ & $\overline{0,6}$ & 4 & 119 & 76 & 49 & 0,10 & 0,17 & 0,94 & 1,00 & 0,98 & $\mid 10,39$ & & & \\
\hline S.BENEDITO & LI3+PV9 & 5 & 32 & $\bar{A}$ & \begin{tabular}{|l|}
$04 / 08 / 99$ \\
\end{tabular} & $\begin{array}{l}\text { RB76-5418 } \\
\end{array}$ & $50 . \mathrm{C}$ & 1,3 & 9 & 143 & 76 & 30 & 0,22 & 0,33 & 1,00 & 1,00 & 0,60 & 10,50 & $\mid 19,68$ & 84,86 & 45,8 \\
\hline S.BENEDITO & $\mathrm{L} 13+\mathrm{PV} 9$ & 5 & 32 & B & \begin{tabular}{|l|}
$04 / 08 / 99$ \\
\end{tabular} & RB76-5418 & $50 . \mathrm{C}$ & 0,8 & 3 & 131 & 51 & 49 & 0,13 & 0,13 & 1,00 & 0,52 & 0,98 & \begin{tabular}{|l|}
9,18 \\
\end{tabular} & & & \\
\hline S.BENEDITO & LI3+PV9 & 5 & $31 a$ & A & \begin{tabular}{|l|}
$01 / 01 / 99$ \\
\end{tabular} & RB76-5418 & $50 . \mathrm{C}$ & 2,8 & 15 & 88 & 71 & 30 & 0,47 & 0,50 & 0,63 & 0,92 & 0,60 & $\mid 10,33$ & 18,34 & 83,74 & 50,2 \\
\hline S.BENEDITO & L13+PV9 & 5 & $31 a$ & $\bar{B}$ & \begin{tabular}{|l|}
$01 / 01 / 99$ \\
\end{tabular} & RB76-5418 & $50 . \mathrm{C}$ & 2,0 & 5 & 48 & 58 & 49 & 0,33 & 0,21 & 0,24 & 0,66 & 0,98 & \begin{tabular}{|l|}
8,02 \\
\end{tabular} & & & \\
\hline S.BENEDITO & $\mathrm{L} 13+\mathrm{PV} 9$ & 5 & $33 a$ & A & \begin{tabular}{|l|}
$01 / 01 / 99$ \\
\end{tabular} & $\begin{array}{l}\text { RB76-5418 } \\
\end{array}$ & $40 . \mathrm{C}$ & 8,7 & 20 & 157 & \begin{tabular}{|c|}
63 \\
\end{tabular} & 30 & 1,00 & 0,63 & 1,00 & 0,76 & 0,60 & $\mid 13,83$ & 27,88 & 91,76 & 46,79 \\
\hline S.BENEDITO & $\mathrm{L} 13+\mathrm{PV} 9$ & 5 & $33 a$ & B & \begin{tabular}{|l|}
$01 / 01 / 99$ \\
\end{tabular} & RB76-5418 & $40 . \mathrm{C}$ & 4,4 & 13 & 147 & 78 & 49 & 0,73 & 0,44 & 1,00 & 1,00 & $\mid 0,98$ & $\mid 14,05$ & & & \\
\hline BOA VISTA & PE-PV9 & 50 & 18 & $\bar{A}$ & \begin{tabular}{|l|}
$20 / 10 / 99$ \\
\end{tabular} & SP70-1143 & $60 \mathrm{C}$ & 1,6 & 4 & 68 & 11 & 30 & 0,27 & 0,17 & 0,93 & 0,08 & 0,60 & \begin{tabular}{|l|}
6,95 \\
\end{tabular} & 12,94 & 79,19 & 80,8 \\
\hline \begin{tabular}{|l} 
BOA VISTA \\
\end{tabular} & PE-PV9 & 50 & 18 & $\bar{B}$ & \begin{tabular}{|l|}
$20 / 10 / 99$ \\
\end{tabular} & SP70-1143 & $60 \mathrm{C}$ & 0,6 & 3 & 46 & 52 & 45 & 0,10 & 0,13 & 0,23 & 0,54 & 0,90 & \begin{tabular}{|l|}
5,99 \\
\end{tabular} & & & \\
\hline BOA VISTA & PE-PV9 & 50 & 19 & A & \begin{tabular}{|l|}
$20 / 10 / 99$ \\
\end{tabular} & RB72-454 & $50 \mathrm{C}$ & 1,6 & 4 & 200 & 12 & 30 & 0,27 & $\mid 0,17$ & 1,00 & 0,09 & 0,60 & \begin{tabular}{|l|}
8,16 \\
\end{tabular} & 16,00 & 81,77 & 96,10 \\
\hline BOA VISTA & PE-PV9 & 50 & 19 & B & \begin{tabular}{|l|}
$20 / 10 / 99$ \\
\end{tabular} & RB72-454 & $50 \mathrm{C}$ & 0,6 & 3 & 205 & \begin{tabular}{|l|}
9 \\
\end{tabular} & 45 & 0,10 & 0,13 & 1,00 & 0,06 & 0,90 & \begin{tabular}{|l|}
7,84 \\
\end{tabular} & & & \\
\hline BOA VISTA & PE-PV9 & 50 & 20 & $\bar{A}$ & \begin{tabular}{|l|}
$20 / 10 / 99$ \\
\end{tabular} & RB72-454 & $50 \mathrm{C}$ & 2,1 & 10 & 46 & $\mid 17$ & 30 & 0,35 & 0,36 & 0,23 & 0,12 & 0,60 & \begin{tabular}{|l|}
6,56 \\
\end{tabular} & 12,02 & 78,42 & 123,09 \\
\hline \begin{tabular}{|l} 
BOA VISTA \\
\end{tabular} & PE-PV9 & 50 & 20 & $\bar{B}$ & \begin{tabular}{|l|}
$20 / 10 / 99$ \\
\end{tabular} & RB72-454 & $50 \mathrm{C}$ & 0,8 & 3 & 39 & 20 & 45 & 0,13 & 0,13 & 0,20 & 0,14 & 0,90 & 5,46 & & & \\
\hline BOA VISTA & PE - PV9 & 50 & 21 & A & 20/10/99 & $\begin{array}{l}\text { RB72-454 } \\
\end{array}$ & $50 \mathrm{C}$ & 1,3 & \begin{tabular}{|l|l|}
4 \\
\end{tabular} & 46 & 26 & 30 & 0,22 & 0,17 & 0,23 & 0,18 & 0,60 & \begin{tabular}{|l|}
5,54 \\
\end{tabular} & $|12,62|$ & 78,93 & 82,26 \\
\hline BOA VISTA & PE-PV9 & 50 & 21 & $\bar{B}$ & \begin{tabular}{|l|}
$20 / 10 / 99$ \\
\end{tabular} & RB72-454 & $50 \mathrm{C}$ & 0,6 & 3 & 53 & 11 & 45 & 0,10 & 0,13 & 0,78 & 0,08 & 0,90 & \begin{tabular}{|l|}
7,07 \\
\end{tabular} & & & \\
\hline BOA VISTA & PE-PV9 & 50 & 27 & A & \begin{tabular}{|l|}
$20 / 10 / 99$ \\
\end{tabular} & RB78-5148 & $50 \mathrm{C}$ & 6,2 & 28 & 51 & 25 & 30 & 1,00 & 0,83 & 0,76 & 0,18 & 0,60 & \begin{tabular}{|l|}
11,36 \\
\end{tabular} & 21,97 & 86,79 & 60,68 \\
\hline BOA VISTA & PE - PV9 & 50 & 27 & $B$ & \begin{tabular}{|l|}
$20 / 10 / 99$ \\
\end{tabular} & RB78-5148 & $50 \mathrm{C}$ & 3,0 & 13 & 48 & 93 & 45 & 0,50 & 0,44 & 0,24 & 1,00 & 0,90 & $|10,60|$ & & & \\
\hline \begin{tabular}{|l} 
BOA VISTA \\
\end{tabular} & PE-PV9 & 50 & 28 & A & \begin{tabular}{|l|}
$20 / 10 / 99$ \\
\end{tabular} & $\begin{array}{l}\text { RB78-5148 } \\
\end{array}$ & $50 \mathrm{C}$ & 4,4 & 8 & 72 & 41 & 30 & 0,73 & 0,31 & $\mid 0,97$ & 1,02 & 0,60 & 13,13 & 20,96 & 85,95 & 66,80 \\
\hline \begin{tabular}{|l} 
BOA VISTA \\
\end{tabular} & PE-PV9 & 50 & 28 & B & \begin{tabular}{|l|}
$20 / 10 / 99$ \\
\end{tabular} & $\begin{array}{l}\text { RB78-5148 } \\
\end{array}$ & $50 \mathrm{C}$ & 1,3 & 4 & 50 & 32 & 45 & 0,22 & 0,17 & 0,75 & 0,23 & 0,90 & \begin{tabular}{|l|}
7,83 \\
\end{tabular} & & & \\
\hline BOA VISTA & PE-PV9 & 50 & 32 & $\bar{A}$ & \begin{tabular}{|l|}
$20 / 10 / 99$ \\
\end{tabular} & SP70-1143 & $80 \mathrm{C}$ & 4,4 & 8 & 127 & 49 & 30 & 0,73 & 0,31 & 1,00 & 1,15 & 0,60 & 10,70 & 16,20 & 81,94 & 61,67 \\
\hline \begin{tabular}{|l|} 
BOA VISTA \\
\end{tabular} & PE - PV9 & 50 & 32 & $B$ & \begin{tabular}{|l|}
$20 / 10 / 99$ \\
\end{tabular} & SP70-1143 & $80 \mathrm{C}$ & 1,3 & 4 & 201 & 25 & 45 & 0,22 & 0,17 & 1,00 & 0,18 & 30,90 & \begin{tabular}{|l|}
5,51 \\
\end{tabular} & & & \\
\hline BOA VISTA & PE-PV9 & 50 & 33 & $\bar{A}$ & \begin{tabular}{|l|}
$24 / 02 / 99$ \\
\end{tabular} & RB78-5148 & $50 \mathrm{C}$ & 3,8 & 18 & 22 & 5 & 30 & 0,63 & 0,58 & 0,11 & 0,68 & 0,60 & \begin{tabular}{|l|}
9,00 \\
\end{tabular} & $|18,65|$ & 84,00 & 45,21 \\
\hline BOA VISTA & PE-PV9 & 50 & 33 & $\mathrm{~B}$ & \begin{tabular}{|l|}
$24 / 02 / 99$ \\
\end{tabular} & $\begin{array}{l}\text { RB78-5148 } \\
\end{array}$ & $50 \mathrm{C}$ & 2,0 & 11 & 71 & 33 & 45 & 0,33 & 0,39 & $\mid 0,96$ & 0,23 & 0,90 & \begin{tabular}{|l|}
9,65 \\
\end{tabular} & & & \\
\hline COVETINGA & PV7 & 20 & 2 & $\bar{A}$ & \begin{tabular}{|l|}
$01 / 01 / 99$ \\
\end{tabular} & RB72-454 & $20 . \mathrm{C}$ & 6,8 & 4 & 98 & \begin{tabular}{|l|}
26 \\
\end{tabular} & & 1,00 & 0,17 & 0,73 & 0,18 & 30,00 & $\mid$\begin{tabular}{|l|}
10,97 \\
\end{tabular} & 21,33 & 86,25 & 68,42 \\
\hline \begin{tabular}{|c|} 
COVETINGA \\
\end{tabular} & PV7 & 20 & 2 & $\bar{B}$ & \begin{tabular}{|l|}
$01 / 01 / 99$ \\
\end{tabular} & RB72-454 & $20 . C$ & 4,8 & 3 & 137 & 11 & & 0,80 & 0,13 & 1,00 & 0,08 & 30,00 & $\mid 10,36$ & & & \\
\hline \begin{tabular}{|c|} 
COVETINGA \\
\end{tabular} & PV7 & 20 & 4 & A & \begin{tabular}{|l|}
$01 / 01 / 99$ \\
\end{tabular} & RB72-454 & $20 . C$ & 3,2 & 3 & 66 & 42 & & 0,53 & 0,13 & 0,91 & 1,03 & 0,00 & 12,31 & 23,76 & 88,30 & 81,78 \\
\hline \begin{tabular}{|c|} 
COVETINGA \\
\end{tabular} & PV7 & 20 & 4 & $\bar{B}$ & \begin{tabular}{|l|}
$01 / 01 / 99$ \\
\end{tabular} & RB72-454 & $20 . \mathrm{C}$ & 2,6 & 4 & 94 & 49 & & 0,43 & 0,17 & 0,69 & 1,15 & 0,00 & \begin{tabular}{|l|}
11,45 \\
\end{tabular} & & & \\
\hline COVETINGA & PV7 & 20 & 12 & $\bar{A}$ & \begin{tabular}{|l|}
$01 / 01 / 99$ \\
\end{tabular} & RB72-454 & $20 . C$ & 2,6 & 6 & 84 & 49 & & 0,43 & 0,25 & 0,59 & $\mid 1,15$ & 0,00 & 12,00 & $\mid 23,89$ & 88,41 & 78,0 \\
\hline COVETINGA & PV7 & 20 & 12 & $\mathrm{~B}$ & \begin{tabular}{|l|}
$01 / 01 / 99$ \\
\end{tabular} & RB72-454 & $20 . C$ & 2,4 & 3 & 73 & 42 & & 0,40 & 0,13 & 0,98 & $\mid 1,03$ & 0,00 & 11,89 & & & \\
\hline COVETINGA & PV7 & 18 & $5 a$ & $\bar{A}$ & \begin{tabular}{|l|}
$01 / 01 / 99$ \\
\end{tabular} & RB72-454 & $20 . \mathrm{C}$ & 2,6 & 4 & 82 & 58 & & 0,43 & 0,17 & 0,57 & 0,66 & 0,00 & \begin{tabular}{|l|}
9,84 \\
\end{tabular} & $\mid 18,15$ & 83,58 & $\overline{100,20}$ \\
\hline \begin{tabular}{|c|} 
COVETINGA \\
\end{tabular} & PV7 & 18 & $5 a$ & B & 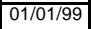 & RB72-454 & $20 . \mathrm{C}$ & 1,3 & 2 & 71 & 26 & & 0,22 & 0,08 & 0,96 & 0,18 & 0,00 & 8,31 & & & \\
\hline COVETINGA & PV7 & 18 & $6 \mathrm{a}$ & $\bar{A}$ & \begin{tabular}{|l|}
$01 / 01 / 99$ \\
\end{tabular} & RB72-454 & $20 . \mathrm{C}$ & 1,3 & 5 & 171 & \begin{tabular}{|l|}
62 \\
\end{tabular} & & 0,22 & 0,21 & 1,00 & 0,74 & 0,00 & \begin{tabular}{|l|}
10,55 \\
\end{tabular} & 18,39 & 83,78 & 76,86 \\
\hline COVETINGA & PV7 & 18 & $6 \mathrm{a}$ & $\bar{B}$ & \begin{tabular}{|l|}
$01 / 01 / 99$ \\
\end{tabular} & RB72-454 & $20 . \mathrm{C}$ & 0,9 & 6 & 105 & 23 & & 0,15 & 0,25 & 0,80 & 0,16 & 0,00 & \begin{tabular}{|l|}
7,84 \\
\end{tabular} & & & \\
\hline P.VERMELHO & PV7 & 59 & 18 & A & \begin{tabular}{|l|}
$01 / 01 / 99$ \\
\end{tabular} & $\begin{array}{l}\text { RB72-454 } \\
\end{array}$ & $20 . C$ & 2,5 & 3 & 89 & 41 & & 0,42 & 0,13 & 0,64 & 1,02 & 0,00 & 10,50 & 17,35 & 82,91 & 79,64 \\
\hline \begin{tabular}{|l|} 
P.VERMELHO \\
\end{tabular} & PV7 & 59 & 18 & $\bar{B}$ & \begin{tabular}{|l|}
$01 / 01 / 99$ \\
\end{tabular} & RB72-454 & $20 . C$ & 1,9 & 1 & 81 & 20 & & 0,32 & 0,04 & 0,56 & 0,14 & 0,00 & \begin{tabular}{|l|}
6,86 \\
\end{tabular} & & & \\
\hline CANAL TORT & PV9 & 40 & 9 & $\bar{A}$ & \begin{tabular}{|l|}
$11 / 11 / 99$ \\
\end{tabular} & SP81-1763 & $40 . \mathrm{C}$ & 0,8 & 4 & 67 & \begin{tabular}{|l|}
29 \\
\end{tabular} & 21 & 0,13 & 0,17 & 0,92 & 0,21 & 0,42 & $8, \mid$ & $\mid 16,43$ & 82,13 & 85,9 \\
\hline CANAL TORT & PV9 & 40 & 9 & $B$ & \begin{tabular}{|l|}
$11 / 11 / 99$ \\
\end{tabular} & SP81-1763 & $40 . \mathrm{C}$ & 0,3 & 1 & 67 & 29 & 43 & 0,05 & 0,04 & 0,92 & 0,21 & 0,86 & \begin{tabular}{|l|}
8,33 \\
\end{tabular} & & & \\
\hline SAO LUIS & PV9 & 2 & 35 & A & \begin{tabular}{|l|}
$05 / 10 / 99$ \\
\end{tabular} & RB76-5418 & $50 . \mathrm{C}$ & 1,6 & 8 & 77 & 50 & 21 & 0,27 & 0,31 & 0,52 & 0,50 & 0,42 & 7,62 & 18,10 & 83,54 & 65,43 \\
\hline SAO LUIS & PV9 & 2 & 35 & B & \begin{tabular}{|l|}
$05 / 10 / 99$ \\
\end{tabular} & $\begin{array}{l}\text { RB76-5418 } \\
\end{array}$ & $50 . \mathrm{C}$ & 1,0 & 8 & 61 & 37 & 43 & 0,17 & 0,31 & 0,86 & 0,95 & \begin{tabular}{|l|l|}
5,86 \\
\end{tabular} & $\mid 10,48$ & & & \\
\hline SAO LUIS & PV9 & 2 & 36 & A & \begin{tabular}{|l|}
$05 / 10 / 99$ \\
\end{tabular} & RB76-5418 & $50 . \mathrm{C}$ & 1,8 & 13 & 87 & \begin{tabular}{|l|}
56 \\
\end{tabular} & 21 & 0,30 & 0,44 & 0,62 & 0,62 & 0,42 & \begin{tabular}{|l|}
8,87 \\
\end{tabular} & 16,55 & 82,23 & 65,0 \\
\hline SAOLUIS & PV9 & 2 & 36 & $\bar{B}$ & \begin{tabular}{|l|}
$05 / 10 / 99$ \\
\end{tabular} & RB76-5418 & $50 . \mathrm{C}$ & 0,5 & 3 & 87 & 51 & 43 & 0,08 & 0,13 & 0,62 & 0,52 & 0,86 & \begin{tabular}{|l|}
7,68 \\
\end{tabular} & & & \\
\hline SAOLUIS & PV9 & 2 & 38 & $\bar{A}$ & \begin{tabular}{|l|}
$05 / 10 / 99$ \\
\end{tabular} & RB76-5418 & $50 . \mathrm{C}$ & 1,8 & 15 & 66 & 36 & 21 & 0,30 & 0,50 & 0,91 & 0,93 & 30,42 & $\mid$\begin{tabular}{|l|}
10,47 \\
\end{tabular} & 19,51 & 84,72 & 57,41 \\
\hline SAO LUIS & PV9 & 2 & 38 & B & \begin{tabular}{|l|}
$05 / 10 / 99$ \\
\end{tabular} & RB76-5418 & $50 . \mathrm{C}$ & 0,5 & 29 & 99 & 11 & 43 & 0,08 & 0,85 & 0,74 & 0,08 & \begin{tabular}{l|l|}
30,86 \\
\end{tabular} & \begin{tabular}{|l|}
9,03 \\
\end{tabular} & & & \\
\hline SAO LUIS & PV9 & 2 & 39 & A & \begin{tabular}{|l|}
$05 / 10 / 99$ \\
\end{tabular} & RB76-5418 & $50 . \mathrm{C}$ & 2,8 & 14 & 76 & 15 & 21 & 0,47 & 0,47 & 0,51 & 0,11 & 0,42 & 7,58 & $|17,37|$ & 82,92 & 58,41 \\
\hline SAO LUIS & PV9 & 2 & 39 & B & \begin{tabular}{|l|}
$05 / 10 / 99$ \\
\end{tabular} & RB76-5418 & $50 . \mathrm{C}$ & 1,3 & 6 & 68 & 54 & 43 & 0,22 & 0,25 & 0,93 & 0,58 & 30,86 & \begin{tabular}{|l|}
9,79 \\
\end{tabular} & & & \\
\hline SAO LUIS & PV9 & 2 & 40 & A & \begin{tabular}{|l|}
$05 / 10 / 99$ \\
\end{tabular} & $\begin{array}{l}\text { RB76-5418 } \\
\end{array}$ & $50 . \mathrm{C}$ & 3,2 & 8 & 68 & 38 & 21 & 0,53 & 0,31 & 0,93 & 0,97 & 0,42 & \begin{tabular}{|l|}
11,05 \\
\end{tabular} & 21,63 & 6,51 & 50,08 \\
\hline SAO LUIS & PV9 & 2 & 40 & $\bar{B}$ & \begin{tabular}{|l|}
$05 / 10 / 99$ \\
\end{tabular} & RB76-5418 & $50 . C$ & 1,6 & 5 & 71 & 64 & 43 & 0,27 & 0,21 & 0,96 & 0,78 & 0,86 & $\mid 10,58$ & & & \\
\hline SAOLUIS & PV9 & 2 & 41 & $\bar{A}$ & \begin{tabular}{|l|}
$05 / 10 / 99$ \\
\end{tabular} & RB76-5418 & $50 . \mathrm{C}$ & 1,6 & 10 & 37 & 45 & 21 & 0,27 & 0,36 & 0,19 & 1,08 & 30,42 & \begin{tabular}{|l|}
8,61 \\
\end{tabular} & 19,98 & 85,12 & 62,7 \\
\hline SAO LUIS & PV9 & 2 & 41 & $\bar{B}$ & \begin{tabular}{|l|}
$05 / 10 / 99$ \\
\end{tabular} & RB76-5418 & $50 . \mathrm{C}$ & 1,0 & 13 & 136 & 35 & 43 & 0,17 & 0,44 & 1,00 & 0,92 & 0,86 & $\mid$\begin{tabular}{|l|}
11,37 \\
\end{tabular} & & & \\
\hline SAO LUIS & PV9 & 2 & 42 & A & \begin{tabular}{|l|}
$05 / 10 / 99$ \\
\end{tabular} & RB76-5418 & $50 . \mathrm{C}$ & 1,5 & 5 & 33 & 33 & 21 & 0,25 & 0,21 & 0,17 & 0,23 & 30,42 & 5,49 & 15,00 & 80,93 & 61,36 \\
\hline SAOLUIS & PV9 & 2 & 42 & $\bar{B}$ & \begin{tabular}{|l|}
$05 / 10 / 99$ \\
\end{tabular} & RB76-5418 & $50 . \mathrm{C}$ & 0,8 & 3 & 70 & 60 & 43 & 0,13 & 0,13 & 0,95 & 0,70 & 0,86 & \begin{tabular}{|l|}
9,51 \\
\end{tabular} & & & \\
\hline SAO LUIS & PV9 & 2 & 43 & A & \begin{tabular}{|l|}
$05 / 10 / 99$ \\
\end{tabular} & $\begin{array}{l}\text { RB76-5418 } \\
\end{array}$ & $50 . \mathrm{C}$ & 3,0 & 10 & 62 & 45 & 21 & 0,50 & 0,36 & 0,87 & 1,08 & 30,42 & \begin{tabular}{|l|}
11,44 \\
\end{tabular} & 22,19 & 86,98 & $\overline{71,9}$ \\
\hline O LUIS & PVI9 & 2 & 43 & B & //99 & RB76-5418 & 50.C & 1,0 & 8 & 69 & 68 & 43 & $0,1 /$ & 0,31 & $\mid 0,94$ & 0,86 & 0,86 & \begin{tabular}{|l|}
10,75 \\
\end{tabular} & & & \\
\hline SAO LUIS & PV9 & 3 & 44 & A & \begin{tabular}{|l|}
$05 / 10 / 99$ \\
\end{tabular} & RB76-5418 & $60 . \mathrm{C}$ & 1,3 & 6 & 68 & 76 & 21 & 0,22 & 0,25 & 0,93 & 1,00 & 0,42 & \begin{tabular}{|l|}
8,83 \\
\end{tabular} & $\mid 17,31$ & 82,87 & 51,28 \\
\hline$\overline{\text { SAO LUIS }}$ & PV9 & 3 & 44 & B & 05/10/99 & RB76-5418 & $60 . \mathrm{C}$ & 0,8 & 5 & 62 & 59 & 43 & 0,13 & 0,21 & $\mid 0,87$ & 0,68 & 30,86 & \begin{tabular}{|l|}
8,48 \\
\end{tabular} & & & \\
\hline
\end{tabular}




\begin{tabular}{|c|c|c|c|c|c|c|c|c|c|c|c|c|c|c|c|c|c|c|c|c|c|}
\hline nome & & & & & & & & teores & & & & & pontuação & & & & & & & & \\
\hline Fazenda & Solo & zona & talhão & horiz & coleta & Variedade & Estágic & $\mathrm{K}$ & $\mathrm{P}$ & & 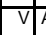 & Arg & $\mathrm{K}$ & $P$ & \begin{tabular}{|l|}
$T$ \\
\end{tabular} & \begin{tabular}{|l|l|}
$\mathrm{v}$ & \\
\end{tabular} & \begin{tabular}{|l|} 
Arg \\
\end{tabular} & NP & Ps & rod estima & $\overline{\text { od med }}$ \\
\hline AO LUIS & PV9 & 3 & 45 & $\bar{A}$ & $5 / 10 / 99$ & $\begin{array}{l}\text { RB76-5418 } \\
\end{array}$ & $60 . \mathrm{C}$ & $\overline{1,0}$ & 8 & 56 & 55 & 21 & 0,17 & 0,31 & 0,81 & 0,60 & 0,42 & 7,44 & 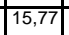 & 81,58 & 54 \\
\hline$\overline{A O L U I S}$ & PV9 & 3 & 45 & B & $5 / 10 / 99$ & RB76-5418 & $60 . \mathrm{C}$ & 0,5 & 5 & 74 & 58 & 43 & $\overline{0,08}$ & 0,21 & 0,99 & 0,66 & \begin{tabular}{|l|}
0,86 \\
\end{tabular} & 8,33 & & & \\
\hline$\overline{S A O L U I S}$ & PV9 & 3 & 46 & A & $05 / 10 / 99$ & RB76-5418 & $\begin{array}{ll}60 . c \\
\end{array}$ & 3,2 & 11 & 84 & 80 & 21 & 0,53 & 0,39 & 0,59 & 1,00 & \begin{tabular}{|l|}
0,42 \\
\end{tabular} & 9,40 & $\mid 17,37$ & 82,92 & 58,2 \\
\hline SAOLUIS & PV9 & 3 & 46 & B & 05/10/99 & RB76-5418 & \begin{tabular}{l|l}
$60 . c$ \\
\end{tabular} & 1,6 & 5 & 48 & \begin{tabular}{|l|}
74 \\
\end{tabular} & 43 & 0,27 & 0,21 & 0,24 & 0,98 & 0,86 & 7,97 & & & \\
\hline$\overline{\text { SAO LUIS }}$ & PV9 & 3 & 47 & $\bar{A}$ & $05 / 10 / 99$ & RB76-5418 & $60 . \mathrm{C}$ & 2,0 & 8 & 77 & \begin{tabular}{|l|}
63 \\
\end{tabular} & 21 & 0,33 & 0,31 & 0,52 & 0,76 & \begin{tabular}{|l|}
0,42 \\
\end{tabular} & 7,32 & 16,92 & 82,55 & 57 \\
\hline AOLUIS & PV9 & 3 & 47 & $\bar{B}$ & $5 / 10 / 99$ & RB76-5418 & $60 . \mathrm{C}$ & 1,5 & 3 & 74 & 82 & 43 & 0,25 & 0,13 & 0,99 & 1,00 & \begin{tabular}{|l|}
0,86 \\
\end{tabular} & 9,60 & & & \\
\hline$\overline{\text { SAOLUIS }}$ & PV9 & 3 & 48 & $\bar{A}$ & $05 / 10 / 99$ & $\begin{array}{l}\text { RB76-5418 } \\
\end{array}$ & $\begin{array}{ll}60 . c \\
\end{array}$ & 1,6 & 8 & 74 & 62 & 21 & 0,27 & 0,31 & 0,99 & 0,74 & 0,42 & 9,00 & 13,77 & 79,89 & 59,3 \\
\hline$\overline{\text { SAOLUIS }}$ & PV9 & 3 & 48 & $\bar{B}$ & $05 / 10 / 99$ & $\begin{array}{l}\text { RB76-5418 } \\
\end{array}$ & $60 . \mathrm{C}$ & 1,2 & 6 & 41 & 7 & 43 & 0,20 & 0,25 & 0,21 & 0,05 & 0,86 & 4,77 & & & \\
\hline SAO LUIS & PV9 & 3 & 49 & A & $05 / 10 / 99$ & $\begin{array}{l}\text { RB76-5418 } \\
\end{array}$ & $60 . \mathrm{C}$ & 1,2 & 9 & 35 & 20 & 21 & 0,20 & 0,33 & 0,18 & 0,14 & \begin{tabular}{|l|}
0,42 \\
\end{tabular} & 4,42 & $\mid 14,01$ & 80,09 & 46 \\
\hline SAOLUIS & PV9 & 3 & 49 & B & 05/10/99 & RB76-5418 & $60 . \mathrm{C}$ & 0,8 & 4 & 56 & 49 & 43 & 0,13 & 0,17 & 0,81 & 1,15 & 0,86 & 9,59 & & & \\
\hline SAO LUIS & PV9 & 3 & 50 & $\bar{A}$ & $05 / 10 / 99$ & RB76-5418 & $60 . \mathrm{C}$ & 1,3 & 11 & 58 & 27 & 21 & 0,22 & 0,39 & 0,83 & 0,19 & \begin{tabular}{|l|}
0,42 \\
\end{tabular} & \begin{tabular}{|l|}
6,82 \\
\end{tabular} & \begin{tabular}{|l|}
14,27 \\
\end{tabular} & 80,31 & 0,0 \\
\hline SAOLUIS & PV9 & 3 & 50 & $\bar{B}$ & $05 / 10 / 99$ & RB76-5418 & $\begin{array}{ll}60 . c \\
\end{array}$ & 1,6 & 3 & 49 & 36 & 43 & 0,27 & 0,13 & 0,25 & 0,93 & \begin{tabular}{|l|}
0,86 \\
\end{tabular} & 7,44 & & & \\
\hline SAOLUIS & PV9 & 3 & 52 & $\bar{A}$ & 05/10/99 & RB76-5418 & $60 . \mathrm{C}$ & 2,1 & 9 & 65 & 35 & 21 & 0,35 & 0,33 & 0,90 & 0,92 & \begin{tabular}{|l|}
0,42 \\
\end{tabular} & 9,60 & 17,92 & 83,38 & 51 \\
\hline$\overline{A O L U I S}$ & PV9 & 3 & 52 & B & $5 / 10 / 99$ & RB76-5418 & $60 . \mathrm{C}$ & $\overline{0,6}$ & 5 & 63 & 55 & 43 & 0,10 & 0,21 & 0,88 & 0,60 & 0,86 & 8,32 & & & \\
\hline$\overline{S A O L U I S}$ & PV9 & 3 & 53 & A & $05 / 10 / 99$ & $\begin{array}{l}\text { RB76-5418 } \\
\end{array}$ & $60 . \mathrm{C}$ & 2,7 & 13 & 58 & 34 & 21 & 0,45 & 0,44 & 0,83 & 0,24 & 0,42 & 7,84 & \begin{tabular}{|l|}
16,07 \\
\end{tabular} & 81,83 & 77 \\
\hline SAO LUIS & PV9 & 3 & 53 & B & 05/10/99 & RB76-5418 & $60 . \mathrm{C}$ & 1,8 & 5 & 58 & 51 & 43 & 0,30 & 0,21 & 0,83 & 0,52 & 0,86 & 8,23 & & & \\
\hline SAO LUIS & PV9 & \begin{tabular}{|l|}
8008 \\
\end{tabular} & 125 & A & $01 / 01 / 99$ & $\begin{array}{l}\text { SP78-1172 } \\
\end{array}$ & $70 . \mathrm{C}$ & 4,7 & 6 & 62 & 64 & 21 & 0,78 & 0,25 & \begin{tabular}{|l|}
0,87 \\
\end{tabular} & 0,78 & 0,42 & 8,34 & $\mid 15,33$ & 81,21 & 48 \\
\hline SAOLUIS & PV9 & \begin{tabular}{|l|}
8008 \\
\end{tabular} & 125 & $\bar{B}$ & $01 / 01 / 99$ & $\begin{array}{l}\text { SP78-1172 } \\
\end{array}$ & $70 . \mathrm{C}$ & 2,0 & 1 & 52 & 65 & 43 & 0,33 & 0,04 & \begin{tabular}{|l|}
0,77 \\
\end{tabular} & 0,80 & 0,86 & \begin{tabular}{|l|}
6,99 \\
\end{tabular} & & & \\
\hline SAO LUIS & PV9 & \begin{tabular}{|l|}
8008 \\
\end{tabular} & 128 & A & $01 / 01 / 99$ & RB72-454 & $20 . \mathrm{C}$ & 5,6 & 10 & 62 & 64 & 21 & 0,93 & 0,36 & \begin{tabular}{|l|}
0,87 \\
\end{tabular} & 0,78 & 0,42 & 15,05 & 30,78 & 94,21 & 57,2 \\
\hline SAO LUIS & PV9 & 008 & 128 & $B$ & $01 / 01 / 99$ & $\begin{array}{l}\text { RB72-454 } \\
\end{array}$ & $20 . \mathrm{C}$ & 3,3 & 5 & 74 & 48 & 43 & 0,55 & 0,21 & 0,99 & 1,13 & 0,86 & 15,73 & & & \\
\hline SAO LUIS & PV9 & \begin{tabular}{|l|}
8008 \\
\end{tabular} & 131 & A & $01 / 01 / 99$ & RB72-454 & $20 . \mathrm{C}$ & 5,6 & \begin{tabular}{c|}
6 \\
\end{tabular} & 52 & 51 & 21 & 0,93 & 0,25 & 0,77 & 0,52 & 0,42 & 13,26 & 26,15 & $\overline{90,31}$ & 62,0 \\
\hline SAO LUIS & PV9 & \begin{tabular}{|l|}
8008 \\
\end{tabular} & 131 & $B$ & $01 / 01 / 99$ & RB72-454 & $20 . \mathrm{C}$ & 3,1 & \begin{tabular}{l|l}
3 \\
\end{tabular} & 53 & 52 & 43 & 0,52 & 0,13 & 0,78 & 0,54 & 0,86 & 12,89 & & & \\
\hline SAO LUIS & PV9 & \begin{tabular}{|l|}
8008 \\
\end{tabular} & 132 & $\bar{A}$ & $01 / 01 / 99$ & RB72-454 & $20 . \mathrm{C}$ & 5,7 & 5 & 67 & 13 & 21 & 0,95 & 0,21 & 0,92 & 0,09 & 0,42 & 12,51 & 24,78 & 89,16 & 41, \\
\hline SAOLUIS & PV9 & \begin{tabular}{|l|}
8008 \\
\end{tabular} & 132 & $\bar{B}$ & $01 / 01 / 99$ & RB72-454 & $20 . \mathrm{C}$ & 3,1 & 3 & 63 & 33 & 43 & 0,52 & 0,13 & \begin{tabular}{|l|}
0,88 \\
\end{tabular} & 0,23 & 0,86 & 12,27 & & & \\
\hline SAOLUIS & PV9 & \begin{tabular}{|l|}
10010 \\
\end{tabular} & 140 & A & $01 / 01 / 99$ & RB72-454 & $20 . \mathrm{C}$ & 3,5 & 8 & 65 & 35 & 21 & 0,58 & 0,31 & 0,90 & 0,92 & 0,42 & 13,89 & 25,43 & 89,70 & 94,6 \\
\hline SAO LUIS & PV9 & \begin{tabular}{|l|}
10010 \\
\end{tabular} & 140 & $\bar{B}$ & $01 / 01 / 99$ & RB72-454 & $20 . \mathrm{C}$ & 2,1 & 3 & 72 & 27 & 43 & 0,35 & 0,13 & 0,97 & 0,19 & 0,86 & 11,54 & & & \\
\hline$\overline{\text { SAO LUIS }}$ & PV9 & \begin{tabular}{|l|}
10010 \\
\end{tabular} & 142 & $\bar{A}$ & $01 / 01 / 99$ & SP80-1842 & $20 . \mathrm{C}$ & 1,9 & 5 & 66 & 57 & 21 & 0,32 & 0,21 & 0,91 & 0,04 & \begin{tabular}{|l|}
0,42 \\
\end{tabular} & 11,62 & 22,45 & 87,2 & 42, \\
\hline SAO LUIS & PV9 & \begin{tabular}{|l|}
10010 \\
\end{tabular} & 142 & $B$ & $01 / 01 / 99$ & SP80-1842 & $20 . \mathrm{C}$ & 1,1 & \begin{tabular}{|l|l|}
3 & \\
\end{tabular} & 68 & 30 & 43 & 0,18 & 0,13 & 0,93 & 0,21 & 0,86 & 10,83 & & & \\
\hline SAO LUIS & PV9 & \begin{tabular}{|l|}
10010 \\
\end{tabular} & 145 & A & $01 / 01 / 99$ & $\begin{array}{l}\text { RB72-454 } \\
\end{array}$ & $20 . \mathrm{C}$ & 1,3 & 4 & 64 & 51 & 21 & 0,22 & 0,17 & 0,89 & 0,52 & 0,42 & 10,77 & 24,04 & 88,54 & 90,93 \\
\hline SAOLUIS & PV9 & \begin{tabular}{|l|}
10010 \\
\end{tabular} & 145 & B & $01 / 01 / 99$ & RB72-454 & $20 . \mathrm{C}$ & 0,6 & 3 & 72 & 41 & 43 & 0,10 & 0,13 & 0,97 & 1,02 & \begin{tabular}{|l|}
0,86 \\
\end{tabular} & $\begin{array}{ll}13,27 \\
\end{array}$ & & & \\
\hline SAO LUIS & PV9 & \begin{tabular}{|l|}
10010 \\
\end{tabular} & 146 & $\bar{A}$ & $01 / 01 / 99$ & RB72-454 & $20 . \mathrm{C}$ & 2,0 & 4 & 69 & 55 & 21 & 0,33 & 0,17 & 0,94 & 0,60 & 0,42 & 11,59 & 22,82 & 87,51 & 33,3 \\
\hline SAO LUIS & PV9 & 10010 & 146 & $\bar{B}$ & $01 / 01 / 99$ & RB72-454 & $20 . \mathrm{C}$ & 1,8 & 2 & 78 & 56 & 43 & 0,30 & 0,08 & 0,53 & 0,62 & 0,86 & 11,23 & & & \\
\hline \begin{tabular}{|l|} 
AREIA BRAI \\
\end{tabular} & STA B & 1 & 6 & A & 29/11/99 & $\begin{array}{l}\text { RB83-5089 } \\
\end{array}$ & $50 . \mathrm{C}$ & 0,8 & 10 & 92 & 21 & & 0,13 & 0,36 & \begin{tabular}{|l|}
0,67 \\
\end{tabular} & 0,15 & 0,00 & 5,22 & \begin{tabular}{|l|}
10,03 \\
\end{tabular} & 76,75 & 68,0 \\
\hline AREIA BRAN & STA B & 1 & 6 & B & $29 / 11 / 99$ & $\begin{array}{l}\text { RB83-5089 } \\
\end{array}$ & $50 . \mathrm{C}$ & 0,5 & 11 & 89 & 34 & & 0,08 & 0,39 & 0,64 & 0,24 & 0,00 & 4,81 & & & \\
\hline AREIA BRAN & STA B & 3 & 42 & $\bar{A}$ & $10 / 09 / 99$ & SP71-6163 & \begin{tabular}{ll|}
$70 . \mathrm{C}$ \\
\end{tabular} & $\overline{1,2}$ & 8 & 87 & 33 & & 0,20 & 0,31 & 0,62 & 0,23 & 0,00 & \begin{tabular}{|l|}
3,93 \\
\end{tabular} & 6,38 & 73,68 & $\overline{40,9}$ \\
\hline AREIA BRAN & STA B & 3 & 42 & B & $10 / 09 / 99$ & SP71-6163 & \begin{tabular}{ll|}
$70 . C$ \\
\end{tabular} & 0,8 & \begin{tabular}{|l|}
4 \\
\end{tabular} & 83 & 30 & & 0,13 & 0,17 & 0,58 & 0,21 & 0,00 & 2,45 & & & \\
\hline AREIA BRAN & STA B & 3 & 44 & A & $01 / 01 / 99$ & SP71-6163 & $80 . \mathrm{C}$ & 3,0 & 15 & 64 & 34 & & 0,50 & 0,50 & 0,89 & 0,24 & 0,00 & 5,27 & 7,27 & 74,43 & 43 \\
\hline AREIA BRAN & 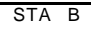 & 3 & 44 & B & \begin{tabular}{|l|}
$01 / 01 / 99$ \\
\end{tabular} & SP71-6163 & $\begin{array}{ll}80 . C \\
\end{array}$ & 1,6 & 3 & 98 & 10 & & 0,27 & 0,13 & 0,73 & 0,07 & 0,00 & 2,00 & & & \\
\hline AREIA BRAN & STA B & 6 & 58 & A & 29/07/99 & $\begin{array}{l}\text { RB72-454 } \\
\end{array}$ & $40 . \mathrm{C}$ & 0,3 & 9 & 46 & 32 & & 0,05 & 0,33 & 0,23 & 0,23 & 0,00 & 4,55 & $\mid 10,89$ & 77,47 & 81,5 \\
\hline AREIA BRAN & STA B & 6 & 58 & $\bar{B}$ & $29 / 07 / 99$ & RB72-454 & $40 . \mathrm{C}$ & 0,3 & 4 & 39 & 43 & & 0,05 & 0,17 & \begin{tabular}{|l|}
0,20 \\
\end{tabular} & 1,05 & 0,00 & 6,34 & & & \\
\hline AREIA BRAN & STA B & 6 & 65 & $\bar{A}$ & $29 / 07 / 99$ & RB76-5418 & $50 . \mathrm{C}$ & 2,1 & 19 & 61 & 22 & & 0,35 & 0,60 & 0,86 & 0,16 & 0,00 & 7,25 & $|15,65|$ & 81,47 & 46,7 \\
\hline AREIA BRAN & STA B & 6 & 65 & B & 29/07/99 & RB76-5418 & \begin{tabular}{|l|l|}
$50 . C$ \\
\end{tabular} & 1,6 & 5 & 74 & 36 & & 0,27 & 0,21 & 0,99 & 0,93 & 0,00 & 8,40 & & & \\
\hline AREIA BRAN & STA B & 6 & 66 & $\bar{A}$ & $29 / 07 / 99$ & RB76-5418 & $50 . \mathrm{C}$ & 2,1 & 19 & 78 & 33 & & 0,35 & 0,60 & 0,53 & 0,23 & 0,00 & \begin{tabular}{|l|}
6,49 \\
\end{tabular} & 12,10 & $78,4 \mathrm{C}$ & 74,2 \\
\hline AREIA BRAN & STA B & 6 & 66 & B & $29 / 07 / 99$ & RB76-5418 & $50 . \mathrm{C}$ & 1,6 & \begin{tabular}{|l|}
5 \\
\end{tabular} & 51 & 33 & & 0,27 & 0,21 & 0,76 & 0,23 & 0,00 & 5,61 & & & \\
\hline AREIA BRAN & STA B & 6 & 67 & A & 29/07/99 & $\begin{array}{l}\text { RB83-5089 } \\
\end{array}$ & \begin{tabular}{ll|}
$40 . \mathrm{C}$ & \\
\end{tabular} & 0,6 & \begin{tabular}{l|}
6 \\
\end{tabular} & 74 & 13 & & 0,10 & 0,25 & 0,99 & 0,09 & 0,00 & 6,47 & $\mid 12,21$ & 78,58 & 99,0 \\
\hline \begin{tabular}{|l|} 
AREIA BRAN \\
\end{tabular} & STA B & 6 & 67 & B & 29/07/99 & $\begin{array}{l}\text { RB83-5089 } \\
\end{array}$ & $40 . \mathrm{C}$ & 0,1 & 3 & 73 & 20 & & 0,02 & 0,13 & 0,98 & 0,14 & 0,00 & 5,74 & & & \\
\hline AREIA BRAN & STA B & 6 & 68 & $\bar{A}$ & $29 / 07 / 99$ & RB83-5089 & \begin{tabular}{ll|}
$40 . \mathrm{C}$ & \\
\end{tabular} & 0,6 & 3 & 55 & 30 & & 0,10 & 0,13 & 0,80 & 0,21 & 0,00 & 5,81 & 12,73 & $\overline{79,0}$ & $\overline{71,8}$ \\
\hline AREIA BRAN & STA B & 6 & 68 & $\bar{B}$ & $29 / 07 / 99$ & RB83-5089 & $40 . \mathrm{C}$ & 0,1 & \begin{tabular}{|l|}
3 \\
\end{tabular} & 84 & 80 & & $\overline{0,02}$ & 0,13 & 0,59 & 1,00 & 0,00 & $6,9,92$ & & & \\
\hline AREIA BRAN & STA B & 6 & 69 & A & 29/07/99 & RB83-5089 & $40 . \mathrm{C}$ & 0,6 & \begin{tabular}{|l|}
6 \\
\end{tabular} & 70 & 17 & & 0,10 & 0,25 & 0,95 & 0,12 & 0,00 & 6,44 & 11,23 & 77,7 & 61,3 \\
\hline IA BRAN & STA B & 6 & 69 & B & 107/99 & RB83-5089 & $40 . \mathrm{C}$ & 0, & \begin{tabular}{|l|}
3 \\
\end{tabular} & 87 & 26 & &, 02 & 0,13 & 0,62 & 0,18 & 0,00 & 4,79 & & & \\
\hline AREIA BRAN & $\begin{array}{l}\text { STA B } \\
\end{array}$ & 6 & 74 & A & 29/07/99 & RB83-5089 & $30 . \mathrm{C}$ & 1,0 & \begin{tabular}{|l|}
8 \\
\end{tabular} & 75 & 30 & & 0,17 & 0,31 & 0,50 & 0,21 & 0,00 & 6,41 & 14,67 & 80,65 & 115,8 \\
\hline AREIA BRAN & STA B & 6 & 74 & $\mathrm{~B}$ & 29/07/99 & $\begin{array}{l}\text { RB83-5089 } \\
\end{array}$ & $30 . \mathrm{C}$ & 0,5 & \begin{tabular}{|l|}
3 \\
\end{tabular} & 92 & 76 & & 0,08 & 0,13 & 0,67 & 1,00 & 0,00 & 8,26 & & & \\
\hline \begin{tabular}{|l|} 
AREIA BRAN \\
\end{tabular} & $\begin{array}{l}\text { STA B } \\
\end{array}$ & 6 & 75 & $\bar{A}$ & $29 / 07 / 99$ & RB80-6043 & $30 . \mathrm{C}$ & \begin{tabular}{|l|}
0,5 \\
\end{tabular} & \begin{tabular}{|l|}
11 \\
\end{tabular} & 94 & 55 & & 0,08 & 0,39 & 0,69 & 0,60 & 0,00 & 8,59 & $\mid 15,84$ & 81,63 & 103,7 \\
\hline AREIA BRAN & STA B & 6 & 75 & B & 29/07/99 & $\begin{array}{l}\text { RB80-6043 } \\
\end{array}$ & $30 . \mathrm{C}$ & 0,3 & \begin{tabular}{|l|}
1 \\
\end{tabular} & 54 & 53 & & 0,05 & 0,04 & 0,79 & 0,56 & 0,00 & 7,25 & & & \\
\hline AREIA BRAN & STA B & 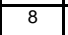 & 94 & A & $01 / 01 / 99$ & RB72-454 & $20 . C$ & 1,8 & \begin{tabular}{|l|}
7 \\
\end{tabular} & 82 & 62 & & 0,30 & 0,28 & $0,57 \mid$ & 0,74 & 0,00 & 10,09 & 18,93 & 84,23 & 55,6 \\
\hline AREIA BRAN & STA B & 8 & 94 & B & $01 / 01 / 99$ & RB72-454 & $20 . \mathrm{C}$ & \begin{tabular}{|l|}
0,8 \\
\end{tabular} & \begin{tabular}{|l|}
2 \\
\end{tabular} & 52 & 58 & & 0,13 & 0,08 & 0,77 & 0,66 & 0,00 & 8,84 & & & \\
\hline EEIA BRAN & STA B & 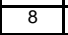 & 101 & $\mathrm{~A}$ & $01 / 01 / 99$ & RB72-454 & $20 . \mathrm{C}$ & 0,9 & \begin{tabular}{|l|}
14 \\
\end{tabular} & 335 & 38 & & 0,15 & 0,47 & \begin{tabular}{|l|}
1,00 \\
\end{tabular} & 0,97 & 0,00 & 11,60 & 22,9 & 7,6 & 74 \\
\hline EIA BRAN & STA B & 8 & 101 & B & $01 / 01 / 99$ & RB72-454 & $20 . \mathrm{C}$ & 0,9 & 21 & 218 & 66 & & 0,15 & 0,65 & 1,00 & 0,82 & 0,00 & 11,39 & & & \\
\hline SEIA BRAN & STA B & 8 & 102 & $\bar{A}$ & 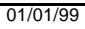 & RB72-454 & $20 . \mathrm{C}$ & 1,2 & \begin{tabular}{|l|}
6 \\
\end{tabular} & 70 & 68 & & 0,20 & 0,25 & 0,95 & 0,86 & 0,00 & 10,83 & 18,71 & 84,05 & 51,2 \\
\hline AREIA BRAN & STA B & 8 & 102 & $\bar{B}$ & $01 / 01 / 99$ & RB72-454 & $20 . \mathrm{C}$ & 0,7 & \begin{tabular}{|l|}
3 \\
\end{tabular} & 37 & 40 & & 0,12 & 0,13 & 0,19 & 1,00 & 0,00 & 7,88 & & & \\
\hline AREIA BRAN & STA B & 5 & \begin{tabular}{ll|}
111 \\
\end{tabular} & $A$ & 11/11/99 & RB72-454 & $50 . \mathrm{C}$ & \begin{tabular}{|l|}
0,3 \\
\end{tabular} & \begin{tabular}{|l|}
3 \\
\end{tabular} & 62 & 24 & & 0,05 & 0,13 & 0,87 & 0,17 & 0,00 & 4,17 & 10,09 & 76,80 & 52,2 \\
\hline AREIA BRAN & STA B & 5 & 1111 & B & $11 / 11 / 99$ & RB72-454 & $50 . \mathrm{C}$ & $\begin{array}{l}0,1 \\
\end{array}$ & \begin{tabular}{|l|}
1 \\
\end{tabular} & 66 & \begin{tabular}{|c|}
69 \\
\end{tabular} & & 0,02 & 0,04 & 0,91 & 0,88 & 0,00 & 5,92 & & & \\
\hline REIA BRAN & STA B & 5 & 112 & $\bar{A}$ & $11 / 11 / 99$ & RB72-454 & $50 . \mathrm{C}$ & 0,5 & \begin{tabular}{|l|}
4 \\
\end{tabular} & 87 & 26 & & 0,08 & 0,17 & 0,62 & 0,18 & 0,00 & 4,29 & 7,60 & 74,71 & 78,2 \\
\hline EIA BRAN & STA B & 5 & 112 & B & $11 / 11 / 99$ & RB72-454 & $50 . \mathrm{C}$ & 0,3 & 1 & 75 & 30 & & 0,05 & {$[0,04$} & 0,50 & 0,21 & 0,00 & 3,31 & & & \\
\hline EIABRAN & STA B & 5 & 114 & A & $11 / 11 / 99$ & RB72-454 & $50 . \mathrm{C}$ & 0,5 & \begin{tabular}{|l|l|}
6 & \\
\end{tabular} & 25 & & & 0,08 & 0,25 & 1,00 & 0,56 & 0,0 & 6,81 & 13,90 & 80,00 & 42,9 \\
\hline REIA BRAN & STA B & 5 & 114 & B & $11 / 11 / 99$ & RB72-454 & $50 . C$ & 0,3 & \begin{tabular}{|l|}
5 \\
\end{tabular} & 155 & 69 & & 0,05 & 0,21 & 1,00 & 0,88 & 0,00 & 7,09 & & & \\
\hline AREIA BRAN & STA B & 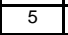 & 116 & A & 11/11/99 & RB72-454 & $50 . \mathrm{C}$ & \begin{tabular}{|l|}
0,5 \\
\end{tabular} & 6 & 30 & & & 0,08 & 0,25 & 0,83 & 0,19 & 0,0 & 5,19 & $\overline{0,97}$ & 7,54 & 00,0 \\
\hline
\end{tabular}




\begin{tabular}{|c|c|c|c|c|c|c|c|c|c|c|c|c|c|c|c|c|c|c|c|c|c|}
\hline nome & & & & & & & & teores & & & & & pontuação & & & & & & & & \\
\hline Fazenda & Solo & zona & talhão & horiz & coleta & Variedade & Estágic & $\mathrm{K}$ & ${ }^{P}$ & & $\mathrm{~V} / \mathrm{A}$ & Arg & $\mathrm{K}$ & $P$ & \begin{tabular}{|l|}
$T$ \\
\end{tabular} & V & Arg & $N P$ & Ps & od estima & od medida \\
\hline$\overline{\text { REIA BRAN }}$ & $\begin{array}{l}\text { STA } B \\
\end{array}$ & 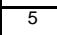 & 116 & $\bar{B}$ & /111/99 & RB72-454 & $50 . \mathrm{C}$ & $\overline{0,3}$ & 8 & 76 & 63 & & 0,05 & 0,31 & 0,51 & 0,76 & 0,00 & \begin{tabular}{|l|}
5,78 \\
\end{tabular} & & & \\
\hline REIA BRAN & STA B & 3 & 42a & A & $1 / 01 / 99$ & SP71-6163 & $80 . \mathrm{C}$ & 2,4 & 8 & 122 & 72 & & $\overline{0,40}$ & 0,31 & \begin{tabular}{|l|}
0,97 \\
\end{tabular} & $\mid 0,94$ & 0,00 & \begin{tabular}{|l|}
6,72 \\
\end{tabular} & 8,61 & 75,55 & 40,96 \\
\hline$\overline{\text { AREIA BRAN }}$ & $\begin{array}{l}\text { STA } B \\
\end{array}$ & 3 & $42 a$ & 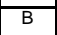 & \begin{tabular}{|l|}
$01 / 01 / 99$ \\
\end{tabular} & SP71-6163 & $80 . \mathrm{C}$ & 1,1 & 4 & 84 & 30 & & 0,18 & 0,17 & 0,59 & 0,21 & 0,00 & \begin{tabular}{|l|}
1,88 \\
\end{tabular} & & & \\
\hline REIA BRAN & STA B & 6 & $65 a$ & A & 06/07/99 & RB76-5418 & \begin{tabular}{l|l}
$60 . c$ \\
\end{tabular} & 2,1 & 19 & 41 & 39 & & 0,35 & 0,60 & 0,21 & 0,98 & \begin{tabular}{|l|}
0,00 \\
\end{tabular} & \begin{tabular}{|l|}
6,79 \\
\end{tabular} & 14,85 & 80,80 & $\overline{46}$ \\
\hline REIA BRAN & STA B & 6 & $65 a$ & $\bar{B}$ & $06 / 07 / 99$ & RB76-5418 & $60 . \mathrm{C}$ & 1,6 & 5 & 156 & 48 & & 0,27 & 0,21 & 1,00 & 1,13 & 0,00 & 8,05 & & & \\
\hline REIA BRAN & STA B & 6 & $66 a$ & $\bar{A}$ & $6 / 07 / 99$ & RB76-5418 & $60 . \mathrm{C}$ & 2,1 & 19 & 88 & 52 & & 0,35 & 0,60 & 0,63 & 0,54 & 0,00 & \begin{tabular}{|l|}
6,74 \\
\end{tabular} & 13,62 & 79,77 & 74,2 \\
\hline AREIA BRAN & $\begin{array}{l}\text { STA B } \\
\text { ST }\end{array}$ & 6 & $66 a$ & $\bar{B}$ & $\overline{06 / 07 / 99}$ & $\begin{array}{l}\text { RB76-5418 } \\
\end{array}$ & $\begin{array}{ll}60 . c \\
\end{array}$ & 1,6 & 5 & 51 & 39 & & 0,27 & 0,21 & 0,76 & 0,98 & 0,00 & \begin{tabular}{|l|}
6,88 \\
\end{tabular} & & & \\
\hline$\overline{\text { AREIA BRAN }}$ & STA B & 6 & $67 a$ & A & $\overline{06 / 07 / 99}$ & $\begin{array}{l}\text { RB83-5089 } \\
\end{array}$ & $50 . \mathrm{C}$ & 0,6 & 6 & 52 & $\mid 19$ & & 0,10 & 0,25 & 0,77 & 0,13 & 0,00 & \begin{tabular}{|l|}
4,97 \\
\end{tabular} & \begin{tabular}{|l|}
9,71 \\
\end{tabular} & 76,48 & 99 \\
\hline AREIA BRAN & STA B & 6 & $67 a$ & 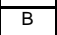 & $06 / 07 / 99$ & $\begin{array}{l}\text { RB83-5089 } \\
\end{array}$ & $50 . \mathrm{C}$ & 0,1 & 3 & 63 & 33 & & 0,02 & 0,13 & 0,88 & 0,23 & $\mid 0,00$ & \begin{tabular}{|l|}
4,74 \\
\end{tabular} & & & \\
\hline AREIA BRAN & STA B & 6 & $68 \mathrm{a}$ & A & 06/07/99 & RB83-5089 & $50 . \mathrm{C}$ & 0,1 & 3 & 74 & 29 & & 0,02 & 0,13 & 0,99 & 0,21 & 0,00 & 4 & 11,91 & 78,33 & 71, \\
\hline AREIA BRAN & STA B & 6 & $68 \mathrm{a}$ & B & $\overline{06 / 07 / 99}$ & RB83-5089 & $50 . \mathrm{C}$ & 0,1 & 3 & 74 & 75 & & $\overline{0,02}$ & 0,13 & 0,99 & 1,00 & 0 & \begin{tabular}{|l|}
7,15 \\
\end{tabular} & & & \\
\hline \begin{tabular}{|l} 
AREIA BRAN \\
\end{tabular} & STA B & 6 & $69 a$ & A & $06 / 07 / 99$ & RB83-5089 & 50.c & 0,6 & 6 & 69 & 63 & & 0,10 & 0,25 & 0,94 & 0,76 & \begin{tabular}{|l|}
0,00 \\
\end{tabular} & 7,35 & 10,93 & 77,51 & 61,3 \\
\hline AREIA BRAN & STA B & 6 & $69 \mathrm{a}$ & $\bar{B}$ & $06 / 07 / 99$ & RB83-5089 & $50 . \mathrm{C}$ & 0,1 & 3 & 90 & 11 & & 0,02 & 0,13 & 0,65 & 0,08 & 0,00 & \begin{tabular}{|l|}
3,58 \\
\end{tabular} & & & \\
\hline$\overline{\text { AREIA BRAN }}$ & $\begin{array}{l}\text { STA B } \\
\text { ST }\end{array}$ & 6 & $74 a$ & $\bar{A}$ & $3 / 07 / 99$ & $\begin{array}{l}\text { RB83-5089 } \\
\end{array}$ & $40 . \mathrm{C}$ & 1,0 & 8 & 89 & $\mid 19$ & & 0,17 & 0,31 & 0,64 & 0,13 & 0,00 & \begin{tabular}{|l|}
5,62 \\
\end{tabular} & \begin{tabular}{|l|}
9,69 \\
\end{tabular} & 76,46 & 115,8 \\
\hline AREIA BRAN & STA B & 6 & $74 a$ & $B$ & 08/07/99 & $\begin{array}{l}\text { RB83-5089 } \\
\end{array}$ & $40 . \mathrm{C}$ & 0,5 & 3 & 75 & 14 & & 0,08 & 0,13 & 0,50 & 0,10 & 0,00 & \begin{tabular}{|l|}
4,07 \\
\end{tabular} & & & \\
\hline AREIA BRAN & STA B & 6 & $75 a$ & A & $06 / 07 / 99$ & $\begin{array}{l}\text { RB80-6043 } \\
\end{array}$ & $40 . \mathrm{C}$ & 0,5 & \begin{tabular}{|l|}
11 \\
\end{tabular} & 106 & 84 & & 0,08 & 0,39 & 0,81 & 1,00 & 0,00 & \begin{tabular}{|l|}
9,17 \\
\end{tabular} & 14,10 & 80,17 & 103,7 \\
\hline AREIA BRAN & STA B & 6 & $75 a$ & B & $06 / 07 / 99$ & $\begin{array}{l}\text { RB80-6043 } \\
\end{array}$ & $40 . \mathrm{C}$ & 0,3 & 1 & 40 & 60 & & 0,05 & 0,04 & 0,20 & 0,70 & 0,00 & \begin{tabular}{|l|}
4,93 \\
\end{tabular} & & & \\
\hline SAO PEDRO & STA B & 1 & 4 & $\bar{A}$ & $01 / 01 / 99$ & RB72-454 & $70 . \mathrm{C}$ & 1,5 & 6 & 108 & 83 & & 0,25 & 0,25 & 0,83 & 1,00 & 0,00 & \begin{tabular}{|l|}
6,62 \\
\end{tabular} & $\mid 11,19$ & 77,72 & 71,7 \\
\hline SAO PEDRO & STA B & 1 & 4 & B & $01 / 01 / 99$ & RB72-454 & $70 . \mathrm{C}$ & 1,3 & 5 & 92 & 54 & & 0,22 & 0,21 & \begin{tabular}{|l|}
0,67 \\
\end{tabular} & 0,58 & 0,00 & \begin{tabular}{|l|}
4,58 \\
\end{tabular} & & & \\
\hline SAO PEDRO & STA B & 1 & 6 & A & 29/11/99 & RB72-454 & $70 . \mathrm{C}$ & 7,8 & 61 & 80 & 52 & & 1,00 & 1,00 & 0,55 & 0,54 & 0,00 & \begin{tabular}{|l|}
9,05 \\
\end{tabular} & $\mid 17,42$ & 82,96 & 72,6 \\
\hline SAO PEDRO & STA B & 1 & 6 & B & 29/11/99 & RB72-454 & $70 . \mathrm{C}$ & 3,0 & \begin{tabular}{|l|}
43 \\
\end{tabular} & 109 & 65 & & 0,50 & 1,00 & 0,84 & 0,80 & 0,00 & \begin{tabular}{|l|}
8,37 \\
\end{tabular} & & & \\
\hline SAO PEDRO & STA B & 6 & 12 & A & $20 / 10 / 99$ & $\begin{array}{l}\text { SP70-1143 } \\
\end{array}$ & $80 . \mathrm{C}$ & 0,6 & 8 & 79 & 56 & & 0,10 & 0,31 & 0,54 & 0,62 & 0,00 & \begin{tabular}{|l|}
2,75 \\
\end{tabular} & 4,78 & 72,33 & 48,0 \\
\hline SAO PEDRO & STA B & 6 & 12 & B & $20 / 10 / 99$ & SP70-1143 & $80 . \mathrm{C}$ & 0,3 & 1 & 78 & 64 & & 0,05 & 0,04 & 0,53 & 0,78 & 0,00 & \begin{tabular}{|l|}
2,03 \\
\end{tabular} & & & \\
\hline SAO PEDRO & STA B & 3 & 32 & A & $01 / 01 / 99$ & RB72-454 & $20 . \mathrm{C}$ & 2,2 & \begin{tabular}{|l|}
20 \\
\end{tabular} & 50 & 60 & & 0,37 & 0,63 & 0,75 & 0,70 & 0,00 & 12,58 & 22,74 & 87,44 & 83,2 \\
\hline SAO PEDRO & STA B & 3 & 32 & $\bar{B}$ & $01 / 01 / 99$ & RB72-454 & $20 . \mathrm{C}$ & 0,8 & 8 & 50 & 60 & & 0,13 & 0,31 & 0,75 & 0,70 & 0,00 & $\mid$\begin{tabular}{|l|}
10,17 \\
\end{tabular} & & & \\
\hline SAO PEDRO & STA B & 3 & 33 & A & $01 / 01 / 99$ & RB72-454 & $20 . \mathrm{C}$ & 3,7 & 37 & 50 & 60 & & 0,62 & 1,00 & 0,75 & 0,70 & 0,00 & \begin{tabular}{|l|}
14,45 \\
\end{tabular} & 23,85 & 88,38 & 132,6 \\
\hline$\overline{S A O P E D R O}$ & STA B & 3 & 33 & B & $01 / 01 / 99$ & RB72-454 & $20 . \mathrm{C}$ & 1,6 & 8 & 52 & 34 & & 0,27 & 0,31 & \begin{tabular}{|l|}
0,77 \\
\end{tabular} & 0,24 & 0,00 & \begin{tabular}{|l|}
9,40 \\
\end{tabular} & & & \\
\hline SAO PEDRO & STA B & 3 & 34 & A & $01 / 01 / 99$ & RB72-454 & $20 . \mathrm{C}$ & 2,1 & \begin{tabular}{|l|}
26 \\
\end{tabular} & 52 & 34 & & 0,35 & 0,78 & 0,77 & 0,24 & 0,00 & \begin{tabular}{|l|}
11,06 \\
\end{tabular} & 20,32 & 85,40 & 123,8 \\
\hline SAO PEDRO & STA B & 3 & 34 & $B$ & $01 / 01 / 99$ & RB72-454 & $20 . \mathrm{C}$ & 0,9 & 5 & 42 & 47 & & 0,15 & 0,21 & 0,21 & 1,12 & 0,00 & \begin{tabular}{|l|}
9,26 \\
\end{tabular} & & & \\
\hline SAO PEDRO & STA B & 5 & 36 & A & $01 / 01 / 99$ & RB72-454 & $20 . \mathrm{C}$ & 2,8 & \begin{tabular}{|l|}
41 \\
\end{tabular} & 44 & 49 & & 0,47 & 1,00 & 0,22 & 1,15 & 0,00 & 13,16 & 27,20 & 91,19 & 98,6 \\
\hline SAO PEDRO & STA B & 5 & 36 & B & $01 / 01 / 99$ & RB72-454 & $20 . \mathrm{C}$ & 2,0 & \begin{tabular}{|l|}
83 \\
\end{tabular} & 56 & 73 & & 0,33 & 1,00 & 0,81 & 0,96 & 0,00 & $\mid 14,04$ & & & \\
\hline SAO PEDRO & STA B & 5 & 37 & A & $01 / 01 / 99$ & \begin{tabular}{ll|} 
RB72-454 \\
\end{tabular} & $20 . \mathrm{C}$ & 4,2 & \begin{tabular}{|l|}
10 \\
\end{tabular} & 42 & 61 & & 0,70 & 0,36 & 0,21 & 0,72 & 0,00 & $\mid$\begin{tabular}{|l|}
10,47 \\
\end{tabular} & 21,33 & 86,2 & 93,71 \\
\hline SAO PEDRO & STA B & 5 & 37 & $B$ & 01/01/99 & RB72-454 & $20 . \mathrm{C}$ & 4,5 & 11 & 42 & 61 & & 0,75 & 0,39 & 0,21 & 0,72 & 0,00 & $\mid 10,86$ & & & \\
\hline \begin{tabular}{|l|} 
SAO PEDRO \\
\end{tabular} & STA B & 5 & 38 & A & $01 / 01 / 99$ & RB72-454 & $20 . \mathrm{C}$ & 11,9 & \begin{tabular}{|l|}
57 \\
\end{tabular} & 42 & 61 & & 1,00 & 1,00 & 0,21 & 0,72 & 0,00 & \begin{tabular}{|l|l|}
13,37 \\
\end{tabular} & 23,80 & 88,33 & 48,0 \\
\hline SAO PEDRO & STA B & 5 & 38 & $\bar{B}$ & $01 / 01 / 99$ & RB72-454 & $20 . \mathrm{C}$ & 6,2 & \begin{tabular}{|l|}
20 \\
\end{tabular} & 47 & 27 & & 1,00 & 0,63 & 0,24 & 0,19 & 0,00 & $\mid 10,43$ & & & \\
\hline SAO PEDRO & STA B & 8 & 49 & A & $01 / 01 / 99$ & RB72-454 & $20 . \mathrm{C}$ & 1,5 & \begin{tabular}{|l|}
7 \\
\end{tabular} & 47 & 27 & & 0,25 & 0,28 & 0,24 & 0,19 & 0,00 & 7,06 & 16,02 & $81,7 \mathrm{~s}$ & 96,5 \\
\hline SAO PEDRO & STA B & 8 & 49 & $\bar{B}$ & $01 / 01 / 99$ & RB72-454 & $20 . \mathrm{C}$ & 0,6 & \begin{tabular}{|l|}
7 \\
\end{tabular} & 48 & 41 & & 0,10 & 0,28 & \begin{tabular}{|l|}
0,24 \\
\end{tabular} & 1,02 & 0,00 & \begin{tabular}{|l|}
8,96 \\
\end{tabular} & & & \\
\hline SAO PEDRO & STA B & 8 & 52 & $\bar{A}$ & $01 / 01 / 99$ & RB72-454 & $20 . \mathrm{C}$ & 1,1 & 32 & 43 & 62 & & $\overline{0,18}$ & 0,93 & 0,22 & 0,74 & 0,00 & $\mid 10,77$ & 22,21 & $\overline{86,95}$ & 78,0 \\
\hline SAO PEDRO & STA B & 8 & 52 & B & $01 / 01 / 99$ & RB72-454 & $20 . \mathrm{C}$ & 0,8 & \begin{tabular}{|l|}
16 \\
\end{tabular} & 50 & 74 & & 0,13 & 0,53 & 0,75 & 0,98 & 0,00 & $|11,44|$ & & & \\
\hline SAO PEDRO & STA B & 8 & 53 & $\bar{A}$ & $01 / 01 / 99$ & RB72-454 & $20 . \mathrm{C}$ & 4,0 & \begin{tabular}{|l|}
19 \\
\end{tabular} & 93 & 31 & & 0,67 & 0,60 & 0,68 & 0,22 & 0,00 & 11,00 & 24,16 & $\overline{88,6}$ & $\overline{09,4 \mathrm{C}}$ \\
\hline SAO PEDRO & STA B & 8 & 53 & $\bar{B}$ & 99 & RB72-454 & $20 . \mathrm{C}$ & 3,6 & \begin{tabular}{|l|}
18 \\
\end{tabular} & 112 & 35 & & 0,60 & 0,58 & 0,87 & 0,92 & 0,00 & $\mid 13,16$ & & & \\
\hline SAO PEDRO & STA B & 8 & 55 & A & $01 / 01 / 99$ & RB72-454 & $20 . \mathrm{C}$ & 1,2 & \begin{tabular}{|l|}
24 \\
\end{tabular} & 112 & 35 & & 0,20 & 0,73 & \begin{tabular}{|l|}
0,87 \\
\end{tabular} & 0,92 & 0,00 & 12,71 & 22,29 & 87,06 & 107,8 \\
\hline SAO PEDRO & STA B & 8 & 55 & B & $01 / 01 / 99$ & RB72-454 & $20 . \mathrm{C}$ & 0,4 & \begin{tabular}{|l|}
24 \\
\end{tabular} & 93 & 31 & & 0,07 & 0,73 & 0,68 & 0,22 & 0,00 & \begin{tabular}{|l|}
9,58 \\
\end{tabular} & & & \\
\hline SAO PEDRO & STA B & 8 & 58 & $\bar{A}$ & $01 / 01 / 99$ & RB72-454 & $20 . \mathrm{C}$ & 3,2 & \begin{tabular}{|l|}
11 \\
\end{tabular} & 85 & 38 & & 0,53 & 0,39 & 0,60 & 0,97 & 0,00 & 12,35 & 23,69 & 8,2 & 117,36 \\
\hline SAO PEDRO & STA B & 8 & 58 & $\bar{B}$ & $01 / 01 / 99$ & RB72-454 & $20 . \mathrm{C}$ & 1,4 & \begin{tabular}{|l|l|}
6 & \\
\end{tabular} & 114 & 36 & & 0,23 & 0,25 & 0,89 & 0,93 & 0,00 & $\mid 11,35$ & & & \\
\hline SAO PEDRO & STA B & 7 & 64 & A & 01/01/99 & RB72-454 & $20 . \mathrm{C}$ & 7,1 & \begin{tabular}{|l|}
80 \\
\end{tabular} & 114 & 36 & & 1,00 & 1,00 & 0,89 & 0,93 & 0,00 & $\mid 16,12$ & 28,39 & 92,20 & 72,15 \\
\hline SAO PEDRO & STA B & 7 & 64 & $\bar{B}$ & $01 / 01 / 99$ & RB72-454 & $20 . \mathrm{C}$ & 3,9 & \begin{tabular}{|l|}
14 \\
\end{tabular} & 85 & 38 & & 0,65 & 0,47 & 0,60 & 0,97 & 0,00 & \begin{tabular}{|l|}
12,27 \\
\end{tabular} & & & \\
\hline SAO PEDRO & STA B & 7 & 66 & $\bar{A}$ & $01 / 01 / 99$ & RB72-454 & $20 . \mathrm{C}$ & 0,8 & \begin{tabular}{|l|}
44 \\
\end{tabular} & 40 & 45 & & 0,13 & 1,00 & 0,20 & 1,08 & 0,00 & \begin{tabular}{|l|}
11,83 \\
\end{tabular} & 23,49 & 88,0 & 90,19 \\
\hline SAO PEDRO & STA B & 7 & 66 & $B$ & 01/01/99 & RB72-454 & $20 . \mathrm{C}$ & 0,5 & \begin{tabular}{|l|}
47 \\
\end{tabular} & 39 & 48 & & 0,08 & 1,00 & 0,20 & 1,13 & 0,00 & $\mid 11,66$ & & & \\
\hline $\mathrm{DPE}$ & STA B & 7 & 68 & A & 99 & 772-454 & $20 . \mathrm{C}$ & 2,2 & \begin{tabular}{|l|}
4 \\
\end{tabular} & 52 & 40 & & (1 & 0,17 & \begin{tabular}{|l|}
0,77 \\
\end{tabular} & 1,00 & 0,00 & 10,89 & 21,70 &, 56 & 86,0 \\
\hline SAO PEDRO & STA B & 7 & 68 & B & $01 / 01 / 99$ & RB72-454 & $20 . C$ & 2,3 & \begin{tabular}{|l|}
3 \\
\end{tabular} & 51 & 45 & & 0,38 & 0,13 & 0,76 & 1,08 & 0,00 & $\mid 10,81$ & & & \\
\hline SAO PEDRO & STA B & 0 & $13 a$ & $\mathrm{~A}$ & $01 / 01 / 99$ & SP71-6163 & $80 . \mathrm{C}$ & 1,7 & \begin{tabular}{|l|l}
5 \\
\end{tabular} & 113 & 43 & & 0,28 & 0,21 & 0,88 & 1,05 & 0,00 & \begin{tabular}{|l|}
4,87 \\
\end{tabular} & 7,11 & 74,26 & 40,75 \\
\hline SAO PEDRO & STA B & 6 & 13a & B & $01 / 01 / 99$ & SP71-6163 & $80 . \mathrm{C}$ & 2,0 & \begin{tabular}{|l|}
3 \\
\end{tabular} & 80 & 52 & & 0,33 & 0,13 & 0,55 & 0,54 & 0,00 & \begin{tabular}{|l|}
2,25 \\
\end{tabular} & & & \\
\hline B.VISTA SH & & 3 & 43 & $\mathrm{~A}$ & $01 / 01 / 99$ & $\begin{array}{l}\text { RB85-5113 } \\
\end{array}$ & $20 . \mathrm{C}$ & 1,4 & \begin{tabular}{|l|l|}
6 & \\
\end{tabular} & 96 & 84 & & 0,23 & 0,25 & \begin{tabular}{|l|}
0,71 \\
\end{tabular} & 1,00 & 0,00 & \begin{tabular}{|l|}
10,78 \\
\end{tabular} & 19,39 & 84,63 & 52,14 \\
\hline ISTA SH & & 3 & 43 & B & \begin{tabular}{|l|}
$01 / 01 / 99$ \\
\end{tabular} & RB85-5113 & $20 . \mathrm{C}$ & 1,1 & \begin{tabular}{|l|}
2 \\
\end{tabular} & 39 & 48 & & 0,18 & 0,08 & 0,20 & 1,13 & {$[0,00$} & \begin{tabular}{|l|}
8,61 \\
\end{tabular} & & & \\
\hline B.VISTA SH & & 3 & 48 & $\bar{A}$ & $01 / 01 / 99$ & $\begin{array}{l}\text { RB83-5054 } \\
\end{array}$ & $20 . \mathrm{C}$ & 3,4 & \begin{tabular}{|l|}
6 \\
\end{tabular} & 32 & 21 & & 0,57 & 0,25 & \begin{tabular}{|l|}
0,16 \\
\end{tabular} & 0,15 & 0,00 & 7,80 & 17,12 & 82,71 & 148,1 \\
\hline B.VISTA SH & & 3 & 48 & $\mathrm{~B}$ & $01 / 01 / 99$ & RB83-5054 & $20 . \mathrm{C}$ & 3,2 & \begin{tabular}{|l|}
3 \\
\end{tabular} & 29 & 37 & & 0,53 & 0,13 & 0,15 & 0,95 & 0,00 & \begin{tabular}{|l|}
9,31 \\
\end{tabular} & & & \\
\hline VISTA SH & & 3 & 53 & A & $01 / 01 / 99$ & RB85-5113 & $20 . \mathrm{C}$ & 1,4 & \begin{tabular}{|l|}
9 \\
\end{tabular} & 53 & 58 & & 0,23 & 0,33 & 0,78 & 0,66 & 0,00 & 10,30 & 19,21 & 84,47 & 64,6 \\
\hline VISTA SH & & 3 & 53 & $\bar{B}$ & $01 / 01 / 99$ & $\begin{array}{l}\text { RB85-5113 } \\
\end{array}$ & $20 . \mathrm{C}$ & 1,5 & \begin{tabular}{|l|}
3 \\
\end{tabular} & 36 & 44 & & 0,25 & 0,13 & 0,18 & \begin{tabular}{|l|}
1,07 \\
\end{tabular} & 0,00 & \begin{tabular}{|l|}
8,92 \\
\end{tabular} & & & \\
\hline B.VISTA SH & & 3 & 54 & $\bar{A}$ & $01 / 01 / 99$ & RB85-5113 & $20 . \mathrm{C}$ & 1,3 & \begin{tabular}{|l|}
4 \\
\end{tabular} & 177 & 93 & & 0,22 & 0,17 & \begin{tabular}{|l|}
1,00 \\
\end{tabular} & 1,00 & 0,00 & 11,50 & 19,91 & 85,06 & $52,9 \varsigma$ \\
\hline B.VISTA SH & & 3 & 54 & B & $01 / 01 / 99$ & RB85-5113 & $20 . C$ & \begin{tabular}{|l|}
0,8 \\
\end{tabular} & \begin{tabular}{|l|}
3 \\
\end{tabular} & 39 & 43 & & 0,13 & 0,13 & \begin{tabular}{|l|}
0,20 \\
\end{tabular} & 1,05 & 0,00 & \begin{tabular}{|l|}
8,41 \\
\end{tabular} & & & \\
\hline B.VISTA SH & & 3 & 56 & A & $01 / 01 / 99$ & $\begin{array}{l}\text { RB85-5113 } \\
\end{array}$ & $20 . \mathrm{C}$ & \begin{tabular}{|l|}
1,7 \\
\end{tabular} & \begin{tabular}{|l|}
5 \\
\end{tabular} & 111 & 69 & & 0,28 & 0,21 & 0,86 & 0,88 & 0,00 & 11,20 & 21,36 & 86,28 & 98,64 \\
\hline \begin{tabular}{|l} 
B.VISTA SH \\
\end{tabular} & & 3 & 56 & B & $01 / 01 / 99$ & RB85-5113 & $20 . \mathrm{C}$ & 2,3 & \begin{tabular}{|c|}
3 \\
\end{tabular} & 67 & 58 & & 0,38 & 0,13 & 0,92 & 0,66 & 0,00 & $\mid$\begin{tabular}{|l|}
10,17 \\
\end{tabular} & & & \\
\hline VISTA SH & & 6 & 94 & $\bar{A}$ & $01 / 01 / 99$ & RB76-5418 & $50 . \mathrm{C}$ & 2,1 & \begin{tabular}{|l|}
9 \\
\end{tabular} & 88 & 61 & & 0,35 & 0,33 & \begin{tabular}{|l|}
0,63 \\
\end{tabular} & 0,72 & 0,00 & \begin{tabular}{|l|}
7,38 \\
\end{tabular} & 13,64 & 79,79 & $\overline{73,3}$ \\
\hline VISTA SH & & 6 & 94 & B & $01 / 01 / 99$ & RB76-5418 & $50 . \mathrm{C}$ & 1,0 & \begin{tabular}{|l|}
4 \\
\end{tabular} & 118 & & & 0, & 0,17 & 0,93 & 0,50 & 0,00 & \begin{tabular}{|l|}
6,27 \\
\end{tabular} & & & \\
\hline 3.VISTA SH & & 6 & 97 & A & $01 / 01 / 99$ & RB76-5418 & $50 . C$ & 4,4 & \begin{tabular}{|l|}
12 \\
\end{tabular} & 110 & 52 & & 0,73 & 0,42 & 0,85 & 0,54 & 0,00 & \begin{tabular}{|l|}
8,82 \\
\end{tabular} & 15,20 & 81,10 & 73,3 \\
\hline B.VISTA SH & & 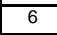 & 97 & B & $01 / 01 / 99$ & RB76-5418 & $50 . \mathrm{C}$ & \begin{tabular}{|l|}
1,0 \\
\end{tabular} & \begin{tabular}{|l|}
3 \\
\end{tabular} & $\cdots$ & & & 0,17 & 0,13 & {$[, 00]$} & 0 & {$[0,00$} & 6,38 & & & \\
\hline
\end{tabular}




\begin{tabular}{|c|c|c|c|c|c|c|c|c|c|c|c|c|c|c|c|c|c|c|c|c|c|}
\hline nome & & & & & & & & teores & & & & & pontuação & & & & & & & & \\
\hline Fazenda & Solo & zona & talhão & horiz & coleta & Variedade & Estágio & $\mathrm{K}$ & $P$ & & vt & Arg & $\mathrm{K}$ & $P$ & $T$ & \begin{tabular}{|l|l}
$\mathrm{V}$ & $\mathrm{g}$ \\
\end{tabular} & \begin{tabular}{|l|} 
Arg \\
\end{tabular} & $\mathrm{NP}$ & Ps & prod estima & prod medida \\
\hline B.VISTA SH & & 7 & 164 & $\bar{A}$ & \begin{tabular}{|l|}
$01 / 01 / 99$ \\
\end{tabular} & RB76-5418 & 50.C & 4,7 & 6 & 35 & 60 & & 0,78 & 0,25 & 0,18 & 0,70 & 0,00 & \begin{tabular}{|l|}
6,78 \\
\end{tabular} & $|10,99|$ & 77,56 & 62 \\
\hline B.VISTA SH & & 7 & 164 & B & \begin{tabular}{|l|}
$01 / 01 / 99$ \\
\end{tabular} & $\begin{array}{l}\text { RB76-5418 } \\
\end{array}$ & $50 . \mathrm{C}$ & 4,9 & 3 & 28 & 21 & & 0,82 & 0,13 & 0,14 & 0,15 & 0,00 & \begin{tabular}{|l|}
4,22 \\
\end{tabular} & & & \\
\hline BOA ESPERA & & 19 & 3 & $\bar{A}$ & \begin{tabular}{|l|}
$04 / 08 / 99$ \\
\end{tabular} & RB82-5336 & $40 . \mathrm{C}$ & 0,3 & 9 & 66 & 21 & & 0,05 & 0,33 & 0,91 & 0,15 & \begin{tabular}{|l|}
0,00 \\
\end{tabular} & 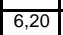 & 12,04 & 78,44 & 42,94 \\
\hline BOA ESPERA & & 19 & 3 & B & \begin{tabular}{|l|}
$04 / 08 / 99$ \\
\end{tabular} & RB82-5336 & $40 . \mathrm{C}$ & 1,0 & 4 & 106 & 32 & & 0,17 & \begin{tabular}{|l|}
0,17 \\
\end{tabular} & 0,81 & 0,23 & \begin{tabular}{|l|}
0,00 \\
\end{tabular} & 5,84 & & & \\
\hline BOA ESPERA & & 19 & 4 & $\bar{A}$ & \begin{tabular}{|l|}
$04 / 08 / 99$ \\
\end{tabular} & RB82-5336 & $40 . \mathrm{C}$ & 0,5 & 18 & 105 & 23 & & 0,08 & 0,58 & 0,80 & 0,16 & 0,00 & 6,89 & 15,50 & 81,35 & 42,59 \\
\hline BOA ESPERA & & 19 & 4 & $\bar{B}$ & \begin{tabular}{|l|}
$04 / 08 / 99$ \\
\end{tabular} & RB82-5336 & $40 . \mathrm{C}$ & 0,5 & 31 & 157 & 30 & & 0,08 & 0,90 & 1,00 & $\overline{0,21}$ & 0,00 & \begin{tabular}{|l|}
8,61 \\
\end{tabular} & & & \\
\hline BRASIL III & & 46 & 5 & $\bar{A}$ & \begin{tabular}{|l|}
$29 / 11 / 99$ \\
\end{tabular} & RB72-454 & $50 . \mathrm{C}$ & $\overline{0,6}$ & 5 & 37 & 32 & & 0,10 & 0,21 & 0,19 & 0,23 & \begin{tabular}{|l|}
0,00 \\
\end{tabular} & 3,29 & \begin{tabular}{|l|}
7,75 \\
\end{tabular} & 74,83 & 72,9 \\
\hline BRASIL III & & 46 & 5 & B & \begin{tabular}{|l|}
$29 / 11 / 99$ \\
\end{tabular} & RB72-454 & $50 . \mathrm{C}$ & 0,5 & \begin{tabular}{|l|l|}
3 & \\
\end{tabular} & 53 & 28 & & 0,08 & 0,13 & 0,78 & 0,20 & \begin{tabular}{|l|}
0,00 \\
\end{tabular} & 4,46 & & & \\
\hline MANDACARU & & 74 & 26 & A & \begin{tabular}{|l|}
$29 / 11 / 99$ \\
\end{tabular} & RB72-454 & $50 . \mathrm{C}$ & 0,3 & 4 & 154 & 21 & & 0,05 & 0,17 & 1,00 & 0,15 & 0 & \begin{tabular}{|l|}
4,62 \\
\end{tabular} & 10,60 & 77,23 & 62,60 \\
\hline MANDACARU & & 74 & 26 & B & \begin{tabular}{|l|}
$29 / 11 / 99$ \\
\end{tabular} & RB72-454 & $50 . \mathrm{C}$ & 0,3 & 4 & 106 & 67 & & 0,05 & \begin{tabular}{|l|}
0,17 \\
\end{tabular} & 0,81 & 0,84 & \begin{tabular}{|l|}
0,00 \\
\end{tabular} & \begin{tabular}{|l|}
5,98 \\
\end{tabular} & & & \\
\hline MANDACARU & & 74 & 27 & A & \begin{tabular}{|l|}
$29 / 11 / 99$ \\
\end{tabular} & RB72-454 & $50 . \mathrm{C}$ & 0,3 & 5 & 94 & 67 & & 0,05 & 0,21 & 0,69 & 0,84 & 0,00 & 6,04 & 12,66 & 78,96 & 49,57 \\
\hline MANDACARU & & 74 & 27 & B & \begin{tabular}{|l|}
$29 / 11 / 99$ \\
\end{tabular} & RB72-454 & $50 . \mathrm{C}$ & 0,3 & 3 & 113 & 75 & & 0,05 & 0,13 & 0,88 & 1,00 & 0 & \begin{tabular}{|l|}
6,62 \\
\end{tabular} & & & \\
\hline \begin{tabular}{|l|} 
MANDACARU \\
\end{tabular} & & 74 & 29 & A & \begin{tabular}{|l|}
$29 / 11 / 99$ \\
\end{tabular} & RB72-454 & $50 . \mathrm{C}$ & 0,3 & 6 & 116 & 49 & & 0,05 & 0,25 & 0,91 & 1,15 & 0,00 & 7,53 & $\mid 13,34$ & 79,53 & 85,55 \\
\hline MANDACARU & & 74 & 29 & $\bar{B}$ & \begin{tabular}{|l|}
$29 / 11 / 99$ \\
\end{tabular} & RB72-454 & $50 . \mathrm{C}$ & 0,3 & 8 & 105 & 59 & & 0,05 & $\mid 0,31$ & 0,80 & 0,68 & \begin{tabular}{|l|}
0,00 \\
\end{tabular} & \begin{tabular}{|l|}
5,81 \\
\end{tabular} & & & \\
\hline MANDACARU & & 74 & 34 & $\bar{A}$ & \begin{tabular}{|l|}
$29 / 11 / 99$ \\
\end{tabular} & RB72-454 & $50 . \mathrm{C}$ & 0,3 & 4 & 51 & $\mid 17$ & & 0,05 & 0,17 & 0,76 & 0,12 & \begin{tabular}{|l|}
0,00 \\
\end{tabular} & \begin{tabular}{|l|}
3,97 \\
\end{tabular} & $|10,62|$ & 77,24 & 87,72 \\
\hline MANDACARU & & 74 & 34 & $B$ & \begin{tabular}{|l|}
$29 / 11 / 99$ \\
\end{tabular} & RB72-454 & $50 . \mathrm{C}$ & 0,3 & 3 & 110 & 41 & & 0,05 & 0,13 & 0,85 & 1,02 & \begin{tabular}{|l|}
0,00 \\
\end{tabular} & 6,65 & & & \\
\hline \begin{tabular}{|l|} 
MANDACARU \\
\end{tabular} & & 75 & 37 & A & \begin{tabular}{|l|}
$29 / 11 / 99$ \\
\end{tabular} & RB72-454 & $50 . \mathrm{C}$ & 0,3 & \begin{tabular}{l|}
6 \\
\end{tabular} & 127 & 66 & & 0,05 & 0,25 & 1,00 & 0,82 & 0,00 & \begin{tabular}{|l|}
6,89 \\
\end{tabular} & 13,19 & 79,40 & 55 \\
\hline MANDACARU & & 75 & 37 & B & \begin{tabular}{|l|}
$29 / 11 / 99$ \\
\end{tabular} & RB72-454 & $50 . \mathrm{C}$ & 0,3 & 3 & 116 & 67 & & 0,05 & 0,13 & 0,91 & 0,84 & 0,00 & 6,3, & & & \\
\hline MANDACARU & & 75 & 39 & A & \begin{tabular}{|l|}
$29 / 11 / 99$ \\
\end{tabular} & RB72-454 & $50 . \mathrm{C}$ & 0,6 & 6 & 45 & 37 & & 0,10 & 0,25 & 0,23 & 0,95 & 0,00 & 5,10 & \begin{tabular}{|l|}
9,07 \\
\end{tabular} & 75,94 & 46,6 \\
\hline MANDACARU & & 75 & 39 & B & \begin{tabular}{|l|}
$29 / 11 / 99$ \\
\end{tabular} & RB72-454 & $50 . \mathrm{C}$ & 0,5 & 6 & 36 & 58 & & 0,08 & \begin{tabular}{|c|}
0,25 \\
\end{tabular} & 0,18 & 0,66 & 0,00 & \begin{tabular}{|l|}
3,97 \\
\end{tabular} & & & \\
\hline MANDACARU & & 75 & 49 & $\bar{A}$ & \begin{tabular}{|l|}
$29 / 11 / 99$ \\
\end{tabular} & RB72-454 & $50 . \mathrm{C}$ & 0,5 & 4 & 65 & 80 & & 0,08 & 0,17 & 0,90 & 1,00 & 0,00 & 7,20 & $\mid 14,12$ & 80,19 & 47, \\
\hline MANDACARU & & 75 & 49 & $B$ & \begin{tabular}{|l|}
$29 / 11 / 99$ \\
\end{tabular} & RB72-454 & $50 . \mathrm{C}$ & 0,6 & 4 & 64 & 78 & & 0,10 & $|0,17|$ & 0,89 & 1,00 & 0,00 & \begin{tabular}{|l|}
6,92 \\
\end{tabular} & & & \\
\hline MONTE BELO & & 2 & 13 & A & \begin{tabular}{|l|}
$11 / 11 / 99$ \\
\end{tabular} & RB72-454 & $60 . \mathrm{C}$ & 0,6 & 6 & 56 & 67 & & 0,10 & \begin{tabular}{|l|}
0,25 \\
\end{tabular} & 0,81 & \begin{tabular}{l|l}
0,84 \\
\end{tabular} & 0,00 & 5,93 & 11,29 & 77,81 & 141,80 \\
\hline MONTE BELO & & 2 & 13 & B & \begin{tabular}{|l|}
$11 / 11 / 99$ \\
\end{tabular} & RB72-454 & $60 . \mathrm{C}$ & 0,0 & 1 & 97 & \begin{tabular}{|l|}
46 \\
\end{tabular} & & 0,00 & 0,04 & 0,72 & 1,10 & 0,00 & 5,36 & & & \\
\hline MONTE BELO & & 2 & 15 & A & \begin{tabular}{|l|}
$11 / 11 / 99$ \\
\end{tabular} & RB80-6043 & $60 . \mathrm{C}$ & 0,3 & 8 & 71 & 69 & & 0,05 & 0,31 & 0,96 & 0,88 & 0,00 & \begin{tabular}{|l|}
6,51 \\
\end{tabular} & 12,06 & 78,46 & 49,7 \\
\hline MONTE BELO & & 2 & 15 & B & \begin{tabular}{|l|}
$11 / 11 / 99$ \\
\end{tabular} & RB80-6043 & $60 . \mathrm{C}$ & 0,3 & 3 & 95 & 76 & & 0,05 & 0,13 & 0,70 & 1,00 & 0,00 & 5,55 & & & \\
\hline MONTE BELO & & 2 & 18 & $\bar{A}$ & \begin{tabular}{|l|}
$11 / 11 / 99$ \\
\end{tabular} & RB72-454 & 60.C & 0,3 & 5 & 118 & 73 & & 0,05 & 0,21 & 0,93 & 0,96 & 0,00 & 6,22 & \begin{tabular}{|l|}
9,80 \\
\end{tabular} & 76,55 & 84 \\
\hline MONTE BELO & & 2 & 18 & B & \begin{tabular}{|l|}
$11 / 11 / 99$ \\
\end{tabular} & RB72-454 & $60 . \mathrm{C}$ & 0,6 & 4 & 37 & 67 & & 0,10 & $|0,17|$ & 0,19 & 0,84 & 0,00 & 3,58 & & & \\
\hline MONTE BELO & & 2 & 24 & A & \begin{tabular}{|l|}
$11 / 11 / 99$ \\
\end{tabular} & RB80-6043 & $60 . \mathrm{C}$ & 0,5 & \begin{tabular}{|c|}
1 \\
\end{tabular} & 152 & \begin{tabular}{|l|}
42 \\
\end{tabular} & & 0,08 & 0,04 & 1,00 & 1,03 & 0,00 & \begin{tabular}{|l|}
6,55 \\
\end{tabular} & 10,25 & 76,94 & 60,9 \\
\hline MONTE BELO & & 2 & 24 & B & \begin{tabular}{|l|}
$11 / 11 / 99$ \\
\end{tabular} & RB80-6043 & 60.C & 0,3 & 1 & 137 & 20 & & 0,05 & 0,04 & 1,00 & 0,14 & 0,00 & 3,70 & & & \\
\hline MORRO ALTO & & 1 & 1 & A & \begin{tabular}{|l|}
$01 / 01 / 99$ \\
\end{tabular} & RB72-454 & $20 . \mathrm{C}$ & 0,7 & 3 & 179 & 32 & & 0,12 & 0,13 & 1,00 & 0,23 & 0,00 & 8,68 & $\mid 16,53$ & 82,22 & 55,0 \\
\hline MORRO ALTO & & 1 & 1 & B & \begin{tabular}{|l|}
$01 / 01 / 99$ \\
\end{tabular} & RB72-454 & $20 . \mathrm{C}$ & 0,8 & \begin{tabular}{|l|}
2 \\
\end{tabular} & 175 & 14 & & 0,13 & 0,08 & 1,00 & 0,10 & 0,00 & 7,85 & & & \\
\hline PARAISO & & 4 & 20 & A & \begin{tabular}{|l|}
$30 / 12 / 99$ \\
\end{tabular} & SP79-1011 & $70 . \mathrm{C}$ & 3,3 & 11 & 65 & 47 & & 0,55 & 0,39 & 0,90 & 1,12 & 0,00 & \begin{tabular}{|l|}
9,02 \\
\end{tabular} & 10,29 & 76,96 & 67 \\
\hline PARAISO & & 4 & 20 & B & \begin{tabular}{|l|}
$30 / 12 / 99$ \\
\end{tabular} & SP79-1011 & $70 . \mathrm{C}$ & 1,2 & 4 & 34 & 26 & & 0,20 & 0,17 & 0,17 & 0,18 & 0,00 & 1,26 & & & \\
\hline PARAISO & & 5 & 27 & A & \begin{tabular}{|l|}
$01 / 01 / 99$ \\
\end{tabular} & RB72-454 & $20 . \mathrm{C}$ & 2,9 & \begin{tabular}{|l|}
30 \\
\end{tabular} & 67 & 29 & & 0,48 & 0,88 & 0,92 & 0,21 & 0,00 & 11,58 & 22,66 & 87,37 & 87,15 \\
\hline PARAISO & & 5 & 27 & B & \begin{tabular}{|l|}
$01 / 01 / 99$ \\
\end{tabular} & RB72-454 & $20 . \mathrm{C}$ & 2,3 & 4 & 110 & 41 & & 0,38 & 0,17 & 0,85 & 1,02 & 0,00 & $\mid 11,08$ & & & \\
\hline PARAISO & & 5 & 29 & $\bar{A}$ & \begin{tabular}{|l|}
$01 / 01 / 99$ \\
\end{tabular} & RB72-454 & $20 . \mathrm{C}$ & 3,6 & 15 & 92 & 48 & & 0,60 & 0,50 & $\mid 0,67$ & 1,13 & 0,00 & 12,91 & 22,15 & 86,94 & 66,95 \\
\hline PARAISO & & 5 & 29 & $\mathrm{~B}$ & \begin{tabular}{|l|}
$01 / 01 / 99$ \\
\end{tabular} & RB72-454 & $20 . \mathrm{C}$ & 1,4 & \begin{tabular}{|c|}
8 \\
\end{tabular} & 46 & 39 & & 0,23 & 0,31 & 0,23 & 0,98 & 0,00 & 9,24 & & & \\
\hline PARAISO & & 6 & 36 & A & \begin{tabular}{|l|}
$10 / 09 / 99$ \\
\end{tabular} & RB76-5418 & $80 . \mathrm{C}$ & 1,0 & \begin{tabular}{|l|}
16 \\
\end{tabular} & 41 & 24 & & 0,17 & \begin{tabular}{|l|}
0,53 \\
\end{tabular} & 0,21 & 0,17 & 0,00 & \begin{tabular}{|l|}
1,78 \\
\end{tabular} & \begin{tabular}{|l|}
2,01 \\
\end{tabular} & 70,00 & 60,94 \\
\hline PARAISO & & 6 & 36 & B & \begin{tabular}{|l|}
$10 / 09 / 99$ \\
\end{tabular} & RB76-5418 & $80 . \mathrm{C}$ & $\overline{0,6}$ & 5 & 42 & 33 & & 0,10 & 0,21 & 0,21 & 0,23 & 0,00 & \begin{tabular}{|l|}
0,23 \\
\end{tabular} & & & \\
\hline PARAISO & & 6 & 38 & A & \begin{tabular}{|l|}
$01 / 01 / 99$ \\
\end{tabular} & RB72-454 & $20 . \mathrm{C}$ & 3,9 & \begin{tabular}{|l|}
18 \\
\end{tabular} & 75 & 22 & & 0,65 & 0,58 & 0,50 & 0,16 & 0,00 & \begin{tabular}{|l|}
9,69 \\
\end{tabular} & 16,72 & 82,38 & 87,9 \\
\hline PARAISO & & 6 & 38 & B & \begin{tabular}{|l|}
$01 / 01 / 99$ \\
\end{tabular} & RB72-454 & $20 . \mathrm{C}$ & 3,5 & 4 & 26 & 23 & & 0,58 & 0,17 & 0,13 & 0,16 & 0,00 & 7,03 & & & \\
\hline PARAISO & & 6 & 40 & $\bar{A}$ & \begin{tabular}{|l|}
$01 / 01 / 99$ \\
\end{tabular} & RB72-454 & $20 . \mathrm{C}$ & 2,9 & 6 & 55 & 54 & & 0,48 & 0,25 & 0,80 & 0,58 & 0,00 & 10,62 & 18,30 & 83,71 & 97,52 \\
\hline PARAISO & & 6 & 40 & B & \begin{tabular}{|l|}
$01 / 01 / 99$ \\
\end{tabular} & RB72-454 & $20 . \mathrm{C}$ & 1,7 & 3 & 87 & 33 & & 0,28 & 0,13 & \begin{tabular}{|l|}
0,62 \\
\end{tabular} & 0,23 & 0,00 & 7,69 & & & \\
\hline PARAISO & & 6 & 42 & A & \begin{tabular}{|l|}
$30 / 12 / 99$ \\
\end{tabular} & RB72-454 & $80 . \mathrm{C}$ & 1,0 & 10 & 110 & $10 \mid$ & & 0,17 & 0,36 & 0,85 & 0,07 & 0,00 & \begin{tabular}{|l|}
3,22 \\
\end{tabular} & \begin{tabular}{|l|}
5,84 \\
\end{tabular} & 73,22 & 69,65 \\
\hline PARAISO & & 6 & 42 & B & 30/12/99 & $\begin{array}{l}\text { RB72-454 } \\
\end{array}$ & $80 . \mathrm{C}$ & 0,5 & 5 & 70 & \begin{tabular}{|l|}
8 \\
\end{tabular} & & 0,08 & 0,21 & 0,95 & 0,06 & 0,00 & \begin{tabular}{|l|}
2,62 \\
\end{tabular} & & & \\
\hline PARAISO & & 6 & 45 & A & \begin{tabular}{|l|}
$01 / 01 / 99$ \\
\end{tabular} & SP79-1011 & $50 . \mathrm{C}$ & 0,8 & \begin{tabular}{l|l}
6 \\
\end{tabular} & 63 & 46 & & 0,13 & \begin{tabular}{|l|}
0,25 \\
\end{tabular} & 0,88 & 1,10 & 0,00 & 8,59 & 14,12 & 80,18 & 51,5 \\
\hline PARAISO & & 6 & 45 & B & \begin{tabular}{|l|}
$01 / 01 / 99$ \\
\end{tabular} & SP79-1011 & $50 . \mathrm{C}$ & $\overline{0,5}$ & 14 & 56 & \begin{tabular}{|l|}
7 \\
\end{tabular} & & 0,08 & $\mid 0,47$ & 0,81 & 0,05 & 0,00 & 5,52 & & & \\
\hline PARAISO & & 6 & 46 & A & \begin{tabular}{|l|}
$30 / 12 / 99$ \\
\end{tabular} & SP79-1011 & $50 . \mathrm{C}$ & 1,0 & \begin{tabular}{|l|}
10 \\
\end{tabular} & 50 & 24 & & 0,17 & 0,36 & 0,75 & 0,17 & 0,00 & \begin{tabular}{|l|}
6,07 \\
\end{tabular} & $\mid 10,80$ & 77,40 & 62,66 \\
\hline PARAISO & & 6 & 46 & $B$ & \begin{tabular}{|l|}
$30 / 12 / 99$ \\
\end{tabular} & SP79-1011 & $50 . \mathrm{C}$ & 0,5 & 5 & 93 & 22 & & 0,08 & $|0,21|$ & 0,68 & 0,16 & 0,00 & \begin{tabular}{|l|}
4,73 \\
\end{tabular} & & & \\
\hline PARAISO & & 8 & 62 & A & \begin{tabular}{|l|}
$10 / 09 / 99$ \\
\end{tabular} & RB76-5418 & $90 . \mathrm{C}$ & 7,2 & \begin{tabular}{|l|}
5 \\
\end{tabular} & 99 & $27 \mid$ & & 1,00 & 0,21 & 0,74 & 0,19 & 0,00 & \begin{tabular}{|l|}
4,17 \\
\end{tabular} & \begin{tabular}{|l|}
7,56 \\
\end{tabular} & 74,67 & 61,38 \\
\hline PARAISO & & 8 & 62 & B & \begin{tabular}{|l|}
$10 / 09 / 99$ \\
\end{tabular} & RB76-5418 & $90 . \mathrm{C}$ & 4,2 & \begin{tabular}{|l|}
3 \\
\end{tabular} & 82 & 58 & & 0,70 & 0,13 & 0,57 & 0,66 & 0,00 & 3,39 & & & \\
\hline PARAISO & & 8 & 63 & A & \begin{tabular}{|l|}
$10 / 09 / 99$ \\
\end{tabular} & RB76-5418 & $90 . \mathrm{C}$ & 4,5 & 5 & 114 & \begin{tabular}{|l|l|}
4 \\
\end{tabular} & & 0,75 & 0,21 & 0,89 & 0,03 & 0,00 & \begin{tabular}{|l|}
3,31 \\
\end{tabular} & 4,72 & 72,28 & 58,24 \\
\hline PARAISO & & 8 & 63 & B & \begin{tabular}{|l|}
$10 / 09 / 99$ \\
\end{tabular} & $\begin{array}{l}\text { RB76-5418 } \\
\end{array}$ & $90 . \mathrm{C}$ & 2,5 & 3 & 78 & \begin{tabular}{|l|}
7 \\
\end{tabular} & & 0,42 & 0,13 & 0,53 & 0,05 & 0,00 & \begin{tabular}{|l|}
1,41 \\
\end{tabular} & & & \\
\hline PORT FELIZ & & 1 & 5 & A & \begin{tabular}{|l|}
$01 / 01 / 99$ \\
\end{tabular} & RB72-454 & $20 . \mathrm{C}$ & 2,3 & \begin{tabular}{|l|}
30 \\
\end{tabular} & 92 & 21 & & 0,38 & $\mid 0,88$ & 0,67 & 0,15 & 0,00 & 10,51 & $\mid 20,17$ & 85,27 & 113,95 \\
\hline PORT FELIZ & & 1 & 5 & B & \begin{tabular}{|l|}
$01 / 01 / 99$ \\
\end{tabular} & RB72-454 & $20 . \mathrm{C}$ & 0,7 & \begin{tabular}{|l|l|}
5 & \\
\end{tabular} & 86 & 39 & & 0,12 & 0,21 & 0,61 & 0,98 & 0,00 & $9, \mid 66$ & & & \\
\hline \begin{tabular}{|l|} 
R.TANQUINH \\
\end{tabular} & & 54 & 2 & $\bar{A}$ & |01/01/99 & SP79-2312 & $40 . \mathrm{C}$ & 2,5 & 5 & 92 & \begin{tabular}{|l|}
43 \\
\end{tabular} & & $\overline{0,42}$ & 0,21 & \begin{tabular}{|l|}
0,67 \\
\end{tabular} & 1,05 & 0,00 & \begin{tabular}{|l|}
10,86 \\
\end{tabular} & 22,06 & 86,87 & 87,90 \\
\hline \begin{tabular}{|l|} 
R.TANQUINH \\
\end{tabular} & & 54 & 2 & B & \begin{tabular}{|l|}
$01 / 01 / 99$ \\
\end{tabular} & SP79-2312 & $40 . \mathrm{C}$ & 1,3 & 8 & 139 & 36 & & 0,22 & $\mid 0,31$ & 1,00 & 0,93 & 0,00 & 11,19 & & & \\
\hline S.LOURENCO & & 10 & 6 & A & \begin{tabular}{|l|}
$30 / 12 / 99$ \\
\end{tabular} & RB78-5148 & $50 . \mathrm{C}$ & 0,5 & 5 & 39 & 20 & & 0,08 & 0,21 & 0,20 & 0,14 & 0,00 & \begin{tabular}{|l|}
2,79 \\
\end{tabular} & \begin{tabular}{|l|}
8,02 \\
\end{tabular} & 75,06 & 53,99 \\
\hline S.LOURENCO & & 10 & 6 & B & \begin{tabular}{|l|}
$30 / 12 / 99$ \\
\end{tabular} & RB78-5148 & $50 . \mathrm{C}$ & $\overline{0,3}$ & 11 & 75 & 54 & & 0,05 & 0,39 & 0,50 & 0,58 & 0,00 & 5,23 & & & \\
\hline S.LOURENCO & & 11 & 19 & A & \begin{tabular}{|l|}
$29 / 11 / 99$ \\
\end{tabular} & RB78-5148 & $50 . \mathrm{C}$ & 1,6 & \begin{tabular}{|l|}
5 \\
\end{tabular} & 41 & 56 & & 0,27 & 0,21 & 0,21 & 0,62 & 0,00 & 4,58 & \begin{tabular}{|l|}
9,26 \\
\end{tabular} & 76,10 & 52,16 \\
\hline S.LOURENCO & & 11 & 19 & B & \begin{tabular}{|l|}
$29 / 11 / 99$ \\
\end{tabular} & RB78-5148 & $50 . \mathrm{C}$ & 0,8 & \begin{tabular}{|l|l|}
5 \\
\end{tabular} & 43 & 69 & & 0,13 & 0,21 & 0,22 & 0,88 & 0,00 & 4,69 & & & \\
\hline S.LOURENCO & & 11 & 22 & $\bar{A}$ & \begin{tabular}{|l|}
$29 / 11 / 99$ \\
\end{tabular} & RB78-5148 & $50 . \mathrm{C}$ & 0,6 & 11 & 360 & \begin{tabular}{|l|}
29 \\
\end{tabular} & & 0,10 & 0,39 & 1,00 & 0,21 & 0,00 & \begin{tabular}{|l|}
5,54 \\
\end{tabular} & 11,16 & 77,70 & 42,51 \\
\hline S.LOURENCO & & 11 & 22 & B & \begin{tabular}{|l|}
$29 / 11 / 99$ \\
\end{tabular} & RB78-5148 & $50 . \mathrm{C}$ & 0,6 & 16 & 191 & 21 & & 0,10 & 0,53 & 1,00 & 0,15 & 0,00 & 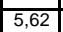 & & & \\
\hline STA.JUL.II & & 42 & 30 & $\bar{A}$ & \begin{tabular}{|l|}
$01 / 01 / 99$ \\
\end{tabular} & SP70-1143 & $\begin{array}{ll}60 . \mathrm{C} \\
\end{array}$ & 1,2 & 5 & 42 & 47 & & 0,20 & 0,21 & 0,21 & 1,12 & 0,00 & 4,76 & 7,51 & 74,63 & 61,34 \\
\hline STA.JUL.II & & 42 & 30 & B & \begin{tabular}{|l|}
$01 / 01 / 99$ \\
\end{tabular} & SP70-1143 & 60.C & 1,3 & \begin{tabular}{|l|}
3 \\
\end{tabular} & 42 & 52 & & $\overline{0,22}$ & 0,13 & 0,21 & 0,54 & 0,00 & \begin{tabular}{|l|}
2,75 \\
\end{tabular} & & & \\
\hline STA.JULIA & & 15 & 7 & $\bar{A}$ & \begin{tabular}{|l|}
$30 / 12 / 99$ \\
\end{tabular} & RB78-5148 & $60 . \mathrm{C}$ & 1,6 & 5 & 36 & 30 & & 0,27 & 0,21 & 0,18 & 0,21 & 0,00 & 2,90 & \begin{tabular}{|l|}
4,60 \\
\end{tabular} & 72,18 & $\overline{46,2}$ \\
\hline
\end{tabular}




\section{9}

\begin{tabular}{|c|c|c|c|c|c|c|c|c|c|c|c|c|c|c|c|c|c|c|c|c|c|}
\hline nome & & & & & & & & teores & & & & & pontuação & & & & & & & & \\
\hline Fazenda & Solo & \begin{tabular}{|l} 
zona \\
\end{tabular} & talhão & horiz & coleta & Variedade & Estágio & $\bar{K}$ & $\bar{P}$ & & $\mathrm{v}$ & Arg & $\mathrm{K}$ & $P$ & T & $\bar{v}$ & \begin{tabular}{|l|l|} 
Arg \\
\end{tabular} & NP & \begin{tabular}{|l|} 
Ps \\
\end{tabular} & prod estima & prod medida \\
\hline \begin{tabular}{|l|} 
STA.JULIA \\
\end{tabular} & & 15 & 7 & B & $30 / 12 / 99$ & RB78-5148 & $60 . C$ & 0,6 & 3 & 46 & 26 & & 0,10 & 0,13 & 0,23 & 0,18 & 0,00 & \begin{tabular}{|l|}
1,69 \\
\end{tabular} & & & \\
\hline \begin{tabular}{|l|} 
STA.JULIA \\
\end{tabular} & & 15 & 9 & A & $30 / 12 / 99$ & RB78-5148 & $60 . C$ & 2,3 & 29 & 28 & 14 & & 0,38 & 0,85 & 0,14 & 0,10 & 0,00 & \begin{tabular}{|l|}
5,09 \\
\end{tabular} & \begin{tabular}{|l|l|}
8,58 \\
\end{tabular} & 75,52 & 40,67 \\
\hline \begin{tabular}{|l|} 
STA.JULIA \\
\end{tabular} & & 15 & 9 & B & $30 / 12 / 99$ & RB78-5148 & $60 . C$ & 1,0 & 23 & 34 & 14 & & 0,17 & 0,70 & 0,17 & 0,10 & 0,00 & \begin{tabular}{|l|}
3,48 \\
\end{tabular} & & & \\
\hline \begin{tabular}{|l|} 
STA.JULIA \\
\end{tabular} & & 15 & 12 & $\bar{A}$ & \begin{tabular}{|l|}
$30 / 12 / 99$ \\
\end{tabular} & RB78-5148 & $60 . C$ & \begin{tabular}{|l|}
3,2 \\
\end{tabular} & 6 & 37 & 35 & & 0,53 & 0,25 & 0,19 & 0,92 & 0,00 & \begin{tabular}{|l|}
5,88 \\
\end{tabular} & 12,58 & 78,89 & 45,10 \\
\hline STA.JULIA & & 15 & $\overline{12}$ & $\bar{B}$ & $30 / 12 / 99$ & RB78-5148 & $60 . C$ & 1,0 & 4 & 73 & 47 & & 0,17 & 0,17 & 0,98 & 1,12 & 0,00 & 6,69 & & & \\
\hline TRIPOLI & & 56 & 4 & A & \begin{tabular}{|l|}
$06 / 10 / 99$ \\
\end{tabular} & RB78-5148 & $60 . C$ & \begin{tabular}{|l|}
0,3 \\
\end{tabular} & 5 & 89 & 47 & & 0,05 & 0,21 & 0,64 & 1,12 & 0,00 & \begin{tabular}{|l|}
6,27 \\
\end{tabular} & \begin{tabular}{|l|l}
11,26 \\
\end{tabular} & 77,78 & 52,88 \\
\hline \begin{tabular}{|l|} 
TRIPOLI \\
\end{tabular} & & 56 & 4 & $\bar{B}$ & \begin{tabular}{|l|}
$06 / 10 / 99$ \\
\end{tabular} & $\begin{array}{l}\text { RB78-5148 } \\
\end{array}$ & $60 . C$ & \begin{tabular}{|l|}
0,1 \\
\end{tabular} & 1 & 92 & 36 & & 0,02 & 0,04 & 0,67 & 0,93 & 0,00 & \begin{tabular}{|l|}
4,99 \\
\end{tabular} & & & \\
\hline TRIPOLI & & 56 & 5 & $\bar{A}$ & 06/10/99 & $\begin{array}{l}\text { RB78-5148 } \\
\end{array}$ & $60 . \mathrm{C}$ & \begin{tabular}{|l|}
0,5 \\
\end{tabular} & 5 & 76 & 63 & & 0,08 & 0,21 & 0,51 & 0,76 & 0,00 & \begin{tabular}{|l|}
4,76 \\
\end{tabular} & \begin{tabular}{|l|}
9,40 \\
\end{tabular} & 76,22 & 44,09 \\
\hline TRIPOLI & & 56 & 5 & B & 06/10/99 & RB78-5148 & $60 . C$ & \begin{tabular}{|l|}
0,3 \\
\end{tabular} & 1 & 92 & 63 & & 0,05 & 0,04 & 0,67 & 0,76 & 0,00 & \begin{tabular}{|l|}
4,64 \\
\end{tabular} & & & \\
\hline TRIPOLI & & 56 & 6 & $\bar{A}$ & |06/10/99 & $\begin{array}{l}\text { RB78-5148 } \\
\end{array}$ & $60 . \mathrm{C}$ & \begin{tabular}{|l|}
0,3 \\
\end{tabular} & 5 & 80 & 47 & & 0,05 & 0,21 & 0,55 & 1,12 & 0,00 & \begin{tabular}{|l|}
5,85 \\
\end{tabular} & \begin{tabular}{|l|}
9,77 \\
\end{tabular} & 76,53 & 53,06 \\
\hline TRIPOLI & & 56 & 6 & B & \begin{tabular}{|l|}
$06 / 10 / 99$ \\
\end{tabular} & $\begin{array}{l}\text { RB78-5148 } \\
\end{array}$ & $60 . \mathrm{C}$ & \begin{tabular}{|l|}
0,1 \\
\end{tabular} & 3 & \begin{tabular}{|l|l}
97 \\
\end{tabular} & 51 & & $\overline{0,02}$ & 0,13 & 0,72 & 0,52 & 0,00 & \begin{tabular}{|l|}
3,92 \\
\end{tabular} & & & \\
\hline \begin{tabular}{|l|l|} 
TRIPOLI \\
\end{tabular} & & 56 & 7 & A & 06/10/99 & $\begin{array}{l}\text { RB78-5148 } \\
\end{array}$ & $60 . C$ & \begin{tabular}{|l|}
0,6 \\
\end{tabular} & 6 & 165 & 33 & & 0,10 & 0,25 & 1,00 & 0,23 & 0,00 & 4,75 & $\mid 11,31$ & 77,82 & 56,74 \\
\hline \begin{tabular}{|l|} 
TRIPOLI \\
\end{tabular} & & 56 & 7 & B & \begin{tabular}{|l|}
$06 / 10 / 99$ \\
\end{tabular} & RB78-5148 & $60 . C$ & \begin{tabular}{|l|}
0,5 \\
\end{tabular} & 1 & 170 & 42 & & 0,08 & 0,04 & 1,00 & 1,03 & 0,00 & \begin{tabular}{|l|}
6,55 \\
\end{tabular} & & & \\
\hline TRIPOLI & & 56 & 11 & A & |06/10/99 & RB78-5148 & $60 . C$ & \begin{tabular}{|l|}
0,6 \\
\end{tabular} & 6 & 182 & 51 & & 0,10 & 0,25 & 1,00 & 0,52 & 0,00 & \begin{tabular}{|l|}
5,54 \\
\end{tabular} & 12,24 & 78,61 & 40,89 \\
\hline \begin{tabular}{|l|} 
TRIPOLI \\
\end{tabular} & & 56 & 11 & $B$ & $06 / 10 / 99$ & $\begin{array}{l}\text { RB78-5148 } \\
\end{array}$ & $60 . C$ & 0,5 & 4 & 132 & 45 & & 0,08 & 0,17 & 1,00 & 1,08 & 0,00 & \begin{tabular}{|l|l|}
6,70 \\
\end{tabular} & & & \\
\hline
\end{tabular}




\begin{tabular}{|c|c|c|c|c|c|c|c|c|c|c|c|c|c|c|c|c|c|c|c|c|c|}
\hline nome & ano 2000 & & & & & & & teore & & & & & pontuaçã & & & & & & & & \\
\hline Fazenda & Solo & zona & talhão & horiz & coleta & Variedade & Estágio & K & $\mathrm{P}$ & \begin{tabular}{|lll}
$\mathrm{T}$ \\
\end{tabular} & 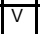 & \begin{tabular}{|l|l|} 
Arg \\
\end{tabular} & $\mathrm{K}$ & $\bar{P}$ & $T$ & V & Arg & \begin{tabular}{|l}
$N P$ \\
\end{tabular} & Ps & prod estima & prod medida \\
\hline \begin{tabular}{|l|} 
ARAQUA \\
\end{tabular} & PV-7 & 34 & 78 & A & \begin{tabular}{|l|}
$01 / 01 / 00$ \\
\end{tabular} & RB83-5486 & $30 . \mathrm{C}$ & 2,3 & 5 & 95 & 38 & & 0,38 & 0,21 & 0,70 & 0,97 & $0,0,0$ & \begin{tabular}{|l|}
9,40 \\
\end{tabular} & 16,64 & 85,00 & 45,46 \\
\hline ARAQUA & PV-8 & 34 & 78 & $B$ & $\mid 01 / 01 / 00$ & RB83-5486 & $30 . \mathrm{C}$ & 1,4 & 4 & 131 & 16 & & 0,23 & 0,17 & 1,00 & 0,11 & 0,00 & \begin{tabular}{|l|}
7,24 \\
\end{tabular} & & & \\
\hline ARAQUA & PV-9 & 34 & 80 & A & \begin{tabular}{|l|}
$01 / 01 / 00$ \\
\end{tabular} & RB83-5486 & $30 . \mathrm{C}$ & 2,7 & 3 & 128 & 37 & & 0,45 & 0,13 & 1,00 & 0,95 & 0,00 & 10,65 & 17,35 & 85,60 & 55,63 \\
\hline ARAQUA & $\mathrm{PV}-10$ & 34 & 80 & $\bar{B}$ & \begin{tabular}{|l|}
$01 / 01 / 00$ \\
\end{tabular} & RB83-5486 & $30 . \mathrm{C}$ & 1,2 & 2 & 217 & 14 & & 0,20 & 0,08 & 1,00 & 0,10 & 0,00 & \begin{tabular}{|l|}
6,70 \\
\end{tabular} & & & \\
\hline ARAQUA & $\mathrm{PV}-11$ & 34 & 81 & $\bar{A}$ & |01/01/00 & $\begin{array}{l}\text { RB85-5156 } \\
\end{array}$ & $30 . \mathrm{C}$ & 1,8 & 5 & 104 & $\mid 15$ & & 0,30 & 0,21 & 0,79 & 0,11 & 0,00 & \begin{tabular}{|l|}
6,77 \\
\end{tabular} & 12,93 & 81,86 & 60,00 \\
\hline ARAQUA & PV-12 & 34 & 81 & B & $01 / 01 / 00$ & RB85-5156 & $30 . \mathrm{C}$ & 1,2 & 2 & \begin{tabular}{|l|}
212 \\
\end{tabular} & \begin{tabular}{|l|l|}
3 \\
\end{tabular} & & 0,20 & 0,08 & 1,00 & 0,02 & \begin{tabular}{|l|}
0,00 \\
\end{tabular} & \begin{tabular}{|l|}
6,16 \\
\end{tabular} & & & \\
\hline ARAQUA & PV-13 & 34 & 83 & A & $01 / 01 / 00$ & RB83-5486 & $30 . \mathrm{C}$ & 1,3 & 3 & 37 & 8 & & 0,22 & 0,13 & 0,19 & 0,06 & 0,00 & \begin{tabular}{|l|}
4,08 \\
\end{tabular} & 8,23 & 77,88 & 88,97 \\
\hline ARAQUA & PV-14 & 34 & 83 & B & $\mid 01 / 01 / 00$ & RB83-5486 & $30 . \mathrm{C}$ & 1,2 & 4 & \begin{tabular}{|l|}
37 \\
\end{tabular} & 8 & & 0,20 & 0,17 & 0,19 & 0,06 & 0 & 4 & & & \\
\hline ARAQUA & PV-15 & 34 & 85 & $\bar{A}$ & 01/01/00 & RB83-5486 & $30 . \mathrm{C}$ & 1,5 & 2 & \begin{tabular}{|l|}
68 \\
\end{tabular} & 38 & & 0,25 & 0,08 & 0,93 & $\mid 0,97$ & 0,00 & \begin{tabular}{|l|}
9,17 \\
\end{tabular} & 17,33 & 85,58 & 76,70 \\
\hline \begin{tabular}{|l|} 
ARAQUA \\
\end{tabular} & PV-16 & 34 & 85 & B & \begin{tabular}{|l|}
$01 / 01 / 00$ \\
\end{tabular} & RB83-5486 & $30 . \mathrm{C}$ & 1,3 & 6 & \begin{tabular}{|l|}
92 \\
\end{tabular} & 58 & & 0,22 & 0,25 & 0,67 & 0,66 & 0,00 & \begin{tabular}{|l|}
8,17 \\
\end{tabular} & & & \\
\hline \begin{tabular}{|l} 
ARAQUA \\
\end{tabular} & PV-17 & 34 & 86 & A & \begin{tabular}{|l|}
$01 / 01 / 00$ \\
\end{tabular} & RB83-5486 & $30 . \mathrm{C}$ & 1,2 & 4 & \begin{tabular}{|l|}
82 \\
\end{tabular} & 12 & & 0,20 & 0,17 & 0,57 & 0,09 & 0,00 & \begin{tabular}{|l|}
5,77 \\
\end{tabular} & 13,42 & 82,27 & 69,27 \\
\hline ARAQUA & PV-18 & 34 & 86 & $B$ & 01/01/00 & RB83-5486 & $30 . \mathrm{C}$ & 1,1 & 26 & 88 & \begin{tabular}{|l|}
9 \\
\end{tabular} & & 0,18 & 0,78 & 0,63 & 0,06 & \begin{tabular}{|l|}
0,00 \\
\end{tabular} & \begin{tabular}{|l|}
7,66 \\
\end{tabular} & & & \\
\hline ARAQUA & PV-19 & 34 & 88 & $\bar{A}$ & |01/01/00 & RB83-5486 & $30 . \mathrm{C}$ & 0,8 & 5 & 38 & 34 & & 0,13 & 0,21 & 0,19 & 0,24 & 0,00 & 4,94 & 9,61 & 79,05 & 65,2 \\
\hline ARAQUA & PV-20 & 34 & 88 & B & $01 / 01 / 00$ & RB83-5486 & $30 . \mathrm{C}$ & 1,1 & 7 & 47 & 19 & & 0,18 & 0,28 & 0,24 & 0,13 & 0,00 & 4,67 & & & \\
\hline ARAQUA & $\mathrm{PV}-21$ & 34 & 89 & A & 01/01/00 & RB83-5486 & $30 . \mathrm{C}$ & 2,1 & 9 & 54 & 22 & & 0,35 & 0,33 & 0,79 & 0,16 & 0 & \begin{tabular}{|l|}
7,66 \\
\end{tabular} & 13,18 & 82,07 & 73,03 \\
\hline \begin{tabular}{|l|} 
ARAQUA \\
\end{tabular} & PV-22 & 34 & 89 & B & \begin{tabular}{|l|}
$01 / 01 / 00$ \\
\end{tabular} & RB83-5486 & $30 . \mathrm{C}$ & $\begin{array}{ll}1,7 \\
\end{array}$ & 4 & 81 & 11 & & 0,28 & 0,17 & 0,56 & 0,08 & 0,00 & \begin{tabular}{|l|}
5,51 \\
\end{tabular} & & & \\
\hline ARAQUA & PV-23 & 34 & 90 & $\bar{A}$ & 01/01/00 & RB83-5486 & $30 . \mathrm{C}$ & 1,7 & 4 & \begin{tabular}{|l|}
55 \\
\end{tabular} & \begin{tabular}{|l|}
23 \\
\end{tabular} & & 0,28 & 0,17 & 0,80 & 0,16 & 0,00 & \begin{tabular}{|l|}
6,87 \\
\end{tabular} & 13,57 & 82,40 & $42,8 \varepsilon$ \\
\hline ARAQUA & PV-24 & 34 & 90 & B & 01/01/00 & RB83-5486 & $30 . \mathrm{C}$ & 1,1 & 4 & 125 & 12 & & 0,18 & 0,17 & 1,00 & 0,09 & 0,00 & \begin{tabular}{|l|}
6,71 \\
\end{tabular} & & & \\
\hline ARAQUA & PV-25 & 34 & 91 & A & $01 / 01 / 00$ & RB83-5486 & $30 . \mathrm{C}$ & 2,4 & 3 & 67 & 22 & & 0,40 & 0,13 & 0,92 & 0,16 & 0,00 & 7,50 & 14,49 & 83,17 & 43,42 \\
\hline ARAQUA & PV-26 & 34 & 91 & B & \begin{tabular}{|l|}
$01 / 01 / 00$ \\
\end{tabular} & RB83-5486 & $30 . \mathrm{C}$ & 2,7 & 3 & 107 & 8 & & 0,45 & 0,13 & 0,82 & 0,06 & 0 & \begin{tabular}{|l|}
6,98 \\
\end{tabular} & & & \\
\hline ARAQUA & PV-27 & 34 & 92 & A & $\mid 01 / 01 / 00$ & RB83-5486 & $30 . \mathrm{C}$ & 1,3 & 4 & 42 & 40 & & 0,22 & 0,17 & 0,21 & 1,00 & 0 & \begin{tabular}{|l|}
7,41 \\
\end{tabular} & 11,50 & 80,65 & 44,93 \\
\hline ARAQUA & PV-28 & 34 & 92 & B & \begin{tabular}{|l|}
$01 / 01 / 00$ \\
\end{tabular} & RB83-5486 & $30 . \mathrm{C}$ & 1,4 & 3 & 39 & 12 & & 0,23 & 0,13 & 0,20 & 0,09 & 0,00 & \begin{tabular}{|l|}
4,09 \\
\end{tabular} & & & \\
\hline ARAQUA & PV-29 & 35 & 97 & A & \begin{tabular}{|l|}
$01 / 01 / 00$ \\
\end{tabular} & RB85-5536 & $30 . \mathrm{C}$ & 1,3 & 4 & 45 & 37 & & 0,22 & 0,17 & 0,23 & 0,95 & 0,00 & \begin{tabular}{|l|}
7,00 \\
\end{tabular} & 10,65 & 79,93 & $82,4 \varepsilon$ \\
\hline ARAQUA & PV-30 & 35 & 97 & B & 01/01/00 & RB85-5536 & $30 . \mathrm{C}$ & 0,9 & 2 & 40 & 15 & & 0,15 & 0,08 & 0,20 & 0,11 & 0,00 & \begin{tabular}{|l|}
3,64 \\
\end{tabular} & & & \\
\hline ARAQUA & PV-31 & 35 & 98 & $\bar{A}$ & |01/01/00 & $\begin{array}{l}\text { RB85-5536 } \\
\end{array}$ & $30 . \mathrm{C}$ & 1,2 & 38 & 58 & 53 & & 0,20 & 1,00 & \begin{tabular}{|l|}
0,83 \\
\end{tabular} & 0,56 & 0,00 & 10,17 & \begin{tabular}{|l|l|}
15,67 \\
\end{tabular} & 84,18 & 71,0 \\
\hline ARAQUA & PV-32 & 35 & 98 & B & $01 / 01 / 00$ & RB85-5536 & $30 . \mathrm{C}$ & 1,1 & 15 & 44 & 29 & & 0,18 & 0,50 & 0,22 & 0,21 & 0,00 & 5,50 & & & \\
\hline ARAQUA & PV-33 & 35 & 99 & A & $\mid 01 / 01 / 00$ & RB85-5536 & $30 . \mathrm{C}$ & 0,9 & 2 & 40 & 15 & & 0,15 & 0,08 & 0,20 & 0,11 & 0 & \begin{tabular}{|l|}
3,87 \\
\end{tabular} & \begin{tabular}{|l|}
9,27 \\
\end{tabular} & 78,76 & 50,62 \\
\hline ARAQUA & PV-34 & 35 & 99 & B & \begin{tabular}{|l|}
$01 / 01 / 00$ \\
\end{tabular} & RB85-5536 & $30 . \mathrm{C}$ & 0,7 & 3 & 52 & \begin{tabular}{|l|}
9 \\
\end{tabular} & & 0,12 & 0,13 & 0,77 & 0,06 & 0,00 & \begin{tabular}{|l|}
5,40 \\
\end{tabular} & & & \\
\hline ARAQUA & PV-35 & 35 & 101 & $\bar{A}$ & 01/01/00 & RB84-5257 & $30 . \mathrm{C}$ & 1,6 & 7 & 73 & 12 & & 0,27 & 0,28 & 0,98 & 0,09 & 0,00 & \begin{tabular}{|l|}
7,38 \\
\end{tabular} & 13,74 & 82,54 & 94,1 \\
\hline ARAQUA & PV-36 & 35 & 101 & B & \begin{tabular}{|l|}
$01 / 01 / 00$ \\
\end{tabular} & RB84-5257 & $30 . \mathrm{C}$ & 1,4 & 2 & 157 & 4 & & 0,23 & 0,08 & 1,00 & 0,03 & 0,00 & \begin{tabular}{|l|}
6,36 \\
\end{tabular} & & & \\
\hline ARAQUA & PV-37 & 35 & 104 & A & $01 / 01 / 00$ & RB84-5257 & $30 . \mathrm{C}$ & 1,7 & 8 & 97 & \begin{tabular}{|l|}
9 \\
\end{tabular} & & 0,28 & 0,31 & 0,72 & 0,06 & 0,00 & \begin{tabular}{|l|l|}
6,67 \\
\end{tabular} & 13,41 & 82,26 & 70,12 \\
\hline ARAQUA & PV-38 & 35 & 104 & B & $01 / 01 / 00$ & RB84-5257 & $30 . \mathrm{C}$ & 1,2 & 8 & 116 & 15 & & 0,20 & 0,31 & 0,91 & 0,11 & 0 & \begin{tabular}{|l|}
6,74 \\
\end{tabular} & & & \\
\hline ARAQUA & PV-39 & 35 & 105 & A & $\mid 01 / 01 / 00$ & RB84-5257 & $30 . \mathrm{C}$ & 2,1 & 4 & 154 & 29 & & 0,35 & 0,17 & 1,00 & 0,21 & 0 & 7,72 & \begin{tabular}{|l|}
16,87 \\
\end{tabular} & 85,19 & 64,56 \\
\hline ARAQUA & PV-40 & 35 & 105 & B & \begin{tabular}{|l|}
$01 / 01 / 00$ \\
\end{tabular} & RB84-5257 & $30 . \mathrm{C}$ & 1,3 & 3 & 171 & 36 & & 0,22 & 0,13 & 1,00 & 0,93 & 0,00 & 9,15 & & & \\
\hline \begin{tabular}{|l|} 
ARAQUA \\
\end{tabular} & $P V-41$ & 35 & 106 & A & \begin{tabular}{|l|}
$01 / 01 / 00$ \\
\end{tabular} & RB84-5257 & $30 . \mathrm{C}$ & 1,6 & 5 & 90 & 11 & & 0,27 & 0,21 & 0,65 & 0,08 & 0,00 & 6,08 & 11,52 & 80,66 & 76,03 \\
\hline ARAQUA & PV-42 & 35 & 106 & B & \begin{tabular}{|l|}
$01 / 01 / 00$ \\
\end{tabular} & RB84-5257 & $30 . \mathrm{C}$ & 1,3 & 4 & 86 & \begin{tabular}{|l|}
6 \\
\end{tabular} & & 0,22 & 0,17 & 0,61 & 0,04 & 0,00 & 5,43 & & & \\
\hline ARAQUA & PV-43 & 34 & $75 \mathrm{a}$ & $\mathrm{A}$ & 01/01/00 & $\begin{array}{l}\text { RB83-5486 } \\
\end{array}$ & 30.C & 2,6 & 8 & 74 & 13 & & 0,43 & 0,31 & 0,99 & 0,09 & 0,00 & \begin{tabular}{|l|}
8,31 \\
\end{tabular} & 15,40 & 83,95 & 61,2 \\
\hline \begin{tabular}{|l} 
ARAQUA \\
\end{tabular} & $\begin{array}{l}P V-44 \\
\end{array}$ & 34 & $75 a$ & B & $01 / 0$ & RB8 & $30 . \mathrm{C}$ & 3,6 & 4 & 91 & 12 & & 60 & 0,17 & 0,66 & 0,09 & 0,00 & 7,09 & & & \\
\hline ARAQUA & PV-45 & 34 & $76 a$ & A & $01 / 01 / 00$ & RB83-5486 & $30 . \mathrm{C}$ & 1,7 & 4 & 219 & \begin{tabular}{|c|}
6 \\
\end{tabular} & & 0,28 & 0,17 & 1,00 & 0,04 & 0 & 7,33 & \begin{tabular}{|l|l|}
13,87 \\
\end{tabular} & 82,66 & $51,5 \varsigma$ \\
\hline ARAQUA & $P V-46$ & 34 & $76 a$ & B & $\mid 01 / 01 / 00$ & RB83-5486 & $30 . \mathrm{C}$ & 1,0 & 2 & 160 & 15 & & 0,17 & 0,08 & 1,00 & 0,11 & 0 & 6,54 & & & \\
\hline AREIA BRAN & STAB & 9 & 19 & $\bar{A}$ & 01/01/00 & RB83-5054 & $20 . \mathrm{C}$ & 3,3 & 6 & 75 & 30 & & 0,55 & 0,25 & 0,50 & 0,21 & 0,00 & 10,54 & 18,79 & 86,82 & 95,13 \\
\hline AREIA BRAN & STAB & 9 & 19 & B & \begin{tabular}{|l|}
$01 / 01 / 00$ \\
\end{tabular} & RB83-5054 & $20 . \mathrm{C}$ & 1,6 & 1 & 65 & 20 & & 0,27 & 0,04 & 0,90 & 0,14 & 0,00 & 8,25 & & & \\
\hline REIA BR & STAB B & 9 & 20 & A & $01 / 01 / 00$ & RB83-5054 & $20 . \mathrm{C}$ & 4,5 & 16 & 76 & 56 & & 0,75 & 0,53 & 0,51 & 0,62 & 0,00 & 13,22 & 24,47 & 91,62 & 108,56 \\
\hline AREIA BRAN & STAB & 9 & 20 & B & 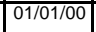 & RB83-5054 & $20 . \mathrm{C}$ & 2,8 & 5 & 66 & 62 & & 0,47 & 0,21 & 0,91 & 0,74 & \begin{tabular}{|l|}
0,00 \\
\end{tabular} & 11,25 & & & \\
\hline AREIA BRAN & STA B & 9 & 21 & A & $\mid 01 / 01 / 00$ & RB83-5054 & $20 . \mathrm{C}$ & 2,5 & 23 & 73 & 20 & & 0,42 & 0,70 & 0,98 & 0,14 & 0 & 12,72 & 20,91 & 88,61 & 110,78 \\
\hline AREIA BRAN & STAB & 9 & 21 & B & $01 / 01 / 00$ & RB83-5054 & $20 . \mathrm{C}$ & 1,6 & 3 & 57 & 17 & & 0,27 & 0,13 & 0,82 & 0,12 & 0,00 & 8,20 & & & \\
\hline AREIA BRAN & STAB & 2 & 24 & $\mathrm{~A}$ & 01/01/00 & $\begin{array}{l}\text { SP80-1842 } \\
\end{array}$ & $20 . \mathrm{C}$ & 4,3 & 9 & 70 & 51 & & 0,72 & 0,33 & 0,95 & 0,52 & 0,00 & 11,99 & 22,59 & 90,03 & 83,43 \\
\hline AREIA BRAN & STAB & 2 & 24 & $\bar{B}$ & \begin{tabular}{|l|}
$01 / 01 / 00$ \\
\end{tabular} & SP80-1842 & $20 . \mathrm{C}$ & 2,8 & 4 & 70 & 55 & & 0,47 & 0,17 & 0,95 & 0,60 & 0 & 10,60 & & & \\
\hline AREIA BRAN & STAB & 2 & 33 & A & 01/01/00 & RB80-6043 & $20 . \mathrm{C}$ & 2,1 & 4 & 64 & 26 & & 0,35 & 0,17 & 0,89 & 0,18 & 0,00 & \begin{tabular}{|l|}
10,77 \\
\end{tabular} & 19,43 & 87,36 & 100,7 \\
\hline AREIA BRAN & STA B & 2 & 33 & B & $01 / 01 / 00$ & $\begin{array}{l}\text { RB80-6043 } \\
\end{array}$ & $20 . \mathrm{C}$ & \begin{tabular}{|l|}
1,7 \\
\end{tabular} & 2 & 68 & 30 & & 0,28 & 0,08 & 0,93 & 0,21 & 0,00 & 8,65 & & & \\
\hline \begin{tabular}{|l} 
AREIA BRAN \\
\end{tabular} & STAB & 2 & 35 & A & $01 / 01 / 00$ & $\begin{array}{l}\text { SP80-1842 } \\
\end{array}$ & $20 . \mathrm{C}$ & 2,1 & 6 & 111 & 20 & & 0,35 & 0,25 & 0,86 & 0,14 & 0,00 & 10,81 & 21,23 & 88,88 & 89,64 \\
\hline AREIA BRAN & STA B & 2 & 35 & B & \begin{tabular}{|l|}
$01 / 01 / 00$ \\
\end{tabular} & SP80-1842 & $20 . C$ & \begin{tabular}{|l|}
2,4 \\
\end{tabular} & 3 & 100 & 28 & & 0,40 & 0,13 & 0,75 & 0,20 & 0 & 10,42 & & & \\
\hline AREIA BRAN & STAB & 2 & 36 & $\bar{A}$ & \begin{tabular}{|l|}
$01 / 01 / 00$ \\
\end{tabular} & $\begin{array}{l}\text { SP80-1842 } \\
\text { (1) }\end{array}$ & $20 . \mathrm{C}$ & \begin{tabular}{|l|}
3,9 \\
\end{tabular} & 8 & 92 & 36 & & 0,65 & 0,31 & \begin{tabular}{|l|}
0,67 \\
\end{tabular} & 0,93 & 0,00 & 13,68 & 26,74 & 93,55 & 92,7 \\
\hline \begin{tabular}{|l|} 
AREIA BRAN \\
\end{tabular} & STAB & 2 & 36 & $\bar{B}$ & \begin{tabular}{|l|}
$01 / 01 / 00$ \\
\end{tabular} & SP80-1842 & $20 . \mathrm{C}$ & 4,1 & 4 & 82 & 36 & & 0,68 & 0,17 & 0,57 & 0,93 & 0,00 & 13,06 & & & \\
\hline \begin{tabular}{|l|} 
AREIA BRAN \\
\end{tabular} & STAB & 6 & 55 & $\bar{A}$ & $01 / 01 / 00$ & $\begin{array}{l}\text { RB83-5486 } \\
\end{array}$ & $30 . \mathrm{C}$ & \begin{tabular}{|l|}
1,5 \\
\end{tabular} & 5 & 54 & 59 & & 0,25 & 0,21 & 0,79 & 0,68 & 0,00 & 8,86 & 17,91 & 86,07 & 79,12 \\
\hline AREIA BRAN & STA B & 6 & 55 & B & \begin{tabular}{|l|}
$01 / 01 / 00$ \\
\end{tabular} & RB83-5486 & $30 . \mathrm{C}$ & 1,3 & 6 & 57 & \begin{tabular}{|l|}
64 \\
\end{tabular} & & 0,22 & 0,25 & 0,82 & 0,78 & 0 & 9,05 & & & \\
\hline AREIA BRAN & STA B & 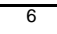 & 56 & $\bar{A}$ & $\mid 01 / 01 / 00$ & RB83-5486 & $30 . \mathrm{C}$ & 1,8 & 3 & 53 & 41 & & 0,30 & 0,13 & 0,78 & 1,02 & 0 & 9,44 & 17,42 & 85,66 & 51,9 \\
\hline AREIA BRAN & STAB & 6 & 56 & B & \begin{tabular}{|l|}
$01 / 01 / 00$ \\
\end{tabular} & 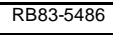 & $30 . \mathrm{C}$ & \begin{tabular}{|l|}
1,8 \\
\end{tabular} & 3 & 52 & 57 & & 0,30 & 0,13 & 0,77 & 0,64 & 0,00 & 7,98 & & & \\
\hline \begin{tabular}{|l|} 
AREIA BRAN \\
\end{tabular} & STA B & 6 & 57 & A & $01 / 01 / 00$ & $\begin{array}{l}\text { RB83-5486 } \\
\end{array}$ & $30 . \mathrm{C}$ & \begin{tabular}{|l|}
1,6 \\
\end{tabular} & 4 & 45 & 44 & & 0,27 & 0,17 & 0,23 & 1,07 & 0 & 7,95 & 14,87 & 83,50 & 69,74 \\
\hline AREIA BRAN & STA B & 6 & 57 & B & 01/01/00 & RB83-5486 & $30 . \mathrm{C}$ & \begin{tabular}{|l|}
1,7 \\
\end{tabular} & 1 & 44 & 36 & & 0,28 & 0,04 & 0,22 & 0,93 & 0,00 & 6,91 & & & \\
\hline REIA BRAN & STAB & 7 & 85 & $\bar{A}$ & Dotiotion & RB72-454 & $30 . \mathrm{C}$ & \begin{tabular}{|l|}
0,7 \\
\end{tabular} & 4 & 55 & \begin{tabular}{|l|}
5 \\
\end{tabular} & & 0,12 & 0,17 & 0,80 & 0,04 & 0,00 & \begin{tabular}{|l|}
5,76 \\
\end{tabular} & 9,97 & 79,35 & 85,3 \\
\hline AREIA BRAN & STA B & 7 & 85 & B & $01 / 01 / 00$ & $\begin{array}{l}\text { RB72-454 } \\
\end{array}$ & $30 . \mathrm{C}$ & \begin{tabular}{|l|}
0,9 \\
\end{tabular} & 5 & 32 & 12 & & 0,15 & 0,21 & 0,16 & 0,09 & 0,00 & 4,21 & & & \\
\hline AREIA BRAN & STAB & 77 & 86 & $\bar{A}$ & $01 / 01 / 00$ & RB72-454 & $40 . \mathrm{C}$ & 1,0 & 7 & 56 & 16 & & 0,17 & 0,28 & 0,81 & 0,11 & 0,00 & 5,60 & 8,86 & 78,41 & 67,13 \\
\hline REIA BRAN & STA B & 7 & 86 & B & $01 / 01 / 00$ & RB72-454 & $40 . \mathrm{C}$ & \begin{tabular}{|l|}
0,9 \\
\end{tabular} & 4 & 35 & 20 & & 0,15 & 0,17 & $0,1 \varepsilon$ & 0,14 & 0 & 3,25 & & & \\
\hline AREIA BRAN & STAB & 7 & 90 & $\bar{A}$ & \begin{tabular}{|l|}
$01 / 01 / 00$ \\
\end{tabular} & RB72-454 & $30 . C$ & 1,7 & 6 & 100 & 20 & & 0,28 & 0,25 & 0,7 & 0,14 & 0,00 & 9,08 & 15,71 & 84,21 & 91,7 \\
\hline
\end{tabular}




\begin{tabular}{|c|c|c|c|c|c|c|c|c|c|c|c|c|c|c|c|c|c|c|c|c|c|}
\hline nome & & & & & & & & teores & & & & & pontuação & & & & & & & & \\
\hline Fazenda & Solo & zona & talhão & horiz & coleta & Variedade & Estágio & $\mathrm{K}$ & $\bar{P}$ & $\mathrm{~T}$ & $\mathrm{~V}$ & Arg & $\mathrm{K}$ & $P$ & \begin{tabular}{|l|l|}
$T$ \\
\end{tabular} & $\mathrm{~V}$ & Arg & $\mathrm{NP}$ & \begin{tabular}{|l|} 
Ps \\
\end{tabular} & prod estima & prod medida \\
\hline \begin{tabular}{|l|} 
AREIA BRAN \\
\end{tabular} & STAB & 7 & 90 & $\bar{B}$ & $01 / 01 / 00$ & RB72-454 & $30 . \mathrm{C}$ & 0,9 & 4 & 108 & 9 & & 0,15 & 0,17 & 0,83 & 0,06 & 0,00 & 6,63 & & & \\
\hline AREIA BRAN & STAB & 7 & 92 & A & $01 / 01 / 00$ & RB72-454 & 30.C & 1,2 & 4 & 49 & 14 & & 0,20 & 0,17 & 0,25 & 0,10 & 0,00 & 4,98 & 10,61 & 79,90 & 57,39 \\
\hline \begin{tabular}{|l|} 
AREIA BRAN \\
\end{tabular} & STAB & 7 & 92 & B & $01 / 01 / 00$ & RB72-454 & $30 . \mathrm{C}$ & 0,7 & 3 & 56 & 7 & & 0,12 & 0,13 & \begin{tabular}{|l|}
0,81 \\
\end{tabular} & 0,05 & 0,000 & \begin{tabular}{|l|}
5,63 \\
\end{tabular} & & & \\
\hline B.J.MENEGH & PV9 & 57 & 1 & A & $01 / 01 / 00$ & RB72-454 & $40 . \mathrm{C}$ & 3,6 & 13 & 135 & 77 & 21 & 0,60 & 0,44 & \begin{tabular}{|l|}
1,00 \\
\end{tabular} & 1,00 & 0,42 & 12,28 & 23,47 & 90,78 & 65,55 \\
\hline B.J.MENEGH & PV9 & 57 & 1 & B & 01/01/00 & RB72-454 & $40 . \mathrm{C}$ & 2,3 & 11 & 130 & 55 & 43 & 0,38 & 0,39 & $\mid 1,00$ & 0,60 & 0,86 & 11,20 & & & \\
\hline B.VISTA SH & & 1 & 17 & $\mathrm{~A}$ & $01 / 01 / 00$ & SP80-1842 & $40 . C$ & 1,8 & 7 & 63 & 39 & & 0,30 & 0,28 & 0,88 & 0,98 & 0,00 & \begin{tabular}{|l|}
9,28 \\
\end{tabular} & 15,24 & 83,81 & 95,67 \\
\hline B.VISTA SH & & 1 & 17 & $\bar{B}$ & $01 / 01 / 00$ & $\begin{array}{l}\text { SP80-1842 } \\
\text { (1) }\end{array}$ & $40 . \mathrm{C}$ & 1,4 & 4 & 60 & 30 & & 0,23 & 0,17 & 0,85 & 0,21 & 0,00 & \begin{tabular}{|l|}
5,96 \\
\end{tabular} & & & \\
\hline B.VISTA SH & & 1 & 18 & $\mathrm{~A}$ & $01 / 01 / 00$ & $\begin{array}{l}\text { SP80-1842 } \\
\text { (1) }\end{array}$ & $40 . \mathrm{C}$ & 1,7 & 5 & 78 & 33 & & 0,28 & 0,21 & 0,53 & 0,23 & 0,00 & 7,59 & 13,46 & 82,31 & $\overline{104,23}$ \\
\hline B.VISTA SH & & 1 & 18 & B & 01/01/00 & SP80-1842 & $40 . \mathrm{C}$ & 0,8 & 3 & 73 & 20 & & 0,13 & 0,13 & \begin{tabular}{|l|}
0,98 \\
\end{tabular} & 0,14 & 0,000 & \begin{tabular}{|l|l|}
5,87 \\
\end{tabular} & & & \\
\hline B.VISTA SH & & 1 & 19 & A & $01 / 01 / 00$ & SP80-1842 & $40 . \mathrm{C}$ & 1,4 & 4 & 75 & 14 & & 0,23 & $\mid 0,17$ & 0 & 0,10 & 0,00 & \begin{tabular}{|l|}
5,02 \\
\end{tabular} & \begin{tabular}{|l|}
9,58 \\
\end{tabular} & 79,02 & 81,3 \\
\hline B.VISTA SH & & 1 & 19 & $\mathrm{~B}$ & 01/01/00 & SP80-1842 & $40 . \mathrm{C}$ & 0,9 & 3 & 88 & 9 & & 0,15 & 0,13 & 0,63 & 0,06 & 0,00 & \begin{tabular}{|l|}
4,56 \\
\end{tabular} & & & \\
\hline B.VISTA SH & & 1 & 21 & A & $01 / 01 / 00$ & SP80-1842 & $40 . \mathrm{C}$ & 1,7 & 6 & 96 & 16 & & 0,28 & 0,25 & 0,71 & 0,11 & 0,00 & 7,90 & 12,91 & 81,84 & 112,57 \\
\hline B.VISTA SH & & 1 & 21 & $\bar{B}$ & $01 / 01 / 00$ & 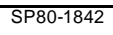 & $40 . \mathrm{C}$ & 1,0 & 3 & 90 & 11 & & 0,17 & 0,13 & 0,65 & 0,08 & 0,00 & \begin{tabular}{|l|}
5,01 \\
\end{tabular} & & & \\
\hline B.VISTA SH & & 1 & 24 & A & $01 / 01 / 00$ & 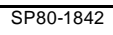 & $40 . \mathrm{C}$ & 2,0 & 4 & 68 & 23 & & 0,33 & 0,17 & \begin{tabular}{|l|}
0,93 \\
\end{tabular} & 0,16 & 0,00 & \begin{tabular}{|l|}
6,58 \\
\end{tabular} & 12,95 & 81,88 & 103,6 \\
\hline B.VISTA SH & & 1 & 24 & B & $01 / 01 / 00$ & SP80-1842 & $40 . \mathrm{C}$ & 3,8 & 1 & 60 & 21 & & 0,63 & 0,04 & \begin{tabular}{|l|}
0,85 \\
\end{tabular} & 0,15 & 0,00 & \begin{tabular}{|l|l|}
6,37 \\
\end{tabular} & & & \\
\hline B.VISTA SH & & 1 & 26 & A & $01 / 01 / 00$ & SP80-1842 & $40 . \mathrm{C}$ & 1,9 & 4 & 89 & 10 & & 0,32 & 0,17 & \begin{tabular}{|l|}
0,64 \\
\end{tabular} & 0,07 & 0,00 & \begin{tabular}{|l|}
5,68 \\
\end{tabular} & 10,32 & 79,65 & 78,1 \\
\hline B.VISTA SH & & 1 & 26 & B & $01 / 01 / 00$ & SP80-1842 & $40 . C$ & 0,8 & 3 & 85 & 5 & & 0,13 & 0,13 & 0,60 & 0,04 & 0 & 4,63 & & & \\
\hline B.VISTA SH & & 4 & 62 & A & $01 / 01 / 00$ & SP80-1842 & $30 . \mathrm{C}$ & 2,6 & 7 & 99 & 47 & & 0,43 & 0,28 & 0,74 & 1,12 & 0,00 & 12,51 & 19,96 & 87,81 & 117,45 \\
\hline B.VISTA SH & & 4 & 62 & $\bar{B}$ & $01 / 01 / 00$ & 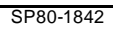 & $30 . \mathrm{C}$ & 0,8 & 4 & 75 & 58 & & 0,13 & 0,17 & 0,50 & 0,66 & 0,00 & \begin{tabular}{|l|}
7,46 \\
\end{tabular} & & & \\
\hline B.VISTA SH & & 4 & 64 & $\mathrm{~A}$ & $01 / 01 / 00$ & $\begin{array}{l}\text { SP80-1842 } \\
\text {. }\end{array}$ & $30 . \mathrm{C}$ & 3,0 & 4 & 110 & 27 & & 0,50 & 0,17 & 0,85 & 0,19 & 0,00 & 9,93 & 21,30 & 88,94 & 72,4 \\
\hline B.VISTA SH & & 4 & 64 & $B$ & $01 / 01 / 00$ & $\begin{array}{l}\text { SP80-1842 } \\
\end{array}$ & $30 . \mathrm{C}$ & 2,7 & 2 & 94 & 38 & & 0,45 & 0,08 & \begin{tabular}{|l|l|}
0,69 \\
\end{tabular} & 0,97 & 0,00 & \begin{tabular}{|l|}
11,37 \\
\end{tabular} & & & \\
\hline B.VISTA SH & & 4 & 66 & A & $01 / 01 / 00$ & SP80-1842 & $30 . \mathrm{C}$ & 2,1 & 34 & 113 & 13 & & 0,35 & 0,98 & 0,88 & 0,09 & 0,00 & \begin{tabular}{|l|}
11,69 \\
\end{tabular} & 20,94 & 88,63 & 62,5 \\
\hline B.VISTA SH & & 4 & 66 & B & $01 / 01 / 00$ & SP80-1842 & $30 . \mathrm{C}$ & 1,7 & 5 & 114 & 14 & & 0,28 & 0,21 & 0,89 & 0,10 & 0,00 & \begin{tabular}{l|l}
9,24 \\
\end{tabular} & & & \\
\hline B.VISTA SH & & 4 & 69 & A & $01 / 01 / 00$ & SP80-1842 & $30 . \mathrm{C}$ & 3,0 & 10 & 111 & 42 & & 0,50 & 0,36 & 0,86 & 1,03 & 0,00 & \begin{tabular}{|l|}
13,07 \\
\end{tabular} & 22,24 & 89,74 & 101,4 \\
\hline B.VISTA SH & & 4 & 69 & $B$ & $01 / 01 / 00$ & $\begin{array}{l}\text { SP80-1842 } \\
\end{array}$ & $30 . \mathrm{C}$ & 0,7 & 7 & 108 & 33 & & 0,12 & 0,28 & 0,83 & 0,23 & 0,00 & \begin{tabular}{|l|}
9,18 \\
\end{tabular} & & & \\
\hline B.VISTA SH & & 4 & 70 & A & $01 / 01 / 00$ & SP80-1842 & $30 . \mathrm{C}$ & 2,1 & 8 & 112 & 42 & & 0,35 & 0,31 & 0,87 & 1,03 & 0,00 & 12,48 & 23,99 & 91,22 & $\overline{103,12}$ \\
\hline B.VISTA SH & & 4 & 70 & B & $01 / 01 / 00$ & 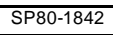 & $30 . \mathrm{C}$ & 0,8 & 5 & 111 & 42 & & 0,13 & 0,21 & \begin{tabular}{|l|}
0,86 \\
\end{tabular} & 1,03 & 0,00 & $\mid$\begin{tabular}{|l|}
11,51 \\
\end{tabular} & & & \\
\hline B.VISTA SH & & 4 & 74 & A & $01 / 01 / 00$ & SP80-1842 & $30 . \mathrm{C}$ & 2,3 & 6 & 83 & 30 & & 0,38 & 0,25 & 0,58 & 0,21 & 0,00 & \begin{tabular}{|l|}
9,08 \\
\end{tabular} & 18,90 & 86,91 & $\overline{103,4}$ \\
\hline B.VISTA SH & & $\overline{4}$ & 74 & B & $01 / 01 / 00$ & SP80-1842 & $30 . \mathrm{C}$ & 1,9 & 4 & 63 & 39 & & 0,32 & 0,17 & \begin{tabular}{|c|}
0,88 \\
\end{tabular} & 0,98 & 0,00 & \begin{tabular}{|l|}
9,82 \\
\end{tabular} & & & \\
\hline B.VISTA SH & & 4 & 75 & A & $01 / 01 / 00$ & SP80-1816 & $30 . \mathrm{C}$ & 3,6 & 5 & 77 & 32 & & 0,60 & 0,21 & 0,52 & 0,23 & 0,00 & 9,47 & 16,86 & 85,19 & 98,8 \\
\hline B.VISTA SH & & 4 & 75 & B & $01 / 01 / 00$ & SP80-1816 & $30 . \mathrm{C}$ & 1,7 & 7 & 103 & 14 & & 0,28 & 0,28 & 0,78 & 0,10 & 0,00 & 7,40 & & & \\
\hline B.VISTA SH & & 4 & 76 & $\mathrm{~A}$ & $01 / 01 / 00$ & $\begin{array}{l}\text { SP80-1816 } \\
\end{array}$ & $30 . \mathrm{C}$ & 3,3 & 20 & 69 & 39 & & 0,55 & 0,63 & 0,94 & 0,98 & 0,00 & 12,52 & 19,24 & 87,19 & 79,7 \\
\hline B.VISTA SH & & 4 & 76 & $\bar{B}$ & $01 / 01 / 00$ & $\begin{array}{l}\text { SP80-1816 } \\
\end{array}$ & $30 . \mathrm{C}$ & 1,4 & 4 & 102 & 13 & & 0,23 & 0,17 & 0,77 & 0,09 & 0,00 & 6,71 & & & \\
\hline B.VISTA SH & & 7 & 117 & A & $01 / 01 / 00$ & SP80-1842 & $30 . \mathrm{C}$ & 2,2 & 7 & 118 & 45 & & 0,37 & 0,28 & 0,93 & 1,08 & 0,00 & 12,78 & 23,45 & 90,76 & 75,85 \\
\hline B.VISTA SH & & 7 & 117 & B & $01 / 01 / 00$ & SP80-1842 & $30 . \mathrm{C}$ & 0,6 & 3 & 98 & 40 & & 0,10 & 0,13 & 0,73 & 1,00 & 0,00 & 10,67 & & & \\
\hline B.VISTA SH & & 7 & 119 & A & $01 / 01 / 00$ & SP80-1842 & $30 . \mathrm{C}$ & 1,8 & 12 & 125 & 21 & & 0,30 & 0,42 & 1,00 & 0,15 & 0,00 & 10,40 & 19,66 & 87,55 & $\overline{112,32}$ \\
\hline B.VISTA SH & & 7 & 119 & B & $01 / 01 / 00$ & SP80-1842 & $30 . \mathrm{C}$ & 1,4 & 5 & 114 & 22 & & 0,23 & 0,21 & 0,89 & 0,16 & 0,00 & 9,26 & & & \\
\hline BOM RETIRO & PE & 2 & 13 & A & 01/01/00 & RB83-5486 & $30 . \mathrm{C}$ & 1,7 & 8 & 51 & 33 & & 0,28 & 0,31 & 0,76 & 0,23 & 0,00 & 7,60 & 16,06 & 84,51 & 84,4 \\
\hline BOM RETIRO & $\mathrm{PE}$ & 2 & 13 & B & $01 / 01 / 00$ & $\begin{array}{l}\text { RB83-5486 } \\
\end{array}$ & $30 . \mathrm{C}$ & \begin{tabular}{|l}
0,7 \\
\end{tabular} & 2 & 52 & 40 & & 0,12 & 0,08 & \begin{tabular}{|l|l|}
0,77 \\
\end{tabular} & 1,00 & 0,00 & 8,46 & & & \\
\hline \begin{tabular}{|l|} 
BOM RETIRO \\
\end{tabular} & $\mathrm{PE}$ & 2 & 14 & A & $01 / 01 / 00$ & $\begin{array}{l}\text { RB83-5486 } \\
\end{array}$ & $30 . \mathrm{C}$ & 2,1 & 7 & 59 & 47 & & 0,35 & 0,28 & 0,84 & 1,12 & 0,00 & 10,76 & 18,54 & 86,60 & 79,30 \\
\hline BOM RETIRO & $\mathrm{PE}$ & 2 & 14 & B & $01 / 01 / 00$ & RB83-5486 & $30 . \mathrm{C}$ & 0,8 & 3 & 52 & 57 & & 0,13 & 0,13 & 0,77 & 0,64 & 0,00 & 7,78 & & & \\
\hline BOM RETIRO & PE & 2 & 16 & A & $01 / 01 / 00$ & RB83-5486 & $30 . \mathrm{C}$ & 1,1 & 10 & 67 & 37 & & 0,18 & 0,36 & 0,92 & 0,95 & 0 & 10,40 & 17,39 & 85,63 & 86,42 \\
\hline BOM RETIRO & PE & 2 & 16 & B & $01 / 01 / 00$ & RB83-5486 & $30 . \mathrm{C}$ & 0,9 & 6 & 52 & 26 & & 0,15 & 0,25 & 0,77 & 0,18 & 0,00 & 6,99 & & & \\
\hline \begin{tabular}{|l|} 
CONC.OURO \\
\end{tabular} & & 1 & 1 & A & | & RB85-5156 & $30 . \mathrm{C}$ & 2,3 & 5 & 72 & 56 & & 0,38 & 0,21 & \begin{tabular}{|l|}
0,97 \\
\end{tabular} & 0,62 & 0,00 & 11,35 & 20,56 & 88,31 & 123,00 \\
\hline \begin{tabular}{|l|} 
CONC.OURO \\
\end{tabular} & & 1 & 1 & $\bar{B}$ & $01 / 01 / 00$ & $\begin{array}{l}\text { RB85-5156 } \\
\end{array}$ & $30 . \mathrm{C}$ & 0,4 & 3 & 62 & 45 & & 0,07 & 0,13 & 0,87 & 1,08 & 0,00 & 9,21 & & & \\
\hline \begin{tabular}{|l|} 
CONC.OURO \\
\end{tabular} & & 1 & 3 & A & $01 / 01 / 00$ & RB85-5156 & $30 . \mathrm{C}$ & 1,5 & 4 & 45 & 60 & & 0,25 & 0,17 & \begin{tabular}{|l|}
0,23 \\
\end{tabular} & 0,70 & 0,00 & \begin{tabular}{|l|l|}
6,73 \\
\end{tabular} & 11,29 & 80,47 & 79,76 \\
\hline $\begin{array}{l}\text { CONC.OURO } \\
\end{array}$ & & 1 & 3 & B & $01 / 01 / 00$ & RB85-5156 & $30 . \mathrm{C}$ & 0,9 & 2 & 37 & 32 & & 0,15 & 0,08 & 0,19 & 0,23 & 0,00 & 4,56 & & & \\
\hline COVETINGA & $\mathrm{PE}$ & 20 & 7 & A & | & RB72-454 & $40 . C$ & 6,8 & 3 & 96 & 39 & & 1,00 & 0,13 & 0,71 & 0,98 & 0 & 12,28 & 19,87 & 87,73 & 78,53 \\
\hline COVETINGA & $\mathrm{PE}$ & 20 & 7 & $\mathrm{~B}$ & 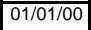 & RB72-454 & $40 . C$ & 5,7 & 2 & 85 & 31 & & 0,95 & 0,08 & 0,60 & 0,22 & 0,00 & 7,59 & & & \\
\hline COVETINGA & PE & 20 & 8 & A & $01 / 01 / 00$ & RB72-454 & $40 . C$ & 3,5 & 7 & 93 & 37 & & 0,58 & 0,28 & 0,68 & 0,95 & 0,00 & 11,30 & 21,12 & 88,78 & 113,56 \\
\hline COVETINGA & $\mathrm{PE}$ & 20 & 0 & B & $01 / 01 / 00$ & RB72-454 & $40 . \mathrm{C}$ & 1,5 & 3 & 87 & 40 & & 0,25 & 0,13 & \begin{tabular}{|l|}
0,62 \\
\end{tabular} & 1,00 & 0,00 & 9,81 & & & \\
\hline COVETINGA & $\mathrm{PE}$ & 20 & 10 & A & $01 / 01 / 00$ & $\begin{array}{l}\text { RB72-454 } \\
\end{array}$ & $50 . \mathrm{C}$ & 1,7 & 4 & 100 & 53 & & 0,28 & 0,17 & 0,75 & 0,56 & 0,00 & 8,13 & 15,31 & 83,87 & 90,60 \\
\hline COVETINGA & PE & 20 & 10 & B & $01 / 01 / 00$ & RB72-454 & $50 . \mathrm{C}$ & 1,1 & 2 & 72 & 69 & & 0,18 & 0,08 & 0,97 & 0,88 & 0,00 & 7,18 & & & \\
\hline COVETINGA & PE & 20 & 11 & A & $01 / 01 / 00$ & RB72-454 & $50 . \mathrm{C}$ & 5,2 & 3 & 98 & 46 & & 0,87 & 0,13 & 0,73 & 1,10 & 0,00 & 11,32 & 21,11 & 88,77 & 99,5 \\
\hline COVETINGA & $\mathrm{PE}$ & 20 & 11 & $\bar{B}$ & $01 / 01 / 00$ & RB72-454 & $50 . \mathrm{C}$ & 5,8 & 3 & 81 & 58 & & 0,97 & 0,13 & 0,56 & 0,66 & 0,00 & 9,79 & & & \\
\hline MONTE BELO & & 1 & 2 & A & $01 / 01 / 00$ & RB83-5486 & $30 . \mathrm{C}$ & 1,1 & 39 & 46 & 32 & & 0,18 & 1,00 & 0,23 & 0,23 & 0,00 & 7,55 & 13,77 & 82,57 & 81,85 \\
\hline MONTE BELO & & 1 & 2 & B & 01/01/00 & RB83-5486 & $30 . C$ & 1,1 & 4 & 56 & 16 & & 0,18 & 0,17 & 0,81 & 0,11 & 0,00 & 6,22 & & & \\
\hline MONTE BELO & & 1 & 3 & A & $01 / 01 / 00$ & 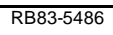 & $30 . \mathrm{C}$ & 2,1 & 161 & 79 & 64 & & 0,35 & 1,00 & 0,54 & 0,78 & 0,00 & 11,09 & 18,33 & $\overline{86,43}$ & 67,7 \\
\hline MONTE BELO & & 1 & 3 & $B$ & 01/01/00 & RB83-5486 & $30 . \mathrm{C}$ & 0,8 & 25 & 75 & 22 & & 0,13 & 0,75 & 0,50 & 0,16 & 0,00 & 7,24 & & & \\
\hline MONTE BELO & & 1 & 4 & A & $01 / 01 / 00$ & $\begin{array}{l}\text { RB83-5486 } \\
\end{array}$ & $30 . \mathrm{C}$ & 0,9 & 10 & 47 & 34 & & 0,15 & 0,36 & 0,24 & 0,24 & 0,00 & 5,74 & 12,16 & 81,21 & 60,37 \\
\hline MONTE BELO & & 1 & 4 & B & $01 / 01 / 00$ & $\begin{array}{ll}\text { RB83-5486 } \\
\end{array}$ & $30 . \mathrm{C}$ & \begin{tabular}{|l|}
0,7 \\
\end{tabular} & 7 & 53 & 20 & & 0,12 & 0,28 & 0,78 & 0,14 & 0 & 6,42 & & & \\
\hline MONTE BELO & & 1 & 5 & A & | $01 / 01 / 00$ & RB83-5486 & $30 . C$ & 1,2 & 6 & 56 & 16 & & 0,20 & 0,25 & 0,81 & 0,11 & 0,00 & 6,75 & 11,53 & 80,68 & 68,57 \\
\hline MONTE BELO & & 1 & 5 & B & $01 / 01 / 00$ & RB83-5486 & $30 . \mathrm{C}$ & 1,0 & 10 & 45 & 6 & & 0,17 & 0,36 & 0,23 & 0,04 & 0,00 & 4,79 & & & \\
\hline MONTE BELO & & 1 & 6 & A & $01 / 01 / 00$ & 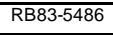 & $30 . \mathrm{C}$ & 0,9 & 56 & 68 & 23 & & 0,15 & 1,00 & 0,93 & 0,16 & 0,00 & 9,58 & 15,74 & 84,24 & 54,81 \\
\hline MONTE BELO & & 1 & 6 & B & $01 / 01 / 00$ & $\begin{array}{l}\text { RB83-5486 } \\
\end{array}$ & $30 . \mathrm{C}$ & \begin{tabular}{|l|l|}
0,7 \\
\end{tabular} & 7 & 51 & 1 & & 0,12 & 0,28 & 0,76 & 0,05 & 0,00 & 6,16 & & & \\
\hline MONTE BELO & & 1 & 8 & A & |01/01/00 & RBB85-5035 & 30.C & 0,9 & 5 & 47 & 100 & & 0,15 & 0,21 & 0,24 & $|0,07|$ & 0,00 & 4,39 & 8,20 & 77,85 & 65,8 \\
\hline DNTE BELO & & 1 & 8 & B & $01 / 01 / 00$ & RB85-5035 & $30 . \mathrm{C}$ & 0,6 & 3 & 45 & 6 & & 0,10 & 0,13 & 0,23 & 0,04 & 0 & 3,80 & & & \\
\hline IONTE BELO & & 2 & 11 & $\mathrm{~A}$ & $01 / 01 / 00$ & $\begin{array}{l}\text { RB85-5035 } \\
\end{array}$ & $30 . \mathrm{C}$ & \begin{tabular}{|l|}
0,6 \\
\end{tabular} & 3 & 52 & 26 & & 0,10 & 0,13 & 0,77 & 0,18 & 0,00 & 6,24 & 12,12 & 81,17 & 92,7 \\
\hline
\end{tabular}


82

\begin{tabular}{|c|c|c|c|c|c|c|c|c|c|c|c|c|c|c|c|c|c|c|c|c|c|}
\hline nome & & & & & & & & teores & & & & & pontuação & & & & & & & & \\
\hline Fazenda & Solo & zona & talhão & horiz & coleta & Variedade & Estágio & $\bar{K}$ & $P$ & \begin{tabular}{|lll}
$T$ \\
\end{tabular} & \begin{tabular}{|l|l}
$\mathrm{v}$ \\
\end{tabular} & Arg & $\mathrm{K}$ & $P$ & $T$ & $\mathrm{v}$ & \begin{tabular}{|l|} 
Arg \\
\end{tabular} & \begin{tabular}{|l|}
$N P$ \\
\end{tabular} & \begin{tabular}{|l|l|} 
Ps \\
\end{tabular} & od estima & \begin{tabular}{|l|} 
prod medida \\
\end{tabular} \\
\hline MONTE BELO & & 2 & 11 & $\bar{B}$ & 01/01/00 & RB85-5035 & $30 . \mathrm{C}$ & 1,0 & 2 & 52 & \begin{tabular}{l|l}
9 \\
\end{tabular} & & 0,17 & 0,08 & 0,77 & 0,06 & 0,00 & 5,88 & & & \\
\hline ONTE BELO & & 2 & 12 & A & $01 / 01 / 00$ & $\begin{array}{l}\text { RB83-5486 } \\
\end{array}$ & $30 . \mathrm{C}$ & 0,8 & 8 & \begin{tabular}{|l|}
53 \\
\end{tabular} & \begin{tabular}{|l|}
35 \\
\end{tabular} & & 0,13 & 0,31 & 0,78 & 0,92 & 0,00 & \begin{tabular}{|l|}
9,48 \\
\end{tabular} & 15,61 & 84,13 & 70,33 \\
\hline MONTE BELO & & 2 & 12 & B & $01 / 01 / 00$ & RB83-5486 & $30 . \mathrm{C}$ & 1,0 & 3 & \begin{tabular}{|l|}
54 \\
\end{tabular} & 12 & & 0,17 & 0,13 & 0,79 & 0,09 & 0,00 & 6,13 & & & \\
\hline IONTE BELO & & 3 & 32 & A & 01/01/00 & RB85-5035 & $30 . \mathrm{C}$ & 0,3 & 5 & 50 & 24 & & 0,05 & 0,21 & 0,75 & 0,17 & 0,00 & 6,39 & 12,23 & 81,26 & 72,78 \\
\hline MONTE BELO & & 3 & 32 & $B$ & $01 / 01 / 00$ & RB85-5035 & $30 . \mathrm{C}$ & 0,5 & 3 & 50 & \begin{tabular}{|l|}
16 \\
\end{tabular} & & 0,08 & 0,13 & 0,75 & 0,11 & 0,00 & 5,84 & & & \\
\hline MONTE BELO & & 3 & 34 & $\bar{A}$ & 01/01/00 & RB85-5156 & $30 . \mathrm{C}$ & 0,3 & 6 & \begin{tabular}{|l|}
46 \\
\end{tabular} & 32 & & 0,05 & 0,25 & 0,23 & 0,23 & 0,00 & 5,42 & 9 & 79,07 & 99,99 \\
\hline MONTE BELO & & 3 & 34 & B & $01 / 01 / 00$ & RB85-5156 & $30 . \mathrm{C}$ & 0,4 & 3 & 39 & 20 & & 0,07 & 0,13 & 0,20 & 0,14 & 0,00 & 4,21 & & & \\
\hline MONTE BELO & & 3 & 35 & A & $01 / 01 / 00$ & RB85-5156 & $30 . \mathrm{C}$ & 0,6 & 4 & \begin{tabular}{|l|}
44 \\
\end{tabular} & 43 & & 0,10 & 0,17 & 0,22 & 1,05 & 0,00 & 7,39 & \begin{tabular}{|l|}
14,23 \\
\end{tabular} & 82,96 & 99,0 \\
\hline MONTE BELO & & 3 & 35 & $\mathrm{~B}$ & 01/01/00 & RB85-5156 & $30 . \mathrm{C}$ & 0,4 & 3 & \begin{tabular}{|l|}
34 \\
\end{tabular} & 47 & & 0,07 & 0,13 & 0,17 & 1,12 & 0,00 & 6,84 & & & \\
\hline MONTE BELO & & 3 & 36 & $\mathrm{~A}$ & 01/01/00 & RB85-5156 & $30 . C$ & 0,6 & 3 & 51 & \begin{tabular}{|l|}
17 \\
\end{tabular} & & 0,10 & 0,13 & 0,76 & 0,12 & 0,00 & 6,17 & \begin{tabular}{|l|}
11,87 \\
\end{tabular} & 80,96 & 71,87 \\
\hline MONTE BELO & & 3 & 36 & B & $01 / 01 / 00$ & RB85-5156 & $30 . \mathrm{C}$ & 0,4 & 3 & 56 & \begin{tabular}{|l|}
7 \\
\end{tabular} & & 0,07 & 0,13 & 0,81 & 0,05 & 0,00 & 5,70 & & & \\
\hline MONTE BELO & & 3 & 37 & $\mathrm{~A}$ & 01/01/00 & RB85-5156 & $30 . \mathrm{C}$ & 0,5 & 4 & \begin{tabular}{|l|}
48 \\
\end{tabular} & 20 & & 0,08 & $\mid 0,17$ & 0,24 & 0,14 & 0,00 & 5,12 & \begin{tabular}{|l|}
9,48 \\
\end{tabular} & 78,94 & 92,5 \\
\hline MONTE BELO & & 3 & 37 & B & $01 / 01 / 00$ & RB85-5156 & $30 . \mathrm{C}$ & 0,8 & 3 & \begin{tabular}{|l|}
42 \\
\end{tabular} & 19 & & 0,13 & 0,13 & 0,21 & 0,13 & 0,00 & 4,36 & & & \\
\hline SANTA FE & PV-1 & 1 & 2 & A & $01 / 01 / 00$ & RB85-5536 & $30 . \mathrm{C}$ & 1,3 & 4 & 35 & 54 & & 0,22 & 0,17 & 0,18 & 0,58 & 0,00 & \begin{tabular}{|l|}
6,19 \\
\end{tabular} & 13,68 & 82,50 & 88,38 \\
\hline SANTA FE & PV-2 & 1 & 2 & B & $01 / 01 / 00$ & RB85-5536 & $30 . \mathrm{C}$ & 1,0 & 3 & \begin{tabular}{|l|}
36 \\
\end{tabular} & 49 & & 0,17 & 0,13 & 0,18 & 1,15 & 0,00 & 7,49 & & & \\
\hline SANTA FE & PV-3 & 1 & 4 & A & $01 / 01 / 00$ & $\begin{array}{l}\text { RB85-5536 } \\
\end{array}$ & $30 . \mathrm{C}$ & 1,6 & 20 & \begin{tabular}{|l|}
58 \\
\end{tabular} & \begin{tabular}{|l|}
65 \\
\end{tabular} & & 0,27 & $\mid 0,63$ & 0,83 & 0,80 & 0,00 & 10,64 & 16,21 & 84,63 & 79,42 \\
\hline SANTA FE & $\mathrm{PV}-4$ & 1 & 4 & B & $01 / 01 / 00$ & RB85-5536 & $30 . \mathrm{C}$ & 0,7 & 4 & 77 & 32 & & 0,12 & 0,17 & 0,52 & 0,23 & 0,00 & 5,57 & & & \\
\hline SANTA FE & PV-5 & 1 & 6 & A & $01 / 01 / 00$ & RB85-5536 & $30 . \mathrm{C}$ & 1,8 & 21 & 98 & 86 & & 0,30 & 0,65 & 0,73 & 1,00 & 0,00 & 11,12 & \begin{tabular}{|l|}
20,65 \\
\end{tabular} & 88,39 & 62,35 \\
\hline SANTA FE & PV-6 & 1 & 6 & B & $01 / 01 / 00$ & RB85-5536 & $30 . \mathrm{C}$ & 1,2 & 4 & \begin{tabular}{|l|}
63 \\
\end{tabular} & 74 & & 0,20 & 0,17 & 0,88 & 0,98 & 0,00 & 9,53 & & & \\
\hline SANTA FE & PV-7 & 1 & 8 & A & $01 / 01 / 00$ & RB85-5536 & $30 . \mathrm{C}$ & 1,5 & 17 & \begin{tabular}{|l|}
54 \\
\end{tabular} & \begin{tabular}{|l|}
66 \\
\end{tabular} & & 0,25 & 0,55 & 0,79 & 0,82 & 0,00 & 10,38 & 20,41 & 88,18 & 78,70 \\
\hline SANTA FE & PV-8 & 1 & 8 & B & $01 / 01 / 00$ & RB85-5536 & $30 . \mathrm{C}$ & 1,3 & 16 & \begin{tabular}{|l|}
53 \\
\end{tabular} & \begin{tabular}{|l|}
66 \\
\end{tabular} & & 0,22 & 0,53 & 0,78 & 0,82 & 0,00 & 10,03 & & & \\
\hline SANTA FE & PV-9 & 1 & 9 & $\bar{A}$ & $01 / 01 / 00$ & $\begin{array}{l}\text { RB85-5536 } \\
\end{array}$ & $30 . \mathrm{C}$ & 2,1 & 58 & \begin{tabular}{|l|}
66 \\
\end{tabular} & \begin{tabular}{|l|}
77 \\
\end{tabular} & & 0,35 & 1,00 & 0,91 & 1,00 & 0,00 & 14,58 & 26,11 & 93,01 & 83,14 \\
\hline SANTA FE & $\mathrm{PV}-10$ & 1 & 9 & $\bar{B}$ & 01/01/00 & RB85-5536 & $30 . \mathrm{C}$ & 1,6 & 32 & 55 & \begin{tabular}{|l|}
70 \\
\end{tabular} & & 0,27 & 0,93 & 0,80 & 0,90 & 0,00 & 11,53 & & & \\
\hline SANTA FE & $\mathrm{PV}-11$ & 2 & 12 & A & $01 / 01 / 00$ & SP80-1842 & $30 . \mathrm{C}$ & 1,4 & 7 & 53 & 66 & & 0,23 & 0,28 & 0,78 & 0,82 & 0,00 & \begin{tabular}{|l|}
9,18 \\
\end{tabular} & \begin{tabular}{|l|}
14,83 \\
\end{tabular} & 83,47 & 41,40 \\
\hline SANTA FE & $\mathrm{PV}-12$ & 2 & 12 & $B$ & $01 / 01 / 00$ & SP80-1842 & $30 . \mathrm{C}$ & 0,6 & 2 & 43 & 58 & & 0,10 & 0,08 & 0,22 & 0,66 & 0,00 & 5,65 & & & \\
\hline SANTA FE & $\mathrm{PV}-13$ & 2 & 13 & A & $01 / 01 / 00$ & 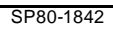 & $30 . \mathrm{C}$ & 2,2 & 6 & 79 & 60 & & 0,37 & 0,25 & 0,54 & 0,70 & 0,00 & 8,20 & 16,52 & 84,90 & 61,41 \\
\hline SANTA FE & PV-14 & 2 & 13 & B & $01 / 01 / 00$ & SP80-1842 & $30 . \mathrm{C}$ & 0,9 & 3 & \begin{tabular}{|l|}
91 \\
\end{tabular} & 72 & & 0,15 & 0,13 & 0,66 & 0,94 & 0,00 & 8,33 & & & \\
\hline SANTA FE & $\mathrm{PV}-15$ & 2 & 25 & $\bar{A}$ & $01 / 01 / 00$ & 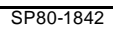 & $30 . \mathrm{C}$ & 1,1 & 9 & \begin{tabular}{|l|}
39 \\
\end{tabular} & 28 & & 0,18 & 0,33 & 0,20 & 0,20 & 0 & 5,43 & 12,43 & 81,43 & 74,6 \\
\hline SANTA FE & $\mathrm{PV}-16$ & 2 & 25 & B & $01 / 01 / 00$ & SP80-1842 & $30 . \mathrm{C}$ & 0,8 & 5 & \begin{tabular}{|l|}
46 \\
\end{tabular} & 39 & & 0,13 & 0,21 & 0,23 & 0,98 & 0 & \begin{tabular}{|l|}
6,99 \\
\end{tabular} & & & \\
\hline SANTANA & $\begin{array}{l}\text { LE2+LE3 - } \\
\text { LE3+PE }\end{array}$ & 27 & 52 & $\bar{A}$ & $01 / 01 / 00$ & RB83-5486 & $30 . \mathrm{C}$ & 4,5 & 37 & 46 & 56 & 50 & 0,75 & 1,00 & 0,23 & 0,62 & \begin{tabular}{|l|}
1,00 \\
\end{tabular} & 13,35 & 27,14 & 93,88 & 102,32 \\
\hline SANTANA & $\begin{array}{l}\mathrm{LE2}+\mathrm{LE} 3- \\
\mathrm{LE} 3+\mathrm{PE}\end{array}$ & 27 & 52 & $\bar{B}$ & $01 / 01 / 00$ & RB83-5486 & $30 . \mathrm{C}$ & 9,0 & 14 & 54 & 53 & \begin{tabular}{|l|}
63 \\
\end{tabular} & 1,00 & 0,47 & 0,79 & 0,56 & \begin{tabular}{|l|}
1,00 \\
\end{tabular} & 13,79 & & & \\
\hline \begin{tabular}{|l|} 
SANTANA \\
\end{tabular} & $\begin{array}{l}\text { LE2+LE3 - } \\
\text { LE3+PE }\end{array}$ & 27 & 54 & A & $01 / 01 / 00$ & RB83-5486 & $30 . \mathrm{C}$ & 8,1 & 88 & \begin{tabular}{|l|}
59 \\
\end{tabular} & \begin{tabular}{|l|}
62 \\
\end{tabular} & \begin{tabular}{|l|}
50 \\
\end{tabular} & 1,00 & 1,00 & 0,84 & 0,74 & 1,00 & 16,52 & 30,30 & 96,55 & 91,65 \\
\hline SANTANA & $\begin{array}{l}\text { LE2+LE3 - } \\
\text { LE3+PE }\end{array}$ & 27 & 54 & $\mathrm{~B}$ & 01/01/00 & $\begin{array}{l}\text { RB83-5486 } \\
\end{array}$ & $30 . \mathrm{C}$ & 9,3 & 13 & 54 & 53 & \begin{tabular}{|l|}
63 \\
\end{tabular} & 1,00 & 0,44 & 0,79 & 0,56 & 1,00 & 13,78 & & & \\
\hline SANTANA & $\begin{array}{l}\text { LE2+LE3 - } \\
\text { LE3+PE }\end{array}$ & 27 & 56 & A & $01 / 01 / 00$ & RB83-5486 & $30 . \mathrm{C}$ & 6,4 & 35 & 81 & 69 & \begin{tabular}{|l|}
50 \\
\end{tabular} & 1,00 & 1,00 & 0,56 & 0,88 & 1,00 & 16,55 & 27,50 & 94,18 & 95,32 \\
\hline \begin{tabular}{|l|} 
SANTANA \\
\end{tabular} & $\begin{array}{l}\text { LE2+LE3- } \\
\text { LE3+PE }\end{array}$ & 27 & 56 & $\bar{B}$ & $01 / 01 / 00$ & $\begin{array}{l}\text { RB83-5486 } \\
\end{array}$ & $30 . \mathrm{C}$ & 2,1 & 6 & 77 & 59 & \begin{tabular}{|l|}
63 \\
\end{tabular} & 0,35 & 0,25 & 0,52 & 0,68 & 1,00 & 10,95 & & & \\
\hline $\begin{array}{l}\text { SANTANA } \\
\end{array}$ & $\begin{array}{l}\text { LE2+LE3- } \\
\text { LE3+PE }\end{array}$ & 27 & 58 & A & $01 / 01 / 00$ & RB83-5486 & $30 . \mathrm{C}$ & 5,6 & 35 & 54 & 62 & \begin{tabular}{|l|}
50 \\
\end{tabular} & 0,93 & 1,00 & 0,79 & 0,74 & \begin{tabular}{|l|}
1,00 \\
\end{tabular} & 16,24 & 31,52 & 97,59 & 118,98 \\
\hline SANTANA & $\begin{array}{l}\text { LE2+LE3 - } \\
\text { LE3+PE }\end{array}$ & 27 & 58 & B & $01 / 01 / 00$ & RB83-5486 & $30 . \mathrm{C}$ & 7,1 & 6 & 65 & 47 & 63 & 1,00 & 0,25 & 0,90 & 1,12 & 1,00 & 15,28 & & & \\
\hline SANTANA & $\begin{array}{l}\text { LE2+LE3 - } \\
\text { LE3+PE }\end{array}$ & 27 & 59 & A & $01 / 01 / 00$ & RB83-5486 & $30 . \mathrm{C}$ & 8,3 & 17 & \begin{tabular}{|l|}
64 \\
\end{tabular} & 65 & \begin{tabular}{|l|}
50 \\
\end{tabular} & 1,00 & 0,55 & 0,89 & 0,80 & 1,00 & 15,65 & 28,61 & 95,12 & 89,86 \\
\hline SANTANA & $\begin{array}{l}\text { LE2+LE3 - } \\
\text { LE3+PE }\end{array}$ & 27 & 59 & $\bar{B}$ & $01 / 01 / 00$ & RB83-5486 & $30 . \mathrm{C}$ & 11,3 & 8 & 76 & 59 & \begin{tabular}{|l|}
63 \\
\end{tabular} & 1,00 & 0,31 & 0,51 & 0,68 & 1,00 & 12,96 & & & \\
\hline SAO JOSE & PV4 & 9 & 21 & $\bar{A}$ & $01 / 01 / 00$ & $\begin{array}{l}\mathrm{P} 81-3250 \\
\end{array}$ & $20 . \mathrm{C}$ & 8,9 & 119 & 158 & 84 & 13 & 1,00 & 1,00 & 1,00 & 1,00 & 0,27 & 18,81 & 34,37 & 100,00 & 87,8 \\
\hline SAO JOSE & PV4 & 9 & 21 & B & $01 / 01 / 00$ & SP81-3250 & $20 . \mathrm{C}$ & 4,4 & 32 & 111 & \begin{tabular}{|l|}
62 \\
\end{tabular} & 24 & 0,73 & 0,93 & 0,86 & 0,74 & 0,48 & 15,57 & & & \\
\hline SAO JOSE & PV4 & 9 & 22 & A & $01 / 01 / 00$ & RB84-5257 & $20 . \mathrm{C}$ & 2,6 & 24 & 62 & 24 & 13 & 0,43 & 0,73 & 0,87 & 0,17 & 0,27 & 11,38 & 24,47 & 91,62 & 89,93 \\
\hline SAO JOSE & $\mathrm{PV} 4$ & 9 & 22 & B & $01 / 01 / 00$ & RB84-5257 & $20 . \mathrm{C}$ & 1,6 & 19 & 55 & 38 & 24 & 0,27 & 0,60 & 0,80 & 0,97 & 0,48 & 13,09 & & & \\
\hline SAO LUIS & PV9 & 13 & 13 & A & $01 / 01 / 00$ & SP80-1842 & $30 . \mathrm{C}$ & 1,1 & 21 & 55 & 30 & 21 & 0,18 & 0,65 & 0,80 & 0,21 & 0,42 & \begin{tabular}{|l|}
9,73 \\
\end{tabular} & 22,04 & 89,57 & 101,09 \\
\hline SAO LUIS & PV9 & 13 & 13 & B & $01 / 01 / 00$ & SP80-1842 & $30 . \mathrm{C}$ & 1,3 & 10 & 54 & 37 & 43 & 0,22 & 0,36 & 0,79 & 0,95 & 0,86 & 12,31 & & & \\
\hline SAOLUIS & PV9 & 13 & 14 & A & $01 / 01 / 00$ & RB83-5486 & $20 . \mathrm{C}$ & 0,8 & 5 & 44 & 29 & 21 & 0,13 & 0,21 & 0,22 & 0,21 & 0,42 & 7,55 & 18,82 & 86,84 & 89,79 \\
\hline SAOLUIS & PV9 & 13 & 14 & $\mathrm{~B}$ & $01 / 01 / 00$ & $\begin{array}{l}\text { RB83-5486 } \\
\end{array}$ & $20 . \mathrm{C}$ & 1,6 & 5 & 58 & 27 & 43 & 0,27 & 0,21 & 0,83 & 0,19 & 0,86 & \begin{tabular}{|l|}
11,27 \\
\end{tabular} & & & \\
\hline SAO LUIS & PV9 & 13 & 19 & A & 01/01/00 & RB83-5486 & $30 . \mathrm{C}$ & 1,1 & 10 & 44 & \begin{tabular}{|l|}
22 \\
\end{tabular} & 21 & 0,18 & 0,36 & 0,22 & 0,16 & 0,42 & 6,95 & 15,97 & 84,43 & 80,75 \\
\hline SAO LUIS & PV9 & 13 & 19 & B & $01 / 01 / 00$ & B83-5486 & $30 . \mathrm{C}$ & 1,3 & 2 & 51 & 33 & 43 & 0,22 & 0,08 & 0,76 & 0,23 & 0,86 & \begin{tabular}{|l|}
9,01 \\
\end{tabular} & & & \\
\hline SAO LUIS & PV9 & 13 & 27 & A & $01 / 01 / 00$ & SP80-1842 & $30 . \mathrm{C}$ & 1,7 & 5 & 110 & 19 & 21 & 0,28 & 0,21 & 0,85 & 0,13 & 0,42 & 10,50 & 19,10 & 87,08 & 68,41 \\
\hline SAO LUIS & PV9 & 13 & 27 & B & $01 / 01 / 00$ & SP80-1842 & $30 . \mathrm{C}$ & 1,1 & 2 & 87 & 17 & 43 & 0,18 & 0,08 & 0,62 & 0,12 & 0,86 & 8,60 & & & \\
\hline OO LUIS & PV9 & 13 & 28 & $\bar{A}$ & $01 / 01 / 00$ & $\begin{array}{l}\text { RB83-5486 } \\
\end{array}$ & $30 . \mathrm{C}$ & 2,0 & 4 & 82 & 53 & 21 & 0,33 & 0,17 & 0,57 & 0,56 & 0,42 & 10,96 & 22,31 & 89,79 & 83,86 \\
\hline O LUIS & PV9 & 13 & 28 & $\bar{B}$ & 01/01/00 & RB83-5486 & $30 . \mathrm{C}$ & 1,1 & 8 & 83 & 69 & 43 & 0,18 & 0,31 & 0,58 & 0,88 & 0,86 & 11,35 & & & \\
\hline SAO LUIS & PV9 & 13 & 30 & A & $01 / 01 / 00$ & 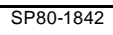 & $30 . \mathrm{C}$ & 1,8 & 2 & 95 & 15 & 21 & 0,30 & 0,08 & 0,70 & 0,11 & 0,42 & 7,76 & 15,94 & 84,41 & 81,91 \\
\hline SAO LUIS & PV9 & 13 & 30 & $B$ & 01/01/00 & SP80-1842 & $30 . \mathrm{C}$ & 1,1 & 2 & 79 & \begin{tabular}{|l|}
26 \\
\end{tabular} & 43 & 0,18 & 0,08 & 0,54 & 0,18 & 0,86 & 8,18 & & & \\
\hline SAO LUIS & PV9 & 7 & 70 & A & $01 / 01 / 00$ & SP80-1816 & $30 . \mathrm{C}$ & 8,1 & 6 & 71 & 64 & 21 & 1,00 & 0,25 & 0,96 & 0,78 & 0,42 & 15,04 & 30,13 & 96,41 & 94,60 \\
\hline OLUIS & PV9 & 7 & 70 & B & $01 / 01 / 00$ & SP80-1816 & $30 . \mathrm{C}$ & 7,0 & 2 & 71 & 46 & 43 & 1,00 & 0,08 & 0,96 & 1,10 & 0,86 & 15,09 & & & \\
\hline OLUIS & PV9 & 6 & 77 & $\bar{A}$ & 01/01/00 & RB85-5453 & $30 . \mathrm{C}$ & 10,0 & 98 & 1114 & 72 & 21 & 1,00 & 1,0 & 0,89 & 0,94 & \begin{tabular}{|l|}
0,42 \\
\end{tabular} & 17,56 & 32,96 & 98,81 & $\overline{02,4}$ \\
\hline
\end{tabular}




\begin{tabular}{|c|c|c|c|c|c|c|c|c|c|c|c|c|c|c|c|c|c|c|c|c|c|}
\hline nome & & & & & & & & teores & & & & & pontuação & & & & & & & & \\
\hline Fazenda & Solo & zona & talhão & horiz & coleta & $\begin{array}{l}\text { Variedade } \\
\end{array}$ & Estágio & $\mathrm{K}$ & $P$ & \begin{tabular}{|l|l|}
$T$ \\
\end{tabular} & $\mathrm{~V}$ & Arg & $\mathrm{K}$ & $P$ & $\bar{T}$ & $\mathrm{~V}$ & Arg & $\overline{\mathrm{NP}}$ & \begin{tabular}{|l|} 
Ps \\
\end{tabular} & prod estima & \begin{tabular}{|l|} 
prod medida \\
\end{tabular} \\
\hline SAO LUIS & PV9 & $\overline{6}$ & 77 & $\bar{B}$ & $01 / 01 / 00$ & RB85-5453 & 30.C & $\overline{9,2}$ & 8 & 86 & 63 & 43 & 1,00 & 0,31 & 0,61 & 0,76 & 0,86 & 15,41 & & & \\
\hline$\overline{A O L U I S}$ & PV9 & 6 & 78 & $\bar{A}$ & $01 / 01 / 00$ & BB85-5453 & 30.C & 6,1 & 15 & 87 & 51 & 21 & 1,00 & 0,50 & 0,62 & 0,52 & 0,42 & 12,19 & 25,00 & 92,07 & 96,8 \\
\hline SAO LUIS & PV9 & 6 & 78 & B & 01/01/00 & RB85-5453 & $30 . C$ & 4,9 & 3 & 80 & 35 & 43 & 0,82 & 0,13 & 0,55 & 0,92 & 0,86 & 12,81 & & & \\
\hline SAO LUIS & PV9 & 6 & 80 & A & $01 / 01 / 00$ & RB85-5453 & $30 . \mathrm{C}$ & 5,2 & 105 & 108 & 40 & 21 & 0,87 & 1,00 & 0,83 & 1,00 & 0,42 & 17,16 & 31,68 & 97,73 & 82,9 \\
\hline SAO LUIS & PV9 & 6 & 80 & B & $01 / 01 / 00$ & RB85-5453 & $30 . \mathrm{C}$ & 2,7 & 24 & 88 & 46 & \begin{tabular}{|l|}
43 \\
\end{tabular} & 0,45 & 0,73 & 0,63 & 1,10 & 0,86 & 14,52 & & & \\
\hline$\overline{\text { SAOLUIS }}$ & PV9 & 6 & 82 & $\bar{A}$ & $01 / 01 / 00$ & 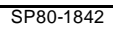 & $30 . \mathrm{C}$ & 6,5 & 28 & 82 & 48 & \begin{tabular}{|l|}
21 \\
\end{tabular} & 1,00 & 0,83 & \begin{tabular}{|l|}
0,57 \\
\end{tabular} & 1,13 & 0,42 & $\mid 16,66$ & 26,90 & 93,67 & 115,92 \\
\hline SAO LUIS & PV9 & 6 & 82 & $\bar{B}$ & $01 / 01 / 00$ & 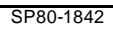 & $30 . \mathrm{C}$ & 1,1 & 5 & \begin{tabular}{|l|}
67 \\
\end{tabular} & 34 & \begin{tabular}{|l|}
43 \\
\end{tabular} & 0,18 & 0,21 & 0,92 & 0,24 & 0,86 & 10,24 & & & \\
\hline SAO LUIS & PV9 & 6 & 83 & $\mathrm{~A}$ & $01 / 01 / 00$ & $\begin{array}{l}\text { SP80-1842 } \\
\text { (1) }\end{array}$ & $30 . \mathrm{C}$ & 8,4 & 34 & 93 & 69 & 21 & 1,00 & 0,98 & 0,68 & 0,88 & 0,42 & 16,67 & 32,33 & 98,27 & 82,1 \\
\hline SAOLUIS & PV9 & 6 & 83 & B & 01/01/00 & SP80-1842 & $30 . \mathrm{C}$ & 8,4 & 35 & 86 & 60 & \begin{tabular}{|l|}
43 \\
\end{tabular} & 1,00 & 1,00 & \begin{tabular}{|l|}
0,61 \\
\end{tabular} & 0,70 & 0,86 & $\mid 15,66$ & & & \\
\hline SAOLUIS & PV9 & 6 & 84 & A & $01 / 01 / 00$ & SP80-1842 & $30 . \mathrm{C}$ & 6,7 & 21 & 85 & \begin{tabular}{|l|}
57 \\
\end{tabular} & 21 & 1,00 & $\mid 0,65$ & $|0|$ & 0,64 & 0,42 & 14,74 & 29,24 & 95,66 & $\overline{106,6}$ \\
\hline SAO LUIS & PV9 & 6 & 84 & B & $01 / 01 / 00$ & SP80-1842 & $30 . \mathrm{C}$ & 5,7 & 15 & 71 & 52 & \begin{tabular}{|l|}
43 \\
\end{tabular} & 0,95 & 0,50 & 0,96 & 0,54 & 0,86 & 14,51 & & & \\
\hline SAO LUIS & PV9 & 6 & 85 & A & $01 / 01 / 00$ & SP80-1842 & $30 . \mathrm{C}$ & 7,6 & 30 & 89 & 57 & 21 & 1,00 & 0,88 & 0,64 & 0,64 & 0,42 & 15,53 & 29,57 & 95,94 & 108,9 \\
\hline SAOLUIS & PV9 & 6 & 85 & $\bar{B}$ & $01 / 01 / 00$ & 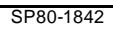 & $30 . \mathrm{C}$ & 6,9 & 7 & 75 & 41 & \begin{tabular}{|l|}
43 \\
\end{tabular} & 1,00 & 0,28 & 0,50 & 1,02 & 0,86 & 14,04 & & & \\
\hline SAO LUIS & PV9 & 6 & 87 & A & $01 / 01 / 00$ & 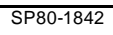 & $30 . \mathrm{C}$ & 4,4 & 30 & \begin{tabular}{|l|}
92 \\
\end{tabular} & 54 & \begin{tabular}{|l|}
21 \\
\end{tabular} & 0,73 & 0,88 & \begin{tabular}{|l|}
0,67 \\
\end{tabular} & 0,58 & 0,42 & $\mid 14,64$ & 27,59 & 94,26 & 105,7 \\
\hline SAO LUIS & PV9 & 6 & 87 & B & $01 / 01 / 00$ & SP80-1842 & $30 . \mathrm{C}$ & 3,5 & 8 & \begin{tabular}{|l|}
55 \\
\end{tabular} & \begin{tabular}{|l|}
62 \\
\end{tabular} & \begin{tabular}{|l|}
43 \\
\end{tabular} & 0,58 & 0,31 & \begin{tabular}{|l|}
0,80 \\
\end{tabular} & 0,74 & 0,86 & 12,94 & & & \\
\hline SAO LUIS & PV9 & 6 & 88 & A & $01 / 01 / 00$ & SP80-1842 & $30 . \mathrm{C}$ & 6,0 & 6 & 85 & 37 & 21 & 1,00 & 0,25 & \begin{tabular}{|l|}
0,60 \\
\end{tabular} & 0,95 & 0,42 & 14,47 & 28,33 & 94,88 & 89,0 \\
\hline SAO LUIS & PV9 & 6 & 88 & B & $01 / 01 / 00$ & SP80-1842 & $30 . \mathrm{C}$ & 9,9 & 5 & 71 & 52 & 43 & 1,00 & 0,21 & 0,96 & 0,54 & 0,86 & 13,86 & & & \\
\hline SAO LUIS & PV9 & 6 & 89 & A & $01 / 01 / 00$ & SP80-1842 & $30 . \mathrm{C}$ & 2,4 & 3 & 68 & 38 & 21 & 0,40 & 0,13 & 0,93 & 0,97 & 0,42 & 11,31 & 20,05 & 87,88 & $117,9 !$ \\
\hline SAO LUIS & PV9 & 6 & 89 & $\bar{B}$ & $01 / 01 / 00$ & 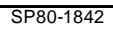 & $30 . \mathrm{C}$ & 1,2 & 1 & \begin{tabular}{|l|}
92 \\
\end{tabular} & 13 & \begin{tabular}{|l|l|}
43 \\
\end{tabular} & 0,20 & 0,04 & \begin{tabular}{|l|l|}
0,67 \\
\end{tabular} & 0,09 & 0,86 & \begin{tabular}{|l|}
8,74 \\
\end{tabular} & & & \\
\hline SAO LUIS & PV9 & 6 & 90 & $\mathrm{~A}$ & $01 / 01 / 00$ & $\begin{array}{l}\text { SP80-1842 } \\
\text {. }\end{array}$ & $30 . \mathrm{C}$ & 1,2 & \begin{tabular}{|l|}
19 \\
\end{tabular} & 51 & 60 & 21 & 0,20 & 0,60 & 0,76 & 0,70 & 0,42 & 10,75 & 20,70 & 88,43 & 97,7 \\
\hline SAOLUIS & PV9 & 6 & 90 & B & 01/01/00 & 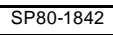 & 30.C & 1,0 & 2 & 41 & \begin{tabular}{|l|}
46 \\
\end{tabular} & \begin{tabular}{|l|l|}
43 \\
\end{tabular} & 0,17 & 0,08 & 0,21 & 1,10 & 0,86 & \begin{tabular}{|l|}
9,95 \\
\end{tabular} & & & \\
\hline SAO LUIS & PV9 & 6 & 91 & A & $01 / 01 / 00$ & SP80-1842 & $30 . \mathrm{C}$ & 4,6 & 15 & 81 & 48 & \begin{tabular}{|l|}
21 \\
\end{tabular} & 0,77 & 0,50 & 0,56 & 1,13 & 0,42 & $\mid 14,95$ & 28,57 & 95,09 & $\overline{120,6}$ \\
\hline SAO LUIS & PV9 & 6 & 91 & B & $01 / 01 / 00$ & SP80-1842 & $30 . \mathrm{C}$ & 7,1 & 5 & 70 & 51 & 43 & 1,00 & 0,21 & 0,95 & 0,52 & 0,86 & 13,62 & & & \\
\hline SAO LUIS & PV9 & 6 & 92 & A & $01 / 01 / 00$ & SP80-1842 & $30 . \mathrm{C}$ & 7,0 & 9 & 89 & 47 & 21 & 1,00 & 0,33 & 0,64 & 1,12 & 0,42 & 15,34 & 31,09 & 97,23 & 92,38 \\
\hline SAO LUIS & PV9 & 6 & 92 & B & $01 / 01 / 00$ & $\begin{array}{l}\text { SP80-1842 } \\
\end{array}$ & $30 . \mathrm{C}$ & 4,7 & 2 & 69 & 39 & 43 & 0,78 & 0,08 & 0,94 & 0,98 & 0,86 & 15,75 & & & \\
\hline SAO LUIS & PV9 & 6 & 93 & A & $01 / 01 / 00$ & 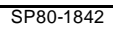 & $30 . \mathrm{C}$ & 9,1 & 8 & 83 & 54 & \begin{tabular}{|l|}
21 \\
\end{tabular} & 1,00 & 0,31 & 0,58 & 0,58 & 0,42 & 13,46 & 26,10 & 93,00 & 128,0 \\
\hline SAOLUIS & PV9 & 6 & 93 & B & $01 / 01 / 00$ & 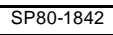 & $30 . \mathrm{C}$ & 7,7 & 4 & 76 & 55 & \begin{tabular}{|l|l|}
43 \\
\end{tabular} & 1,00 & 0,17 & 0,51 & 0,60 & 0,86 & 12,64 & & & \\
\hline SAO LUIS & PV9 & 8008 & 131 & A & $01 / 01 / 00$ & RB72-454 & $40 . \mathrm{C}$ & 4,9 & 7 & 124 & 58 & \begin{tabular}{|l|}
21 \\
\end{tabular} & 0,82 & 0,28 & 0,99 & 0,66 & 0,42 & 11,75 & 19,88 & 87,74 & 110,0 \\
\hline SAO LUIS & PV9 & 8008 & 131 & B & $01 / 01 / 00$ & RB72-454 & $40 . \mathrm{C}$ & 2,6 & 2 & 77 & 16 & 43 & 0,43 & 0,08 & 0,52 & 0,11 & 0,86 & 8,13 & & & \\
\hline SAO LUIS & PV9 & 8008 & 132 & $\bar{A}$ & $01 / 01 / 00$ & RB72-454 & $40 . \mathrm{C}$ & 2,8 & 3 & \begin{tabular}{|l|}
63 \\
\end{tabular} & 50 & \begin{tabular}{|l|}
21 \\
\end{tabular} & 0,47 & 0,13 & \begin{tabular}{|c|}
0,88 \\
\end{tabular} & 0,50 & 0,42 & \begin{tabular}{|l|}
9,06 \\
\end{tabular} & 20,13 & 87,95 & 100,3 \\
\hline SAO LUIS & PV9 & 8008 & 132 & B & $01 / 01 / 00$ & RB72-454 & $40 . C$ & 3,3 & 4 & 69 & 50 & \begin{tabular}{|l|}
43 \\
\end{tabular} & 0,55 & 0,17 & 0,94 & 0,50 & 0,86 & 11,08 & & & \\
\hline SAO LUIS & PV9 & 9009 & 149 & $\mathrm{~A}$ & $01 / 01 / 00$ & RB83-5054 & $40 . \mathrm{C}$ & 4,1 & 47 & 72 & 47 & 21 & 0,68 & 1,00 & 0,97 & 1,12 & 0,42 & $\mid 16,41$ & 27,46 & 94,15 & 107,16 \\
\hline SAO LUIS & PV9 & 9009 & 149 & $\bar{B}$ & $01 / 01 / 00$ & $\begin{array}{l}\text { RB83-5054 } \\
\end{array}$ & $40 . \mathrm{C}$ & 2,8 & 17 & 66 & 28 & \begin{tabular}{|l|}
43 \\
\end{tabular} & 0,47 & 0,55 & 0,91 & 0,20 & 0,86 & 111,06 & & & \\
\hline SAO LUIS & PV9 & 9009 & 150 & A & $01 / 01 / 00$ & RB83-5054 & $40 . \mathrm{C}$ & 3,5 & 5 & 81 & 20 & 21 & 0,58 & 0,21 & 0,56 & 0,14 & 0,42 & \begin{tabular}{|l|}
7,92 \\
\end{tabular} & 17,01 & 85,31 & 106,81 \\
\hline SAO LUIS & PV9 & 9009 & 150 & B & $01 / 01 / 00$ & RB83-5054 & $40 . \mathrm{C}$ & 2,8 & 3 & 58 & 10 & 43 & 0,47 & 0,13 & 0,83 & 0,07 & 0,86 & 9,08 & & & \\
\hline SAO LUIS & PV9 & 9009 & 151 & A & $01 / 01 / 00$ & RB83-5054 & $40 . \mathrm{C}$ & 2,6 & 6 & 93 & 13 & 21 & 0,43 & 0,25 & 0,68 & 0,09 & 0,42 & \begin{tabular}{|l|}
9,46 \\
\end{tabular} & 17,69 & 85,88 & $\overline{86,3}$ \\
\hline SAO LUIS & PV9 & 9009 & 151 & B & 01/01/00 & RB83-5054 & $40 . \mathrm{C}$ & 1,1 & 3 & 67 & 4 & \begin{tabular}{|l|}
43 \\
\end{tabular} & 0,18 & 0,13 & 0,92 & 0,03 & 0,86 & 8,23 & & & \\
\hline SAO LUIS & PV9 & 9009 & 152 & A & 01/01/00 & RB83-5054 & $40 . \mathrm{C}$ & 2,0 & 6 & 79 & 8 & \begin{tabular}{|l|}
21 \\
\end{tabular} & 0,33 & 0,25 & 0,54 & 0,06 & 0,42 & 7,06 & 15,86 & 84,34 & 87,6 \\
\hline SAOLUIS & PV9 & 9009 & 152 & B & $01 / 01 / 00$ & RB83-5054 & $40 . C$ & 1,6 & 4 & \begin{tabular}{|l|}
68 \\
\end{tabular} & \begin{tabular}{|l|l|}
5 \\
\end{tabular} & 43 & 0,27 & 0,17 & \begin{tabular}{|l|}
0,93 \\
\end{tabular} & 0,04 & 0,86 & \begin{tabular}{|l|}
8,80 \\
\end{tabular} & & & \\
\hline SAO LUIS & PV9 & 9009 & 153 & A & $01 / 01 / 00$ & RB83-5054 & $40 . \mathrm{C}$ & 4,0 & 6 & 60 & 53 & 21 & 0,67 & 0,25 & 0,85 & 0,56 & 0,42 & 10,35 & 19,34 & 87,28 & 94,78 \\
\hline SAO LUIS & PV9 & 9009 & 153 & B & $01 / 01 / 00$ & RB83-5054 & $40 . \mathrm{C}$ & 2,6 & 3 & 47 & 61 & \begin{tabular}{|l|}
43 \\
\end{tabular} & 0,43 & 0,13 & 0,24 & 0,72 & 0,86 & \begin{tabular}{|l|}
9,00 \\
\end{tabular} & & & \\
\hline SAO LUIS & PV9 & 9009 & 154 & A & $01 / 01 / 00$ & RB83-5054 & $40 . C$ & 3,1 & 4 & 63 & 25 & 21 & 0,52 & 0,17 & 0,88 & 0,18 & 0,42 & \begin{tabular}{|l|}
8,59 \\
\end{tabular} & 14,73 & 83,38 & 97,0 \\
\hline SAO LUIS & PV9 & 9009 & 154 & B & $01 / 01 / 00$ & RB83-5054 & $40 . \mathrm{C}$ & 1,5 & 3 & 46 & 8 & \begin{tabular}{|l|}
43 \\
\end{tabular} & 0,25 & 0,13 & 0,23 & 0,06 & 0,86 & \begin{tabular}{|l|}
6,14 \\
\end{tabular} & & & \\
\hline SAO LUIS & PV9 & 9009 & 155 & A & | & RB83-5054 & $40 . C$ & 2,2 & 13 & 72 & 27 & \begin{tabular}{|l|}
21 \\
\end{tabular} & 0,37 & 0,44 & \begin{tabular}{|l|}
0,97 \\
\end{tabular} & 0,19 & 0,42 & \begin{tabular}{|l|}
9,06 \\
\end{tabular} & 16,47 & 84,85 & 82,80 \\
\hline SAO LUIS & PV9 & 9009 & 155 & B & $01 / 0$ & $\begin{array}{l}\text { RB83-5054 } \\
\end{array}$ & $40 . \mathrm{C}$ & 1,0 & 3 & 50 & \begin{tabular}{|l|}
6 \\
\end{tabular} & \begin{tabular}{|l|}
43 \\
\end{tabular} & 0,17 & 0,13 & 0,75 & 0,04 & 0,86 & 7,41 & & & \\
\hline SAO LUIS & PV9 & 9009 & 156 & A & $01 / 01 / 00$ & RB83-5054 & $40 . \mathrm{C}$ & 1,3 & 6 & 68 & 23 & 21 & 0,22 & 0,25 & 0,93 & 0,16 & 0,42 & \begin{tabular}{|l|}
7,82 \\
\end{tabular} & 14,31 & 83,02 & 86,9 \\
\hline SAO LUIS & PV9 & 9009 & 156 & B & $01 / 01 / 00$ & RB83-5054 & $40 . \mathrm{C}$ & 0,9 & 3 & 47 & 34 & 43 & 0,15 & 0,13 & 0,24 & 0,24 & 0,86 & 6,48 & & & \\
\hline SAO ROQUE & PV11 & 9 & 20 & A & | & RB82-5336 & $30 . \mathrm{C}$ & 2,9 & 12 & 62 & 16 & & 0,48 & 0,42 & $\mid 0,87$ & 0,11 & 0 & 8,28 & 22,62 & 90,06 & 108,95 \\
\hline SAO ROQUE & PV11 & 9 & 20 & $\bar{B}$ & $01 / 01 / 00$ & $\begin{array}{l}\text { RB82-5336 } \\
\end{array}$ & $30 . \mathrm{C}$ & 6,2 & 28 & 68 & 49 & & 1,00 & 0,83 & 0,93 & 1,15 & 0,00 & 14,34 & & & \\
\hline \begin{tabular}{|l|} 
STA.TER. \\
\end{tabular} & PE & 14 & 4 & A & $01 / 01 / 00$ & RB85-5156 & $20 . \mathrm{C}$ & \begin{tabular}{|l|l|}
1,7 \\
\end{tabular} & 15 & 39 & 28 & 42 & 0,28 & 0,50 & 0,20 & 0,20 & 0,84 & \begin{tabular}{|l|}
9,95 \\
\end{tabular} & 19,37 & 87,30 & 82,0 \\
\hline STA.TER.1 & $\mathrm{PE}$ & 14 & 4 & B & $01 / 01 / 00$ & RB85-5156 & $20 . \mathrm{C}$ & 2,0 & 6 & 32 & 31 & \begin{tabular}{|l|}
64 \\
\end{tabular} & 0,33 & 0,25 & 0,16 & 0,22 & 1,00 & \begin{tabular}{|l|}
9,42 \\
\end{tabular} & & & \\
\hline STA.TER. 1 & PE & 14 & 6 & A & $01 / 01 / 00$ & RB85-5113 & $20 . \mathrm{C}$ & 1,6 & 45 & 58 & 62 & 42 & 0,27 & 1,00 & 0,83 & 0,74 & 0,84 & 15,08 & 26,51 & 93,34 & 102,85 \\
\hline STA.TER. I & $\mathrm{PE}$ & 14 & 6 & B & $01 / 01 / 00$ & RB85-5113 & $20 . C$ & 0,8 & 3 & 54 & 53 & 64 & 0,13 & 0,13 & 0,79 & 0,56 & 1,00 & $\mid 11,43$ & & & \\
\hline AREIA BRAN & $\overline{\text { STAB }}$ & 7 & 77 & A & $01 / 02 / 00$ & SP80-1816 & $20 . \mathrm{C}$ & 0,6 & 4 & 55 & 23 & & 0,10 & 0,17 & 0,80 & 0,16 & 0,00 & \begin{tabular}{|l|}
7,97 \\
\end{tabular} & 14,97 & 83,58 & 67,58 \\
\hline AREIA BRAN & STA B & 7 & 77 & $\bar{B}$ & $01 / 02 / 00$ & SP80-1816 & $20 . \mathrm{C}$ & 0,3 & 1 & 60 & 13 & & 0,05 & 0,04 & 0,85 & 0,09 & 0,00 & 7,00 & & & \\
\hline PORT FELIZ & STA B & 2 & 22 & A & $01 / 02 / 00$ & RB85-5113 & $30 . \mathrm{C}$ & 0,8 & 4 & 65 & 52 & & 0,13 & 0,17 & 0,90 & 0,54 & 0,00 & \begin{tabular}{|l|}
8,07 \\
\end{tabular} & 15,38 & 83,93 & 83,39 \\
\hline PORT FELIZ & STA B & 2 & 22 & B & 01/02/00 & RB85-5113 & $30 . \mathrm{C}$ & 0,6 & 1 & 70 & 51 & & 0,10 & 0,04 & 0,95 & 0,52 & 0,00 & 7,31 & & & \\
\hline MIRANTE & & 42 & 6 & A & $05 / 00$ & 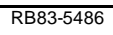 & $30 . \mathrm{C}$ & 2,0 & 43 & 81 & 38 & & 0,33 & 1,00 & 0,56 & $\mid 0,97$ & 0,00 & $\mid 11,06$ & 19,50 & 87,42 & 98,9 \\
\hline MIRANTE & & 42 & 6 & B & $23 / 05 / 00$ & $\begin{array}{l}\text { RB83-5486 } \\
\end{array}$ & $30 . \mathrm{C}$ & \begin{tabular}{|l|}
1,0 \\
\end{tabular} & 9 & 70 & 52 & & 0,17 & 0,33 & 0,95 & 0,54 & 0,00 & 8,45 & & & \\
\hline MIRANTE & & 42 & 7 & A & $23 / 05 / 00$ & $\begin{array}{l}\text { RB83-5486 } \\
\end{array}$ & $30 . \mathrm{C}$ & \begin{tabular}{|l|}
2,0 \\
\end{tabular} & 43 & 81 & 38 & & 0,33 & 1,00 & 0,56 & 0,97 & 0,00 & \begin{tabular}{|l|}
11,06 \\
\end{tabular} & 19,50 & 87,42 & 44,33 \\
\hline IRANTE & & 42 & 7 & B & \begin{tabular}{|l|}
$23 / 05 / 00$ \\
\end{tabular} & RB83-5486 & $30 . C$ & 1,0 & 9 & 70 & 52 & & 0,17 & 0,33 & 0,95 & 0,54 & 0,00 & 8 & & & \\
\hline$\overline{\mathrm{AQUA}}$ & $\begin{array}{l}\text { STA B } \\
\end{array}$ & 34 & 82 & A & 00 & RB83-5486 & $30 . \mathrm{C}$ & 0,5 & 5 & 52 & 40 & & 0,08 & 0,21 & 0,77 & 1,00 & 0,00 & \begin{tabular}{|l|}
8,66 \\
\end{tabular} & 15,69 & 84,19 & 62,98 \\
\hline AQUA & STA B & 34 & 82 & B & $20 / 06 / 00$ & RB83-5486 & $30 . \mathrm{C}$ & 0,5 & 3 & 48 & 41 & & 0,08 & 0,13 & 0,24 & 1,02 & 0,00 & 7,02 & & & \\
\hline ARAQUA & STAB & 34 & 84 & $\bar{A}$ & \begin{tabular}{|l|}
$20 / 06 / 00$ \\
\end{tabular} & 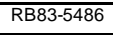 & $30 . \mathrm{C}$ & 0,6 & 5 & 39 & 58 & & 0,10 & 0,21 & 0,20 & 0,66 & 0,00 & \begin{tabular}{|l|}
5,97 \\
\end{tabular} & \begin{tabular}{|l|}
9,64 \\
\end{tabular} & 79,07 & 61,01 \\
\hline ARAQUA & STA B & 34 & 84 & B & $20 / 06 / 00$ & $\begin{array}{l}\text { RB83-5486 } \\
\end{array}$ & $30 . \mathrm{C}$ & 0,5 & 1 & 38 & 26 & & 0,08 & 0,04 & 0,19 & 0,18 & 0,00 & \begin{tabular}{|l|}
3,67 \\
\end{tabular} & & & \\
\hline LEITE I & & 11 & 31 & A & |05/07/00 & SP80-1842 & 30.C & 0,6 & 4 & \begin{tabular}{|l|}
42 \\
\end{tabular} & 57 & & 0,10 & 0,17 & 0,21 & 0,64 & 0,00 & \begin{tabular}{|l|}
6,50 \\
\end{tabular} & 10,68 & 79,95 & 66,86 \\
\hline LEITE I & & 11 & 31 & B & $05 / 07 / 00$ & SP80-1842 & $30 . \mathrm{C}$ & 0,1 & 1 & 30 & 33 & & 0,02 & 0,04 & 0,15 & 0,23 & 0 & 4,18 & & & \\
\hline LEITE I & & 11 & 34 & A & 05/07/00 & RB85-5156 & $40 . \mathrm{C}$ & 0,5 & 1 & 42 & 52 & & 0,08 & 0,04 & 0,21 & 0,54 & 0,00 & 4,80 & 8,05 & 77,73 & 48,4 \\
\hline
\end{tabular}


84

\begin{tabular}{|c|c|c|c|c|c|c|c|c|c|c|c|c|c|c|c|c|c|c|c|c|c|}
\hline nome & & & & & & & & teores & & & & & pontuação & & & & & & & & \\
\hline Fazenda & Solo & zona & talhão & horiz & coleta & Variedade & Estágio & $\mathrm{K}$ & $P$ & \begin{tabular}{|l}
$T$ \\
\end{tabular} & v & Arg & $\mathrm{K}$ & $P$ & \begin{tabular}{|l|l|}
$T$ \\
\end{tabular} & $\mathrm{v}$ & \begin{tabular}{|l|} 
Arg \\
\end{tabular} & $\mathrm{NP}$ & Ps & od estima & prod medid: \\
\hline $\mid$\begin{tabular}{|l|} 
P.LEITE I \\
\end{tabular} & & 11 & 34 & $\bar{B}$ & $05 / 07 / 00$ & RB85-5156 & $40 . \mathrm{C}$ & 0,1 & 1 & 33 & 33 & & 0,02 & 0,04 & 0,17 & 0,23 & 0,00 & 3,25 & & & \\
\hline LEITE I & & 11 & 35 & A & $05 / 07 / 00$ & RB85-5156 & $40 . \mathrm{C}$ & 1,0 & 3 & \begin{tabular}{|l|}
50 \\
\end{tabular} & 50 & & 0,17 & 0,13 & \begin{tabular}{|c|}
0,75 \\
\end{tabular} & 0,50 & $0,0,0$ & 8,45 & 11,86 & 80,95 & 54,35 \\
\hline P.LEITE I & & 11 & 35 & B & $05 / 07 / 00$ & RB85-5156 & $40 . \mathrm{C}$ & 0,3 & 1 & \begin{tabular}{|l|}
42 \\
\end{tabular} & \begin{tabular}{|l|}
19 \\
\end{tabular} & & 0,05 & $\mid 0,04$ & 0,21 & 0,13 & 0,0 & 3,41 & & & \\
\hline \begin{tabular}{|l} 
PINHEIR IV \\
\end{tabular} & & 12 & 2 & A & $05 / 07 / 00$ & SP80-1842 & $40 . \mathrm{C}$ & 0,5 & 3 & \begin{tabular}{|l|}
48 \\
\end{tabular} & 41 & & 0,08 & 0,13 & 0,24 & 1,02 & 0,00 & 8,22 & 11,12 & 80,33 & \begin{tabular}{ll|}
46,90 \\
\end{tabular} \\
\hline PINHEIR IV & & 12 & 2 & B & $05 / 07 / 00$ & SP80-1842 & $40 . \mathrm{C}$ & 0,3 & 1 & \begin{tabular}{|l|}
37 \\
\end{tabular} & $\mid 16$ & & 0,05 & 0,04 & 0,19 & 0,11 & 0,00 & 2,90 & & & \\
\hline PINHEIR.II & & 21 & 36 & A & \begin{tabular}{|l|}
$11 / 07 / 00$ \\
\end{tabular} & RB72-454 & $40 . \mathrm{C}$ & 0,5 & 3 & \begin{tabular}{|l|}
40 \\
\end{tabular} & 50 & & 0,08 & 0,13 & 0,20 & 0,50 & 0,00 & 6,55 & 14,57 & 83,25 & 76,16 \\
\hline PINHEIR.II & & 21 & 36 & B & $11 / 07 / 00$ & RB72-454 & $40 . \mathrm{C}$ & $\overline{0,3}$ & 3 & \begin{tabular}{|l|}
38 \\
\end{tabular} & \begin{tabular}{|l|}
42 \\
\end{tabular} & & 0,05 & 0,13 & 0,19 & 1,03 & 0,00 & 8,02 & & & \\
\hline \begin{tabular}{|l|} 
PINHEIR.II \\
\end{tabular} & & 21 & 42 & A & \begin{tabular}{|l|}
$11 / 07 / 00$ \\
\end{tabular} & RB72-454 & $40 . \mathrm{C}$ & 0,8 & 10 & 55 & \begin{tabular}{|l|}
49 \\
\end{tabular} & & 0,13 & 0,36 & 0,80 & 1,15 & 0,00 & 11,16 & 17,90 & 86,06 & 82,13 \\
\hline PINHEIR.II & & 21 & 42 & B & $111 / 07 / 00$ & RB72-454 & $40 . \mathrm{C}$ & 0,6 & 5 & \begin{tabular}{|l|}
47 \\
\end{tabular} & 40 & & 0,10 & 0,21 & \begin{tabular}{|l|}
0,24 \\
\end{tabular} & 1,00 & 0,00 & 6,73 & & & \\
\hline PINHEIR.II & & 21 & 43 & $\mathrm{~A}$ & \begin{tabular}{|l|}
$11 / 07 / 00$ \\
\end{tabular} & RB72-454 & $40 . \mathrm{C}$ & 1,0 & 4 & \begin{tabular}{|l|}
44 \\
\end{tabular} & \begin{tabular}{|l|}
43 \\
\end{tabular} & & 0,17 & 0,17 & 0,22 & 1,05 & 0,00 & 8,64 & 15,08 & 83,68 & 77,54 \\
\hline PINHEIR.II & & 21 & 43 & B & $11 / 07 / 00$ & RB72-454 & $40 . \mathrm{C}$ & 0,5 & 3 & 41 & 39 & & 0,08 & 0,13 & 0,21 & \begin{tabular}{|l|}
0,98 \\
\end{tabular} & 0,00 & 6,44 & & & \\
\hline PINHEIR.II & & 23 & 73 & 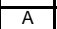 & 11/07/00 & RB80-6043 & $50 . \mathrm{C}$ & 1,0 & 4 & 33 & 60 & & 0,17 & $\mid 0,17$ & 0,17 & 0,70 & 0,00 & 6,45 & 11,21 & 80,41 & 54,5 \\
\hline PINHEIR.II & & 23 & 73 & B & \begin{tabular}{|l|}
$11 / 07 / 00$ \\
\end{tabular} & RB80-6043 & $50 . \mathrm{C}$ & 0,3 & 1 & 31 & 41 & & 0,05 & 0,04 & 0,16 & 1,02 & 0,00 & 4,77 & & & \\
\hline \begin{tabular}{|l|} 
P.VERMELHO \\
\end{tabular} & $\mathrm{PV}-7$ & 54 & 25 & $\bar{A}$ & $31 / 07 / 00$ & RB72-454 & $40 . \mathrm{C}$ & 1,9 & 14 & 144 & 67 & & 0,32 & 0,47 & 1,00 & 0,84 & 0,00 & 11,71 & 20,28 & 88,08 & 80,06 \\
\hline P.VERMELHO & PV-8 & 54 & 25 & B & $31 / 07 / 00$ & RB72-454 & $40 . \mathrm{C}$ & 1,3 & 16 & 114 & \begin{tabular}{|l|}
60 \\
\end{tabular} & & 0,22 & $\mid 0,53$ & 0,89 & 0,70 & 0,00 & 8,57 & & & \\
\hline S.J.F.ATHI & & 54 & 24 & A & \begin{tabular}{|l|}
$31 / 07 / 00$ \\
\end{tabular} & RB72-454 & $40 . \mathrm{C}$ & 1,9 & 14 & 144 & 67 & & 0,32 & 0,47 & 1,00 & 0,84 & 0,00 & 11,71 & 20,28 & 88,08 & 97,62 \\
\hline S.J.F.ATHI & & 54 & 24 & B & $31 / 07 / 00$ & RB72-454 & $40 . \mathrm{C}$ & 1,3 & 16 & 114 & 60 & & 0,22 & 0,53 & 0,89 & 0,70 & 0,00 & 8,57 & & & \\
\hline SAO PEDRO & STA B & 1 & 4 & A & $02 / 08 / 00$ & RB72-454 & $90 . \mathrm{C}$ & 0,8 & 5 & \begin{tabular}{|l|}
62 \\
\end{tabular} & 32 & & 0,13 & 0,21 & \begin{tabular}{|l|}
0,87 \\
\end{tabular} & 0,23 & 0,00 & 3,27 & 7,97 & 77,66 & 84,41 \\
\hline SAO PEDRO & STAB & 1 & 4 & B & $02 / 08 / 00$ & RB72-454 & $90 . \mathrm{C}$ & 0,3 & 3 & \begin{tabular}{|l|}
54 \\
\end{tabular} & 37 & & 0,05 & 0,13 & 0,79 & 0,95 & 0,00 & 4,70 & & & \\
\hline SAO PEDRO & $\begin{array}{l}\text { STA B } \\
\end{array}$ & 1 & 5 & A & $02 / 08 / 00$ & RB72-454 & $90 . \mathrm{C}$ & 0,8 & 8 & \begin{tabular}{|l|}
63 \\
\end{tabular} & 60 & & 0,13 & 0,31 & 0,88 & 0,70 & 0,00 & 5,01 & 6,65 & 76,54 & 68,5 \\
\hline SAO PEDRO & STAB & 1 & 5 & $\bar{B}$ & $02 / 08 / 00$ & RB72-454 & $90 . \mathrm{C}$ & 0,3 & 4 & 51 & 56 & & 0,05 & 0,17 & 0,76 & 0,62 & \begin{tabular}{|l|}
0,00 \\
\end{tabular} & 1,64 & & & \\
\hline PORT FELIZ & STAB & 2 & 15 & A & \begin{tabular}{|l|}
$11 / 09 / 00$ \\
\end{tabular} & RB72-454 & $80 . \mathrm{C}$ & 0,5 & 3 & \begin{tabular}{|l|}
63 \\
\end{tabular} & 33 & & 0,08 & 0,13 & 0,88 & 0,23 & 0,00 & 1,79 & 6,36 & 76,30 & \begin{tabular}{ll|}
66,81 \\
\end{tabular} \\
\hline PORT FELIZ & STAB & 2 & 15 & B & \begin{tabular}{|l|}
$11 / 09 / 00$ \\
\end{tabular} & RB72-454 & $80 . \mathrm{C}$ & 0,6 & 8 & 60 & 35 & & 0,10 & 0,31 & 0,85 & 0,92 & 0,00 & 4,57 & & & \\
\hline SANTA FE & STAB & 2 & 27 & A & \begin{tabular}{|l|}
$11 / 09 / 00$ \\
\end{tabular} & 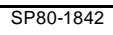 & $30 . \mathrm{C}$ & 0,6 & 8 & \begin{tabular}{|l|}
60 \\
\end{tabular} & 30 & & 0,10 & 0,31 & 0,85 & 0,21 & 0,00 & 7,26 & 14,03 & 82,79 & 50,47 \\
\hline SANTA FE & STAB & 2 & 27 & B & $11 / 09 / 00$ & SP80-1842 & $30 . \mathrm{C}$ & 0,3 & 3 & 48 & 41 & & 0,05 & 0,13 & 0,24 & 1,02 & 0,00 & 6,77 & & & \\
\hline \begin{tabular}{|l|} 
AREIA BRAN \\
\end{tabular} & STAB & 7 & 80 & $\bar{A}$ & \begin{tabular}{|l|}
$13 / 09 / 00$ \\
\end{tabular} & $\begin{array}{l}\text { RB83-5089 } \\
\end{array}$ & $70 . \mathrm{C}$ & 0,5 & 4 & \begin{tabular}{|l|}
69 \\
\end{tabular} & 24 & & 0,08 & $\mid 0,17$ & 0,94 & 0,17 & 0 & 4,98 & 7,22 & 77,03 & 70,17 \\
\hline \begin{tabular}{|l|} 
AREIA BRAN \\
\end{tabular} & STAB & 7 & 80 & B & \begin{tabular}{|l|}
$13 / 09 / 00$ \\
\end{tabular} & RB83-5089 & $70 . \mathrm{C}$ & 0,5 & 1 & 58 & 27 & & 0,08 & $\mid 0,04$ & 0,83 & 0,19 & 0,0 & 2,24 & & & \\
\hline \begin{tabular}{|l|} 
AREIA BRAN \\
\end{tabular} & STAB & 7 & 81 & A & \begin{tabular}{|l|}
$13 / 09 / 00$ \\
\end{tabular} & RB83-5486 & $60 . \mathrm{C}$ & 0,6 & 5 & 100 & 12 & & 0,10 & 0,21 & 0,75 & 0,09 & 0,00 & 5,31 & 10,30 & 79,63 & 78,4 \\
\hline AREIA BRAN & STAB & 7 & 81 & B & \begin{tabular}{|l|}
$13 / 09 / 00$ \\
\end{tabular} & RB83-5486 & $60 . \mathrm{C}$ & 0,5 & 4 & \begin{tabular}{|l|}
94 \\
\end{tabular} & 14 & & 0,08 & $\mid 0,17$ & 0,69 & 0,10 & 0,00 & 4,99 & & & \\
\hline \begin{tabular}{|l|} 
AREIA BRAN \\
\end{tabular} & STAB & 7 & 82 & $\bar{A}$ & \begin{tabular}{|l|}
$13 / 09 / 00$ \\
\end{tabular} & RB72-454 & $70 . \mathrm{C}$ & 0,5 & 13 & \begin{tabular}{|l|}
69 \\
\end{tabular} & 39 & & 0,08 & 0,44 & 0,94 & 0,98 & 0,00 & 6,61 & 13,23 & 82,11 & 69,10 \\
\hline AREIA BRAN & STAB & 7 & 82 & B & $13 / 09 / 00$ & RB72-454 & $70 . \mathrm{C}$ & 0,5 & 18 & 59 & \begin{tabular}{|l|}
42 \\
\end{tabular} & & 0,08 & 0,58 & 0,84 & 1,03 & 0,00 & 6,62 & & & \\
\hline \begin{tabular}{|l|} 
AREIA BRAN \\
\end{tabular} & STAB & 7 & 83 & A & \begin{tabular}{|l|}
$13 / 09 / 00$ \\
\end{tabular} & RB72-454 & $70 . \mathrm{C}$ & 0,5 & 4 & 78 & 25 & & 0,08 & $\mid 0,17$ & 0,53 & 0,18 & 0,00 & 3,77 & 6,90 & 76,76 & 56,2 \\
\hline \begin{tabular}{|l|} 
AREIA BRAN \\
\end{tabular} & STAB & 7 & 83 & $\bar{B}$ & \begin{tabular}{|l|}
$13 / 09 / 00$ \\
\end{tabular} & RB72-454 & $70 . \mathrm{C}$ & 0,3 & 1 & 70 & 32 & & 0,05 & 0,04 & 0,95 & 0,23 & \begin{tabular}{|l|}
0,00 \\
\end{tabular} & 3,13 & & & \\
\hline \begin{tabular}{|l|} 
AREIA BRAN \\
\end{tabular} & STAB & 7 & 88 & A & $13 / 09 / 00$ & $\begin{array}{l}\text { RB83-5089 } \\
\end{array}$ & $70 . \mathrm{C}$ & 0,5 & 4 & 84 & \begin{tabular}{|l|}
23 \\
\end{tabular} & & 0,08 & 0,17 & 0,59 & 0,16 & 0,00 & 3,91 & 5,61 & 75,66 & 85,5 \\
\hline \begin{tabular}{|l|} 
AREIA BRAN \\
\end{tabular} & STAB & 7 & 88 & B & \begin{tabular}{|l|}
$13 / 09 / 00$ \\
\end{tabular} & $\begin{array}{l}\text { RB83-5089 } \\
\end{array}$ & $70 . \mathrm{C}$ & 0,3 & 3 & 76 & 15 & & 0,05 & 0,13 & 0,51 & 0,11 & 0,00 & 1,70 & & & \\
\hline \begin{tabular}{|l|} 
PITANGA \\
\end{tabular} & $L 13+L 12$ & 15 & 59 & A & \begin{tabular}{|l|}
$13 / 09 / 00$ \\
\end{tabular} & SP80-1816 & $30 . \mathrm{C}$ & 0,5 & 56 & 42 & 19 & 29 & 0,08 & 1,00 & 0,21 & 0,13 & 0,58 & \begin{tabular}{l|l}
8,27 \\
\end{tabular} & 16,80 & 85,13 & 67,43 \\
\hline PITANGA & $L 13+L 12$ & 15 & 59 & B & $13 / 09 / 00$ & SP80-1816 & $30 . \mathrm{C}$ & 0,5 & 71 & 43 & 20 & \begin{tabular}{|l|}
35 \\
\end{tabular} & 0,08 & 1,00 & 0,22 & 0,14 & 0,70 & 8,52 & & & \\
\hline \begin{tabular}{|l|} 
PITANGA \\
\end{tabular} & $\mathrm{LI} 3+\mathrm{LI} 2$ & 15 & 60 & A & \begin{tabular}{|l|}
$13 / 09 / 00$ \\
\end{tabular} & SP80-1816 & $30 . \mathrm{C}$ & 0,5 & 24 & \begin{tabular}{|l|}
44 \\
\end{tabular} & \begin{tabular}{|l|}
22 \\
\end{tabular} & \begin{tabular}{|l|}
29 \\
\end{tabular} & 0,08 & 0,73 & 0,22 & 0,16 & 0,58 & 7,62 & 13,85 & 82,63 & 52,95 \\
\hline PITANGA & $\mathrm{LI} 3+\mathrm{LI} 2$ & 15 & 60 & B & $13 / 09 / 00$ & SP80-1816 & $30 . \mathrm{C}$ & 0,3 & 6 & 41 & 24 & \begin{tabular}{|l|}
35 \\
\end{tabular} & 0,05 & 0,25 & 0,21 & 0,17 & 0,70 & 6,23 & & & \\
\hline \begin{tabular}{|l|} 
AREIA BRAN \\
\end{tabular} & STAB & 1 & 1 & A & 04/10/00 & $\begin{array}{l}\text { RB80-6043 } \\
\end{array}$ & $70 . \mathrm{C}$ & 1,2 & 4 & \begin{tabular}{|l|}
44 \\
\end{tabular} & \begin{tabular}{|l|}
22 \\
\end{tabular} & & 0,20 & 0,17 & 0,22 & 0,16 & 0,00 & 0,95 & 1,08 & 71,83 & 67,3 \\
\hline AREIA BRAN & STAB & 1 & 1 & B & 04/10/00 & RB80-6043 & $70 . \mathrm{C}$ & 0,6 & 1 & 48 & 12 & & 0,10 & 0,04 & 0,24 & 0,09 & 0,00 & 0,13 & & & \\
\hline AREIA BRAN & STAB & 1 & 2 & $\bar{A}$ & $04 / 10 / 00$ & RB80-6043 & $70 . \mathrm{C}$ & 1,5 & 4 & 58 & $\mid 18$ & & 0,25 & 0,17 & 0,83 & 0,13 & 0,00 & 3,15 & 5,44 & 75,52 & 59,0 \\
\hline AREIA BRAN & STAB & 1 & 2 & B & $04 / 10 / 00$ & RB80-6043 & $70 . \mathrm{C}$ & 0,8 & 1 & \begin{tabular}{|l|}
69 \\
\end{tabular} & \begin{tabular}{|l|}
7 \\
\end{tabular} & & 0,13 & 0,04 & \begin{tabular}{|l|}
0,94 \\
\end{tabular} & 0,05 & 0,00 & 2,29 & & & \\
\hline SAO LUIS & PV9 & \begin{tabular}{|l|l|}
15015 \\
\end{tabular} & 161 & A & 04/10/00 & $\begin{array}{l}\text { RB83-5486 } \\
\end{array}$ & $70 . \mathrm{C}$ & 0,6 & 5 & \begin{tabular}{|l|}
56 \\
\end{tabular} & \begin{tabular}{|l|}
24 \\
\end{tabular} & \begin{tabular}{|l|}
21 \\
\end{tabular} & 0,10 & 0,21 & 0,81 & 0,17 & 0,42 & 4,23 & 6,97 & 76,8 & 71,50 \\
\hline SAO LUIS & $\overline{P V S}$ & 15015 & 161 & $\bar{B}$ & 04/10/00 & 86 & $70 . \mathrm{C}$ & 0,5 & 1 & 46 & 17 & \begin{tabular}{|l|}
43 \\
\end{tabular} & 0,08 & 0,04 & 0,23 & 0,12 & \begin{tabular}{|l|}
0,86 \\
\end{tabular} & 2,73 & & & \\
\hline SAO LUIS & PV9 & \begin{tabular}{|l|l|}
15015 \\
\end{tabular} & \begin{tabular}{|l|}
170 \\
\end{tabular} & A & 04/10/00 & $\begin{array}{l}\text { RB83-5089 } \\
\end{array}$ & $70 . \mathrm{C}$ & 1,0 & 5 & \begin{tabular}{|l|}
60 \\
\end{tabular} & 30 & \begin{tabular}{|l|}
21 \\
\end{tabular} & 0,17 & 0,21 & 0,85 & 0,21 & \begin{tabular}{|l|}
0,42 \\
\end{tabular} & 6,48 & 11,98 & 81,05 & 58,15 \\
\hline SAOLUIS & PV9 & \begin{tabular}{|l|l|}
15015 \\
\end{tabular} & 170 & $\bar{B}$ & 04/10/00 & RB83-5089 & $70 . \mathrm{C}$ & 0,6 & 3 & \begin{tabular}{|l|}
58 \\
\end{tabular} & 27 & \begin{tabular}{|l|}
43 \\
\end{tabular} & 0,10 & 0,13 & \begin{tabular}{|l|}
0,83 \\
\end{tabular} & 0,19 & 0,86 & 5,50 & & & \\
\hline S.A.PERRON & PV9 & 34 & 1 & $\bar{A}$ & $20 / 10 / 00$ & RB78-5148 & $60 . \mathrm{C}$ & 2,8 & 6 & 56 & 39 & 21 & 0,47 & 0,25 & 0,81 & 0,98 & 0,42 & 8,50 & 17,67 & 85,87 & 67,28 \\
\hline \begin{tabular}{|l|} 
S.A.PERRON \\
\end{tabular} & PV9 & 34 & 1 & $\bar{B}$ & \begin{tabular}{|l|}
$20 / 10 / 00$ \\
\end{tabular} & $\begin{array}{l}\text { RB78-5148 } \\
\end{array}$ & $60 . \mathrm{C}$ & 2,0 & 4 & \begin{tabular}{|l|}
52 \\
\end{tabular} & 46 & \begin{tabular}{|l|}
43 \\
\end{tabular} & 0,33 & 0,17 & 0,77 & 1,10 & 0,86 & 9,17 & & & \\
\hline STA.JUL.II & PV7 & 41 & 6 & $A$ & 20 & SP70-1143 & $80 . \mathrm{C}$ & 1,0 & 6 & 42 & 19 & & 0,17 & 0,25 & 0,21 & 0,13 & 0,00 & 0,11 & $-0,52$ & 70,48 & 46,02 \\
\hline STA.JUL.II & $\mathrm{PV7}$ & 41 & 6 & B & \begin{tabular}{|l|}
$20 / 10 / 00$ \\
\end{tabular} & SP70-1143 & $80 . \mathrm{C}$ & $\overline{0,5}$ & 3 & 45 & 15 & & $\overline{0,08}$ & 0,13 & 0,23 & 0,11 & $\mid 0,00$ & $-0,63$ & & & \\
\hline STA.JUL.II & PV7 & 41 & 7 & A & 20/10/00 & RB72-454 & $70 . \mathrm{C}$ & 1,0 & 6 & 58 & 46 & & 0,17 & 0,25 & 0,83 & 1,10 & 0,00 & 6,29 & 13,31 & 82,18 & 85,58 \\
\hline STA.JUL.II & PV7 & 41 & 7 & B & 20/10/00 & RB72-454 & $70 . \mathrm{C}$ & 0,5 & 23 & 57 & 45 & & 0,08 & 0,70 & 0,82 & 1,08 & 0,00 & 7,01 & & & \\
\hline \begin{tabular}{|l|} 
STA.JUL.II \\
\end{tabular} & $\mathrm{PV}$ & 41 & 8 & A & \begin{tabular}{|l|}
$20 / 10 / 00$ \\
\end{tabular} & RB72-454 & $70 . \mathrm{C}$ & 0,8 & 4 & 62 & 32 & & 0,13 & 0,17 & 0,87 & 0,23 & 0 & 3,44 & 8,56 & 78,16 & 71,91 \\
\hline STA.JUL.II & PV7 & 41 & 8 & B & \begin{tabular}{|l|}
$20 / 10 / 00$ \\
\end{tabular} & RB72-454 & $70 . \mathrm{C}$ & 0,5 & 3 & 53 & 47 & & 0,08 & 0,13 & 0,78 & 1,12 & 0,00 & 5,12 & & & \\
\hline STA.JUL.II & PV7 & 41 & 9 & A & $20 / 10 / 00$ & SP70-1143 & $80 . \mathrm{C}$ & 0,6 & 3 & 47 & 10 & & 0,10 & 0,13 & 0,24 & $\mid 0,07$ & 0,00 & $-0,43$ & 0,32 & 71,19 & 56,86 \\
\hline ..II & $\mathrm{PV}$ & 41 & 9 & B & 20/10/00 & SP7 & $80 . \mathrm{C}$ & 0,5 & 1 & 57 & 8 & & 0,08 & 0,04 & 0,82 & 0,06 & 0,00 & 0,76 & & & \\
\hline STA.JUL.II & $\overline{P V}$ & 41 & 10 & $\bar{A}$ & 0 & RB72-454 & $60 . \mathrm{C}$ & 2,1 & 9 & 48 & 12 & & 0,35 & 0,33 & 0,24 & 0,09 & 0,00 & 2,80 & 4,35 & 74,60 & 88,18 \\
\hline STA.JUL.II & PV7 & 41 & 10 & B & 20/10/00 & RB72-454 & $60 . \mathrm{C}$ & 0,6 & 4 & 48 & 12 & & 0,10 & 0,17 & 0,24 & $\mid 0,09$ & 0,00 & 1,55 & & & \\
\hline \begin{tabular}{|l|} 
STA.JUL.II \\
\end{tabular} & PV7 & 41 & 12 & A & \begin{tabular}{|l|}
$20 / 10 / 00$ \\
\end{tabular} & RB72-454 & $60 . \mathrm{C}$ & 1,3 & 4 & 50 & 32 & & 0,22 & 0,17 & 0,75 & 0,23 & \begin{tabular}{|l|}
0,00 \\
\end{tabular} & 4,08 & 10,73 & 80,00 & 53,75 \\
\hline \begin{tabular}{|l|} 
STA.JUL.II \\
\end{tabular} & PV7 & 41 & 12 & B & \begin{tabular}{|l|}
$20 / 10 / 00$ \\
\end{tabular} & RB72-454 & $60 . \mathrm{C}$ & 0,5 & 10 & 58 & 41 & & 0,08 & 0,36 & 0,83 & 1,02 & 0 & 6,65 & & & \\
\hline STA.JUL.II & PV7 & 41 & 13 & $\bar{A}$ & \begin{tabular}{|l|}
$20 / 10 / 00$ \\
\end{tabular} & RB72-454 & $60 . \mathrm{C}$ & 0,8 & 4 & 47 & 10 & & 0,13 & 0,17 & 0,24 & 0,07 & 0,00 & 1,37 & 2,65 & 73,16 & $\overline{78,38}$ \\
\hline STA.JUL.II & PV & 41 & 13 & B & $0 / 00$ & RB72-454 & $60 . \mathrm{C}$ & 0,5 & 3 & 47 & 19 & & 0,08 & 0,13 & 0,24 & 0,13 & 0,00 & 1,29 & & & \\
\hline \begin{tabular}{|l|} 
STA.JUL.II \\
\end{tabular} & PV7 & 41 & 15 & $\mathrm{~A}$ & \begin{tabular}{|l|}
$20 / 10 / 00$ \\
\end{tabular} & RB72-454 & $70 . \mathrm{C}$ & 0,6 & 4 & 49 & 14 & & 0,10 & 0,17 & 0,25 & 0,10 & 0,00 & 0,93 & 2,79 & 73,28 & 77,04 \\
\hline \begin{tabular}{|l} 
STA.JUL.II \\
\end{tabular} & PV7 & 41 & 15 & B & \begin{tabular}{|l|}
$20 / 10 / 00$ \\
\end{tabular} & RB72-454 & $70 . \mathrm{C}$ & 0,5 & 3 & 51 & 7 & & 0,08 & 0,13 & 0,76 & 0,05 & 0,00 & 1,85 & & & \\
\hline \begin{tabular}{|l|} 
STA.JUL.II \\
\end{tabular} & PV7 & 41 & 16 & A & $20 / 10 / 00$ & RB72-454 & $70 . \mathrm{C}$ & 0,6 & 4 & 48 & 12 & & 0,10 & 0,17 & 0,24 & 0,09 & 0,00 & 0,73 & 2,18 & 72,76 & 58,89 \\
\hline TA.JUL.II & PV7 & 41 & 16 & B & 20/10/00 & RB72-454 & 70.C & 0,3 & 1 & 50 & 6 & & 0,05 & 0,04 & 0,75 & 0,04 & 0,00 & 1,45 & & & \\
\hline VISTA SH & & 6 & 100 & A & 24/10/00 & RB72-454 & $60 . \mathrm{C}$ & 1,0 & 3 & 81 & 69 & & 0,17 & 0,13 & 0,56 & 0,88 & 0,00 & 4,97 & 10,20 & 79,55 & 45,8 \\
\hline
\end{tabular}


85

\begin{tabular}{|c|c|c|c|c|c|c|c|c|c|c|c|c|c|c|c|c|c|c|c|c|c|}
\hline nome & & & & & & & & teores & & & & & pontuação & & & & & & & & \\
\hline Fazenda & Solo & zona & talhão & horiz & coleta & Variedade & Estágio & $\bar{K}$ & $\mathrm{P}$ & \begin{tabular}{|l|l|}
$T$ & \\
\end{tabular} & \begin{tabular}{|l|l} 
\\
\end{tabular} & $\operatorname{Arg}$ & $\mathrm{K}$ & $P$ & \begin{tabular}{|l|l}
$T$ \\
\end{tabular} & \begin{tabular}{|lll}
$\mathrm{v}$ & & \\
\end{tabular} & \begin{tabular}{|l|} 
Arg \\
\end{tabular} & \begin{tabular}{|l|}
$\mathrm{NP}$ \\
\end{tabular} & \begin{tabular}{|l|} 
Ps \\
\end{tabular} & \begin{tabular}{|l|} 
prod estima \\
\end{tabular} & \begin{tabular}{|l|} 
prod medida \\
\end{tabular} \\
\hline B.VISTA SH & & 6 & 100 & $\bar{B}$ & 24/10/00 & RB72-454 & $60 . \mathrm{C}$ & 0,8 & 3 & 93 & \begin{tabular}{|c|}
69 \\
\end{tabular} & & $\overline{0,13}$ & 0,13 & 0,68 & 0,88 & 0,00 & \begin{tabular}{|l|}
5,23 \\
\end{tabular} & & & \\
\hline B.VISTA SH & & 6 & 104 & A & 24/10/00 & RB72-454 & $60 . \mathrm{C}$ & 2,1 & 5 & 156 & 78 & & 0,35 & 0,21 & 1,00 & 1,00 & 0,00 & \begin{tabular}{|l|}
7,98 \\
\end{tabular} & \begin{tabular}{|l|}
15,23 \\
\end{tabular} & 83,80 & 52,81 \\
\hline B.VISTA SH & & 6 & 104 & B & $24 / 10 / 00$ & RB72-454 & $60 . \mathrm{C}$ & 1,5 & 4 & 160 & 78 & & 0,25 & 0,17 & 1,00 & 1,00 & 0,00 & 7,25 & & & \\
\hline P.VERMELHO & PV-7 & 59 & 2 & A & \begin{tabular}{|l|}
$24 / 10 / 00$ \\
\end{tabular} & RB72-454 & $70 . \mathrm{C}$ & 1,0 & 6 & 44 & \begin{tabular}{|l|}
43 \\
\end{tabular} & & 0,17 & 0,25 & 0,22 & 1,05 & 0,00 & \begin{tabular}{|l|}
5,96 \\
\end{tabular} & 8,89 & 78,44 & 66,17 \\
\hline P.VERMELHO & PV-7 & 59 & 2 & B & $24 / 10 / 00$ & RB72-454 & $70 . \mathrm{C}$ & 0,6 & 3 & 38 & 42 & & 0,10 & 0,13 & 0,19 & 1,03 & 0,00 & \begin{tabular}{|l|}
2,92 \\
\end{tabular} & & & \\
\hline \begin{tabular}{|l|} 
P.VERMELHO \\
\end{tabular} & PV-7 & 59 & 3 & $\bar{A}$ & $24 / 10 / 00$ & RB72-454 & $70 . \mathrm{C}$ & 0,6 & 5 & 40 & \begin{tabular}{|l|}
22 \\
\end{tabular} & & 0,10 & 0,21 & 0,20 & 0,16 & 0,00 & 0,79 & \begin{tabular}{|l|}
1,76 \\
\end{tabular} & 72,41 & 70,08 \\
\hline P.VERMELHO & PV-7 & 59 & 3 & $\mathrm{~B}$ & $24 / 10 / 00$ & RB72-454 & $70 . \mathrm{C}$ & 0,6 & 10 & \begin{tabular}{|l|}
39 \\
\end{tabular} & 20 & & 0,10 & 0,36 & 0,20 & 0,14 & 0,00 & \begin{tabular}{|l|}
0,97 \\
\end{tabular} & & & \\
\hline \begin{tabular}{|l|} 
P.VERMELHO \\
\end{tabular} & PV-7 & 59 & 5 & A & \begin{tabular}{|l|}
$24 / 10 / 00$ \\
\end{tabular} & RB72-454 & $80 . C$ & 0,5 & 4 & 38 & 34 & & 0,08 & 0,17 & 0,19 & 0,24 & 0,00 & $-0,21$ & $\mid-1,08$ & 70,00 & 68,9 \\
\hline P.VERMELHO & PV-7 & 59 & 5 & B & $24 / 10 / 00$ & RB72-454 & $80 . \mathrm{C}$ & 0,5 & 3 & 37 & \begin{tabular}{|l|}
16 \\
\end{tabular} & & 0,08 & 0,13 & $\mid 0,19$ & 0,11 & \begin{tabular}{|l|}
0,00 \\
\end{tabular} & $-0,88$ & & & \\
\hline P.VERMELHO & $\mathrm{PV}-7$ & 59 & 6 & A & $24 / 10 / 00$ & RB72-454 & $70 . \mathrm{C}$ & 0,6 & 4 & 55 & \begin{tabular}{|l|}
38 \\
\end{tabular} & & 0,10 & 0,17 & 0,80 & \begin{tabular}{|l|}
0,97 \\
\end{tabular} & 0,00 & 5,13 & 5,36 & 75,45 & 66,59 \\
\hline P.VERMELHO & PV-7 & 59 & 6 & B & $24 / 10 / 00$ & RB72-454 & $70 . \mathrm{C}$ & 0,6 & 3 & 41 & \begin{tabular}{|l|}
17 \\
\end{tabular} & & 0,10 & 0,13 & 0,21 & 0,12 & 0,00 & \begin{tabular}{|l|}
0,23 \\
\end{tabular} & & & \\
\hline \begin{tabular}{|l|} 
P.VERMELHO \\
\end{tabular} & PV-7 & 59 & 8 & A & $24 / 10 / 00$ & RB72-454 & $70 . \mathrm{C}$ & 0,5 & 5 & 54 & \begin{tabular}{|l|}
29 \\
\end{tabular} & & 0,08 & 0,21 & 0,79 & 0,21 & 0,00 & 3,11 & 3,43 & 73,82 & 63,42 \\
\hline \begin{tabular}{|l|} 
P.VERMELLO \\
\end{tabular} & PV-7 & 59 & 8 & B & 24/10/00 & RB72-454 & $70 . \mathrm{C}$ & 0,3 & 4 & \begin{tabular}{|l|}
44 \\
\end{tabular} & \begin{tabular}{|l|}
13 \\
\end{tabular} & & 0,05 & 0,17 & 0,22 & 0,09 & 0,00 & \begin{tabular}{|l|}
0,31 \\
\end{tabular} & & & \\
\hline \begin{tabular}{|l|} 
P.VERMELLO \\
\end{tabular} & $P V-7$ & 59 & 9 & A & $24 / 10 / 00$ & RB72-454 & $70 . \mathrm{C}$ & 0,6 & 3 & 41 & \begin{tabular}{|l|}
24 \\
\end{tabular} & & 0,10 & 0,13 & 0,21 & 0,17 & 0,00 & 0,75 & 0,73 & 71,54 & 69,92 \\
\hline P.VERMELHO & PV-7 & 59 & 9 & $\mathrm{~B}$ & $24 / 10 / 00$ & RB72-454 & $70 . \mathrm{C}$ & 0,5 & 1 & 43 & 11 & & 0,08 & 0,04 & \begin{tabular}{|l|}
0,22 \\
\end{tabular} & 0,08 & 0,00 & $-0,02$ & & & \\
\hline P.VERMELHO & PV-7 & 59 & 10 & A & 24/10/00 & RB72-454 & $70 . \mathrm{C}$ & 0,6 & 4 & \begin{tabular}{|l|}
47 \\
\end{tabular} & \begin{tabular}{|l|}
27 \\
\end{tabular} & & 0,10 & 0,17 & 0,24 & 0,19 & 0,00 & \begin{tabular}{|l|}
1,11 \\
\end{tabular} & 1,20 & 71,93 & 62,61 \\
\hline P.VERMELHO & PV-7 & 59 & 10 & B & $24 / 10 / 00$ & RB72-454 & 70.C & 0,5 & 1 & 45 & 15 & & 0,08 & 0,04 & 0,23 & 0,11 & 0 & \begin{tabular}{|l|}
0,09 \\
\end{tabular} & & & \\
\hline B.VISTA SH & & 7 & 125 & A & $31 / 10 / 00$ & RB80-6043 & $80 . \mathrm{C}$ & 1,6 & 23 & 104 & \begin{tabular}{|l|}
61 \\
61
\end{tabular} & & 0,27 & 0,70 & 0,79 & 0,72 & 0,00 & 5,71 & \begin{tabular}{|l|}
9,31 \\
\end{tabular} & 78,80 & 68,3 \\
\hline B.VISTA SH & & 7 & \begin{tabular}{|l|}
125 \\
\end{tabular} & B & $31 / 10 / 00$ & RB80-6043 & $80 . \mathrm{C}$ & 1,3 & 6 & 106 & 55 & & 0,22 & 0,25 & 0,81 & 0,60 & 0,00 & \begin{tabular}{|l|}
3,61 \\
\end{tabular} & & & \\
\hline B.VISTA SH & & 7 & \begin{tabular}{|l|}
126 \\
\end{tabular} & A & $31 / 10 / 00$ & RB80-6043 & $80 . \mathrm{C}$ & 1,6 & 23 & 104 & 61 & & 0,27 & 0,70 & 0,79 & 0,72 & 0,00 & 5,71 & \begin{tabular}{|l|}
9,31 \\
\end{tabular} & 78,80 & 57,7 \\
\hline B.VISTA SH & & 7 & \begin{tabular}{|l|}
126 \\
\end{tabular} & B & $31 / 10 / 00$ & RB80-6043 & $80 . \mathrm{C}$ & 1,3 & 6 & 106 & \begin{tabular}{|l|}
55 \\
\end{tabular} & & 0,22 & 0,25 & $\mid 0,81$ & 0,60 & 0,00 & \begin{tabular}{|l|}
3,61 \\
\end{tabular} & & & \\
\hline B.VISTA SH & & 7 & 129 & A & $31 / 10 / 00$ & RB80-6043 & $80 . \mathrm{C}$ & 2,4 & 9 & 186 & 84 & & 0,40 & 0,33 & 1,00 & 1,00 & 0,00 & \begin{tabular}{|l|}
6,25 \\
\end{tabular} & 10,69 & 79,96 & 52,0 \\
\hline B.VISTA SH & & 7 & \begin{tabular}{|l|}
129 \\
\end{tabular} & B & \begin{tabular}{|l|}
$31 / 10 / 00$ \\
\end{tabular} & RB80-6043 & $80 . \mathrm{C}$ & 2,1 & 3 & 174 & \begin{tabular}{|l|}
64 \\
\end{tabular} & & 0,35 & 0,13 & 1,00 & 0,78 & 0,00 & \begin{tabular}{|l|}
4,44 \\
\end{tabular} & & & \\
\hline B.VISTA SH & & 7 & \begin{tabular}{|l|}
131 \\
\end{tabular} & A & $31 / 10 / 00$ & RB80-6043 & $80 . \mathrm{C}$ & 2,4 & 9 & 186 & \begin{tabular}{|l|}
84 \\
\end{tabular} & & 0,40 & 0,33 & 1,00 & 1,00 & 0,00 & \begin{tabular}{|l|}
6,25 \\
\end{tabular} & \begin{tabular}{|l|}
10,69 \\
\end{tabular} & 79,96 & 48,99 \\
\hline B.VISTA SH & & 7 & \begin{tabular}{|l|}
131 \\
\end{tabular} & B & $31 / 10 / 00$ & RB80-6043 & $80 . C$ & 2,1 & 3 & 174 & \begin{tabular}{|l|}
64 \\
\end{tabular} & & 0,35 & 0,13 & 1,00 & 0,78 & 0,00 & \begin{tabular}{|l|}
4,44 \\
\end{tabular} & & & \\
\hline B.VISTA SH & & 7 & \begin{tabular}{|l|}
134 \\
\end{tabular} & A & $31 / 10 / 00$ & $\begin{array}{l}\text { RB80-6043 } \\
\end{array}$ & $80 . \mathrm{C}$ & 1,3 & 8 & 114 & 74 & & 0,22 & 0,31 & 0,89 & $0,0,98$ & 0,00 & 5,38 & 10,01 & 79,39 & 54,21 \\
\hline B.VISTA SH & & 7 & 134 & B & $31 / 10 / 00$ & RB80-6043 & $80 . \mathrm{C}$ & 1,2 & 8 & 135 & \begin{tabular}{|l|}
62 \\
\end{tabular} & & 0,20 & 0,31 & 1,00 & 0,74 & 0,00 & 4,64 & & & \\
\hline B.VISTA SH & & 7 & 135 & A & $31 / 10 / 00$ & RB80-6043 & $80 . \mathrm{C}$ & 1,3 & 8 & 114 & 74 & & 0,22 & 0,31 & \begin{tabular}{|l|}
0,89 \\
\end{tabular} & 0,08 & 0 & 5,38 & 10,01 & 79,39 & 47,81 \\
\hline B.VISTA SH & & 7 & 135 & B & $31 / 10 / 00$ & RB80-6043 & $80 . \mathrm{C}$ & 1,2 & 8 & 135 & \begin{tabular}{|c|}
62 \\
\end{tabular} & & 0,20 & 0,31 & 1,00 & 0,74 & 0,00 & \begin{tabular}{|l|}
4,64 \\
\end{tabular} & & & \\
\hline B.VISTA SH & & 7 & \begin{tabular}{|l|}
138 \\
\end{tabular} & $\bar{A}$ & $31 / 10 / 00$ & $\begin{array}{l}\text { RB80-6043 } \\
\end{array}$ & $80 . \mathrm{C}$ & 1,3 & 8 & 114 & 74 & & $\overline{0,22}$ & 0,31 & 0,89 & 0,98 & 0,00 & 5,38 & 10,01 & 79,39 & 68,50 \\
\hline B.VISTA SH & & 7 & \begin{tabular}{|l|}
138 \\
\end{tabular} & B & $31 / 10 / 00$ & RB80-6043 & $80 . \mathrm{C}$ & 1,2 & 8 & 135 & \begin{tabular}{|c|}
62 \\
\end{tabular} & & 0,20 & 0,31 & 1,00 & 0,74 & 0,00 & \begin{tabular}{|l|}
4,64 \\
\end{tabular} & & & \\
\hline B.VISTA SH & & 7 & \begin{tabular}{|l|}
142 \\
\end{tabular} & A & $31 / 10 / 00$ & RB80-6043 & $80 . C$ & 3,6 & 14 & 158 & \begin{tabular}{|l|}
63 \\
\end{tabular} & & 0,60 & 0,47 & 1,00 & 0,76 & 0,00 & \begin{tabular}{|l|}
6,47 \\
\end{tabular} & \begin{tabular}{|l|}
9,06 \\
\end{tabular} & 78,58 & 46,98 \\
\hline B.VISTA SH & & 7 & \begin{tabular}{|l|}
142 \\
\end{tabular} & B & $31 / 10 / 00$ & $\begin{array}{l}\text { RB80-6043 } \\
\end{array}$ & $80 . \mathrm{C}$ & 2,6 & 2 & 256 & 31 & & 0,43 & 0,08 & 1,00 & 0,22 & 0,00 & \begin{tabular}{|l|}
2,59 \\
\end{tabular} & & & \\
\hline
\end{tabular}

